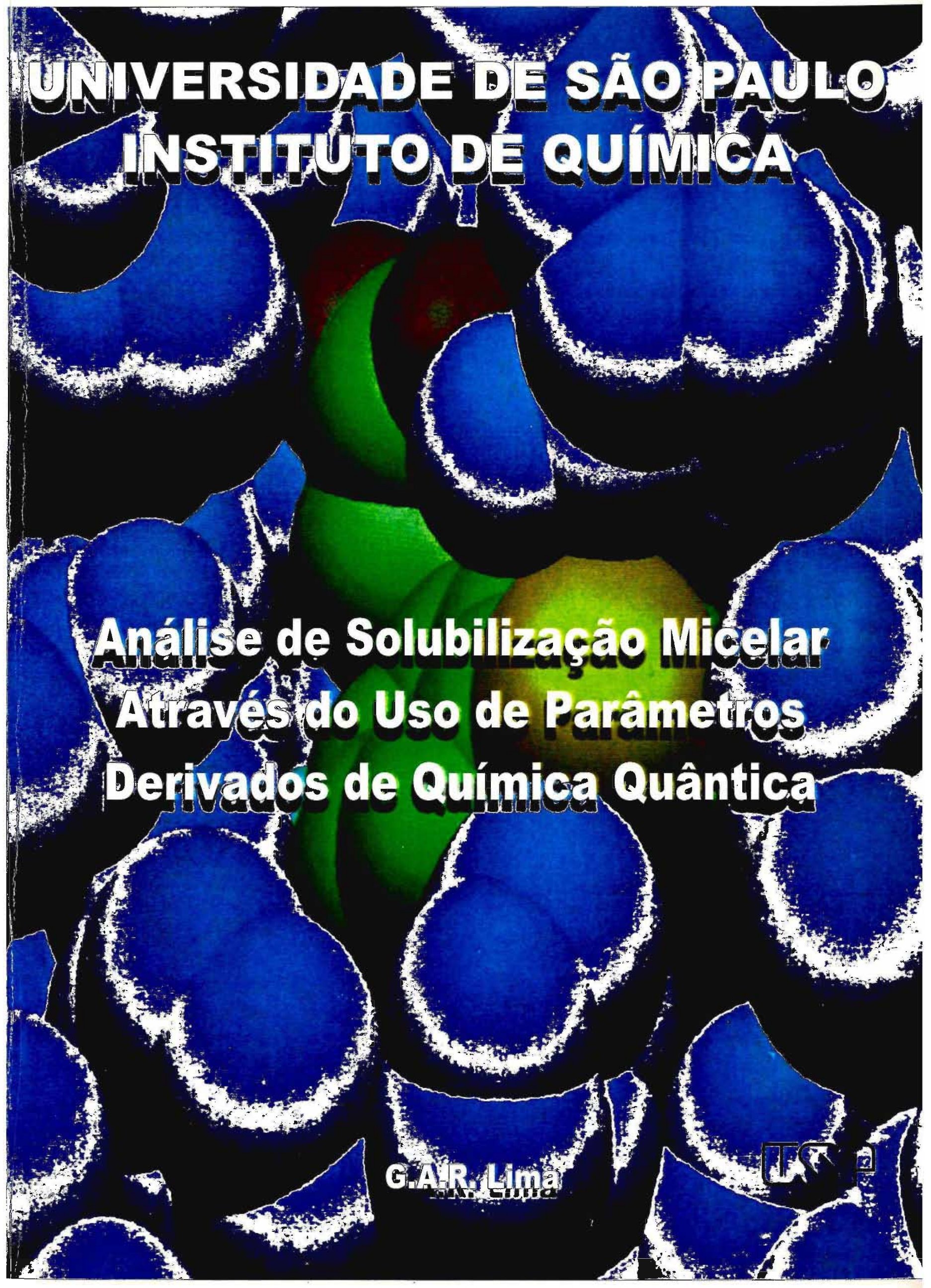


Universidade de São Paulo

Instituto de Química

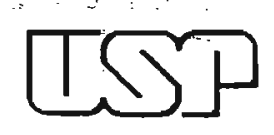

\title{
Análise de Solubilização Micelar Através do Uso de Parâmetros Derivados de Química Quântica
}

\author{
G. A. R. Lima
}

Tese de Doutorado

Orientador : Dr. Frank Herbert Quina

São Paulo

1999 
Ficha Catalográfica

Elaborada pela Divisão de Biblioteca e

Documentação do Conjunto das Químicas da USP.

\section{Lima, Gilson Alberto Rosa}

L732a Análise de solubilização micelar através do uso de parâmetros derivados de química quântica / Gilson Alberto Rosa Lima. -- São Paulo, 1999.

$124 \mathrm{p}$.

Tese (doutorado) - Instituto de Química da Universidade de São Paulo. Departamento de Química Fundamental.

Orientador: Quina, Frank Herbert

1. Físico-química : Orgânica 2. Química quântica 3. Detergentes: Físico-química 4. QSAR: Química farmacêutica I. T. II. Quina, Frank Herbert, orientador. 


\section{"Análise de Solubilização Micelar Através do Uso de Parâmetros Derivados de Química Quântica".}

\section{GILSON ALBERTO ROSA LIMA}

Tese de Doutorado submetida ao Instituto de Química da Universidade de São Paulo como parte dos requisitos necessários à obtenção do grau de Doutor em Ciências - Área: Físico-Química.

Aprovado por:

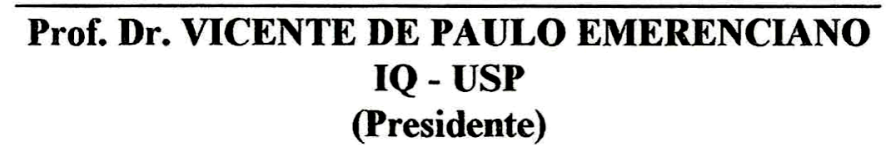

$\overline{\text { Prof. Dr. OMAR ABDEL MONEIN ABOU EL SEOUD }}$

IQ - USP

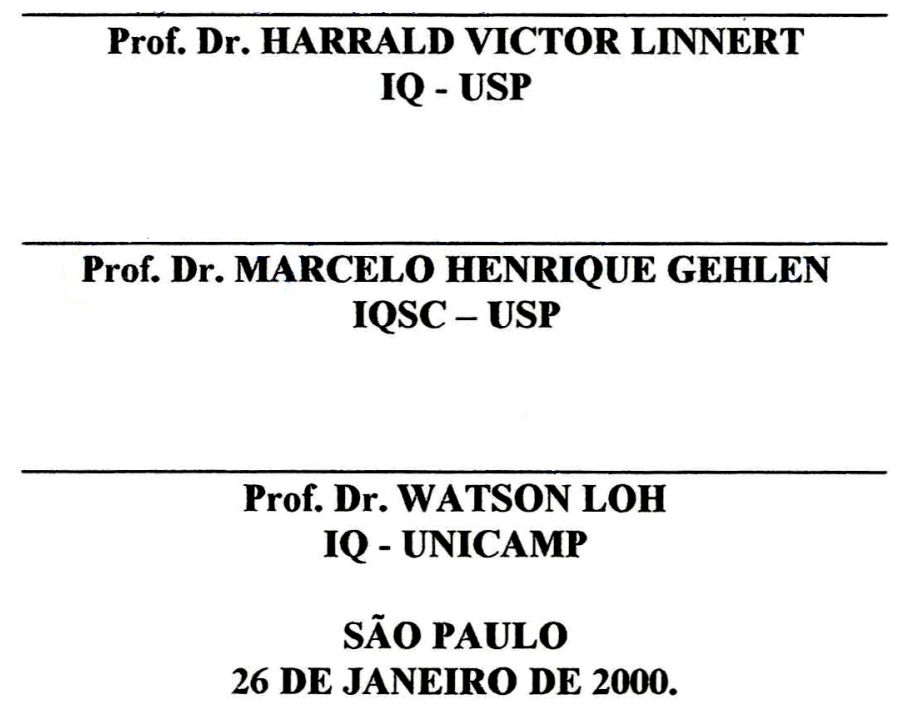




\section{Dedicação}

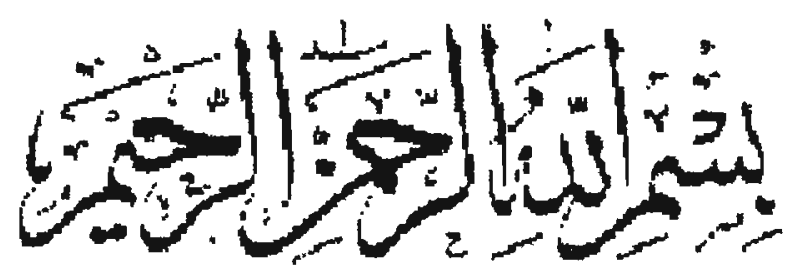

Em Nome de Deus, O Clemente, O Misericordioso.

Com Gemuína Humildade Eu Reconheço A Tua Ajuda, Oh Deus! No Verdadeiro Espirito Do Islam, Eu Aprecio A Tua Graça, Oh Deus! Agradeço A Ti De Todo O Meu Coração

Sem A Tua Orientação E Amor, Esse Trabalho Não Seria Possível Essa Humilde Contribuição Nunca Se Tornaria Realidade

E Se Valer A Pena Dedicar Este Trabalho Por Favor, Abençoe Com Tua Aceitação Este Trabalho Dedico, A Ti, Oh Meu Senhor!

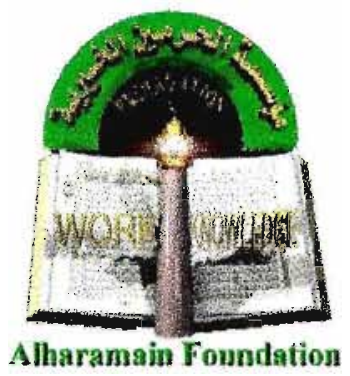




\section{Dedication}

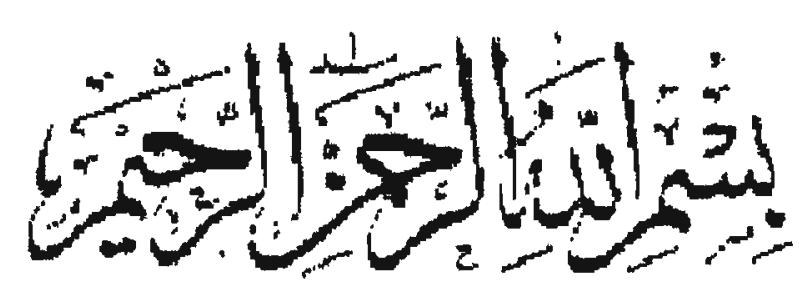

In The Name Of God The Most Gracious Most Merciful.

With Genuine Humility, I Acknowledge Your Aid, O God! In The True Spirit Of Islam, I Appreciate Your Grace, O God!

With All My Heart, I Thank You, O God I

Without Your Guidance And Love,

This Work Would Not Have Been Possible

Were It Not For Your Help and Cause,

This Humble Contribution Would Have Never Become A Reality. And If It Is Worth Dedicating,

Please Bless It With Your Acceptance;

It Is Dedicated To You, O God!

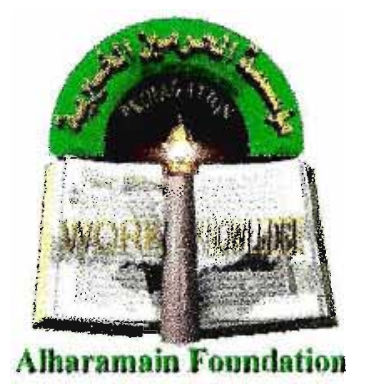




\section{Agradecimentos}

Agradeço aos meus pais Atalício S. Lima e Mariah H.R. Lima pelo constante apoio e pelos ensinamentos de vida. trabalho.

Agradeço ao Dr. F.H. Quina pela orientação e oportunidade de desenvolver este

Agradeço aos Drs. J.P.S. Farah e V.P. Emerenciano pelo incentivo e apoio e aos Drs. A. Fazzio e J.A. Kintop pela amizade e companheirismo.

Agradeço aos colegas do laboratório A.A. Freitas, C. Yihwa, C.M. Tcacenco, C.N. Ochiai, F.J.K. Tanabe, K. Shimizu, P. F.Moreira Jr. e a todas as pessoas que de alguma forma contribuíram para a realização deste trabalho.

Em especial agradeço às "Isabéis" pelo amor, carinho, compreensão, confiança e por tantos favores concedidos.

Este trabalho teve o suporte financeiro da Fundação de Amparo à Pesquisa do Estado de São Paulo - FAPESP (projeto temático 1994/3505-3) e PADCT-FINEP (Projeto No 6592-0063-00). 


\section{Índice}

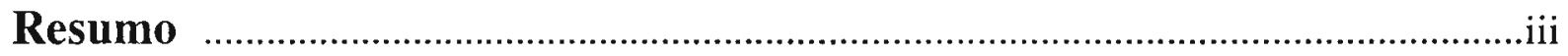

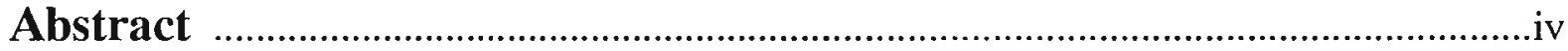

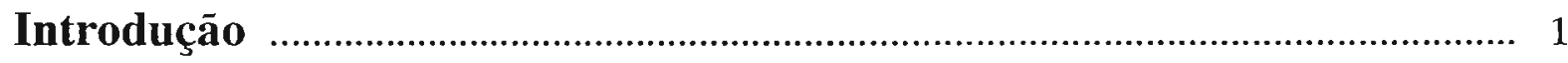

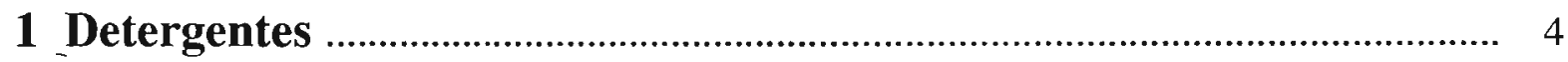

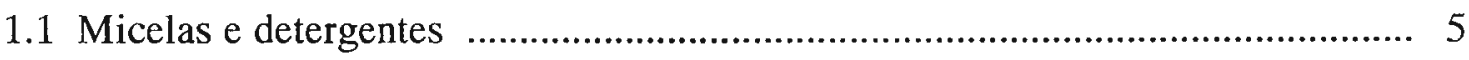

2 Correlação Linear de Energia Livre de Solvatação (LSERs) .............. 9

2.1 Correlação e regressão linear múltipla .................................................. 10

2.2 Correlação e causalidade .......................................................................... $\quad 10$

2.3 Relação linear de energia livre de solvatação …............................................ 11

2.4 Modelo de solvatação da cavidade ................................................................. 12

2.5 Aplicação da relação linear de energia livre de solvatação em sistemas
micelares

3 Correlação Linear Teórica de Energia Livre de Solvatação (TLSER) 20

3.1 Correlação linear teórica de energia livre de solvatação .............................. 21

3.2 Modelo de Famini ............................................................................... 21

4 Formalismo Matemático Hartree-Fock-Roothaan (semi-empírico)... 25

4.1 Cálculo de estrutura eletrônica …........................................................... 26

4.2 Teoria do orbital molecular ................................................................. 26

4.3 A equação de Schrödinger independente do tempo ................................. 27

4.4 Aproximação de Born-Oppenheimer ......................................................... 28

4.5 Aproximação de Hartree-Fock …................................................................ 30

4.6 Métodos SCF-LCAO semi-empíricos …................................................. 32

4.6.1 Aproximação dos elétrons de valência ................................................... 33

4.6.2 Aproximação ZDO-Zero Differential Overlap ........................................ 34

4.6.3 Elementos de matriz HFR .................................................................... 34

4.7 Método semi-empíricos MNDO-PM3 ................................................. 36

5 Metodologia: cálculo dos parâmetros teóricos ………………………..... 41

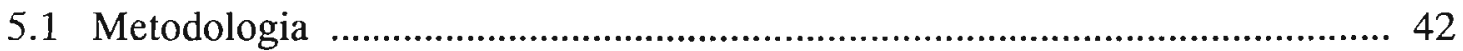

5.2 Volume do soluto ........................................................................... 42 
5.3 Interações por pontes de hidrogênio

5.3.1 $\left(q_{+}, \varepsilon_{\mathrm{l.ммо}}\right)$ caráter ácido das pontes de hidrogênio ................................. 43

$5.3 .2\left(|q-|, \varepsilon_{\text {помо }}\right)$ caráter básico das pontes de hidrogênio .............................. 43

5.3 .3 Cálculo da carga formal ....................................................................... 43

5.4 Momento de dipolo ......................................................................... 45

5.5 Dipolaridade / Polarizabilidade ............................................................. 46

5.5.1 Cálculo semi-empírico de polarizabilidade.............................................. 46

5.5.2 Procedimento MNDO-PM3 para cálculo da polarizabilidade ................ 47

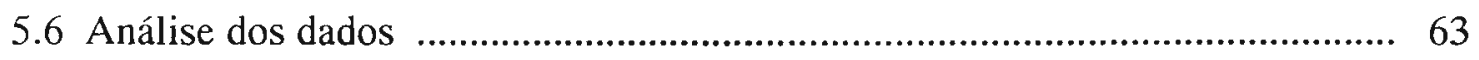

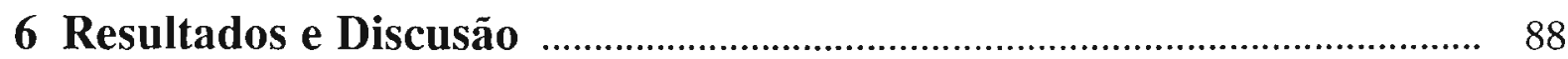

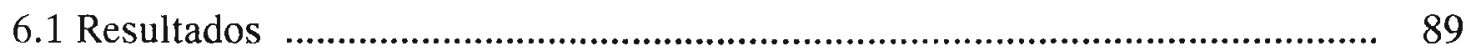

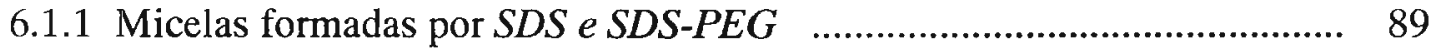

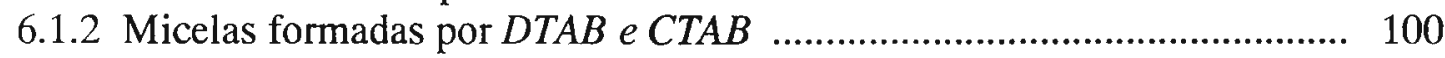

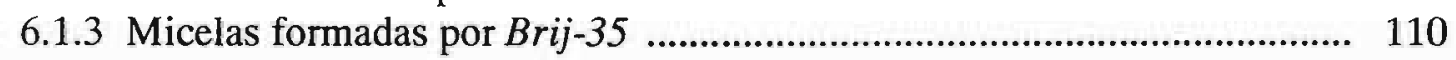

6.2 Limitações da metodologia ................................................................ 115

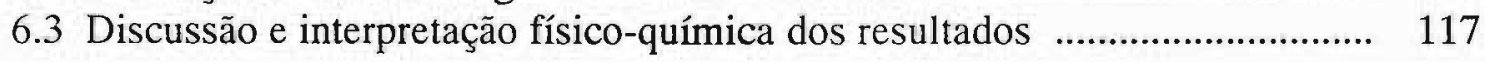

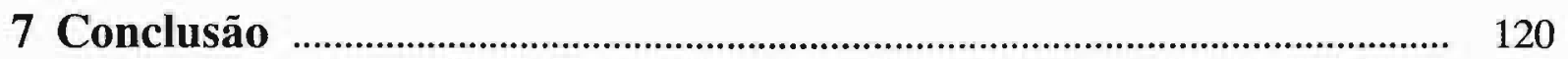

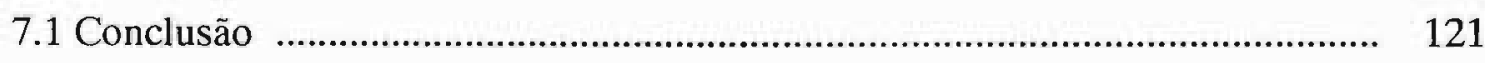

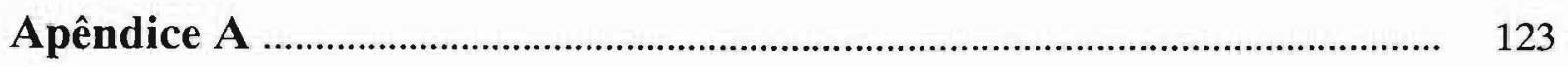

Trabalho Publicado no Journal of Molecular Structure (THEOCHEM) ............. 124 


\section{Resumo}

Uma das propriedades fundamentais de soluções de micelas aquosas é sua capacidade de solubililizar uma ampla faixa de solutos orgânicos com diferentes graus de polaridade e hidrofobicidade. Recentemente Quina et. al., estudaram as interações químicas específicas que governam a solubilidade para um ampla faixa de solutos orgânicos em micelas usando relações lineares de energia livre de solvatação ( $L S E R S$ ), baseado nos parâmetros empíricos do soluto desenvolvido por Abraham. O presente trabalho trata a solubilização de solutos orgânicos em micelas baseado em parâmetros obtidos unicamente da estrutura molecular do soluto através de cálculos de química quântica.

Nossos resultados mostram que valores experimentais de $K_{s}$ para solubilização de solutos orgânicos em detergentes aniônicos (micelas de $S D S$, dodecil sulfato de sódio e agregados de SDS com $P E G$-polietilenoglicol) podem ser reproduzidos usando parâmetros teóricos. A relação linear teórica de energia livre de solvatação resultante é quimicamente condizente com LSERs baseados em parâmetros empíricos do soluto.

No caso de detergentes catiônicos e não iônicos, a metodologia apresenta limitações, por que o método de cálculo de cargas formais usando a definição de análise populacional de Mulliken não descreve de maneira apropriada o caráter ácido e básico das pontes de hidrogênio entre os solutos e a água. Alternativas para resolver o problema da limitação imposta pelos parâmetros teóricos, mantendo a aproximação linear de energia livre, são discutidas. 


\begin{abstract}
One of the most fundamental properties of aqueous micelar solutions is their ability to solubilize a wide variety of organic solutes with quite distinct polarities and degree of hydrophobicity. Recently Quina et al., studied the specific chemical interactions governing the solubilities of a wide range of solutes in micelles using linear solvation energy relationships (LSERS) based on empirical solute parameters developed by Abraham. This work reports an investigation of the solubilization of organic solutes in micelles based on the use of theoretical solute parameters derived from quantum chemical calculations

Our results show that the experimental $K_{s}$ values for solubilization of organic solutes in anionic detergents (micelles of $S D S$-sodium dodecyl sulfate and aggregate formed between $S D S$ and $P E G$-poly(ethylene glycol)) can be reproduced using theoretical parameters. The resultant theoretical linear solvation energy relationships are chemically consistent with LSERs based on empirical solute parameters.

In the case of cationic and nonionic detergents, the theoretical parameters used to describe hydrogen bonding fail because methods of calculating formal charges using the concept of Mulliken populational analysis cannot describe hydrogen bonding donor and acceptor character in an appropriate way. Possible strategies to solve the theoretical descriptor problem in the context of the linear free energy approximation are discussed.
\end{abstract}




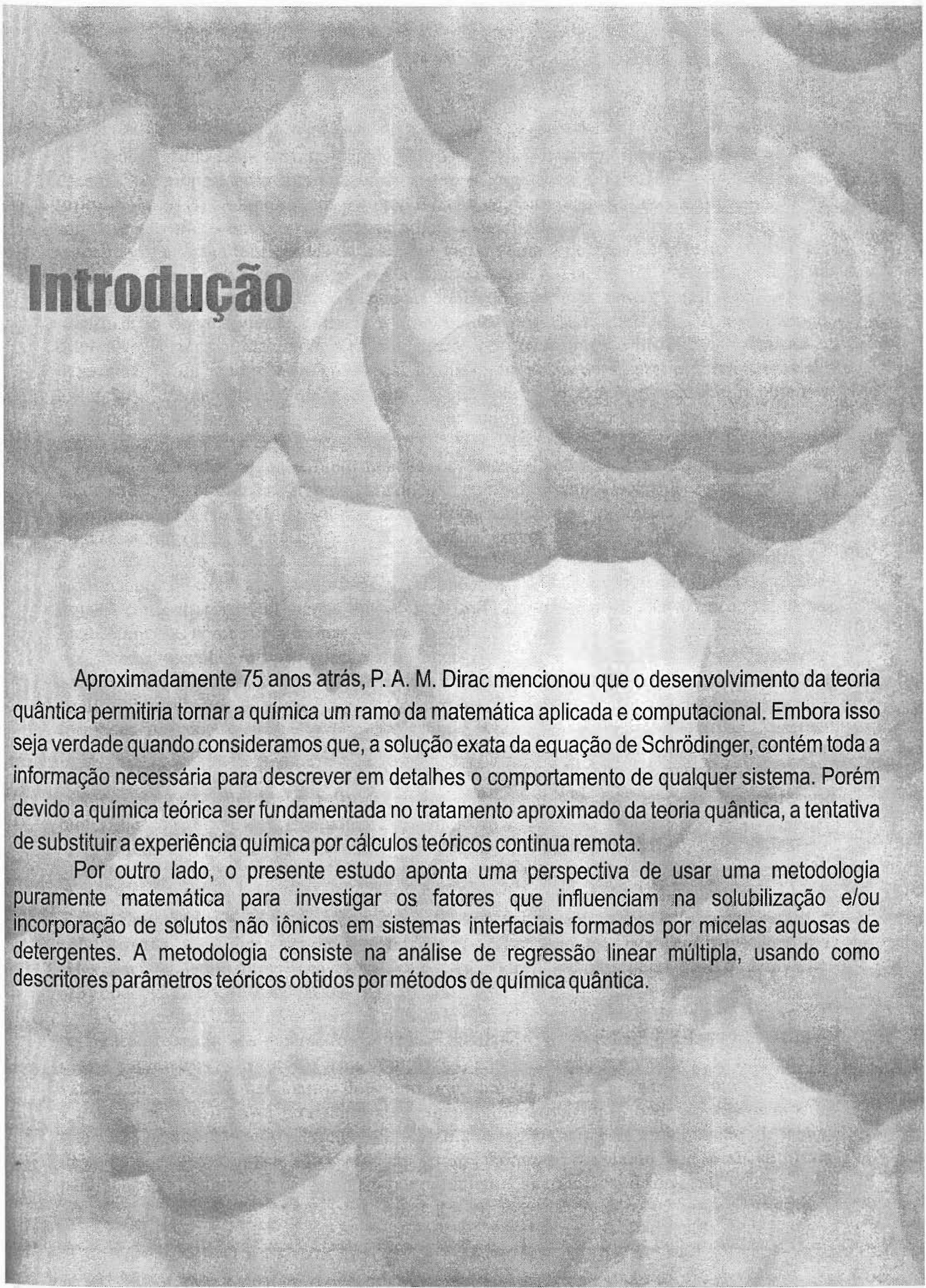




\section{Introdução}

Nosso grupo de pesquisa, liderado pelo Dr. F. H. Quina vem investigando, já há duas décadas, as propriedades interfaciais de sistemas organizados formados por detergentes (micelas aquosas) e/ou polímeros e a modificação de reatividade química e fotoquímica de sondas e substratos em presença dos mesmos. Estas investigações visam esclarecer: (a) a maneira em que as propriedades da interface variam com a densidade de carga elétrica ali presente e com a natureza dos íons presentes no sistema; (b) os fatores que influenciam na solubilização ou incorporação de pequenas moléculas ou íons nestas interfaces; e, (c) a modíficação de reatividade química e fotoquímica por estas interfaces. Aproveitam-se diferentes técnicas, incluindo técnicas fotofísicas (fluorimetria estática e dinâmica, determinação de rendimentos quânticos, fotólise por pulso de laser), espectroscopia de absorção e cromatografia líquida de alto desempenho. Em todos os estudos, o objetivo principal é o desenvolvimento de modelos físico-químicos capazes de reproduzir e prever fenômenos observados experimentalmente.

Uma das propriedades fundamentais de micelas aquosas é a sua capacidade de incorporar e solubilizar solutos orgânicos com diferentes polaridades e grau de hidrofobicidade. No caso de solutos não iônicos, costuma-se expressar essa propriedade através de um razão de concentração, $K_{s}$, definido ${ }^{1}$ como: $K_{s}=\left[S_{m i c}\right] /\left(\left[S_{a q}\right] C_{d}\right)$, onde $\left[S_{m i c}\right]$ é a concentração analítica do soluto ligado à fase organizada e $\left[S_{a q}\right]$, a concentração analítica do soluto no meio aquoso e $C_{d}$ a concentração do detergente micelizado, a qual é igual à concentração total menos a concentração crítica de micelização (concentração na qual as micelas começam a formar-se), isto é, $C_{d}=C_{t}-c m c$.

Recentemente, relaçōes lineares de energia livre de solvatação (LSERs - Linear Solvation Energy Relationships) vêm sendo utilizadas, com sucesso, para correlacionar e analisar coeficientes de partição para a incorporação de solutos não iônicos por micelas formadas por detergentes. No primeiro estudo, Quina et. al. ${ }^{2}$ empregaram correlações lineares de energia livre de solvatação baseado nos parâmetros do soluto propostos por Abraham ${ }^{3,4}$, mostrando que esta metodologia permite a previsão das eficácias de incorporação de solutos em micelas aquosas com base na interpretação da estrutura molecular do soluto e dos detergentes que compõem a micela.

Atualmente, outros grupos como Vitha et. al. ${ }^{\mathbf{5}, 6}$ e Abraham et. al. ${ }^{7}$, vêm aplicando essa metodologia ao estudo de solubilização em sistemas micelares. Mais recentemente, Quina et. al. demonstraram que esta metodologia também pode ser utilizada para correlacionar e prever tensões interfaciais entre líquidos orgânicos e a água e para analisar solubilização em micelas mistas formadas por brometo de hexadeciltrimetilamonia (Hexadecyltrimethylammonium bromide) e éter dodecíl polioxietileno (polyoxyethylene (23) dodecyl ether), (CTAB+BRIJ-35) ${ }^{9}$.

O principal fator que limita a utilidade de relações lineares de energia livre de solvatação baseado nos parâmetros de soluto tabulados por Abraham é a falta dos valores destes parâmetros para solutos mais complexos, uma limitação acentuada pelo fato que os valores de vários dos parâmetros do soluto precisam ser determinados experimentalmente. Por esse motivo, tem-se procurado abordagens alternativas para o desenvolvimento de relações lineares de energia livre de solvatação que sejam úteis para uma quantidade maior de solutos. A possibilidade sob estudo em nosso laboratório é a viabilidade de substituir os parâmetros experimentais de Abraham por parâmetros alternativos, de origem "teórica". 
Nesse estudo apresentamos pela primeira vez uma análise detalhada do uso de parâmetros calculados por métodos de química computacional, em relações lineares de energia livre para correlacionar com os valores experimentais de $K_{s}$.

Para esta finalidade, efetuamos cálculos semi-empíricos e ab-initio (usando o formalismo $H F$ ) para determinar os valores dos parâmetros teóricos, utilizando os programas MOPAC $-93^{10}$, AMSOL-5.4 $4^{11}$ e Gaussian-94 ${ }^{12}$.

\section{Referências}

1. Sepúlveda, L.; Lissi, E.; Quina, F. H.;1986, Adv. Colloid Interface Sci., 25, 1.

2. Quina, F. H.; Alonso, E. O.; Farah, J. P. S.; 1995, J. Phys. Chem., 99, 11708.

3. Abraham, M. H.; 1993, Chem. Soc. Rev., 22, 73.

4. Abraham, M. H.; Chadham, H. S.; Whiting, G. S.; Michell, R. C.; 1994, J. Pharm. Sci.; 83, 1085.

5. Vitha, M. F.; Weckwerth, J. D.; Odland, K.; Dema, V.; Carr, P. W.; 1996, J. Phys. Chem., 100, 18823.

6. Vitha, M. F.; Dallas, A. J.; Carr, P. W.; 1997, J. Colloid Interface Sci., 187, 179.

7. Abraham, M. H.; Chadha, H. S.; Dixon, J. P.; Rafols, C.; Treiner, C.;1997, J. Chem. Soc. Perkin Trans.2, 19.

8. Freitas, A.A.; Quina, F.H.; Carroll, F.A.;1997, J. Phys. Chem B;101, 7488.

9. Rodrigues, M. A.; Alonso, E. O.; Yihwa, C.; Farah, J. P. S.; Quina, F. H.;1999, Langmuir, 15, 6770.

10. Stewart, J. J. P.; Fujitsu Limited, Tokyo, Japan, 1993.

11. Hawkins, G. D.; Lynch, G. C.; Gissen, D. J.; Rossi, I.; Storer, J. W.; Liotard, D. A.; Cramer, C. J.; Truhlar, D. G.; QCPE, 1995.

12. Trucks, G. W.; Schlegel, H. B.; Gill, P. M. W.; Johnson, B. G.; Robb, M. A.; Cheesemam, J. R.; Keith, T.; Petersson, G. A.; Montgomery, J. A.; Raghavachari, K.; Al-Laham, M. A.; Zakrzewski, J.; Stefanov, B. B.; Nanayakkar, A. A.; Challacombe, M.; Peng, C. Y.; Ayala, P. Y.; Chen, W.; Wong, M. W.; Andrea, J. L.; Replogle, E. S.; Gomperts, R.; Martin, R. L.; Fox, D. J.; Binkley, J. S.; Defrees, D. J.; Baker, J.; Stewart, J. J. P.; Head-Gordon, M.; Gonzales, C. e Pople, J. A.; Gaussian-94, version C.3, Pittisburg PA, 1995. 


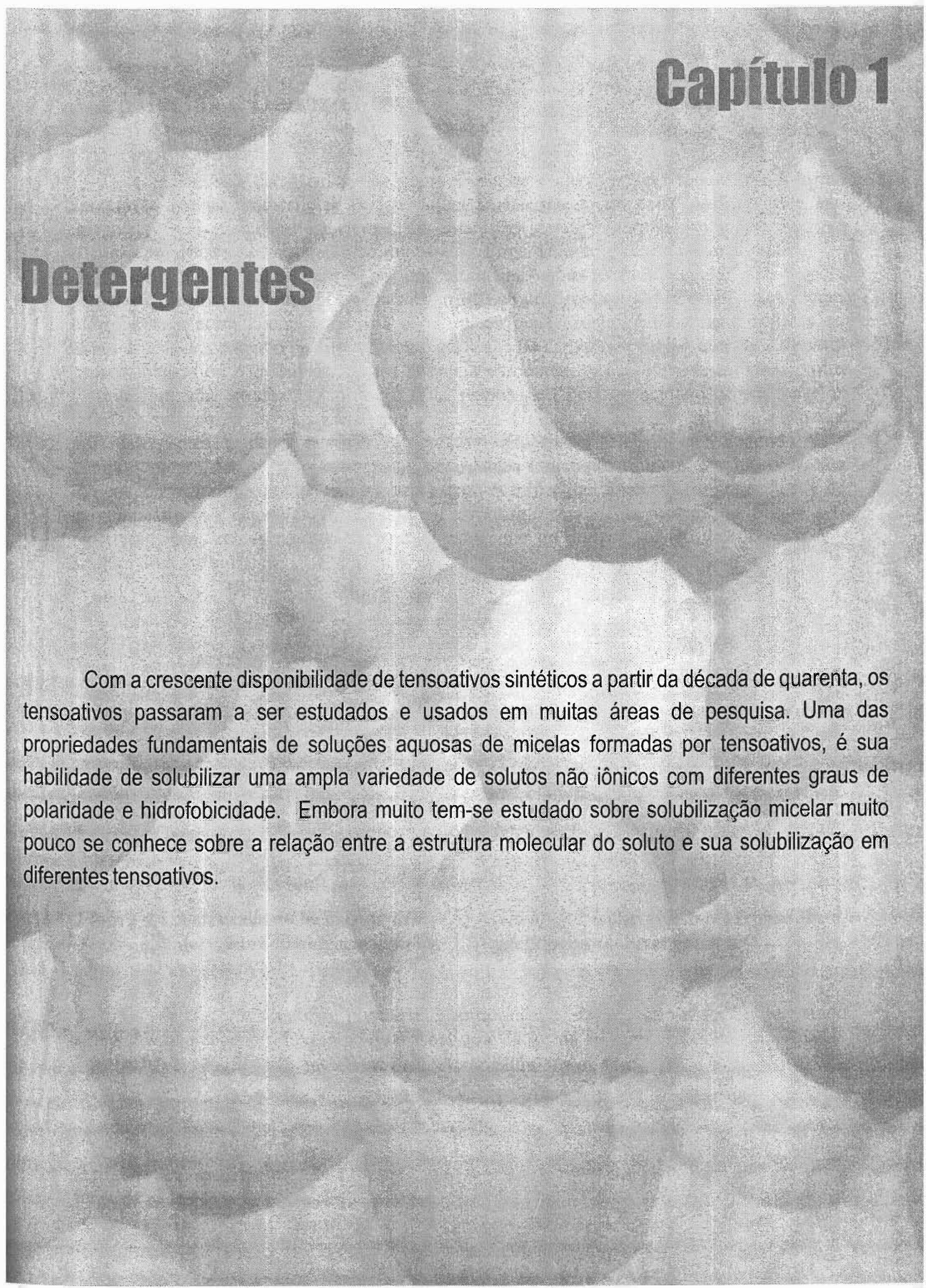




\subsection{Micelas e Detergentes}

Detergentes são substâncias que, em pequenas concentrações, reduzem de forma apreciável a tensão superficial da água ou a tensão interfacial entre dois líquidos não miscíveis. Suas moléculas interferem nas interações existentes entre moléculas de água (ligação por pontes de hidrogênio), diminuindo a força de interação atrativa entre elas.

Os detergentes caracterizam-se por possuírem na sua estrutura molecular duas regiões de polaridade distintas: uma polar (ou hidrofílica) e outra apolar (ou hidrofóbica). A região hidröfóbica é normalmente composta por uma ou mais cadeias carbônicas alquílicas ou alquil-fenílicas, contendo de oito a dezesseis átomos de carbono, a região hidrofílica possui grupos iônicos ou não iônicos ligados à cadeia carbônica. A presença destas duas partes distintas em uma mesma estrutura é o fator responsável pela capacidade de adsorção na interface ar-água ou óleo-água e na superfície dos sólidos. As soluções aquosas formadas por detergentes podem, ou não, apresentar dissociação eletrolítica. Segundo este comportamento, temos, respectivamente, detergentes iônicos (catiônicos e aniônicos), não iônicos e zwitteriônicos (detergentes cuja estrutura molecular, apresenta uma densidade de cargas positivas e negativas, formando uma estrutura formalmente neutra). Na Figura 1.1, mostramos uma representação da estrutura molecular do dodecíl sulfato de sódio ( $S D S-$ sodium dodecyl sulfate).

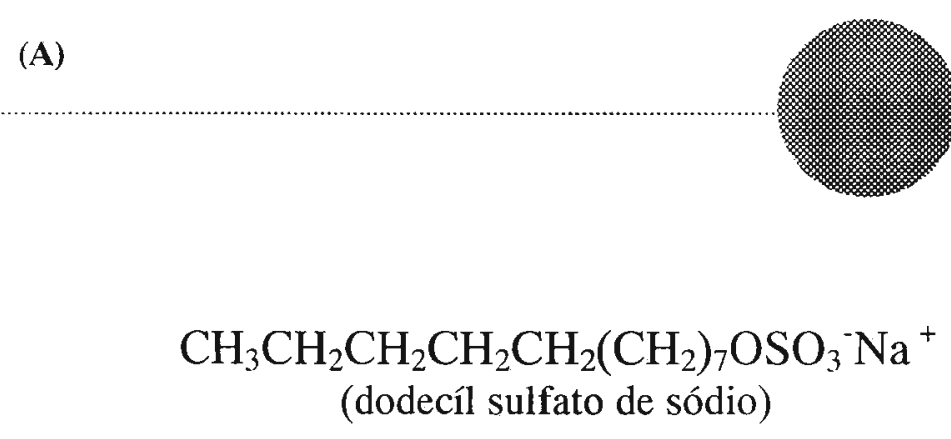

(B)

Figura 1.1 Estrutura molecular do $S D S,(\mathrm{~A})$ parte apolar (solúvel em óleo, gordura e
derivados de hidrocarbonetos), (B) parte polar (solúvel em água).

Os detergentes também possuem a propriedade de se auto-associar formando agregados que dependendo do tipo de tensoativo e da forma de agregação são denominadas micelas $^{\&}$ (veja Figura 1.2), micelas mistas, cristais líquidos ou vesículas.

\& Tipo de agregação de detergentes em um solvente. 


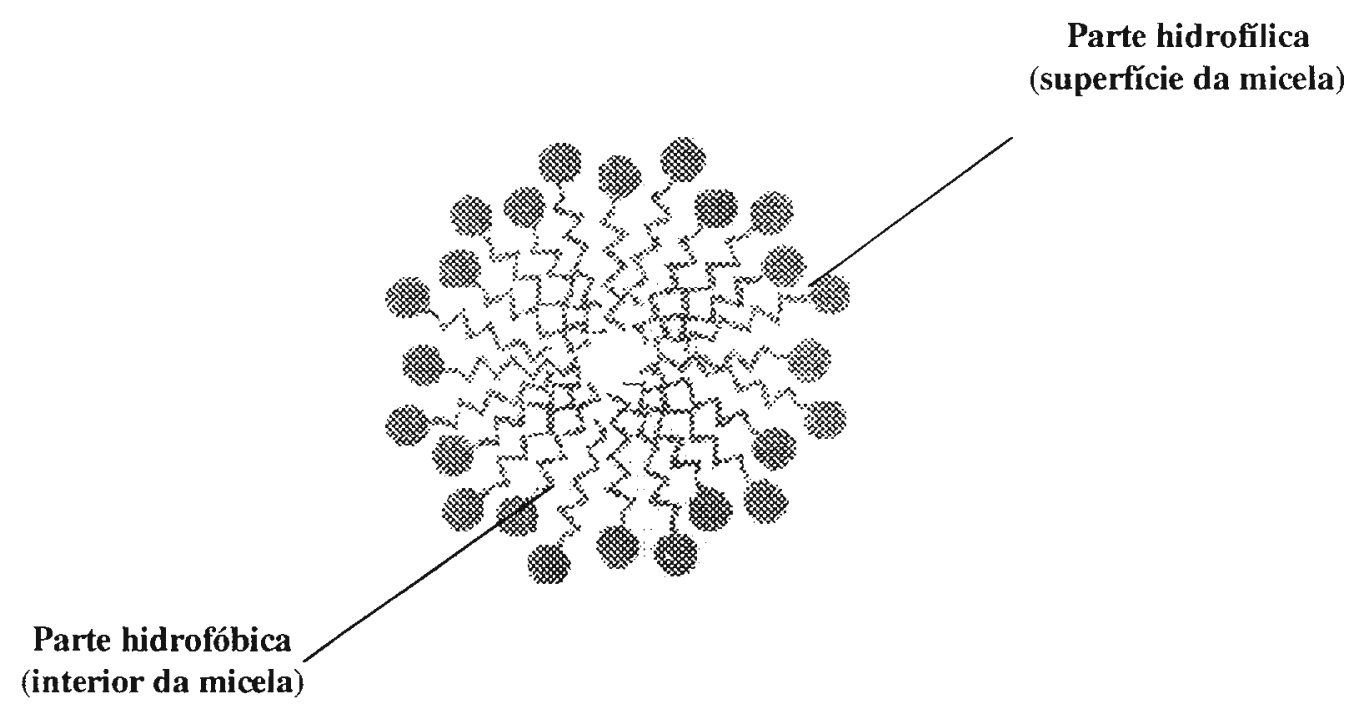

Figura 1.2 Corte transversal de uma micela globular.

Os sistemas micelares apresentam propriedades interessantes de solubilização e é capaz de afetar a velocidade de reações químicas, deslocar equilíbrios e modificar o comportamento de espécies geradas fotoquimicamente. Por causa destas características, estas substâncias são utilizadas como detergentes ${ }^{\%}$, agentes emulsificantes ${ }^{\perp}$, dispersantes ${ }^{\S}$ e solubilizantes ${ }^{\varsigma}$, encontrando atualmente um vasto campo de aplicações industriais. A formação destes agregados ocorre a partir de uma faixa de concentração conhecida como concentração micelar crítica $(c m c)$, na qual observa-se descontinuidade nas propriedades físicas da solução, tais como condutividade, espalhamento de luz, tensão superficial etc. Depois de formados, estes agregados ou micelas são capazes de solubilizar solutos orgânicos com diferentes polaridades e grau de hidrofobicidade.

$\mathrm{Na}$ Figura 1.3 mostramos uma representação esquemática dos possíveis sítios de solubilização em uma micela globular.

Uma das aplicações mais recentes em química analítica de micelas formadas por SDS é em cromatografia eletrocinética ${ }^{1-4}$ (MEKC - Micelar Electrokinetic Chromatography), a qual é usado como separador de importantes compostos biológicos ${ }^{5}$. Surfactantes como $S D S$ também são usados como modificador da fase móvel em cromatografia líquida de alto desempenho (HPLC - High Performance Liquid Chromatography) para alterar a seletividade das separações ${ }^{6 .}$ Micelas também são usadas em espectroscopia de absorção e emissão para melhorar a solubilidade das amostras de complexos metálicos com ligantes $\operatorname{orgânicos}^{7-9}$.

\footnotetext{
"Propriedade de formar micelas detergentes ou óleos dispersos em água.

${ }^{\perp}$ São substâncias ou preparação que favorecem o processo de remoção de sujeira de uma superfície.

São tensorativos usados para manter partículas sólidas em suspensão, em meio líquido.

\$ É a formação de um sistema de pelo menos três componentes: água, um líquido insolúvel em água e detergentes. no qual o líquido insolúvel encontra-se solubilizado.
} 


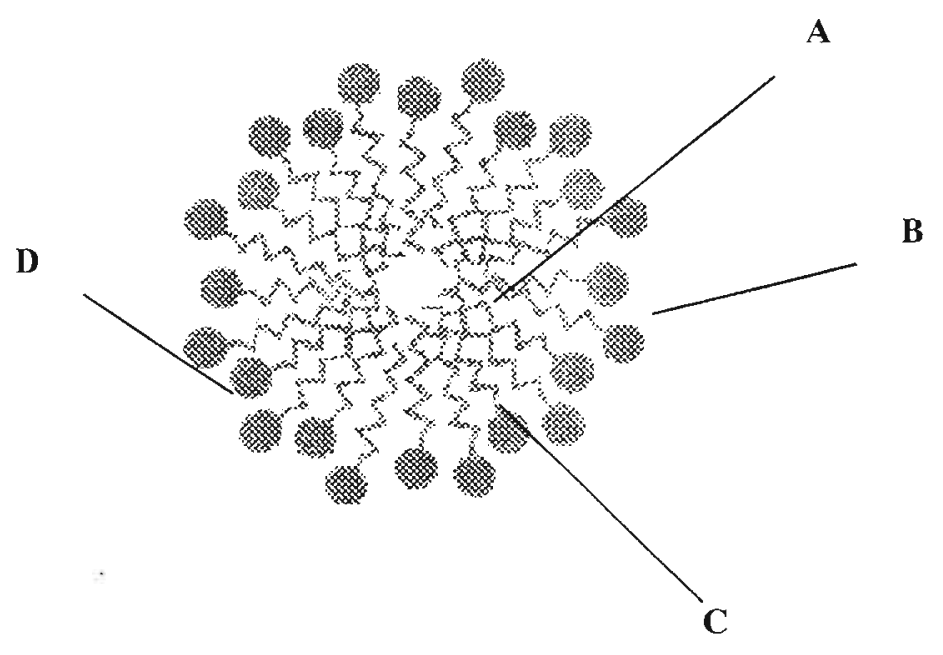

Figura 1.3 Representação esquemática dos possíveis sítios de solubilização na micela: (A) próximo às cadeias hidrocarbônicas; (B) na interface com pequena penetração; (C) na interface com grande penetração; (D) adsorvido na superfície da micela.

Embora muito se tenha estudado sobre solubilização em meio micelar, atualmente pouco se conhece sobre a relação entre a estrutura molecular do soluto e sua solubilização em meio micelar. Diferentemente dos solventes homogêneos, as micelas possuem um ambiente de solubilização mais complexo pelo fato de possuir em sua estrutura duas regiões de polaridade distintas. Essa microheterogeneidade no ambiente de solubilização é um fator importante na determinação da natureza dos vários fatores que contribuem para a solubilização. Solutos com diferentes tipos de estrutura molecular poderiam solubilizar em regiões distintas na micela, assim como compostos com estrutura molecular similar, mas graus diferentes de hidrofobicidade.

No intuito de elucidar o aspecto estrutural da interação de diferentes solutos com a fase micelar, Quina et. al. ${ }^{10,11}$ vem utilizando o modelo de solvatação proposto por Abraham $^{12}$ o qual baseia-se no conceito de relações lineares de energia livre de solvatação. Considerando a heterogeneidade dos solutos quanto a sua estrutura molecular, tamanho, polaridade e capacidade doadora e aceptora de pontes de hidrogênio, este tipo de estudo mostra a possibilidade de determinação dos possíveis ambientes (sítios) de solubilização. Do ponto de vista prático, a análise da correlação linear permite estimar os coeficientes de incorporação de solutos neutros para diferentes detergentes com uma margem de erro estabelecida pela equação de regressão, pois para a maioria das aplicações como catálise e cromatografia micelar, valores aproximados de $K_{s}$ são freqüentemente suficientes.

No próximo capítulo apresentaremos as bases conceituais e a interpretação da relação linear de energia livre de solvatação. 


\section{Referências}

1. Terabe, S.; Otsuka, K.; Ichikawa, K.; Tsuchaia, A.; Ando, T.; 1984, Anal. Chem., 56, 111.

2. Balchunas, A. T.; Swaile, D. F.; Powel, A. C.; Sepaniak, M.; 1988, J. Sep. Sci. Technol., 23, 1891.

3. Terabe, S.; Koji, O.;Ando, T.; 1985, Anal. Chem., 57, 834.

4. Sepaniak, M.; Cole, R. O.; 1987, Anal. Chem., 59, 472.

5. Young, J. K.; Hicks, R. P.; 1994, Biopolymers, 34, 611.

6. Garcia, M. A.; Vera, S.; Bombin, M.; Marina, M. L.; 1993, J. Chromatogr., 34, 123.

7. Cline-Love, L. J.; Habarta, J. G.; Dorsey, J. G.; 1984, Anal. Chem., 56, 1132 A.

8. McIntire, G. L.; 1990, Crit. Rev. Anal. Chem., 21, 257.

9. Hinze, W.; 1979, Solution Chemistry of Surfactants; Mittal, K. L., Ed. Plenum Press: New York.

10. Quina, F. H; Alonso, E. O.; Farah, J. P. S.; 1995, J. Phys. Chem.; 99, 11708.

11. Freitas, A. A.; Quina, F. H.; Carroll, F. A.; 1997, J. Phys. Chem. B,101, 7488.

12. Abraham, M. H.; 1993, Chem. Soc. Rev., 22, 73. 


\section{Canítulo 2 \\ Relação Linear de Energia \\ Livre de Solvatação [LSERs]}

A correlação ea regressão linear múltipla são técnicas destinadas a estimar o relacionamento entre uma variável dependente e duas ou mais variáveis independentes em uma determinada população. A correlação sintetiza o grau de relacionamento entre as variáveis, enquanto a equação de regressão equaciona matematicamente o relacionamento entre a variável dependente e as variáveis independentes. A equação matemática obtida pode ser usada para predizer valores da variável dependente, conhecidos os valores das variáveis independentes.

A análise de regressão linear múltipla é muito útil, quando procuramos determinar quais as variáveis que são potencialmente importantes para um determinado estudo, neste caso, a informação está contida no grau ou na força do relacionamento.

Neste capítulo discutiremos como o método de análise de regressão linear múltipla pode ser usado para descrever a incorporação de solutos não iônicos em micelas aquosas com base no modelo de solvatação da cavidade, empregando parâmetros empíricos como descritore. 


\subsection{Correlação e Regressão Linear Múltipla}

A correlação e a regressão linear múltipla são duas técnicas matemáticas estreitamente relacionadas que envolvem uma forma de estimativa de uma relação que possa existir em uma determinada população. Mais especificamente, o estudo da correlação múltipla compreende a análise de dados amostrais para saber se e como três ou mais variáveis estão relacionadas numa dada população. Como resultado, é obtido um número denominado de coeficiente de correlação $(R)$, o qual indica o percentual de variação da variável (dependente) que é explicada estatisticamente pelas outras variáveis (independentes). Por exemplo, se $(R)$ é igual a 0,81 significa que $81 \%$ da variação dos pontos em torno das médias grupais podem ser explicadas pelas variáveis independentes incluídas na correlação. Inversamente, $(1-R)$, ou $19 \%$ da variação, não pode ser explicada pela relação entre as variáveis e, assim, devemos considerar as variações como sendo devido a outros fatores não inclusos no estudo.

Há diversas maneiras em que as equações de regressão são utilizadas. Uma delas é em situações onde todas as variáveis medem aproximadamente a mesma coisa, mas uma delas é relativamente dispendiosa, ou difícil de lidar, enquanto que outras não. Outra utilização da equação de regressão é explicar valores de uma variável em termos de outras. Utiliza-se este último enfoque para estimar os valores dos coeficientes de incorporação de solutos neutros em micelas aquosas de $S D S$ em função de parâmetros empíricos do soluto obtidos através de medidas experimentais.

É importante reconhecermos certos fatos relativos à equação de regressão. Um ponto importante, é que se trata de uma relação média. Assim, o valor da variável dependente (explicada) não terá necessariamente o valor exato indicado pela equação de regressão. Outro ponto importante é que seria muito arriscado extrapolar os resultados da equação de regressão para dados fora do conjunto usado como amostra. A análise de regressão apenas indica qual relação matemática poderá existir, se existir alguma. Em outras palavras, nem a regressão nem a correlação podem mostrar que uma variável tende a "causar" certos valores em outra variável. O método mais usado para obter a equação de regressão é a técnica dos mínimos quadrados $^{1}$. A denominação provém do fato de a reta resultante minimizar a soma dos quadrados dos desvios dos pontos em relaçāo à reta. A obtenção da equação da reta é efetuada com auxílio de programas de tratamento estatístico.

\subsection{Correlação e Causalidade}

Quando três ou mais variáveis estão correlacionadas, é possível predizer valores de uma delas com base no conhecimento das outras. Isso leva freqüentemente à conclusão de que uma variável é causa da outra. Isso é verdadeiro somente quando as variáveis explicativas (ou independentes) precedem a variável explicada no tempo. Entretanto, o fato de haver um relacionamento matemático entre as variáveis nada nos diz quanto à causa e o efeito. Logo, há três explicações plausíveis para a obtenção de uma correlação: (a) existe uma relação de causa e efeito; (b) as variáveis acham-se relacionadas com uma outra variável; e (c) a correlação é devida ao acaso. O perigo da utilização para fins de previsão de relações lineares que não tenham sido validadas em termos de causa e efeito é que modificações na variável independente possam não conduzir às modificações esperadas na variável dependente devido à presença de outros fatores não contemplados pela relação matemática obtida. 
A analise da regressão envolve inferências quanto ao verdadeiro relacionamento existente na população, ou seja, a quão precisa é as diversas estimativas de regressão. A determinante principal da precisão é descrita pela quantidade de dispersão existente na população sendo descrito pelo desvio padrão $(s d)$ da distribuição dos pontos em torno da reta de regressão, ou seja, quanto menor o desvio padrão mais precisa será as estimativas feitas usando-se a equação de regressão. O teste estatístico $(F)$, serve como um critério padrão para teste de hipóteses em análise de regressão linear múltipla. $F$ pode ser expresso em termos de $\left(R^{2}\right)$ modulado pelo número de graus de liberdade:

$$
F=\left(R^{2} / k\right) /\left[\left(1-R^{2}\right) /(n-k-1)\right]
$$

Onde $k$ é o número de variáveis explicativas (independentes) usadas no modelo e $n$ o número de dados usados no estudo. Em outras palavras, $F$ é um critério para comparar a significância da regressão linear múltipla com a alternativa de hipótese nula (a qual assume que nenhuma variável explicativa correlaciona com a variável explicada). Quanto maior o valor de $F$ mais coerente é o modelo proposto.

\subsection{Relação Linear de Energia Livre de Solvatação.}

Um dos principais instrumentos extensivamente usado em estatística é o modelo. Os modelos são versões simplificadas que permitem estudar um problema ou situação real. São usados para ilustrar certos aspectos do problema em estudo, evitando grande número de detalhes que talvez sejam irrelevantes, podendo ainda reduzir o grau de complexidade do problema em estudo. Neste intuito, as relações quantitativas de estrutura atividade (QSAR Quantitative Structure Activity Relationships) ${ }^{2}$ são usadas extensivamente para relacionar propriedades estruturais e eletrônicas com atividade biológica. A filosofia $Q S A R$ estabelece que existe uma relação quantitativa entre propriedades microscópicas (estrutura molecular) e macroscópicas particularmente atividades biológicas de compostos. O modelo correlaciona valores de atividades biológicas de uma determinada série de compostos com suas propriedades físico-químicas através de regressão linear múltipla. Similarmente relações quantitativas de estrutura-propriedade podem ser usadas para relacionar aspectos da estrutura molecular com propriedades físico-químicas. Em $1937 \mathrm{Hammet}^{3}$, empregando um formalismo idêntico, propôs a equação que leva seu nome. Essa e outras equações que descrevem relações lineares são chamadas de relações lineares de energia livre (LFER - Linear Free Energy Relationship). Além de $Q S A R$ e $L F E R$, outra relação linear de energia livre de solvatação (LSER-Linear Solvation Energy Relationship) foi desenvolvida por Kamlet, Taft e Abboud ${ }^{4}$ para explicar efeitos de solventes, onde o solvente é caracterizado por constantes que descrevem sua habilidade de doar e receber pontes de hidrogênio e sua dipolaridade/polarizabilidade. Os valores dos parâmetros usados nas relações lineares foram originalmente obtidos através das medidas espectroscópicas no UV e são chamados de parâmetros solvatocrômicos. Baseado no modelo de solvatação da cavidade, Abraham et. al. ${ }^{5-}$ ${ }^{8}$ propuseram uma relação linear de energia livre de solvatação (LSER) que se baseiam em parâmetros solvatocrômicos específicos do soluto. Esta metodologia utiliza um único conjunto de parâmetros do soluto para correlacionar com sucesso uma ampla faixa de propriedades físicas, químicas e biológicas. A seguir, descreveremos o modelo de solvatação 
da cavidade e a correspondente equação linear de onde provem o nome relação linear de energia livre de solvatação.

\subsection{Modelo de Solvatação da Cavidade}

O modelo de solvatação da cavidade de Abraham para a solubilização abrange os seguintes estágios: (a) formação de uma cavidade adequada ao tamanho do soluto no solvente; (b) introdução do soluto na cavidade e (c) reorganização das moléculas do solvente ao redor da cavidade. Estes estágios são descritos pela equação linear geral que descreve os três estágios da solubilização como segue:

Propriedade $=$ Constante + Termo da Cavidade + Termos de Dipolaridade $/$ Polarizabilidade + + Termos de Interação por Pontes de Hidrogênio

Os termos da equação (2.2) podem ser interpretados em termos microscópicos (energéticos) e em termos macroscópicos (termodinâmicos). Microscopicamente o termo de cavidade mede a energia necessária para vencer as forças de coesão entre as moléculas do solvente para formar a cavidade para o soluto. O termo de dipolaridade/polarizabilidade mede a energia das interações de dipolo-dipolo e dipolo induzido entre as moléculas do solvente e soluto que contribuem para a formação da solução. Os termos de pontes de hidrogênio medem a energia das interações quando um complexo soluto-solvente é formado.

A interpretação macroscópica ou termodinâmica pode ser inferida pelo trabalho de Abraham et. al. ${ }^{8}$ A energia livre necessária para gerar a cavidade no solvente vai depender das forças de coesão entre as moléculas do solvente e do tamanho da cavidade. Uma maneira de medir a força entre as moléculas do solvente é proporcionada pelo parâmetro de densidade de energia coesiva de Hildebrand $\left(\delta_{I I}\right)_{1}^{2}$, definido como a entalpia de vaporização $\left(\Delta H_{v}\right)$ do solvente a $298 \mathrm{~K}$ por unidade de volume de moléculas do solvente $\left(V_{l}\right)$, sendo $R$ a constante universal dos gases e $T$ a temperatura absoluta ${ }^{9-11}$,

$$
\left(\delta_{I I}\right)_{1}^{2}=\frac{\left(\Delta H_{v}-R T\right)}{V_{1}}
$$

Numa primeira aproximação, o tamanho da cavidade pode ser considerado como proporcional ao volume molar do soluto $V_{2}$. A segunda consideração é que a energia de reorganização das moléculas do solvente ao redor da cavidade não é muito importante comparado com a energia necessária para formar a cavidade. Por último, o processo de incorporação do soluto na cavidade envolve interações gerais de dispersão (proporcionais ao volume $V_{2}$ ), bem como outras interações que dependem da natureza do soluto e do solvente.

Interaçōes do tipo ácido-base ocorrem quando o solvente tem grupo funcional formadores de pontes de hidrogênio (de caráter "ácido" $\alpha_{l}$ ) e o soluto, grupos aceptores de pontes de hidrogênio (de caráter "básico" $\Sigma \beta_{2}$ ) ou se o solvente é um aceptor $\left(\beta_{1}\right)$ e o soluto um doador $\left(\Sigma \alpha_{1}\right)$ de pontes de hidrogênio. Além do mais, podem existir interações do tipo dipolo-dipolo e dipolo induzido, as quais são proporcionais as dipolaridades do solvente $\left(\pi_{1}\right)$ e soluto $\left(\pi_{2}\right)$ e interações proporcionais à refração molar do soluto $\left(R_{2}\right)$ e do solvente $\left(\delta_{1}\right)$. 
Considerando estes termos, Abraham et. al. ${ }^{8}$ propuseram uma equação geral para a solução de um soluto num solvente:

$$
\Delta G^{0}=\Delta G_{0}^{0}+a_{1} \delta_{1} R_{2}+a_{2} \pi_{1} \pi_{2}+a_{3} \alpha_{1} \sum \beta_{2}+a_{4} \beta_{1} \sum \alpha_{2}+a_{5} \delta_{1}^{2} V_{2}
$$

onde $a_{1}, a_{2}, a_{3}, a_{4}$ e $a_{5}$ correspondem a constantes de proporcionalidade e os índices 1 e 2 referem-se ao solvente e soluto, respectivamente. No caso de uma série de solutos num único solvente, todas as características relativas ao solvente são constantes na equação (2.4), obtendo-se a seguinte expressão para a energia livre de solvatação de solutos:

$$
\Delta G^{0}=a_{0}+a_{1} R_{2}+a_{2} \pi_{2}+a_{3} \sum \beta_{2}+a_{4} \sum \alpha_{2}+a_{5} V_{2}
$$

$\mathrm{Na}$ relação linear de energia livre de solvatação proposta por Abraham (LSER) a propriedade de interesse $(S P)$ é freqüentemente descrita pelo logaritmo da propriedade medida (que envolve interações do soluto-solvente) que, por sua vez, é relacionada à energia livre de Gibbs.

$$
\log S P=a_{0}+a_{1} R_{2}+a_{2} \pi_{2}+a_{3} \sum \beta_{2}+a_{4} \sum \alpha_{2}+a 5^{(} V_{x} / 100^{\prime}
$$

Os parâmetros empíricos são obtidos como segue; A refração molar em excesso $\left(R_{2}\right)$ é determinada através do índice de refração do soluto ${ }^{12}$. O parâmetro $\left(\pi_{2}\right)$ que descreve a dipolaridade/polarizabilidade pode ser obtido a partir de experiências de cromatografia líquida gasosa ${ }^{6,13,14}$ para compostos voláteis ou através de medidas de coeficiente de partição águasolvente para solutos em geral. Os parâmetros que descrevem a capacidade aceptora $\left(\Sigma \beta_{2}\right)$, e doadora $\left(\sum \alpha_{2}\right)$ de pontes de hidrogênio são obtidos através de medidas de coeficiente de partiçăo $^{15}$ e experiência de cromatografia ${ }^{16}$ respectivamente. $O$ parâmetro $\left(V_{x}\right)$, volume molar do soluto, pode ser calculado a partir da estrutura molecular do soluto pelo método de McGowan ${ }^{17}$.

Além da constante $\left(a_{0}\right)$, dependente da propriedade de interesse e da escolha do estado padrão, a equação contém cinco parâmetros específicos do soluto, insensíveis ao meio, cuja contribuição relativa é ditada pela magnitude dos cinco coeficientes numéricos $\left(a_{1}, a_{2}, a_{3}, a_{4}\right.$, e $a_{5}$ ). Para uma série de solutos num único solvente, podemos interpretar a equação (2.6) da seguinte forma:

Os parâmetros empíricos do soluto representam o efeito do soluto nas interações solutosolvente e os coeficientes representam o efeito ponderado do solvente (pois na realidade temos dois solventes micela e água). Logo nos coeficientes está contida a informação química relacionada ao solvente em estudo. O coeficiente $\left(a_{1}\right)$, descreve a tendência do solvente interagir com o soluto via elétrons- $\pi$. $O$ coeficiente $\left(a_{2}\right)$, descreve a tendência do solvente interagir com solutos polar ou polarizável. O coeficiente $\left(a_{3}\right)$, descreve o caráter básico do solvente "basicidade" (por que solutos com caráter ácido interagem com solventes de caráter básico), e o coeficiente $\left(\mathrm{a}_{4}\right)$, descreve o caráter ácido do soluto "acidez" (por que solutos com caráter básico interagem com solventes de caráter ácido). O coeficiente ( $\left.a_{5}\right)$, descreve as interações de dispersão e a energia envolvida no processo de criar uma cavidade no solvente do tamanho do soluto. A basicidade e a acidez referem-se a capacidade de doar ou receber prótons de moléculas vizinhas de acordo com a definição de ácido-base de Brønsted-Lowry. 
Esse conjunto de parâmetros de solubilizações mostrou-se ser bastante útil para compreender os processos que envolvem transferência de solutos entre duas fases condensadas, como por exemplo, a partição de solutos não iônicos em água-octanol ${ }^{5,6}$. Na Figura 2.1, mostramos um esquema representando o significado físico-químico dos parâmetros empíricos $R_{2}, \pi_{2}, \Sigma \beta_{2}, \Sigma \alpha_{2}$ e $V_{x}$ frente à transferência de um soluto da fase gasosa para um solvente qualquer.

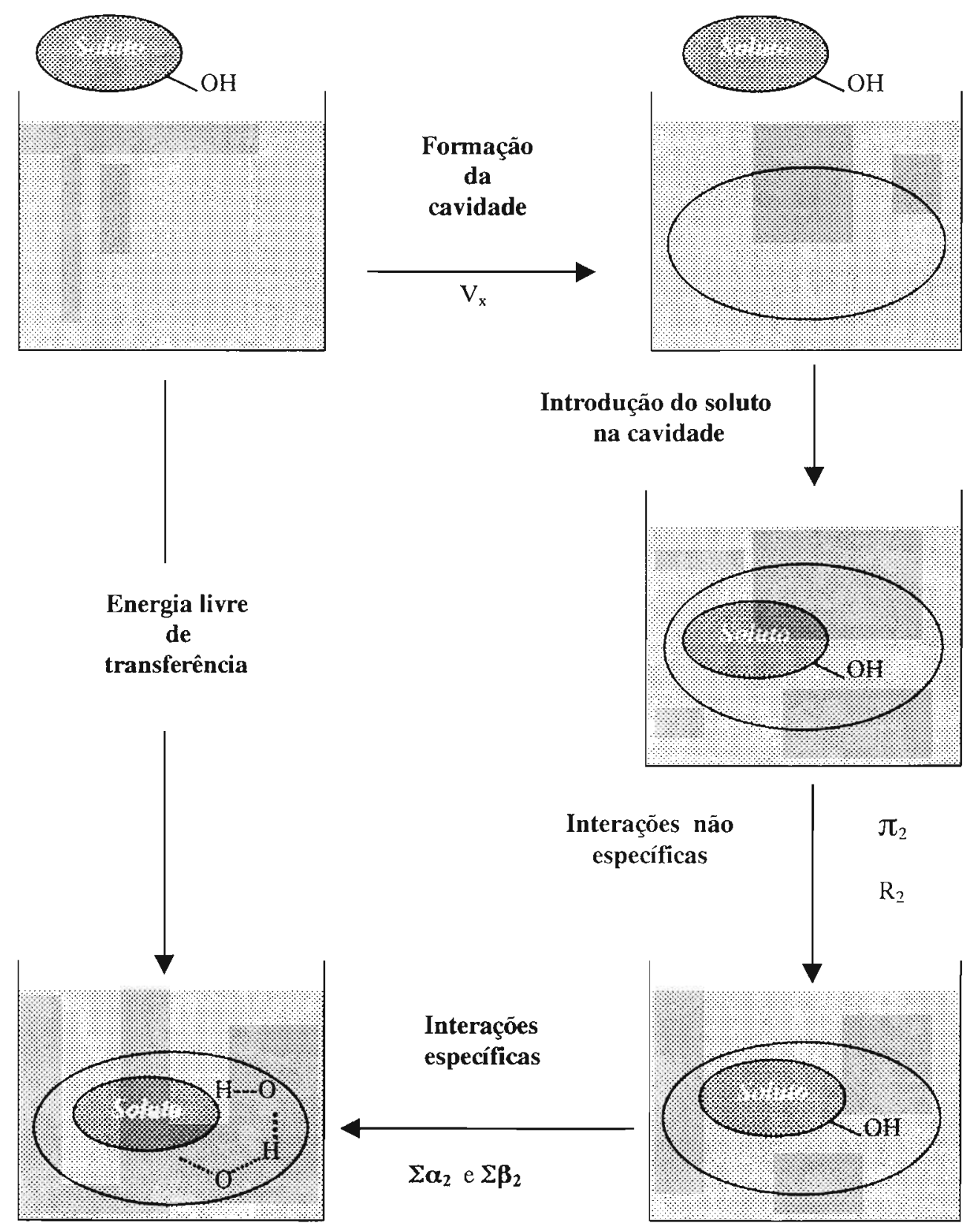

Figura 2.1 Significado físico-químico dos parâmetros do soluto de Abraham inerentes á energia livre de solvatação (LSERs). 
A seguir, mostraremos a aplicação desta metodologia ao caso da incorporação de solutos não iônicos em micelas formadas por (SDS).

\subsection{Aplicação da Relação Linear de Energia Livre de Solvatação em Sistemas Micelares}

Relações de energia livre de solvatação têm provado ser úteis no entendimento de processos que envolvam transferência de solutos entre duas fases condensadas. Isso levounos a examinar a aplicação da equação (2.6) em problemas de solubilização micelar. Para esse propósito, foram compilados da literatura valores da constante de incorporação $\left(K_{s}\right)$ para solutos neutros em solução água-micela de $S D S$. Os valores de $K_{\mathrm{s}}$ e dos parâmetros do soluto de Abraham são mostrados na Tabela 2.1. Os solutos estudados cobrem uma ampla faixa de diferentes tipos de estruturas químicas, incluindo gases simples e hidrocarbonetos alifáticos (6 solutos), compostos halogenados (6 solutos), hidrocarbonetos aromáticos e derivados (11 solutos), álcoois alifáticos (14 solutos), fenóis e naftóis (11 solutos), cetonas (8 solutos), éteres ( 1 soluto), ácidos carboxílicos e derivados ( 7 solutos).

Tabela 2.1 Valores experimentais de $\log \left(K_{s}\right)$ e dos parâmetros empíricos de Abraham para a incorporação de (66) solutos não iônicos em micelas aquosas formadas por $S D S$. Os critérios adotados para a obtenção dos valores de $K_{s}$ estão descritos na referência ${ }^{18}$.

\begin{tabular}{|c|c|c|c|c|c|c|c|}
\hline No & Soluto & ${ }^{2} \log K_{s}$ & $\mathrm{R}_{2}$ & $\pi_{2}$ & $\Sigma \alpha_{2}$ & $\Sigma \beta_{2}$ & $\mathrm{~V}_{\mathrm{x}} / 100$ \\
\hline \multicolumn{8}{|c|}{ Alcoois } \\
\hline s324 & Metanol & $-0,574$ & 0,278 & 0,440 & 0,430 & 0,470 & 0,308 \\
\hline$\$ 325$ & Eianol & -0.125 & 0.246 & 0.420 & 0.370 & 0.480 & 0.449 \\
\hline s326 & 1-Propanol & 0,308 & 0,214 & 0.420 & 0,370 & 0,480 & 0,590 \\
\hline s327 & 2-l'ropanol & 0,146 & 0,212 & 0.360 & 0,330 & 0.560 & 0.590 \\
\hline s328 & I-Butanol & 0,724 & 0,224 & 0.420 & 0,370 & 0,480 & 0,731 \\
\hline$\$ 330$ & 2-Butanol & 0.477 & 0,217 & 0.360 & 0.330 & 0.560 & 0.731 \\
\hline s331 & 2-Metil-2-Propanol & 0,663 & 0,180 & 0.300 & 0.310 & 0,600 & 0.731 \\
\hline$\$ 332$ & 1-Pentanol & 1,224 & 0,219 & 0,420 & 0,370 & 0,480 & 0,872 \\
\hline$\$ 336$ & 3-Metil-1-Butanol & 1,161 & 0,192 & 0.390 & 0,370 & 0,480 & 0,872 \\
\hline $\mathrm{s} 340$ & 1-Hexanol & 1,624 & 0,210 & 0.420 & 0,370 & 0,480 & 1,013 \\
\hline s341 & 2-Hexanol & 1,591 & 0,187 & 0.360 & 0,330 & 0,560 & 1,013 \\
\hline s347 & l-Heptanol & 2,071 & 0,211 & 0.420 & 0,370 & 0.480 & 1.154 \\
\hline $\mathrm{s} 351$ & l-Octanol & 2,437 & 0,199 & 0.420 & 0,370 & 0,480 & 1,295 \\
\hline s653 & Álcool Benzílico & 0,996 & 0,803 & 0,870 & 0,330 & 0,560 & 0,916 \\
\hline \multicolumn{8}{|c|}{ Gases e Hidrocarbonetos Alifáticos } \\
\hline s010 & Oxigènio & $-0,148$ & 0,000 & 0.000 & 0.000 & 0,000 & 0.183 \\
\hline s009 & Argònio & $-0,137$ & 0,000 & 0.000 & 0,000 & 0,000 & 0.190 \\
\hline s015 & Metano & 0,079 & 0,000 & 0,000 & 0,000 & 0,000 & 0,250 \\
\hline s016 & Etano & 0,792 & 0,000 & 0.000 & 0,000 & 0,000 & 0,390 \\
\hline s017 & Propano & 1,342 & 0,000 & 0.000 & 0,000 & 0,000 & 0,531 \\
\hline
\end{tabular}


Tabela 2.1 (continuação)

\begin{tabular}{|c|c|c|c|c|c|c|c|}
\hline$\overline{\text { No }}$ & Soluto & ${ }^{2} \log \mathrm{K}_{\mathrm{s}}$ & $\mathrm{R}_{2}$ & $\pi_{2}$ & $\Sigma \alpha_{2}$ & $\Sigma \beta_{2}$ & $\mathrm{~V}_{\mathrm{x}} / 100$ \\
\hline 5049 & Ciclohexano & $2 . \overline{431}$ & 0.305 & 0,000 & 0.000 & 0.000 & $0,84,5$ \\
\hline \multicolumn{8}{|c|}{ Hidrocarbonetos Aromáticos e Derivados } \\
\hline s389 & Benzeno & 1,354 & 0,610 & 0.520 & 0,000 & 0,140 & 0.716 \\
\hline 5564 & Vitrobenzeno & 1.356 & 0,871 & 1,110 & 0,000 & 0,280 & 0.891 \\
\hline s465 & Clorobenzeno & 1,841 & 0.718 & 0.6 .50 & 0,000 & 0,070 & 0.839 \\
\hline s390 & Tolueno & 1,733 & 0,601 & 0.520 & 0,000 & 0,140 & 0,857 \\
\hline s391 & Etilbenzeno & 2,438 & 0,613 & 0.510 & 0,000 & 0,150 & 0,998 \\
\hline s394 & p-Xileno & 2,209 & 0,613 & 0.520 & 0.000 & 0,160 & 0,998 \\
\hline $5490^{-}$ & Anisole & 1.462 & 0,708 & 0.750 & 0,000 & 0,290 & 0,916 \\
\hline$s 423$ & Naftaleno & 2,566 & 1,340 & 0,920 & 0,000 & 0,200 & 1,085 \\
\hline $\mathrm{s} 424$ & 1-Metilnaltaleno & 3,064 & 1,344 & 0,900 & 0,000 & 0,200 & 1,226 \\
\hline s421 & Bifenil & 3,096 & 1,360 & 0,990 & 0,000 & 0,220 & 1,324 \\
\hline$s 443$ & Antraceno & 3,772 & 2,290 & 1,340 & 0,000 & 0,260 & $1,4.54$ \\
\hline \multicolumn{8}{|c|}{ Ácidos Carboxílicos e Derivados } \\
\hline s576 & Acetanilida & 1,255 & 0,870 & 1.400 & 0,500 & 0,670 & 1,113 \\
\hline $\mathrm{s} 250$ & 1- Cianopropano & $0,6.33$ & 0,188 & 0.900 & 0,000 & 0,360 & 0,686 \\
\hline s580 & Ácido Benzóico & 1,560 & 0.730 & 0.900 & 0,590 & 0,400 & 0,932 \\
\hline s569 & Benzamida & 0,882 & 0,990 & 1,500 & 0,490 & 0,670 & 0,973 \\
\hline s532 & Benozonitrila & 1,387 & 0,742 & 1.110 & 0,000 & 0,330 & 0,871 \\
\hline s583 & 4-Metíl Ácido Benzóico & 1,851 & 0,730 & 0,900 & 0,600 & 0,380 & 1,073 \\
\hline $\mathrm{s} 582$ & 3-Metíl Ácido Benzóico & 1,919 & 0,730 & 0,900 & 0.590 & 0.380 & 1,073 \\
\hline \multicolumn{8}{|c|}{ Éteres } \\
\hline s169 & 1.4-Dioxano & 0,114 & 0,329 & 0.750 & 0,000 & 0,640 & 0.681 \\
\hline \multicolumn{8}{|c|}{ Compostos Halogenados } \\
\hline s094 & Diclorometano & 0,556 & 0,387 & 0.570 & 0,100 & 0,050 & 0,494 \\
\hline s095 & Cloroformio & 1,025 & 0,425 & 0.490 & 0,150 & 0,020 & 0,617 \\
\hline s096 & Tetraclorometano & 1,548 & 0,458 & 0.380 & 0,000 & 0,000 & 0,739 \\
\hline s144 & Iodoetano & 1.342 & 0.640 & 0.400 & 0.000 & 0,150 & 0,649 \\
\hline s15i & Halotano & 1,057 & 0,102 & 0.380 & 0.150 & 0,050 & 0.741 \\
\hline$\$ 140$ & 1-lodobutano & 2.057 & 0.628 & 0.400 & 0,000 & 0.150 & 0.930 \\
\hline \multicolumn{8}{|c|}{ Aldeídos e Cetonas } \\
\hline s181 & Propanona & $-0,045$ & 0,179 & 0.700 & 0,040 & 0,490 & 0.547 \\
\hline s182 & 2-Butanona & 0,327 & 0,166 & 0.700 & 0,000 & 0,510 & 0,088 \\
\hline s183 & 2-Pentanona & 0,799 & 0,143 & 0,680 & 0,000 & 0,510 & 0,829 \\
\hline s186 & 2-Hexanona & 1,235 & 0,136 & 0,680 & 0,000 & 0,510 & 0,970 \\
\hline s190 & 2-Heptanona & 1,676 & 0,123 & 0.680 & 0,000 & 0,510 & 1,111 \\
\hline s506 & Acetofenona & 1,522 & 0,818 & 1.010 & 0,000 & 0,480 & 1,014 \\
\hline $\mathrm{s} 513$ & Benzofenona & 2,653 & 1,447 & 1.500 & 0,000 & 0,500 & 1,481 \\
\hline s512 & Etilfenil Cetona & 1,732 & 0,804 & 0.950 & 0,000 & 0,510 & 1,155 \\
\hline \multicolumn{8}{|c|}{ Aminas Aromáticas } \\
\hline s536 & Anilina & 0,676 & 0,955 & 0.960 & 0.260 & 0,500 & 0,860 \\
\hline s539 & $p$-Toluidina & 1,278 & 0,923 & 0.950 & 0.230 & 0.520 & 0.957 \\
\hline \multicolumn{8}{|c|}{ Fenóis e Naftóis } \\
\hline$s 589$ & Fenol & 0,967 & 0,805 & 0,890 & 0,600 & 0,300 & 0,775 \\
\hline s623 & t-Fluorofenol & 1,204 & 0,670 & 0.970 & 0,630 & 0,230 & 0,793 \\
\hline S626 & 4-Clorofenol & 1,583 & 0,915 & 1,080 & 0,670 & 0,200 & 0,898 \\
\hline s633 & 4-Bromofenol & 1,826 & 1,080 & 1.170 & 0,670 & 0,200 & 0,950 \\
\hline
\end{tabular}


Tabela 2.1 (continuação)

\begin{tabular}{|c|c|c|c|c|c|c|c|}
\hline$\overline{\text { No }}$ & Soluto & ${ }^{3} \log K_{s}$ & $\mathrm{R}_{2}$ & $\pi_{2}$ & $\sum \alpha_{2}$ & $\Sigma \beta_{2}$ & $\overline{V_{x} / 100}$ \\
\hline$\overline{\mathrm{s636}}$ & 4-lodofenol & 2,089 & 1,380 & 1,220 & 0,680 & 0.200 & $1,0 \overline{33}$ \\
\hline$s 645$ & 4-Nitrofenol & 1,284 & 1,070 & 1,720 & 0,820 & 0,260 & 0,949 \\
\hline s590 & $o$-Cresol & 1,330 & 0,840 & 0,860 & 0,520 & 0,300 & 0,916 \\
\hline s591 & $m$-Cresol & 1,348 & 0,822 & 0,880 & 0,570 & 0,340 & 0.916 \\
\hline s592 & $p$-Cresol & 1,372 & 0,820 & 0,870 & 0,570 & 0,310 & 0,916 \\
\hline s651 & 1-Naftol & 2,017 & 1,520 & 1,050 & 0,610 & 0,370 & 1,144 \\
\hline $\mathrm{s} 652$ & 2-Naftol & 2,232 & 1,520 & 1,080 & 0,610 & 0,400 & 1,144 \\
\hline
\end{tabular}

As unidades de $R_{2}$ e $V_{x}$ são expressas em $\mathrm{cm}^{3} \mathrm{~mol}^{-1} / 10 \mathrm{e} \mathrm{cm}^{3} \mathrm{~mol}^{-1}$ respectivamente. a $O s$ valores de $\log \left(K_{\mathrm{s}}\right)$, são a média dos valores experimentais compilados na referência 18 . Os valores $\operatorname{de} \Sigma \beta_{2}$ para a anilina e $p$-toluidina foram substituídos por valores alternativos $\operatorname{de} \Sigma \beta_{2}{ }^{013}$.

O resultado da análise por regressão linear múltipla para o conjunto de dados da Tabela 2.1 mostra que a correlação expressa pela equação (2.7) permite estimar o coeficiente de incorporação micelar com precisão de um fator de dois se conhecermos os parâmetros experimentais de Abraham.

O coeficiente de correlação $(R)$, o qual determina o grau de correlação entre os parâmetros, o desvio padrão $(s d)$ e o valor de $(F)$ da equação de regressão são significativos para a amostragem de 66 solutos não iônicos.

$$
\begin{gathered}
\log \left(K_{s}\right)=-0,62+0,32 R_{2}-0,57 \pi_{2}-1,84 \sum \beta_{2}-0,08 \sum \alpha_{2}+3,25^{(} V_{x} / 100^{\prime} \\
(S D S: n=66 ; R=0,989 ; s d=0,13 ; F=575)
\end{gathered}
$$

O coeficiente de correlação múltipla mostra que existe uma boa correlação entre as variáveis; apenas $2 \%$ das estimativas não podem ser explicadas pela variação nos parâmetros escolhidos como variáveis independentes.

Uma boa correlação é necessária, porém não é uma condição suficiente para a validade da metodologia. A magnitude e sinal dos coeficientes devem também ser compatíveis com o processo de incorporação. Analisando a equação (2.7), as contribuições dominantes para o processo de incorporação do soluto vem dos termos $V_{x}$ e $\Sigma \beta_{2}$. O grande valor positivo do coeficiente do termo $V_{x}$ significa que é mais fácil criar uma cavidade na micela do que na água, refletindo a energia de coesão maior da água. Analisando a Tabela 2.1, verifica-se que, para os hidrocarbonetos alifáticos, todos os parâmetros são zero exceto $V_{x}$. Conseqüentemente, o coeficiente de $V_{x}$ deve conter o efeito de hidrofobicidade expresso em termos do volume do soluto (ao invés da área da superfície de contato entre o soluto e a água), como notado por Quina et. al. ${ }^{18}$. O elevado valor do coeficiente do termo $\Sigma \beta_{2}$ implica que interações de ponte de hidrogênio entre o soluto como aceptor e a fase como doadora favorece a incorporação do soluto na fase aquosa, isto é, a fase aquosa é melhor doadora de pontes de hidrogênio do que os possíveis sítios de solubilização na micela. Em parte, isso se deve à menor concentração local de doadores por pontes de hidrogênio no sítio de solubilização da micela. Alternativamente, na região de contato hidrocarboneto-água, a água tem uma grande tendência de manter suas pontes de hidrogênio intactas ${ }^{19}$. Como conseqüência, na interface micela-água deve existir uma tendência a favorecer arranjos das moléculas de água em que as ligações $\mathrm{O}-\mathrm{H}$ se orientam tangencialmente à interface ou com os átomos de hidrogênio 
apontando na direção oposta a interface, isto é, na direção da fase aquosa. Orientações desse tipo reduziriam o caráter ácido das pontes de hidrogênio na interface micela-água relativo às na fase aquosa.

A equação (2.7) também contém a informação de que, no caso de micelas formadas por $S D S$, a incorporação micelar é muito pouco influenciada pelo termo de dipolaridade $\pi_{2}$, indicando que a dipolaridade da fase aquosa é modestamente maior que nos sítios de solubilização da micela. A relativa insensibilidade de $K_{s}$ em relação a dipolaridade é atribuída ao fato de que solutos com valor significante de $\pi_{2}$ preferencialmente solubilizam nas proximidades da interface micela-água (veja Figura 1.3). A fraca dependência de $K_{s}$ em relação a $R_{2}$ é atribuída ao fato de que nem a água, nem a micela formada por esse detergente, fornecem ambientes de solubilização polarizáveis. As pontes de hidrogênio de caráter ácido do soluto não contribuem ou contribuem muito pouco para a solubilização micelar, indicando que a basicidade do sítio de solubilização micelar é comparável com a da fase aquosa. Essa interpretação é consistente com a orientação preferencial das ligações $\mathrm{O}-\mathrm{H}$ das moléculas de água na interface micela-água sugerida acima. Na Figura 2.2 mostramos uma comparação entre os valores de $\log \left(K_{\mathrm{s}}\right)$ determinados através da equação (2.7) e obtidos partir de resultados experimentais.

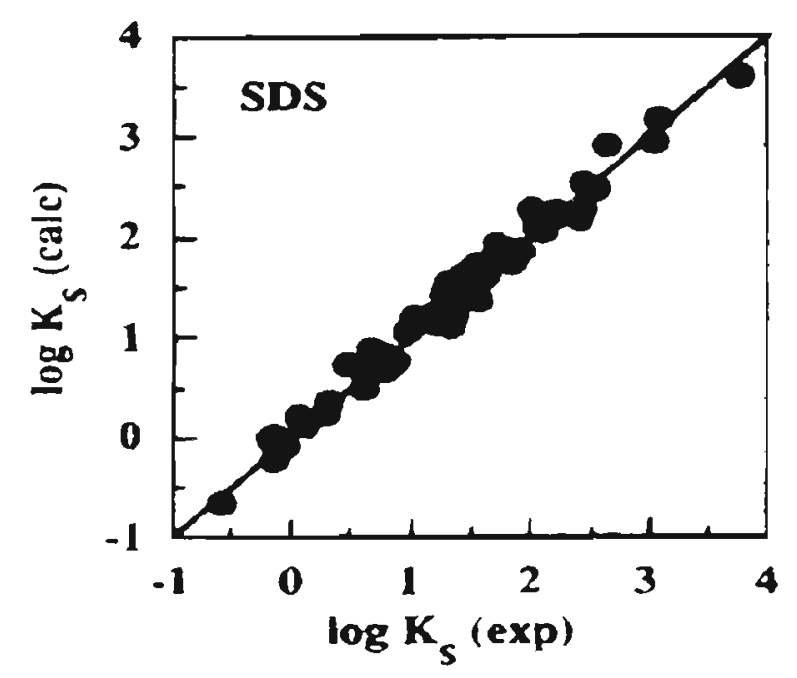

Figura 2.2 Comparação entre valores experimentais de $\log \left(K_{s}\right)$ e calculados através da equação (2.7) para a incorporação de (66) solutos não iônicos em micelas formadas por $S D S$.

Dada a heterogeneidade tanto dos possíveis ambientes de solubilização quanto com relação à estrutura molecular e propriedades físico-química de cada soluto, é interessante que uma única equação de regressão linear seja adequada para correlacionar os dados. Entretanto essa metodologia deve ser aplicada depois do experimento. Além do mais, os valores dos parâmetros para moléculas complexas não são encontradas com facilidade e, em muitos casos, são de difícil obtenção experimental. Logo, a regressão linear pode somente ser usada em uma modalidade de previsão para o conjunto de parâmetros existentes. É nesse momento que a teoria pode trazer uma contribuição substancial. 


\section{Referências}

1. Draper, N.R.; Smith, H.; Applied Regression Analysis, Wiley, New York, 1981.

2. Hansch, C.; 1993, Acc. Chem. Res., 26,147.

3. Hammet, L. P.; 1937, J. Am. Chem. Soc., 59,96.

4. Kamlet, M. J.; Taft, R.W.; Abboud, J. L. M.; 1977, J. Am. Chem. Soc., 91,8325.

5. Kamlet, M. J.; Doherty, R. M.; Abraham, M. H; Taft, R.W.; 1988, Quant. Struct. Act. Relat., 7, 71.

6. Abraham, M. H.; 1993, Chem. Soc. Rev., 22, 73

7. Abraham, M. H.; 1995, Pure Appl. Chem., 65, 2503.

8. Abraham, M. H.; Whitting, G. S., Fuchs, R.; Chamber E. J.; 1990, J. Chem. Soc. Perkin Trans. 2, 291.

9. Taft, R.W.; Abraham, M. H.; Famini, G. R.; Doherty, R. M.; Abboud, J. M.; Kamlet, M. J.; 1985, J. Pharm.Sci., 74, 8, 807.

10. Abraham, M. H., Kamlet, M. J., Taft, R.W., 1992, J. Chem. Soc. Perkin Trans. 2, 923.

11. Abraham, M.H, Grellier, P.L., Abboud, J.L., Doherty, R.M., Taft, R.W., 1988, Can. J. Chem. 66, 2673.

12. Abraham, M. H.; Whiting, G. S., Doherty, R. M. e Shuely, W. J., 1990, J. Chem. Soc. Perkin Trans 2, 1451.

13. Abraham, M. H.; Whiting, G. S., Dorety, R. M., Shuely, W. J., 1991, J. Chromatogr., 587, 213.

14. Abraham, M. H.;Whiting, G. S., 1992, J. Chromatogr., 594, 229.

15. Taft, R. W.; Abraham, M. H.; Doherty, R. M.; Kamlet, M. J.; 1985,J. Am. Chem. Soc., 107, 3105 .

16. Kamlet, M. J.; Carr, P. W.; Taft, R. W.; Abraham, M. H.; 1981, J. Am. Chem Soc., 103, 6062 .

17. Abraham, M. H., McGowam, J. C., 1987, Chromatographia, 23, 243.

18. Quina, F. H.; Alonso, E. O.; Farah, J. P. S.; 1995, J. Phys. Chem.; 99, 11708.

19. Brokzijl, W.; Engberts, J. B. F. N.; 1993, Angew. Chem. Int. Ed. Engl., 32.1545. 


\section{Canítulo 3}

\section{Relação Linear Teórica de Energia liure de Soluatação}

No capítulo 2 , mostramos que a análise de regressão linear múltipla pode ser usada para estudar a transferência de um soluto e sua cavidade entre duas fases condensadas. A metodologia apresentada usa com sucesso um único conjunto de parâmetros do soluto, para correlacionar coeficientes de incorporação $\left(\mathrm{K}_{\mathrm{s}}\right)$ obtidos experimentalmente. Porém a aplicação deste modelo é restrito para solutos cujos parâmetros já tenham sido determinados experimentalmente. Logo uma metodologia mais geral, aplicável a qualquer soluto de interesse é substituir os parâmetros do soluto determinados experimentalmente, por parâmetros determinados unicamente a partir da estrutura molecular do soluto.

Neste capítulo, apresentaremos o formalismo matemático e o significado físico-químico dos parâmetros teóricos, usados como descritores na análise de regressão linear teórica de energia livre de solvatação(TLSER - Theoretical Linear Solvation Energy Relationship). 


\subsection{Correlação Linear Teórica de Energia Livre de Solvatação}

O principal fator, que limita a aplicabilidade das relaçōes lineares de energia livre de solvatação baseadas nos parâmetros do soluto de Abraham, é a falta dos valores destes parâmetros para solutos mais complexos, devido a dificuldades encontradas na determinação experimental. Em princípio, uma metodologia mais geral, aplicável a qualquer soluto de interesse, é substituir os parâmetros do soluto determinados experimentalmente ou empiricamente por parâmetros calculados unicamente a partir da estrutura molecular do soluto.

O crescente uso do computador nas três últimas décadas tem evidenciado o impacto do uso da tecnologia da informação nas ciências exatas; em particular, o cálculo de propriedades eletrônicas é hoje uma prática comum. Essa prática tem feito uso de diferentes nomes, isto é, química computacional, modelagem molecular, etc. Porém neste estudo referimo-nos apenas como "teoria". Identificamos primeiramente três truísmos onde a teoria deve ser aplicada: (a) a teoria deve ser aplicada a posteriori em situações onde exista ambigüidade na interpretação de resultados experimentais; (b) a teoria deve ser usada para melhorar a performance de um programa de pesquisa experimental; e, (c) a teoria deve ser usada para predizer propriedades que sejam difíceis, perigosas ou impossíveis de serem determinadas experimentalmente.

Nosso principal interesse está no uso de métodos de química quântica para calcular propriedades de estrutura eletrônica e, a partir das grandezas físicas calculadas, estimar coeficientes de incorporação de solutos não iônicos em micelas formadas por detergentes através do uso de análise de regressão linear múltipla.

Para este propósito, consideramos duas possibilidades, a fim de estabelecer uma relação direta com a equação (2.6). Primeiramente, pode-se tentar correlacionar propriedades estruturais e/ou eletrônicas com os parâmetros empíricos do soluto e, desse modo, obter uma fórmula ou receita para estimá-los a partir de cálculos de estrutura eletrônica. Tal metodologia foi explorada por Lewis ${ }^{1}$ e recentemente por Abraham ${ }^{2}$. Uma abordagem alternativa é manter o formalismo da relação linear de energia livre de solvatação, porém substituir os parâmetros empíricos por parâmetros calculados a partir da estrutura molecular do soluto. Neste segundo caso, Famini et. al. encontraram boas correlações no estudo de propriedades físico-químicas envolvendo equilíbrio, cinética e espectroscopia ${ }^{3-4}$. Nossa proposta é investigar a utilidade da abordagem de Famini para estimar coeficientes de incorporação de solutos não iônicos em micelas formadas por detergentes aniônicos, catiônicos e não iônicos.

\subsection{Modelo de Famini}

Uma metodologia aplicável a qualquer soluto de interesse permitiria, em princípio, prediçōes a priori. Neste intuito, Famini et. al. propuseram a relação linear teórica de energia livre de solvatação (TLSER-Theoretical Linear Solvation Energy Relationship). A metodologia adotada por Famini usa a filosofia e a estrutura de $L S E R s$, porém faz uso de parâmetros obtidos teoricamente, levando em conta três características: (a) deve correlacionar com os parâmetros de LSER; (b) a equação de regressão deve estimar coeficientes de correlação e desvios padrões com a mesma precisão de LSER; e, (c) deve ser de aplicação geral para interações que envolvem soluto-solvente. Os parâmetros derivados de métodos computacionais foram escolhidos para ter uma correspondência com os parâmetros empíricos de relação linear de energia livre de 
solvatação, os quais são descritos a seguir. O termo de volume para TLSER é descrito pelo volume molecular de van der Waals $\left(V_{m v}\right)$ dado em Ångstrons ao cubo. O termo relativo a dipolaridade/polarizabilidade é descrito através do índice de polarizabilidade $\left(\pi_{F}\right)$, o qual é obtido dividindo a polarizabilidade molecular $(\alpha)$ pelo volume molecular para produzir uma grandeza adimensional. Este termo define a facilidade com que a nuvem eletrônica é polarizável. Exatamente como na LSERs, o termo que descreve as interações por pontes de hidrogênio é separado em caráter ácido e básico. Porém, ao contrário da LSERS, a TLSER necessita de parâmetros individuais para essas contribuições, já que as ligações químicas são descritas por um termo covalente e outro eletrostático. A contribuição covalente para o caráter básico das interações de hidrogênio é descrita pela diferença de energia entre o orbital molecular ocupado de mais alta energia ( $\left.\varepsilon_{\text {помо }}\right)$ do soluto e o orbital desocupado de energia mais baixa $\left(\varepsilon_{l . u m o}\right)$ da água. Por razões práticas, o resultado da diferença entre as energias $\left(\varepsilon_{I \text { IOMo }}\right)$ do soluto e $\left(\varepsilon_{l . U M o}\right)$ da água é dividido por 100 para produzir valores da mesma ordem de grandeza do índice de polarizabilidade. A contribuição eletrostática para o caráter básico da ligação é descrita pela magnitude da maior carga formal negativa $(q$-) sobre o heteroátomo da molécula. Quanto maior o valor da carga formal negativa, maior deve ser a tendência a interagir ionicamente com os prótons das moléculas vizinhas.

A contribuição covalente para o caráter ácido é descrita pela diferença de energia entre o orbital molecular desocupado de energia mais baixa $\left(\varepsilon_{\text {I.UMo }}\right)$ do soluto e o orbital ocupado de mais alta energia $\left(\varepsilon_{\text {Iом⿰о }}\right)$ da água. Novamente por razões estéticas, o resultado da diferença de energia entre $\left(\varepsilon_{l . U m o}\right)$ do soluto e $\left(\varepsilon_{l l o m o}\right)$ da água é dividido por 100 para produzir valores da mesma ordem de grandeza do índice de polarizabilidade. Em analogia a basicidade, a contribuição eletrostática para a acidez é descrita pela maior carga formal positiva $\left(q_{+}\right)$sobre um átomo de hidrogênio contido na molécula e representa a porção da molécula do soluto com maior capacidade de interagir com a distribuição de cargas negativas das moléculas vizinhas. A partir da definição desses seis parâmetros teóricos, Famini propôs relação linear teórica de energia livre de solvatação, dada por:

$$
\log S S P=\mathrm{a}_{0}+\mathrm{a}_{1} q_{-}+\mathrm{a}_{2} q_{+}+\mathrm{a}_{3} \pi_{\mathrm{F}}+\mathrm{a}_{4} \varepsilon_{l / O M O}+\mathrm{a}_{5} \varepsilon_{l . U M O}+\mathrm{a}_{6}\left(\mathrm{~V}_{\mathrm{mv}} / 100\right)
$$

onde SSP representa a propriedade físico-química de interesse, sendo geralmente usado o logaritmo do valor da medida. Na Figura 3.1 mostramos um esquema representando o significado físico-químico dos parâmetros $\left|q_{-}\right|, q_{+}, \pi_{l}, \varepsilon_{l(\omega m)}, \varepsilon_{l, U M}$, e $V_{\mathrm{mv}}$, Em essência, no modelo de Famini a escolha do número apropriado de parâmetros a ser usado em um determinado estudo e a origem dos valores para esses parâmetros está baseado unicamente na intuição química do pesquisador, sendo que a função dos parâmetros escolhidos é espelhar as principais interações que envolvem o problema em estudo, especialmente em relação à magnitude e sinal dos coeficientes. A equação (3.1) tem sido aplicada para uma grande variedade de fenômenos físicos, químicos e biológicos. Na Tabela 3.1 mostramos algumas das propriedades investigadas usando TLSER e suas respectivas referências. Os parâmetros usados na TLSER apresentam uma boa correlação e interpretação físico-química satisfatória dos fenômenos estudados. Cabe aqui observar que quando comparações são feitas entre os resultados obtidos usando TLSER e LSERS, a interpretação físico-química é consistente com $L S E R$, porém as correlações de $L S E R S$ são em geral estatisticamente mais significativas. 

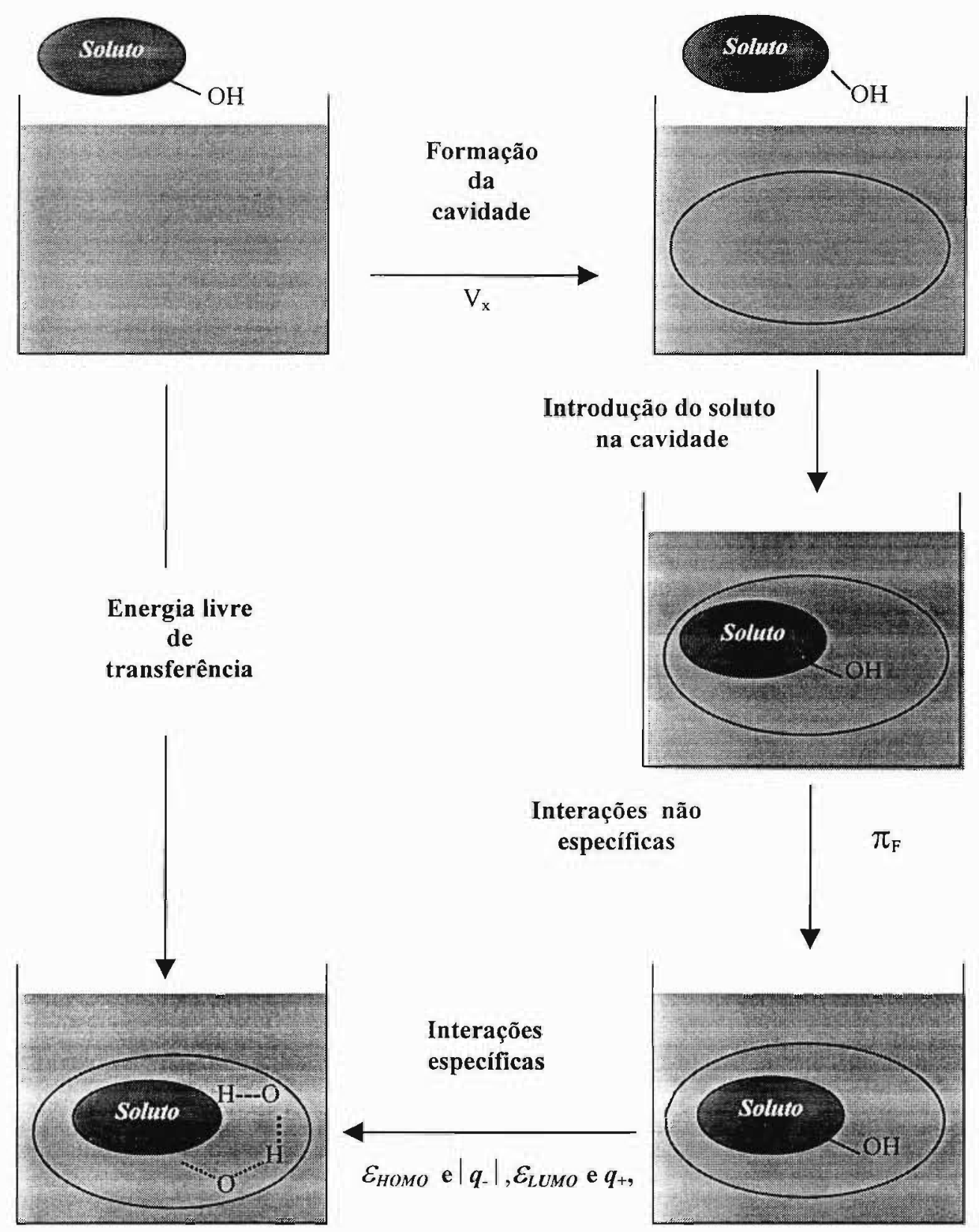

Figura 3.1 Significado físico-químico dos parâmetros teóricos do soluto inerentes a relação linear teórica de energia livre de solvatação (TLSER). 


\section{Tabela 3.1 Propriedades físico-químicas e biológicas}

estudadas usando TLSER.

\begin{tabular}{lc}
\hline \hline Propriedades correlacionadas & Referência \\
\hline \hline Könemann poluentes industriais & 3 \\
Adsorção em carvão & 3 \\
Coeficiente de partição octanol-água & 5 \\
Concentração mínima de retenção & 6 \\
Solubilidade em $\mathrm{CO}_{2}$ & $7-9$ \\
Acidez em fase gasosa & 10 \\
Polarizabilidade & 11 \\
Parâmetros de Hammet & 12 \\
Formação de radicais livres & 13 \\
Metabolismo de ácidos benzóicos & 14 \\
\hline
\end{tabular}

Conseqüentemente, os parâmetros teóricos podem ser usados da mesma forma que os parâmetros obtidos experimentalmente. Em particular, cálculos de propriedades eletrônicas são largamente utilizados e requerem somente o cálculo da funçāo de onda, a partir da qual poderemos calcular uma variedade de observáveis com potencial para serem empregados como descritores. No próximo capítulo apresentaremos as bases conceituais e o formalismo $H F$ semiempírico usado neste estudo para calcular parâmetros teóricos de energia livre.

\section{Referências}

1. Lewis, D. F. V.; 1987, J. Comput. Chem.; 8, 1084.

2. Platts, J. A.; Butina, D.; Abraham, M. H.;Hersey, A.; 1999, J. Chem. Inf.Comput. Sci. 39, 835.

3. Famini, G. R.; Pensky, C. A.; Wilson, L. Y.; 1992, J. Phy. Org. Chem.; 5, 395.

4. Cramer, I. J.; Famini, G. R; Lowey, A. H.; 1993, Acc. Chem. Res.; 26, 599.

5. Wilson, L.Y.; Famini, G. R.; 1991, J. Med. Chem.; 5,1668.

6. Famini, G. R.; Kassel, R. J.; King, J. W.; Wilson, L. Y.; 1991, Quant. Struct-Act. Relat., 10, 334.

7. Politzer, P.; Murray, J-S.; Lane, P.; Brinck, T.; 1993, J. Phys. Chem., 97, 729.

8. Murray, J-S,.; Lane, P.; Brinck, T.; Politzer, P.; 1993, J. Phys. Chem., 97, 5144.

9. Politzer, P.; Murray, J-S.; Concha, M. C.; Brinck,T.; 1993, J. Mol. Struct. (THEOCHEM), 100, 107.

10. Famini, G. R.; Marques, B. C.; Wilson, L. Y.; 1993, J. Chem. Perkin Trans. 2, 773.

11 Brinck, T.; Murray, J-S.; Politzer, P.; 1993, J. Chem Phys., 98, 4305.

12. Murray, J-S.; Brinck, T.; Politzer, P.; 1992, J. Molec. Struct. (THEOCHEM),87, 271.

13. Paulina-Blumenfeld, M.; Hansz, M.; Hikichi, N. Sooppani, A. O. M.; 1992, Free Radicals Res. Commun., 16, 207.

14. Ghouri, F.Y.; Blackedge, C. A. Glen, R. C.; Sweatman, B. C.; Lindon, J. C.; Beddell, C. R.;Wilson, I. D.; Nicholson, J. K.; 1992, Biochem. Pharmacol., 44, 1935. 


\section{Capítulo 4}

\section{Formalismo Matemático Hartree-Fock-Roothaan}

Neste capítulo, apresentamos o formalismo matemático do método de Hartree-FockRoothaan em sua versão semi-empírica. Este formalismo está implementado nos programas MOPAC-93 e Amsol-5.4, os quais foram empregados para calcular os parâmetros teóricos usados como descritores nas relações lineares teórica de energia livre de solvatação.. 


\subsection{Cálculos de Estrutura Eletrônica}

Os métodos de cálculos de estrutura eletrônica fazem uso de princípios e leis da mecânica quântica como base fundamental para seu cômputo. Quanticamente falando, a energia de uma molécula e todas as suas propriedades podem ser determinadas através da solução da equação de Schrödinger. Poucos anos após a formulação da equação de Schrödinger, Hylleraas ${ }^{1}$ conseguiu resolver o átomo de hélio com precisão impressionante e foi historicamente o primeiro suporte para a crença, hoje generalizada, da validação da equação de Schrödinger. Através do uso de coordenadas inter-eletrônicas, Hylleeraas introduziu explicitamente a correlação do movimento dos elétrons. Apesar do sucesso desta formulação, cedo tornou-se aparente que o uso de coordenadas inter-eletrônicas tornava-se impraticável para sistemas com mais de cinco ou seis elétrons. Hoje é reconhecido que o uso de coordenadas inter-eletrônicas requer uma sofísticação computacional grande e deve ser reservada para sistemas muito pequenos.

Como a teoria deve satisfazer o requisito de ampla aplicação e este critério não é satisfeito pelo método de Hylleraas, a quase totalidade dos métodos ab-initio e semiempíricos utilizados presentemente tem como ponto de partida o método desenvolvido independentemente por Hartree ${ }^{2}$ e Fock ${ }^{3}$, sistematizado por Slater ${ }^{4}$, que mostrou sua característica variacional, e Roothaan ${ }^{5}$, que deu ao método sua estrutura atual. De certa forma, o método $H F$ é uma continuação das idéias de Bohr ${ }^{6}$ e de seu modelo de partículas independentes, onde os processos são descritos a nível de 1-partícula, não podendo assim descrever efeitos de muitas partículas (efeitos de correlação eletrônica). Adicionalmente, cada partícula no modelo de $H F$ move-se num campo médio provocado pelos outros elétrons, isto é, o movimento não é correlacionado pelo potencial coulombiano.

Atualmente, a obtenção da solução exata da equação de Schrödinger é possível somente para moléculas simples, como por exemplo $\mathrm{H}_{2}$ e $\mathrm{H}_{2}{ }^{+}$, ou seja, a química teórica está fundamentada no tratamento aproximado da teoria quântica e, como consequência, os métodos de cálculos de estrutura eletrônica caracterizam-se pelas aproximações matemáticas usadas para obter a solução da equação de Schrödinger. Diante disto, é compreensível que haja um grande número de teóricos que dirigem seus esforços na tentativa de resolver a equação de Schrödinger com um grau cada vez maior de precisão numérica. Nas últimas três décadas avanços consideráveis foram obtidos neste sentido.

\subsection{Teoria do Orbital Molecular}

A primeira aproximação que consideraremos é a aproximação do orbital molecular para ligações químicas. Nesta aproximação um elétron ocupa um orbital, isto é, uma região do espaço que se espalha por toda a molécula. Uma prática comum é construir orbitais moleculares através de uma combinação linear de orbitais atômicos (LCAO Linear Combination of Atomic Orbitals) dos constituintes da molécula.

A aproximação $L C A O$ descreve a estrutura eletrônica, a forma e a energia de uma molécula, considerando a interpretação da ligação química em termos de acúmulo de densidade de carga na região inter-nuclear. O ponto central dessa aproximação é construir orbitais moleculares, possuindo simetria apropriada, para que haja um sobreposição entre os diferentes orbitais atômicos tipo hidrogenóides $(s, p, d, f, .$.$) . Um orbital molecular é$ então construído a partir da combinação linear de um conjunto de funções matemáticas 
pré-definidas conhecidas como funções base. Podemos então expandir os orbitais moleculares com uma combinação linear de orbitais atômicos $\left\{\varphi_{\mu}\right\}$.

$$
\psi_{i}=\sum \mathcal{c}_{\mu i} \varphi_{\mu}
$$

onde os coeficientes $\mathrm{C}_{\mu \mathrm{i}}$ são conhecidos como coeficientes de expansão do orbital molecular. Se o conjunto de funções base $\left\{\varphi_{\mu}\right\}$ for completo, teremos uma solução exata. Na prática, entretanto, o conjunto é finito; com isto, usa-se uma aproximação de base finita e a solução da equação de Schrödinger não é exata, mas sim uma solução aproximada. Quanto mais completo for o conjunto das funções base, melhor será o valor obtido para a energia do molécula. Na literatura existe uma variedade de funções base compostas de diferentes funções atômicas, sendo duas as de uso mais corrente: as funções de Slater ${ }^{7}$ (STO - Slater-Type Orbital) e as gaussianas ${ }^{8}$ (GTO - Gaussian - Type Orbital). As funções de Slater são funções analíticas com parâmetros que são ajustados às soluções numéricas da equação de Schrödinger, e possuem a seguinte forma,

$$
\psi_{(r, \theta, \phi)}^{S T O}=N r^{n^{*}-1} e^{-\xi r} Y_{l m}
$$

onde $\xi$ é o exponencial orbital, dado por $\xi=(Z-\sigma) / n^{*}, \sigma$ é a constante de blindagem, $n^{*}$ está relacionado ao número quântico principal, $N$ é o fator de normalização e $Y_{l m}$ descreve os harmônicos esféricos. As funções de base GTO são formadas por funções gaussianas e, em geral, são utilizadas em substituição à base de Slater, com a qual é difícil encontrar uma forma analitica para integrais multicentradas.

$$
\psi_{(r)}^{G T O}=N x^{k} y^{\prime} z^{m} \exp \left(-\alpha r^{2}\right)
$$

Os orbitais atômicos expressos em funções gaussianas são a soma de várias gaussianas e, por isso, para descrever convenientemente um fenômeno físico-químico, precisa-se sempre de um número maior de gaussianas do que de funções de Slater.

$\mathrm{Na}$ aproximação de base mínima, ou seja, quando se toma o conjunto de funções de base igual ao número de orbitais atômicos ocupados, é mais conveniente a escolha das funções de Slater, apesar do processo de cálculo das integrais multicentradas ser. mais simples quando efetuado com funções de base gaussianas.

\subsection{A Equação de Schrödinger Independente do Tempo}

A segunda aproximação que consideraremos está relacionada com o ponto de partida para qualquer estudo de estrutura eletrônica, isto é, a procura da solução aproximada da equação de Schrödinger para um sistema poliatômico.

$$
\left[\frac{-h^{2}}{8 \pi^{2} m} \nabla^{2}+V(r, t)\right] \Psi(r, t)=\frac{i h}{2 \pi} \frac{\partial \Psi(r, t)}{\partial t}
$$

Na equação de Schrödinger, $\Psi(r, t)$ é a função de onda do sistema em estudo, $m$ a massa da partícula, $h$ a constante de Planck e $V(r, t)$ o potencial no qual os elétrons se movem. Para 
uma molécula $\Psi(r, t)$ será uma função das coordenadas de todas as partículas que compõem o sistema. Se o potencial $V(r, t)$ não é função do tempo, isto é, pode ser descrito apenas como função da posição $V(r)$, a equação de Schrödinger pode ser simplificada usando a técnica matemática de separação de variáveis. Então, podemos escrever a função de onda $\Psi(r, t)$ como um produto das partes espacial e temporal da função ou seja:

$$
\Psi(r, t)=\Psi(r) \Phi(t)
$$

Substituindo a equação (4.5) na equação (4.4) obteremos duas equações, uma que depende apenas da posição das partículas e é independente do tempo e outra que é função do tempo e independente da posição. Para os problemas de interesse físico-químico, a separação de variáveis é válida e o problema passa a ser focalizado na solução da equação de Schrödinger independente do tempo, sendo $E$ a energia do sistema e $H$ o operador hamiltoniano não relativístico.

$$
H \Psi(r)=E \Psi(r)
$$

Para um sistema molecular, $\Psi(r)$ na equação (4.6) é uma função da posição dos elétrons e núcleos que compõe a molécula, os quais designaremos por $r$ e $R$ respectivamente. Os símbolos $r$ e $R$ representam o conjunto de coordenadas que descrevem a posição de cada partícula do sistema, onde

$\mathrm{e}$

$$
H=\frac{-h^{2}}{8 \pi^{2} m} \nabla^{2}+V(r)
$$

$$
V(r)=\left(\frac{1}{4 \pi \varepsilon_{0}}\right) \sum_{i} \sum_{j} \frac{e_{j} e_{k}}{\nabla r_{i k}}
$$

Logo a equação (4.4) poderá ser escrita na forma da equação (4.6). A equação (4.6) é uma equação de autovalor do operador hamiltoniano $H$, sendo conhecida como a equação de Schrödinger independente do tempo, em oposição a equação (4.4) dependente do tempo. Em nosso estudo, estamos interessados em resolver a equação de Schrödinger independente do tempo, a qual possibilita obter, entre todos os estados possíveis do sistema, aqueles que são estacionários.

\subsection{Aproximação de Born-Oppenheimer}

A terceira aproximação a ser considerada é a aproximação de Born-Oppenheimer. Do ponto de vista de um sistema de coordenadas moleculares, podemos escrever o hamiltoniano (em unidades atômicas) para um sistema de $N$ elétrons e $M$ núcleos como segue:

$$
H=-\sum_{i=1}^{N} \frac{1}{2} \nabla_{i}^{2}-\sum_{A=1}^{M} \frac{1}{2 M_{A}} \nabla_{A}^{2}-\sum_{i=1}^{N} \sum_{A=1}^{M} \frac{Z_{A}}{r_{i A}}+\sum_{i=1}^{N} \sum_{\substack{j=1 \\ j>1}}^{N} \frac{1}{r_{i j}}+\sum_{A=1}^{M} \sum_{\substack{B=1 \\ B>A}}^{M} \frac{Z_{A} Z_{B}}{R_{A B}}
$$


onde $M_{A}$ é a razão entre a massa do núcleo $A$ e a massa do elétron, $Z_{A}$ é o número atômico do núcleo $A$. O primeiro e segundo termos do hamiltoniano se referem às energias cinéticas dos elétrons e núcleos, respectivamente, o terceiro termo diz respeito à atração coulombiana entre elétrons e prótons e o quarto e quinto termos dizem respeito à repulsão mútua entre elétrons e prótons, respectivamente.

Uma vez que a massa do núcleo é da ordem de $10^{4}$ vezes maior que a massa do elétron, é razoável considerarmos o movimento do núcleo como sendo lento em relação ao movimento do elétron. Logo, uma boa aproximação consiste em considerar os elétrons da molécula se movendo em torno de núcleos fixos. Esta é a aproximação de BornOppenheimer, cuja função de onda total tem a seguinte forma:

$$
\Psi(r)=\Psi\left(\left\{r_{i}\right\} ;\left\{R_{A}\right\}\right)=\Psi_{e l e}\left(\left\{r_{i}\right\} ;\left\{R_{A}\right\}\right) \Psi_{n u c}\left(\left\{R_{A}\right\}\right)
$$

Sendo a função de onda total do sistema expressa pela equação (4.9), podemos desprezar o segundo termo da equação (4.9) e o último termo pode ser considerado como uma constante. Os termos remanescentes constituem o chamado hamiltoniano eletrônico, isto é, um hamiltoniano que descreve o movimento de $N$ elétrons em torno de $M$ cargas pontuais,

$$
H_{e l e}=-\sum_{i=1}^{N} \frac{1}{2} \nabla_{i}^{2}-\sum_{i=1}^{N} \sum_{A=1}^{M} \frac{Z_{A}}{r_{i A}}+\sum_{\substack{i=1 \\ j=1 \\ j>i}} \frac{1}{r_{i j}}
$$

Portanto, temos que resolver a seguinte equação de autovalor

onde,

$$
H_{\text {ele }} \Psi_{\text {ele }}(r)=E_{\text {ele }} \Psi_{\text {ele }}(r)
$$

$$
\Psi_{\text {ele }}=\Psi_{\text {ele }}\left(\left\{r_{i}\right\} ;\left\{R_{A}\right\}\right)
$$

ou seja, a função de onda eletrônica, solução da equação de Schrödinger para o hamiltoniano eletrônico, depende explicitamente das coordenadas eletrônicas e parametricamente das coordenadas dos núcleos. Desta forma, podemos obter a energia total do sistema, incluindo a constante de repulsão nuclear,

$$
E_{\text {tot }}=E_{\text {ele }}+\sum_{A=1}^{M} \sum_{B=1}^{M} \frac{Z_{A} Z_{B}}{R_{A B}}
$$

Para resolver a parte nuclear, basta considerarmos o núcleo se movendo em um campo médio de $N$ elétrons, ou seja,

$$
\begin{aligned}
& H_{n u c}=-\sum_{A=1}^{M} \frac{1}{2 M_{A}} \nabla_{A}^{2}+\sum_{A=1}^{M} \sum_{B>A}^{M} \frac{Z_{A} Z_{B}}{R_{A B}}+\left\langle-\sum_{i=1}^{N} \frac{1}{2} \nabla_{i}^{2}-\sum_{i=1}^{N} \sum_{A=1}^{M} \frac{Z_{A}}{r_{i A}}+\sum_{i=1}^{N} \sum_{j>i}^{N} \frac{1}{r_{i j}}\right\rangle \\
& H_{n u c}=-\sum_{A=1}^{M} \frac{1}{2 M_{A}} \nabla_{A}^{2}+\sum_{A=1}^{M} \sum_{B>A}^{M} \frac{Z_{A} Z_{B}}{R_{A B}}+E_{\text {ele }}\left(\left\{R_{A}\right\}\right)=-\sum_{A=1}^{M} \frac{1}{2 r_{A}} \nabla_{A}^{2}+E_{\text {rot }}\left(\left\{R_{A}\right\}\right)
\end{aligned}
$$


Portanto, a energia total funciona como um potencial para o movimento nuclear ou seja,

$$
H_{n u c} \Psi(r)=E_{n u c} \Psi(r)
$$

onde,

$$
\Psi_{n u c}=\Psi_{n u c}\left(\left\{R_{A}\right\}\right)
$$

A equação (4.15) pode ser usada para descrever as vibrações, rotações e translações da molécula. Devido a natureza e objetivo do nosso estudo, será suficiente resolver a equação de Schrödinger para os estados eletrônicos estacionários da molécula.

\subsection{Aproximação de Hartree-Fock-Roothaan}

A quarta aproximação que consideraremos diz respeito à maneira de resolver a equação de Schrödinger independente do tempo para um sistema poliatômico. Já que não é possível resolver a equação de Schrödinger para um sistema de $n$-elétrons, o método de $H F$ propõe a substituir este problema pelo problema mais simples de se resolver $n$-equações de 1-elétron. Desta forma, ao método de $H F$ está associado um modelo de 1-elétron.

$$
H \Psi(1,2, \ldots, N)=E \Psi(1,2, \ldots, N)
$$

onde

$$
H=\sum_{i=1}^{N} h_{i}+\sum_{i<j}^{N} \sum^{N} \frac{1}{r_{i j}}
$$

sendo

$$
h_{i}=-\frac{1}{2} \nabla_{i}^{2}-\sum_{A=1}^{A I} \frac{Z_{A}}{r_{i A}}
$$

dependente da coordenada de um único elétron. Nas equações acima, $N$ é o número total de elétrons na molécula, $M$ o número de átomos e $r_{i j}$ é a distância entre o elétron $i$ e o elétron $j, Z_{A}$ é a carga nuclear do átomo $A, r_{i A}$ é a distância entre o elétron $i$ e o núcleo $A$. O operador $\Sigma \Sigma\left(1 / \mathrm{r}_{\mathrm{ij}}\right)$ corresponde à energia potencial devido à interação do elétron $i$ com o elétron $j$. $\mathrm{O}$ operador Laplaciano $\left(-1 / 2 \nabla_{i}^{2}\right)$ corresponde à energia cinética do elétron i. $\mathrm{O}$ operador $\sum Z_{\mathrm{A}} / \mathrm{r}_{\mathrm{iA}}$ dará a energia potencial de interação entre o elétron $i$ e os $M$ núcleos. $\Psi(1,2, \ldots, N)$ é a função multieletrônica, $\operatorname{com}(1,2, \ldots, N)$ indicando as respectivas coordenadas dos $N$ elétrons. A função de onda deve obedecer à lei da indistinguibilidade e da antissimetria quanto à permutação de duas partículas, o que leva ao princípio de exclusão de Pauli. Estas restrições estarão satisfeitas ao se expressar à função de onda multieletrônica através de determinante de Slater dos orbitais moleculares de 1-elétron $\psi$ $(j)$

$$
\Psi(1,2, \ldots, N)=\left|\psi_{1}(1) \psi_{2}(2) \psi_{3}(3) \ldots \ldots \psi_{n}(N)\right|
$$


As funções $\psi_{i}(j)$ são funções de 1-elétron obtidas pelo método de $H F$ da solução de um conjunto de equações de autovalores

$$
H(i) \psi_{i}(i)=\varepsilon_{i} \psi_{i}(i)
$$

onde $H(i)$ é a hamiltoniana efetiva para um elétron no orbital $\psi_{i}(i)$ e a quantidade $\varepsilon_{1}$ é a energia do orbital de um elétron. Este hamiltoniano depende das posições relativas dos outros elétrons através de seu campo coulômbico médio e do campo de troca (exchange)

$$
H_{e f f}(i)=h_{i}+\sum_{l}\left(2 J_{l}-K_{l}\right)
$$

onde $J_{l}$ e $K_{l}$ são, respectivamente, os operadores coulômbicos e de troca, definidos em termos de $\psi_{k}(i)$

$$
\begin{array}{r}
J_{l} \psi_{K^{K}}(i)=\left\{\int \psi_{l}(j)^{*} \frac{1}{r_{i j}} \psi_{l}(j) d \tau_{j}\right\} \psi_{K^{\prime}}(i) \\
K_{l} \psi_{K^{\prime}}(i)=\left\{\int \psi_{l}(j)^{*} \frac{1}{r_{i j}} \psi_{K^{\prime}}(j) d \tau_{j}\right\} \psi_{l}(i)
\end{array}
$$

A determinação de $\psi_{k}(i)$ implica no conhecimento das outras autofunções e, assim, este sistema de equações deve ser resolvido simultaneamente para todos os orbitais moleculares, o que é realizado por métodos autoconsistentes (SCF - Self Consistent Field). Os orbitais $\psi_{i}(i)$ são, então, autofunções do operador $H(i)$ e os autovalores $\varepsilon_{i}$ as energias dos orbitais de um elétron. No método $H F$, a energia eletrônica total de um sistema poliatômico é dada pela equação

$$
E=2 \sum_{j}\left\langle i\left|h^{N}\right| i\right\rangle+\sum_{i j}\left(2 J_{i j}-K_{i j}\right)
$$

onde

$$
\begin{gathered}
\left\langle i\left|h^{N}\right| i\right\rangle=\int \psi_{i}(1) h^{N} \psi_{i}(1) d \tau_{1}=H \stackrel{N}{i i} \\
h^{N}=-\frac{1}{2} \nabla_{1}-\sum_{A=1}^{N^{\prime}} \frac{Z_{A}}{r_{1 A}} \\
J_{i j}=\langle i i \mid j j\rangle=\int \psi_{i}(1) J_{i} \psi_{i}(1) d \tau_{1}
\end{gathered}
$$




$$
K_{i j}=\langle i j / i j\rangle=\int \psi_{i}(1) K_{j} \psi_{i}(1) d \tau_{1}
$$

Os símbolos $J_{i j}$ e $K_{i j}$ denotam respectivamente a integral de repulsão coulômbica e a integral de exchange, onde o bra se refere ao elétron 1 e o ket ao elétron 2.

Os orbitais moleculares $\psi_{i}$ são expandidos em termos de uma combinação linear de orbitais atômicos $\left\{\varphi_{\mu}\right\}$, descritos pela equação (4.1) onde $\mu$ indica o orbital atômico e $i$ o estado molecular. No processo variacional os coeficientes moleculares $c_{\mu i}$ são variados, obtendo-se as equações de Hartree-Fock-Rootham (HFR) e a seguinte expressão para a energia eletrônica

$$
E_{H F}=\frac{1}{2} \sum_{\mu \nu} P_{\mu v}\left(H_{\mu v}^{N}+F_{\mu v}^{(0)}\right)
$$

onde

$$
H_{\mu \nu}^{N}=\left\langle\varphi_{\mu}(1)\left|-\frac{1}{2} \nabla_{1}^{2}-\sum_{A=1}^{N} \frac{Z_{A}}{r_{1 A}}\right| \varphi_{v}(1)>\right.
$$

$\mathrm{e}$

$$
\begin{gathered}
F_{\mu \nu^{\prime}}^{(1)}=H_{\mu \nu^{\prime}}^{\lambda}+\sum_{\lambda \sigma} P_{\lambda \sigma}\left[<\mu \nu\left|\lambda \sigma>-\frac{1}{2}<\mu \sigma\right| \lambda \nu>\right] \\
P_{\lambda \sigma}=2 \sum_{i}^{o c c} C_{\lambda i} C_{\sigma i}^{*}
\end{gathered}
$$

a soma sendo feita sobre os estados moleculares ocupados, onde símbolo $\langle\lambda \mu \mid \lambda \sigma\rangle$ se refere-se a uma integral tal que $\mu \nu$ está associado ao elétron (1) e $\lambda \sigma$ ao elétron (2). Considerando como verdadeira a energia do sistema obtida experimentalmente, $\left(E_{\text {exp }}\right)$, veremos que esta energia difere sempre da obtida por métodos $S C F$. A energia total do sistema será considerado como a soma da energia computada usando a aproximação $H F$, mais as correções de correlação eletrônica e de termos relativísticos.

$$
E_{\text {exp }}=E_{c o r r}+E_{H F}+E_{r e l}
$$

Desta forma, podemos obter informação da qualidade da aproximação $H F$. Já que cálculos que envolvam $E_{r e l}$ ainda não são factiveis para sistemas poliatômicos, neste estudo, não levaremos em conta a $E_{c o r r}$ e $E_{\text {rel }}$.

\subsection{Métodos SCF -LCAO Semi-Empíricos}

Um dos objetivos da química é explicar a maneira pela qual átomos se combinam para formar moléculas e estimar taxas de reações. Porém, do ponto de vista teórico tais informações dependem da possibilidade de calcular a energia de uma coleção de átomos, em função de seu arranjo geométrico espacial. Se quisermos obter informações dessa natureza que sejam úteis do ponto de vista do químico, duas condições devem ser satisfeitas. Os cálculos devem ser precisos o suficiente para serem quimicamente 
significantes. Segundo, os cálculos devem ter custo inferior ao experimento equivalente, sendo que o valor desse custo dependerá do tipo de precisão desejada.

Hoje temos dois métodos para calcular o comportamento de um sistema poliatômico usando o método SCF- $L C A O$ : Os métodos ab-initio, os quais não necessitam de parâmetros empíricos, ou seja, são calculados a partir de primeiros princípios; e os métodos semi-empíricos, que empregam parâmetros determinados experimentalmente. $\mathrm{O}$ método de $(H F R)$ é um método $a b$ initio. Existe, porém, uma grande dificuldade computacional nos cálculos de algumas integrais, principalmente no cálculo das integrais multicentradas de 2- elétrons, o que reduz a aplicação do método a sistemas com um número pequeno de átomos. Em um esquema $S C F-L C A O$ semi-empírico, algumas dessas integrais são estimadas a partir de referências empíricas, podendo-se negligenciar certos elementos matriciais e fazer estimativas em outros. Nos métodos semi-empíricos têm-se duas etapas a cumprir. A primeira consiste em implementar uma aproximação que permita desprezar algumas partes das equações de $H F R$ e a segunda consiste em estimar as partes restantes. Faz-se, portanto, uma simplificação formal de um método $a b$ initio e adiciona-se informações baseadas em dados experimentais. Os métodos semi-empíricos existem para o tratamento de sistemas poliatômicos e são caracterizados através das aproximações que lhe são próprias. Os resultados obtidos, via métodos semi-empíricos, podem ser comparados com os provenientes de cálculos ab initio e com os valores experimentais, considerados como exatos. Neste estudo, faremos uso de métodos semi-empíricos nos quais se faz aproximação dos elétrons de valência.

\subsubsection{Aproximação dos Elétrons de Valência}

Experimentos indicam que os elétrons das camadas internas são relativamente inertes na formação de ligações químicas. Assim, poderemos expressar a função molecular eletrônica $\psi$ como produto antissimétrico das funções de caroço $\psi_{\text {core }}$, que representam os elétrons das camadas internas, e $\psi_{v a l}$ que descrevem os elétrons da camada de valência, sendo $\mathfrak{R}$ um operador antissimetrizador.

$$
\psi=\mathfrak{R}\left(|\psi\rangle_{\text {core },}|\psi\rangle_{\text {val }}\right)
$$

Os elétrons das camadas internas e o núcleo representam uma fonte de potencial eletrostático para os elétrons da camada de valência. Assim, desta forma, as cargas eletrônicas dos elétrons das camadas internas são englobadas à carga nuclear $Z_{A}$, formando uma carga efetiva de caroço $Z_{A}^{\prime}$. O operador de 1 -elétron $h^{\circ}(l)$ é reescrito como (veja equação (4.19))

$$
h^{\circ}(1)=-\frac{1}{2} \nabla_{i}^{2}-\sum_{A=1}^{N} \frac{Z_{A}^{\prime}}{r_{1 . A}}
$$

Este operador é denominado hamiltoniano de caroço (core-hamiltonian). Ele representa o operador de energia total para um elétron se movendo num campo de $N$ núcleos atômicos com carga nuclear efetiva $Z_{A}^{\prime}$. Esta aproximação reduz o número de elétrons a ser tratado, 
Porém o tratamento dos elétrons restantes ainda demanda um grande esforço computacional, sendo portanto necessário outras aproximações.

\subsubsection{Aproximação ZDO (Zero Differential Overlap)}

Este método foi introduzido independentemente por Pariser e Parr e por Pople ${ }^{9-10}$ onde são implementadas simplificações nas equações de HFR. As aproximações implementadas neste método são:

(a) o produto de dois orbitais atômicos é identicamente nulo a menos que estes orbitais se refiram a orbitais de mesmo átomo, isto é, $\mu=v$, ou seja, todas as integrais de 2-elétrons provenientes da sobreposição de dois orbitais atômicos centrados em diferentes átomos não são calculados.

$$
\Phi_{\mu_{A}} \Phi_{\nu B}=\delta_{\mu_{A} v_{B}} \delta \Phi_{\mu_{A}} \Phi_{v_{B}}
$$

onde $\mu$ ev especificam os orbitais atômicos e $A$ e $B$ os átomos em que os orbitais estão centrados.

(b) decorrentes da aproximação (a) temos que

$$
\left(\mu_{A} v_{B} \mid \lambda_{C} \sigma_{D}\right)=\left(\mu_{A} \mu_{A} \mid \lambda_{C} \lambda_{C}\right) \delta_{\mu_{A} v^{*}} \delta_{\lambda B C D}
$$

Com isto, a teoria considera somente integrais de um e dois centros, ou seja as integrais de três e quatro centros não são consideradas, pois não há rotação que converta uma integral de 2 -elétrons em um conjunto de integrais envolvendo três e quatro centros. A invariância rotacional não é comprometida pela aproximação.

(c) os elementos diagonais da matriz de caroço são fracionadas em um termo atômico $U \mu_{A} v_{A}$ e em outro constituído pela soma de termos de dois centros sobre os átomos da molécula .

\subsubsection{Elementos de Matriz HFR}

Devido a aproximação $Z D O$, os elementos da matriz de Fock dada pela equação (4.37) são simplificados. Nos elementos de $H \mu \nu$ que representam a atração do caroço efetivo $Z_{A}$ pelos elétrons da camada de valência, termos com $\mu \neq v$ cancelam-se. Os termos que representam a atração dos elétrons $\mu_{\mathrm{A}}$ pelo seu caroço $A$ são descritos por

$$
U_{\mu \mu}^{A}=-Z_{A}^{\prime} \int \frac{\Phi_{v A}(i) \Phi_{\mu-1}(i)}{r_{A v}} d \tau_{i}+\frac{1}{2} \int \Phi_{\mu_{A}}(i) \nabla_{i}^{2} \Phi_{v_{A}}(i) d \tau_{i}
$$

e, portanto,

$$
H_{\mu \mu}^{A}=U_{\mu \mu}^{A}-\sum_{B \neq A} V_{\mu \mu}^{B}
$$


A segunda parcela da equação (4.41) expressa a atração do elétron $\mu$ pelos demais núcleos atômicos Caso $\mu \neq v$, a atração elétron-caroço é cancelada, restando apenas os termos da energia cinética, que usualmente são tratados em termos de parâmetros empíricos $\beta_{\mu \nu}$ proporcionais a integral de sobreposição.

$$
H_{\mu \nu} \approx S_{\mu \nu} B_{\mu \nu}
$$

Os termos da matriz de Fock para dois elétrons são dados por,

$$
<\mu \nu\left|\lambda \sigma>=-\frac{1}{2}<\mu \sigma\right| \lambda \mu>
$$

que no aproximação $Z D O$ se modificam da seguinte forma:

(a) se $\mu=v e \lambda=\sigma$, o segundo termo da equação (4.41) é zero, restando somente as integrais

$$
<\mu \mu \mid \lambda \lambda>
$$

(b) se $\mu=\sigma e \nu=\lambda$, a primeira parcela da equação (4.41) será nula, restando as integrais

$$
\frac{1}{2}<\mu \mu \mid \mu \mu>
$$

(c) se $\mu=v=\lambda=\sigma$, haverá contribuição das duas parcelas da equação (4.41) e portanto as integrais:

$$
\frac{1}{2}<\mu \mu \mid \lambda \lambda>
$$

Desta forma, a aplicação da aproximação $Z D O$ reduz em muito o trabalho computacional, sendo que a matriz de Fock será representada por

$$
F_{\mu \mu}=U_{\mu \mu}^{A}-\sum_{B \neq A} V_{\mu \mu}^{B}+\sum_{\lambda} P_{\lambda \lambda}\langle\mu \mu \mid \lambda \lambda\rangle-\frac{1}{2} \sum_{\mu} P_{\mu \mu}\langle\mu \mu \mid \mu \mu\rangle
$$

$\mathrm{e}$

$$
F_{\mu \nu}=H_{\mu v}-\frac{1}{2} P_{\mu \nu}\langle\mu v \mid \mu v\rangle
$$

Dentro destas aproximações a matriz secular é dada por:

$$
C_{\mu \nu}\left|F_{\mu v}-\varepsilon S_{\mu \nu}\right| C_{\mu v}=0
$$

onde a condição de normalização estabelece que, utilizando a equação (4.1) 


$$
\sum_{\mu} \sum_{\nu} C_{\mu}^{i} C_{\nu}^{i} S_{\mu \nu}^{i}=1
$$

A energia do orbital molecular $k$ será,

$$
\varepsilon^{k}=\sum_{\mu} \sum_{\nu} C_{\mu}^{k} C_{\mu}^{k} F_{\mu \nu}
$$

e a energia total do sistema

$$
E_{H F}=\sum_{A<B} \frac{Z_{A}^{\prime} Z_{B}^{\prime}}{r_{A B}}+\sum_{\mu} \sum_{v} P_{\mu \nu} \frac{1}{2}\left(H_{\mu \nu}+F_{\mu \nu}\right)
$$

As aproximações efetuadas em $Z D O$ estão presentes em todos os métodos semiempíricos $L C A O$. A equação (4.52) representa a energia envolvida quando átomos ionizados e elétrons de valência são combinados para formar a molécula. Porém, a grandeza física mais usada em química é o calor de formação da molécula, o qual é definido como,

$$
\Delta H_{f}=\sum_{A} \Delta H_{f}^{A}-D
$$

onde o primeiro termo representa a soma dos calores de formação de todos os átomos que compõem a molécula e $D$ é a energia de dissociação (freqüentemente chamada de energia de atomização ou energia de ligação). O calor de formação reflete a estabilidade da molécula em relação aos substratos que leva a sua formação. Quanto maior o valor absoluto do calor de formação maior a estabilidade da molécula.

O método de $H F$ não prediz corretamente o calor de formação por causa de erros inerentes às aproximações usadas no desenvolvimento do formalismo. Para corrigir esses erros, nos métodos semi-empiricos o calor de formação é obtido a partir dos calores de formação atômicos (obtidos experimentalmente), sendo a energia de dissociação, $D$, descrita em sua versão empírica pela equação (4.54), e calculada segundo o método semiempírico (hamiltoniano) especifico.

$$
D=\sum_{A} E_{A}-E
$$

É dessa maneira que os métodos semi-empíricos são parametrizados para reproduzir calores de formação experimental em fase gasosa a temperatura de $298 \mathrm{~K}$ a partir de seus elementos constituintes no estado padrão.

\subsection{Método Semi-Empírico MNDO-PM3}

Os métodos semi-empíricos têm a mesma estrutura, são métodos $S C F-L C A O$ que levam em conta a repulsão eletrostática e a estabilização de troca (exchange), onde todas as integrais são calculadas de forma aproximada. Também fazem uso de funções de base mínima, um orbital $s$ e três orbitais $p\left(p_{x}, p_{y}\right.$ e $\left.p_{z}\right)$ por átomo. Existe na literatura uma série 
de métodos semi-empíricos nos quais mantém-se o formalismo SCF-LCAO e implementase uma série de parâmetros que são obtidos experimentalmente. Apresentaremos aqui informações básicas sobre o método MNDO-PM3 (Modified Neglet of Differential Overlap - Parametric Method Number 3) ${ }^{11}$ decorrente da aproximação ZDO. O calor de formação calculado pelo hamiltoniano MNDO-PM3 é dado pela equação (4.55),

$$
\Delta H_{f}=E_{e l e}+E_{n u c}-\sum_{A} E_{e l e}^{A}+\sum_{A} \Delta H_{f}^{A}
$$

Onde o terceiro termo e quarto termo da equação, descrevem o calor de formação na fase gasosas do átomo $A$ a partir de seus íons e o calor de formação experimental para o átomo $A$ repectivamente.

$$
E_{e l e}^{A}=f\left(P_{i i}, U_{i i},<i i|j j>,<i j| i j>\right)
$$

onde $P_{i i}$ representa a população do orbital atômico $i$ no estado fundamental. $U_{i i}$ são as energias de 1-elétron do orbital atômico do íon, resultante da remoção de todos seus elétrons de valência. $U_{i i}$ é representado pelos parâmetros $U_{s:}$ e $U_{p p .}$. $\left\langle\left. i i\right|_{j j}\right.$ > são as integrais de 2-elétrons em um centro. Em geral, existem cinco integrais deste tipo para cada átomo, representadas pelos seguintes parâmetros.

$$
\begin{aligned}
& G_{s s}=<s s \mid s s> \\
& G_{p p}=<p p \mid p p> \\
& H_{s p}=<s p \mid s p> \\
& G_{p p}=<p p \mid p p> \\
& G_{p 2}=<p p \mid p^{\prime} p^{\prime}>
\end{aligned}
$$

onde $p$ e $p$ ' descrevem os orbitais do tipo $\left(p_{x}, p_{y}, e p_{z}\right)$ no mesmo centro, porém com diferente orientação angular. $E_{n u c}$ é a energia de repulsão nuclear de caroço, dada por:

$$
E_{n u c}=\sum_{i<j} E_{N}(i, j)
$$

onde

$$
\begin{aligned}
& E_{N}(i, j)=Z_{A} Z_{B}<A A B B>\left(1+e^{\left(-\alpha_{A} R_{i j}\right)}+e^{\left(-\alpha_{B} R_{i j}\right)}\right)+ \\
& +\left(\frac{Z_{i} Z_{j}}{R_{i j}}\right)\left(\sum_{k} a_{K B} e^{-b K_{A}\left(R_{i j}-C_{K A}\right)}+\sum_{k} a_{k B} e^{-b k_{B}\left(R_{i j} C_{K B}\right)^{2}}\right)
\end{aligned}
$$


Os últimos dois termos representam funções exponenciais (duas por átomo). $\angle A A \mid B B>$ é a integral de 2-elétrons do tipo $\langle s s \mid s s\rangle, Z_{i}$ é o número de elétrons de valência do átomo $A$ e $R_{A B}$ é a distância interatômica. A energia eletrônica total é dada pela equação (4.31).

As quantidades $\alpha_{A}, a_{i k}, b_{i k}$ e $c_{i k}$ são parâmetros ajustados para reproduzir o calor de formação. Há variações na expresão (4.59) quando interações O-H e N-H estão envolvidas

12. Neste contexto a Matrix diagonal de Fock é defina pela seguinte expressão,

$$
\left.F_{\mu \mu}=U_{\mu \mu}+\sum_{B} V_{\mu \mu} B+\sum_{v} P_{v v} \cdot[\langle\mu \mu \mid v v\rangle-\langle\mu \nu \mid \mu \nu\rangle\rangle\right]+\sum_{B} \sum_{\lambda}^{B} \sum_{\sigma}^{B} P_{\lambda \sigma}\langle\mu \mu \mid \lambda \sigma\rangle
$$

onde $V_{\mu \mu B}$ é o potencial que o orbital atômico $\varphi_{\mu}$ experimenta devido a todos os núcleos do sistema. A matriz que descreve os elementos conectando orbitais em átomos diferentes é definida como,

$$
F_{\mu v}=\frac{S_{\mu v}\left(\beta_{\mu}+\beta_{v}\right)}{2}-\sum_{\lambda \sigma} P_{\lambda \sigma}<\mu \lambda|v \sigma\rangle
$$

onde $\beta_{\mu}$ e $\beta_{v}$ são parâmetros atômicos do tipo $\beta_{s}$ e $\beta_{p}$. As integrais do tipo $\langle\mu \lambda| v \sigma>$ são funções de Slater complicadas, as quais são parametrizadas. A mais simples delas é a que envolve somente orbitais $s$; todas as outras integrais envolvendo as funções $p$ são descritas com detalhes na referência ${ }^{\mathbf{1 3}}$.

$$
\left\langle s s \mid s^{\prime} s^{\prime}\right\rangle=\frac{27.21}{\sqrt{\frac{1}{R}+\frac{1}{4}\left(\frac{27.21}{G_{s s A}}+\frac{27.21}{G_{s s B}}\right)^{2}}}
$$

Para o hamiltoniano $M N D O-P M 3$ existem 18 parâmetros para serem otimizados para cada elemento, com exceção do átomo de hidrogêneo, o qual necessita de apenas 11 parâmetros. Os valores desses 18 parâmetros para 12 átomos são apresentados na Tabela 4.1. Esses parâmetros foram otimizados para reproduzir calores de formação experimentais de sistemas químicos de interesse. Embora não haja um formalismo não ambíguo para determinar se esses parâmetros correspondem ou não a um mínimo global, análises da aplicabilidade desses parâmetros para uma faixa grande de compostos sugerem que não existem outros mínimos de energia que sejam significantes. No próximo capítulo apresentaremos a metodologia adotada para calcular os parâmetros teóricos de energia livre, os quais serão usados para analisar a incorporação de solutos não iônicos em micelas. 


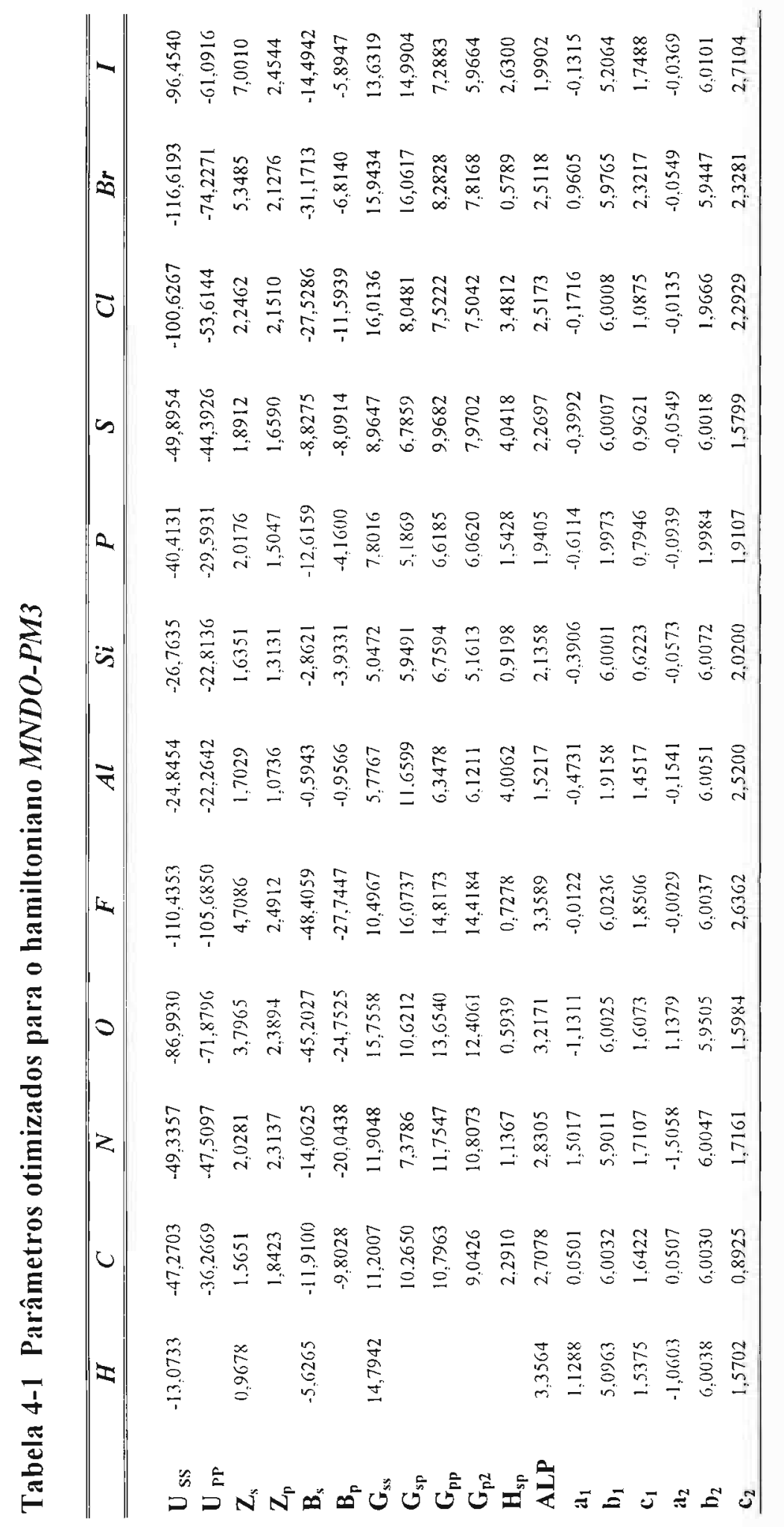




\section{Referências}

1. Helleraas, E.A.; 1929, J. Phyz.;54,347.

2. Hartree, D.R.; 1929, Proc. Cambridge Phil. Soc. 89.

3. Fock, V.; 1930, J. Phyz.; 61,1261.

4. Slater, J.C.; 1930, Phy. Rev.,35,210.

5. Roothaan, C.C.J.; 1951, Rev. Mod. Phy,; 26,69.

6. Bohr, N.; 1923, Proc. Phys. Soc.(London); 35,269.

7. Slater, J.C.;1963, Quantum Theory of Atomic Structure; vols. Ie 2;McGraw-Hill, New York.

8. Huzinaga, S.; 1985, Comp. Phys. Rep.; 2,80.

9. Parises, R.; Parr, R.G.; 1953, J.Chem.Phys.;21,466.

10. Pople, J.A.; Santry, D.P; Segal, G.A.;1963, J. Chem. Phys.;43,447.

11. Stewart, J.J.P.; 1989 J. Comp. Chem.;10,(2),221.

12. Dewar, M.J.S.; Thiel, W.,1977, J. Am. Chem. Soc.; $99,4899$.

13. Stewart, J.J.P.; 1993 MOPAC-93 Manual,Fujitsu Limited, Tokyo, Japan. 


\section{Capítulo 5}

Metodologia: Gálculo dos

Parâmetros Teóricos

Neste capítulo apresentamos a metodologia empregada para calcular os valores dos parâmetros teóricos (usando o hamiltoniano semi-empírico MNDO-PM3) para 693 solutos não iônicos distribuídos em 19 grupos funcionais. 


\subsection{Metodologia}

A metodologia adotada neste estudo usa a filosofia e a estrutura do modelo de Famini, porém fará uso de sete parâmetros teóricos para representar às interações de solutosolvente. Escolhemos os parâmetros de maneira a ter uma correspondência com os parâmetros experimentais de relação linear de energia livre de solvatação. Os sete parâmetros escolhidos são descritos a seguir.

\section{$5.2(V)$ - Volume do Soluto}

O parâmetro $V$ pode ser representado pelo volume de van der Waals $\left(V_{m v}\right)$, calculado diretamente da estrutura molecular através do método de Hopfinger ${ }^{1}$ (este método está implementado no programa $A M S O L-5.4$ ), ou poderá ser calculado pelo método de McGowan $^{2-4}\left(V_{x}\right)$, o qual considera o volume como sendo uma quantidade aditiva obtida a partir da estrutura molecular para qualquer tipo de soluto sólido, líquido ou gás. No caso do método de McGowan, o volume é calculado com o auxílio de um algorítmo para o cálculo do número de ligações químicas presentes na molécula, dado pela equação (5.1),

$$
N_{\text {lig }}=N_{\text {atom }}-(1+R)
$$

onde $N_{\text {lig }}$ é o número de ligações químicas, $N_{\text {atom }}$ é o número de átomos na molécula, e $R$ o número de anéis. Logo $V_{x}$ pode ser calculado pela equação (5.2)

$$
V_{x}=\sum_{i} N_{\text {atom }}^{i} \times V_{\text {atom }}^{i}-N_{\text {lig }} \times 6,56
$$

onde $N_{\text {atom }}^{i}$ é o número de cada tipo ( $i$ ) de átomo que compõe a molécula e $V_{\text {atom }}$ o volume atômico característico. Na Tabela 5.1 mostramos os valores do volume atômico, usados no cálculo do volume molecular. Note que na equação (5.2) é subtraído de cada ligação 6,56 $\mathrm{cm}^{3} \mathrm{~mol}^{-1}$. Esse valor corresponde ao volume da sobreposição das esferas que contém os átomos que participam da ligação química.

\section{Tabela 5.1 Volume atômico característico $\left(\mathrm{cm}^{3} \mathrm{~mol}^{-1}\right)$}

\begin{tabular}{lllllllllr}
\hline $\mathbf{C}$ & 16,39 & $\mathbf{N}$ & 14,39 & $\mathbf{O}$ & 12,43 & $\mathbf{F}$ & 10,48 & $\mathbf{H}$ & 8,71 \\
$\mathbf{S i}$ & 26,83 & $\mathbf{P}$ & 24,87 & S & 22,91 & $\mathbf{C l}$ & 20,95 & $\mathbf{B}$ & 18,32 \\
$\mathbf{G e}$ & 31,02 & As & 29,42 & Se & 27,81 & $\mathbf{B r}$ & 26,21 & & \\
Sn & 39,35 & Sb & 37,74 & Te & 36,41 & $\mathbf{I}$ & 34,53 & & \\
\hline
\end{tabular}

O algorítmo para o cálculo do número de ligações químicas tem a mesma limitação em distinguir ligações simples, duplas e triplas como no cálculo do volume de van der Walls, ou seja, os dois métodos estimam o mesmo valor para o volume de isômeros estruturais. 


\subsection{Interação por Pontes de Hidrogênio}

No modelo de Famini a interação por pontes de hidrogênio é separada em componentes do doador (caráter ácido) e do aceptor (caráter básico). Os parâmetros teóricos usados para descrever o caráter doador e aceptor são separados em duas componentes, uma covalente e outra eletrostática. Ao contrario de Famini, não usaremos a

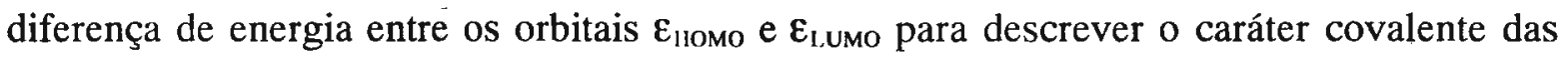
ligações mas sim $o$ valor da energia desses orbitais.

\subsection{1 ( $\left.q_{+}, \varepsilon_{L U M O}\right)$ Caráter ácido da interação}

A contribuição covalente para o caráter ácido da ponte de hidrogênio é descrita pelo parâmetro $\varepsilon_{\text {l.umo }}$ calculado pela equação (4.51), e a contribuição eletrostática é descrita pela magnitude da maior carga formal positiva $\left(q_{+}\right)$sobre o átomo de hidrogênio pertencente à molécula de soluto. Essa região da molécula descreve um centro atrativo de cargas negativas para propiciar a formação de pontes de hidrogênio.

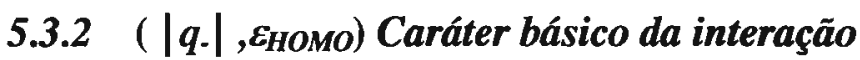

A contribuição covalente para o caráter básico da ponte de hidrogênio é descrita pelo

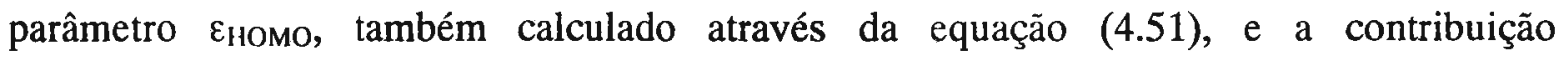
eletrostática é descrita pela magnitude do módulo da maior carga formal negativa $\mid q$ - $\mid$ sobre o heteroátomo da molécula do soluto. Esse orbital contém os elétrons que interagem com os prótons da vizinhança.

\subsubsection{Cálculo da carga formal}

Neste estudo faremos uso de uma ferramenta semi-empírica para o cálculo de carga formal chamada de Charge Model-1 - CM1 ${ }^{5}$ (esta ferramenta está implementada no programa $A M S O L-5.4$ ). O modelo $C M I$ transforma o conjunto de cargas obtido através da análise populacional de Mulliken $^{\mathbf{6}-8}$, (equação 4.34) em um novo conjunto de cargas parametrizado para reproduzir momentos de dipolo experimentais. Deste modo:

$$
q_{k}^{(M}=f\left(q_{k}^{(o)}, A, B, \ldots\right)
$$

onde $f$ é a funcional semi-empírica, $q_{k}$ é o conjunto inicial de cargas obtido pela análise populacional de Mulliken e $A$ e $B$ são parâmetros ajustados para reproduzir momentos de dipolo experimentais sendo,

$$
q_{k}^{(0)}=Z_{k}-\sum_{\mu \in k} P_{\mu \mu}
$$

onde $Z_{k}$ é a carga nuclear menos o número de elétrons de caroço, $\mu$ são os índices das funções de base e $\boldsymbol{P}_{\mu \mu}$ são os elementos diagonais da matriz de densidade. A transformação 
de um conjunto de cargas iniciais é feita em dois passos. Primeiro, as cargas parciais iniciais são ajustadas usando a seguinte relação,

$$
q_{k}^{(1)}=q_{k}^{(0)}+B_{k} \Delta q_{k}
$$

onde

$$
\Delta q_{k}=c_{k} q_{k}^{(o)}+d_{K}
$$

e onde $c_{k}$ é o fator de escala, $d_{k}$ a constante e $B_{k}$ a soma dos elementos da matriz de ordem de ligação do átomo $k$ em relação aos outros átomos. Os parâmetros $c_{k}$ e $d_{k}$ assumem a seguinte forma geral,

$$
c_{k}=\hat{c}_{k}+\sum_{k^{\prime} \neq k} f^{(c)}\left(B_{k k^{\prime}}\right) c_{k k^{\prime}}
$$

e

$$
d_{k}=\hat{d}_{k}+\sum_{k^{\prime} \neq k} f^{(d)}\left(B_{k k^{\prime}}\right) d_{k k^{\prime}}
$$

Nas equações (5.7) e (5.8), $c_{k}$ e $d_{k}$ são constantes que dependem do número atômico do átomo $k, B_{k k^{\prime}}$, é a ordem de ligação entre os átomos $k$ e $k^{\prime}, f^{(c)}\left(B_{k k^{\prime}}\right)$ e $f^{(d)}\left(B_{k k}\right)$, são funções de $\left(B_{k k^{\prime}}\right),\left(C_{k k^{\prime}}\right)$, e $\left(d_{k k^{\prime}}\right)$ que dependem do número atômico dos átomos $k$ e $k^{\prime}$. No segundo passo as cargas parciais são ajustadas para reproduzir a carga total da molécula, a carga formal final sendo dada por:

$$
q_{k}=q_{k}^{(o)}+B_{k} \Delta q_{k}-\sum_{k^{\prime} \neq k} B_{k k^{\prime}} \Delta q_{k^{\prime}}
$$

onde $\left(B_{k k^{\prime}}\right)$ é a ordem de ligação entre os átomos $k$ e $k^{\prime}$. A soma das ordens de ligação a partir do átomo $k$ até todos os outros átomos que compõe a molécula é dado por,

$$
B_{k}=\sum_{k^{\prime} \neq k} B_{k k^{\prime}}
$$

Nesta metodologia qualquer definição de ordem de ligação pode ser usada nas equações acima. Porém, neste estudo usamos o índice de ligação covalente como definido por Armstrong et al. ${ }^{9}$, ou seja,

$$
B_{k k^{\prime}}=\sum_{\mu \in k} \sum_{\mu \in k^{\prime}} P_{\mu \nu}^{2}
$$

Cargas formais calculadas usando o método $C M I$ são mais precisas que aquelas determinadas pela análise populacional de Mullliken, e possuem precisão similar aquelas calculadas por métodos ab-initio Chelp $G$ usando funções de base $6-31 G^{* 10}$. Como a carga atômica não é uma quantidade rigorosamente definda em mecânica quântica, existem outras formas de obtê-la, como por exemplo através do ajuste do potencial eletrostático (ESP - 
EletroStatic Potential), através do cálculo direto da função de onda" ou calculando a densidade de carga a partir da função de onda e depois integrando a equação de Poisson ${ }^{12}$; porém, neste estudo abordaremos somente as cargas formais calculadas usando o método $C M 1$.

\section{$5.4(\mu)$ Momento de Dipolo}

O momento de dipolo $(\mu)$ pode ser calculado usando-se tanto as cargas formais obtidas diretamente a partir da análise populacional de Mulliken quanto usando as cargas formais obtidas pelo método $C M 1$, sendo calculado pela seguinte equação:

$$
\mu=\left[\left(\sum \mathrm{q}_{\mathrm{k}} \mathrm{r}_{\mathrm{k}}\right)^{2}\right]^{1 / 2}
$$

onde $r_{k}$ e $q_{k}$ são as coordenadas cartesianas e as cargas formais sobre cada átomo respectivamente. Para comparação mostramos na Tabela 5.2 os valores experimentais e calculados do momento de dipolo. Os valores foram calculados através do hamiltoniano $M N D O-P M 3$ usando os métodos CM1 e diretamente a apartir da análise populacional de Mulliken.

Tabela 5.2 Momento de dipolo de solutos contendo átomos de $C, N, H, O, I, B r, F e C l$.

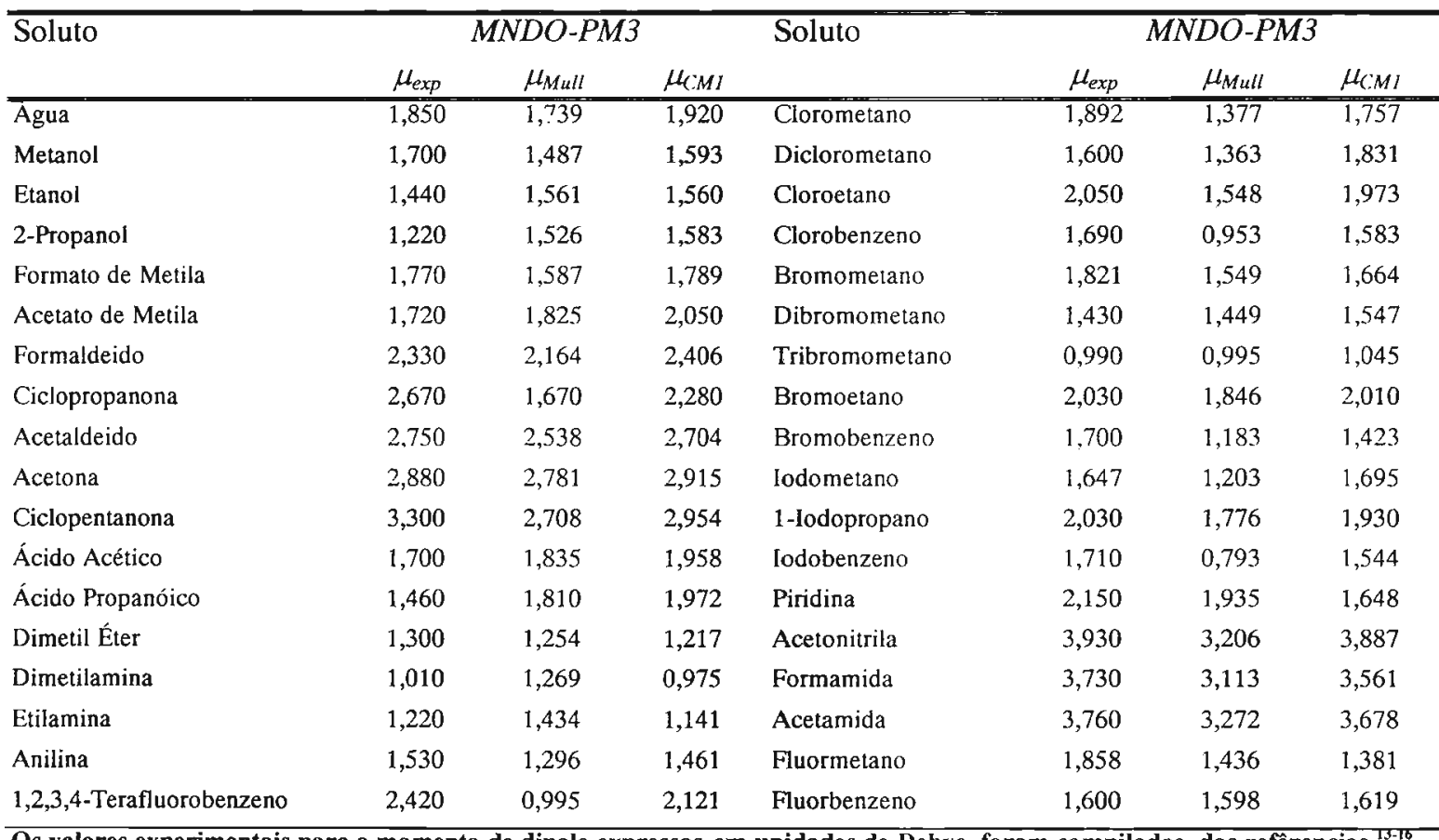

Os valores experimentais para o momento de dipolo expressos em unidades de Debye, foram compilados das refêrencias ${ }^{13-16}$.

Neste estudo somente usaremos o momento de dipolo obtido a partir de cargas formais calculadas pelo método $C M 1$ por que a magnitude da cargas cargas formais calculadas desta forma é mais próxima dos valores determinados experimentalmente. Quanto maior o valor do momento de dipolo indica que moléculas do soluto tem uma maior tendência a criar um ambiente polarizavél. 


\section{$5.5\left(\pi_{F}\right)$ Dipolaridade/Polarizabilidade}

O parâmetro de dipolaridade/polarizabilidade pode ser descrito pelo índice $\left(\pi_{F}\right)$, o qual é obtido dividindo o valor calculado da polarizabilidade (usando o método de Kurtz, ${ }^{17}$ como implementado no programa $M O P A C-93)$, incluindo ou não correções atômicas ${ }^{18}$, dividido pelo volume (calculado pelo método de McGowan ou pelo método de Hopfinger) do soluto. Desta forma, obtem-se uma quantidade adimencional e independente do tamanho da molécula que indica a facilidade com que uma densidade de carga é polarizada e a tendência da fase interagir com solutos polar e/ou polarizavel.

$$
\pi_{F}=\frac{a}{V}
$$

\subsubsection{Cálculo semi-empírico de polarizabilidade $(\alpha)$}

Quando um campo elétrico $F(\lambda)$ é aplicado a um átomo ou molécula, a distribuição dos elétrons é modificada e a geometria molecular distorcida. Átomos e moléculas respondem ao campo elétrico, adquirindo um momento de dipolo elétrico induzido (somado ao momento de dipolo permanente no caso de moléculas polares). A polarizabilidade $(\alpha)$ é uma constante de proporcionalidade entre o campo elétrico aplicado e o momento de dipolo induzido $\left(\mu^{\prime}\right)$, ou seja,

$$
\mu^{\prime}=\alpha F(\lambda)
$$

A polarizabilidade total de uma molécula é composta de várias contribuições. A polarizabilidade atômica é a contribuição devido à distorção geométrica, sendo significativamente menor que a polarizabilidade eletrônica proveniente do deslocamento da distribuição eletrônica. A polarizabilidade de orientação é a contribuição devido a modificação média da orientação adotada por molécula polares sob a ação do campo elétrico, logo a polarizabilidade depende da freqüência do campo aplicado. Para baixas frequiências $\left(10^{12} \mathrm{~Hz}\right.$, ou da ordem de grandeza da taxa de rotação das moléculas) moléculas polares orientam-se na direção do campo aplicado e todas as contribuições para a polarizabilidade total estão presentes. Para freqüências acima de $10^{12} \mathrm{~Hz}$, as moléculas não conseguem se orientar a tempo e a polarizabilidade orientacional deixa de contribuir. Para frequências ainda maiores (acima de $10^{14} \mathrm{~Hz}$, ou da ordem de grandeza da taxa de vibração das moléculas) os núcleos atômicos também não respondem à oscilação do campo e a contribuição atômica deixa de contribuir. A freqüências maiores (na região do visível) somente as contribuições eletrônicas contribuem para a polarizabilidade total. Logo cálculos de polarizabilidade dependente da freqüência são importantes para distinguir as várias contribuições.

A variação $\Delta \boldsymbol{E}$ na energia $(\boldsymbol{E})$ da molécula quando calculada sob a influência do campo elétrico $F(\lambda)$ pode ser expresso através de uma série de Taylor,

$$
\frac{\partial E}{\partial F}=\left(\frac{\partial E}{\partial F}\right) F+\left(\frac{\partial^{2} E}{\partial F^{2}}\right) F^{2}+\left(\frac{\partial^{3} E}{\partial F^{3}}\right) F^{3}+\ldots \ldots
$$


Quando o momento de dipolo permanente $(\mu)$ de uma molécula é orientado na direção do campo aplicado, sua energia é modificada pela quantidade $F \mu$. Comparando este termo com as equações (5.15) temos:

$$
\mu=\frac{\partial E}{\partial F}
$$

A polarizabilidade de uma molécula mede o momento de dipolo induzido quando um campo elétrico $F(\lambda)$ é aplicado, ou seja:

$$
\alpha=\frac{\partial \mu}{\partial F}
$$

Das equações (5.15) e (5.16) temos:

$$
\alpha=\frac{\partial^{2} E}{\partial F^{2}}
$$

Se o campo elétrico aplicado for de intensidade alta o momento de dipolo induzido dependerá de variações não lineares chamadas de hiperpolarizabilidade. Neste estudo estamos interessados somente no cálculo da polarizabilidade $(\alpha)$. A polarizabilidade tem unidade de volume e é frequêntemente chamada de polarizabilidade volumétrica.

\subsubsection{Procedimento MNDO-PM3 para cálculo da polarizabilidade}

Os elementos de matriz de Fock $\left(\boldsymbol{F}_{\boldsymbol{\mu}}\right)$ na presença de campo elétrico são descritos pela seguinte equação

$$
F_{\mu v}=F_{\mu v}^{(0)}-\sum_{i} F_{i} D_{\mu \nu}^{i}
$$

onde $F_{i}$ é a i-ésimo elemento do vetor campo elétrico e $D_{\mu \nu}$ são as correspondentes componentes da matriz de momento de dipolo entre os orbitais atômicos $\varphi_{\mu}$ e $\varphi_{\nu}$;

$$
D_{\mu v}^{i}=e\left\langle\mu\left|r_{i}\right| v\right\rangle
$$

No hamiltoniano $M N D O-P M 3, D_{\mu \nu}$ é negligenciada, a menos que os orbitais $\varphi_{\mu}$ e $\varphi_{\nu}$ estejam centradas no mesmo átomo. A componente para a polarizabilidade é descrita por,

$$
\alpha_{i j}=-\sum_{\mu} \sum_{v}\left(\frac{\partial P_{\mu \nu}}{\partial F_{j}}\right) D_{\mu \nu}^{i}
$$

onde $P_{\mu \nu}$ é a matriz de ordem de ligação. Os cálculos são feitos para a geometria de equilíbrio. Valores experimentais $\alpha_{\exp }(\lambda)$ são usualmente maiores que os valores calculados por métodos semi-empíricos, pois o procedimento $S C F-L C A O$, usando um conjunto de base mínima, subestima a polarizabilidade atômica. Como o valor da polarizabilidade atômica 
independe da molécula no qual o átomo aparece, é possível fazer uma correção tratando as diferenças $\left(\delta \alpha_{i}\right)$ entre o valor experimental e o calculado como parâmetro através de uma regressão linear, como indica a equação abaixo,

$$
\alpha_{\exp } \approx \alpha_{C a l}^{M N D O-P M 3}+\sum_{i} \delta \alpha_{\text {atom }}^{i}
$$

onde

$$
\alpha_{C a l}=\sum_{i} \alpha_{i}^{a l o m}+\alpha_{C a l}^{M N D O-P M 3}
$$

sendo que $\Sigma \alpha_{i}^{\text {atom }}$ descreve a contribuição atômica dos átomos (i) que compõe a molécula e $\alpha_{C a l}^{M N D O-P M 3}$ a contribuição devido a formação de ligações químicas calculada segundo o procedimento semi-empírico descrito acima. Para comparação, mostramos na Tabela 5.3 os valores de $\left(\delta \alpha_{i}\right)$ obtidos por regressão linear através da equação (5.22) e os valores experimentais da polarizabilidade atômica $\left(\boldsymbol{\alpha}_{i}\right)$. Este procedimento é uma maneira simples de estimar a polarizabilidade de moléculas neutras. Para calcular a correção da polarizabilidade atômica, desenvolvemos um algoritmo que, fornecendo a fórmula química da molécula, calcula a correção atômica da polarizabilidade e adiciona esse valor ao calculado pelo hamiltoniano escolhido.

Tabela 5.3 Correção atômica para a polarizabilidade

\begin{tabular}{lcc}
\hline Atomo & $\delta \alpha_{i}\left(\AA^{3}\right)$ & $\alpha_{i} \exp \left(\AA^{3}\right)$ \\
\hline Hidrogênio & 0,2287 & 0,1917 \\
Carbono & $\mathbf{0 , 2 6 4 7}$ & 1,5549 \\
Nitrogênio & $\mathbf{0 , 3 5 8 4}$ & 1,2856 \\
Oxigênio & $\mathbf{0 , 2 3 2 4}$ & 0,6034 \\
Flúor & 0,1982 & 0,3318 \\
Cloro & $\mathbf{1 , 3 2 3 6}$ & 2,2550 \\
Bromo & 2,2583 & 3,3862 \\
lodo & 4,0930 & 4,0930 \\
\hline
\end{tabular}

Os valores calculados através do hamiltoniano $M N D O-P M 3$ para a polarizabilidade molecular considerando campo estático $\left(\alpha_{0}\right)$ e dependente da frequência $\alpha(\lambda)$ e as respectivas correções atômicas são apresentados no Tabela 5.4. Os valores calculados estão de acordo com os valores obtidos esperimentalmente, usando a equação de ClausiusMossoti, a qual descreve uma relação entre o índice de refração de uma solução e sua polarizabilidade.

$$
\alpha(\lambda)=\frac{3}{4 \pi N} V_{x}\left(\frac{n^{2}-1}{n^{2}+2}\right) \times 10^{24} A^{3}
$$

onde $N$ é o número de Avogadro, $n$ o índice de refração do meio, e $\lambda$ o comprimento de onda ( $\lambda=5893, \AA$ linha $D$ do sódio $)$. 
Tabela 5.4 Valores da polarizabilidade molecular calculada a partir do hamiltoniano semie-mpírico $M N D O-P M 3$ incluindo correções atômica.

\begin{tabular}{|c|c|c|c|c|c|c|c|c|}
\hline No & Soluto & Fórmula & $\alpha_{D}\left(A^{3}\right)$ & $\alpha_{0}\left(\AA^{3}\right)$ & $\bar{\Sigma} \alpha_{i}\left(\AA^{3}\right)$ & $u_{\text {calc }(D)}\left(\AA^{3}\right)$ & $\alpha_{\text {calc (v) }}\left(\AA^{3}\right)$ & $\alpha_{\text {exp }}\left(\AA^{3}\right)$ \\
\hline \multicolumn{9}{|c|}{ Agua, Alcoois e Tióis } \\
\hline s352 & 2-octanol & $\mathrm{C}_{8} \mathrm{H}_{18} \mathrm{O}$ & 9,456 & 8,939 & 6,467 & 15,923 & 15,405 & \\
\hline s367 & 2-hexafluorpropanol & $\mathrm{C}_{3} \mathrm{H}_{2} \mathrm{OF}_{8}$ & 4,206 & 4,007 & 2,673 & 6,879 & 6,680 & \\
\hline s366 & 2,2,2-trifluorpetanol & $\mathrm{C}_{2} \mathrm{H}_{3} \mathrm{OF}_{3}$ & 2,975 & 2,829 & 2,043 & 5,018 & 4,872 & \\
\hline s365 & 2-propeno-1-ol & $\mathrm{C}_{3} \mathrm{H}_{6} \mathrm{O}$ & 4,656 & 4,208 & 2,399 & 7,055 & 6,606 & \\
\hline s364 & 1-adamantanol & $\mathrm{C}_{10} \mathrm{H}_{16} \mathrm{O}$ & 10,884 & 10,305 & 6,539 & 17,423 & 16,843 & \\
\hline s363- & Ciclooctanol & $\mathrm{C}_{8} \mathrm{H}_{16} \mathrm{O}$ & 9,197 & 8,698 & 6,009 & 15,206 & 14,708 & \\
\hline s362 & Cicloheptanol & $\mathrm{C}_{7} \mathrm{H}_{14} \mathrm{O}$ & 8,077 & 7,640 & 5,287 & 13,364 & 12,927 & \\
\hline$s 361$ & Ciclohexanol & $\mathrm{C}_{6} \mathrm{H}_{12} \mathrm{O}$ & 7,065 & 6,680 & 4,565 & 11,630 & 11,245 & \\
\hline s368 & 2-cloroetanol & $\mathrm{C}_{2} \mathrm{H}_{5} \mathrm{OCl}$ & 3,999 & 3,743 & 3,229 & 7,228 & 6,972 & \\
\hline $\mathrm{s} 360$ & Ciclopentanol & $\mathrm{C}_{5} \mathrm{H}_{10} \mathrm{O}$ & 5,822 & 5,513 & 3,843 & 9,665 & 9,356 & \\
\hline s359 & 1-tetradecanol & $\mathrm{C}_{14} \mathrm{H}_{30} \mathrm{O}$ & 16,150 & 15,251 & 10,799 & 26,949 & 26,050 & \\
\hline s358 & 1-tridecanol & $\mathrm{C}_{13} \mathrm{H}_{28} \mathrm{O}$ & 15,038 & 14,202 & 10,077 & 25,115 & 24,279 & \\
\hline s357 & 1-dodecanol & $\mathrm{C}_{12} \mathrm{H}_{26} \mathrm{O}$ & 13,926 & 13,153 & 9,355 & 23,281 & 22,508 & \\
\hline s356 & 1-undecanol & $\mathrm{C}_{11} \mathrm{H}_{24} \mathrm{O}$ & 12,816 & 12,105 & 8,633 & 21,449 & 20,738 & \\
\hline s355 & 1-decanol & $\mathrm{C}_{10} \mathrm{H}_{22} \mathrm{O}$ & 11,705 & 11,057 & 7,911 & 19,616 & 18,968 & \\
\hline s354 & 1-nonanol & $\mathrm{C}_{9} \mathrm{H}_{20} \mathrm{O}$ & 10,595 & 10,010 & 7,189 & 17,784 & 17,198 & \\
\hline s370 & 2-etoxietanol & $\mathrm{C}_{4} \mathrm{H}_{10} \mathrm{O}_{2}$ & 5,770 & 5,458 & 3,811 & 9,580 & 9,269 & \\
\hline s369 & 2-metoxietanol & $\mathrm{C}_{3} \mathrm{H}_{8} \mathrm{O}_{2}$ & 4,595 & 4,349 & 3,089 & 7,684 & 7,438 & \\
\hline s353 & 4-octanol & $\mathrm{C}_{8} \mathrm{H}_{18} \mathrm{O}$ & 9,471 & 8,952 & 6,467 & 15,938 & 15,418 & \\
\hline s335 & 2-metil-1-butanol & $\mathrm{C}_{5} \mathrm{H}_{12} \mathrm{O}$ & 6,122 & 5,791 & 4,300 & 10,422 & 10,091 & \\
\hline s334 & 3-pentanol & $\mathrm{C}_{5} \mathrm{H}_{12} \mathrm{O}$ & 6,132 & 5,801 & 4.300 & 10,432 & 10,101 & \\
\hline s333 & 2-pentanol & $\mathrm{C}_{5} \mathrm{H}_{12} \mathrm{O}$ & 6,124 & 5,794 & 4,300 & 10,425 & 10,095 & \\
\hline s332 & 1-pentanol & $\mathrm{C}_{4} \mathrm{H}_{12} \mathrm{O}$ & 6,163 & 5,825 & 4,300 & 10,463 & 10,125 & \\
\hline s331 & 2-metil-2-propanol & $\mathrm{C}_{4} \mathrm{H}_{10} \mathrm{O}$ & 4,931 & 4,678 & 3,578 & 8,509 & 8,256 & \\
\hline s330 & 2-butanol & $\mathrm{C}_{4} \mathrm{H}_{10} \mathrm{O}$ & 5,014 & 4,747 & 3,578 & 8,592 & 8,325 & \\
\hline s329 & 2-metil-1-propanol & $\mathrm{C}_{4} \mathrm{H}_{10} \mathrm{O}$ & 4,984 & 4,716 & 3,578 & 8,562 & 8,294 & \\
\hline s328 & 1-butanol & $\mathrm{C}_{4} \mathrm{H}_{10} \mathrm{O}$ & 5,058 & 4,782 & 3,578 & 8,637 & 8,360 & \\
\hline s327 & 2-propanol & $\mathrm{C}_{3} \mathrm{H}_{8} \mathrm{O}$ & 3,916 & 3,709 & 2,856 & 6,772 & 6,566 & \\
\hline s326 & 1-propanol & $\mathrm{C}_{3} \mathrm{H}_{8} \mathrm{O}$ & 3,953 & 3,737 & 2,856 & 6,809 & 6,593 & \\
\hline s325 & Etanol & $\mathrm{C}_{2} \mathrm{H}_{6} \mathrm{O}$ & 2,836 & 2,683 & 2.134 & 4.970 & 4,817 & \\
\hline s324 & Metanol & $\mathrm{CH}_{4} \mathrm{O}$ & 1,714 & 1,622 & 1,412 & 3,126 & 3,034 & \\
\hline $\mathrm{s} 323$ & Água & $\mathrm{H}_{2} \mathrm{O}$ & 0,520 & 0,501 & 0,690 & 1,210 & 1,190 & 1,450 \\
\hline s344 & 4-metil-2-pentanol & $\mathrm{C}_{4} \mathrm{H}_{14} \mathrm{O}$ & 7,212 & 6,825 & 5,022 & 12,235 & 11,847 & \\
\hline $\mathrm{s} 351$ & 1-octanol & $\mathrm{C}_{4} \mathrm{H}_{18} \mathrm{O}$ & 9,486 & 8,962 & 0,467 & 15,952 & 15,429 & \\
\hline s350 & 4-heptanol & $\mathrm{C}_{7} \mathrm{H}_{16} \mathrm{O}$ & 8,356 & 7,900 & 5,745 & 14,101 & 13,644 & \\
\hline s349 & 3-heptanol & $\mathrm{C}_{7} \mathrm{H}_{16} \mathrm{O}$ & 8,354 & 7,898 & 5,745 & 14,098 & 13,642 & \\
\hline s348 & 2-heptanol & $\mathrm{C}_{7} \mathrm{H}_{16} \mathrm{O}$ & 8,338 & 7,884 & 5,745 & 14,083 & 13,629 & \\
\hline s347 & 1-heptanol & $\mathrm{C}_{7} \mathrm{H}_{10} \mathrm{O}$ & 8,378 & 7,916 & 5,745 & 14,122 & 13,661 & \\
\hline s346 & 2,3-dimetil-2-butanol & $\mathrm{C}_{6} \mathrm{H}_{14} \mathrm{O}$ & 7,146 & 6,771 & 5,022 & 12,169 & 11,794 & \\
\hline s336 & 3-metil-1-butanol & $\mathrm{C}_{5} \mathrm{H}_{12} \mathrm{O}$ & 6,130 & 5,799 & 4.300 & 10,430 & 10,099 & \\
\hline s345 & 2-metil-3-pentanol & $\mathrm{C}_{6} \mathrm{H}_{14} \mathrm{O}$ & 7,218 & 6,830 & 5,022 & 12,240 & 11,852 & \\
\hline s343 & 2-metil-2-pentanol & $\mathrm{C}_{6} \mathrm{H}_{14} \mathrm{O}$ & 7,172 & 6,793 & 5,022 & 12,194 & 11,815 & \\
\hline s342 & 3-hexanol & $\mathrm{C}_{6} \mathrm{H}_{14} \mathrm{O}$ & 7,243 & 6,850 & 5,022 & 12,265 & 11,872 & \\
\hline s341 & 2-hexanoi & $\mathrm{C}_{6} \mathrm{H}_{14} \mathrm{O}$ & 7,227 & 6,836 & 5,022 & 12,250 & 11,858 & \\
\hline s340 & 1-hexanol & $\mathrm{C}_{6} \mathrm{H}_{14} \mathrm{O}$ & 7,271 & 6,871 & 5,022 & 12,294 & 11,894 & \\
\hline s339 & 2,2-dimetil-1-propanol & $\mathrm{C}_{5} \mathrm{H}_{12} \mathrm{O}$ & 6,024 & 5,706 & 4,300 & 10,325 & 10,006 & \\
\hline
\end{tabular}




\section{Tabela 5.4 (continuação)}

\begin{tabular}{|c|c|c|c|c|c|c|c|c|}
\hline No & Soluto & Fórmula & $\alpha_{D}\left(A^{3}\right)$ & $\alpha_{0}\left(\AA^{3}\right)$ & $\sum \alpha_{i}\left(\AA^{3}\right)$ & $\alpha_{\text {calc (D) }}\left(\AA^{3}\right)$ & $\alpha_{\text {calc (o) }}\left(A^{3}\right)$ & $\alpha_{\text {exp }}\left(A^{3}\right)$ \\
\hline s338 & 3-metil-2-butanol & $\mathrm{C}_{5} \mathrm{H}_{12} \mathrm{O}$ & 6,067 & 5,745 & 4,300 & 10,367 & 10,046 & \\
\hline s337 & 2-metil-2-butanol & $\mathrm{C}_{5} \mathrm{H}_{12} \mathrm{O}$ & 6,059 & 5,742 & 4.300 & 10,360 & 10,042 & \\
\hline s656 & 1-feniletanol & $\mathrm{C}_{8} \mathrm{H}_{10} \mathrm{O}$ & 10,423 & 9,552 & 4,037 & 15,060 & 14,189 & \\
\hline s655 & 4-metilbenzil álcool & $\mathrm{C}_{8} \mathrm{H}_{10} \mathrm{O}$ & 10,840 & 9,846 & 4.637 & 15,477 & 14,483 & \\
\hline s654 & 3-metilbenzil álcool & $\mathrm{C}_{8} \mathrm{H}_{10} \mathrm{O}$ & 10,758 & 9,796 & 4.637 & 15,395 & 14,433 & \\
\hline s653 & Álcool benzílico & $\mathrm{C}_{7} \mathrm{H}_{8} \mathrm{O}$ & 9,336 & 8,525 & 3.915 & 13,251 & 12,440 & \\
\hline s657 & 2-feniletanol & $\mathrm{C}_{8} \mathrm{H}_{10} \mathrm{O}$ & 10,475 & 9,587 & +.637 & 15,112 & 14,224 & \\
\hline s658 & 3-fenilpropanol & $\mathrm{C}_{9} \mathrm{H}_{12} \mathrm{O}$ & 11,696 & 10,724 & 5.359 & 17,055 & 16,083 & \\
\hline \multicolumn{9}{|c|}{ Aldeídos } \\
\hline s502 & 2-metilbenzaldeído & $\mathrm{C}_{8} \mathrm{H}_{8} \mathrm{O}$ & 11,002 & 9,908 & 4,180 & 15,181 & 14,088 & \\
\hline s505 & 4-clorobenzaldeído & $\mathrm{C}_{7} \mathrm{H}_{5} \mathrm{OCl}$ & 11,645 & 10,295 & 4,552 & 16,197 & 14,847 & \\
\hline s504 & 4-metilbenzaldeído & $\mathrm{C}_{8} \mathrm{H}_{8} \mathrm{O}$ & 11,190 & 10,028 & 4,180 & 15,370 & 14,207 & \\
\hline s503 & 3-metilbenzaldeído & $\mathrm{C}_{8} \mathrm{H}_{8} \mathrm{O}$ & 10,994 & 9,903 & 4,180 & 15,173 & 14,083 & \\
\hline s501 & Benzaldeído & $\mathrm{C}_{7} \mathrm{H}_{6} \mathrm{O}$ & 9,584 & 8,645 & 3,458 & 13,041 & 12,102 & 12,8 \\
\hline$s 676$ & Paraldeído & $\mathrm{C}_{6} \mathrm{H}_{12} \mathrm{O}_{3}$ & 8,451 & 8,013 & 5,030 & 13,481 & 13,043 & \\
\hline s172 & Propanal & $\mathrm{C}_{3} \mathrm{H}_{6} \mathrm{O}$ & 3,883 & 3,652 & 2,399 & 6,282 & 6,051 & \\
\hline s171 & Acetaldeído & $\mathrm{C}_{2} \mathrm{H}_{4} \mathrm{O}$ & 2,761 & 2,596 & 1,677 & 4,438 & 4,273 & 4,57 \\
\hline s174 & Isobutanal & $\mathrm{C}_{4} \mathrm{H}_{8} \mathrm{O}$ & 4,948 & 4,661 & 3,121 & 8,069 & 7,782 & \\
\hline s173 & Butanal & $\mathrm{C}_{4} \mathrm{H}_{8} \mathrm{O}$ & 5,001 & 4,707 & 3,121 & 8,122 & 7,828 & \\
\hline s178 & Octanal & $\mathrm{C}_{8} \mathrm{H}_{16} \mathrm{O}$ & 9,448 & 8,904 & 6,009 & 15,457 & 14,913 & \\
\hline s180 & trans-2-butanal & $\mathrm{C}_{4} \mathrm{H}_{8} \mathrm{O}$ & 5,672 & 5,104 & 3,121 & 8,793 & 8,224 & \\
\hline s179 & Nonanal & $\mathrm{C}_{9} \mathrm{H}_{18} \mathrm{O}$ & 10,559 & 9,952 & 6,731 & 17,291 & 16,684 & \\
\hline s175 & Pentanal & $\mathrm{C}_{S} \mathrm{H}_{10} \mathrm{O}$ & 6,105 & 5,750 & 3,843 & 9,948 & 9,593 & \\
\hline s176 & Hexanal & $\mathrm{C}_{6} \mathrm{H}_{12} \mathrm{O}$ & 7,227 & 6,807 & 4.565 & 11,792 & 11,372 & \\
\hline s177 & Heptanal & $\mathrm{C}_{7} \mathrm{H}_{14} \mathrm{O}$ & 8,337 & 7,855 & 5,287 & 13,624 & 13,142 & \\
\hline s170 & Formaldeído & $\mathrm{CH}_{2} \mathrm{O}$ & 1,569 & 1,469 & 0,955 & 2,524 & 2,424 & \\
\hline \multicolumn{9}{|c|}{ Hidrocarbonetos Alifáticos } \\
\hline s048 & Metilciclopentano & $\mathrm{C}_{6} \mathrm{H}_{\mathrm{I} 2}$ & 6,386 & 6,040 & 4,333 & 10,718 & 10,372 & \\
\hline s049 & Ciclohexano & $\mathrm{C}_{6} \mathrm{H}_{12}$ & 6,569 & 6,201 & 4.333 & 10,902 & 10,534 & \\
\hline s024 & 2-metilpentano & $\mathrm{C}_{6} \mathrm{H}_{\mathrm{I}}$ & 6,711 & 6,342 & 4,790 & 11,501 & 11,132 & \\
\hline $\mathrm{s} 050$ & Metilciclohexano & $\mathrm{C}_{7} \mathrm{H}_{14}$ & 7,628 & 7,205 & 5,055 & 12,682 & 12,260 & \\
\hline s023 & $n$-hexano & $\mathrm{C}_{6} \mathrm{H}_{14}$ & 6,745 & 6,369 & 4,790 & 11,535 & 11,159 & 11,76 \\
\hline s046 & Ciclopropano & $\mathrm{C}_{3} \mathrm{H}_{6}$ & 3,275 & 3,090 & 2,166 & 5,441 & 5,256 & \\
\hline $\mathrm{s} 025$ & 3-metilpentano & $\mathrm{C}_{6} \mathrm{H}_{14}$ & 0,698 & 6,332 & 4.790 & 11,488 & 11,122 & \\
\hline s045 & $n$-tetradecano & $\mathrm{C}_{14} \mathrm{H}_{30}$ & 15,615 & 14,741 & 10,567 & 26,182 & 25,308 & \\
\hline s047 & Ciclopentano & $\mathrm{C}_{5} \mathrm{H}_{10}$ & 5,300 & 5,012 & 3.611 & 8,911 & 8,623 & \\
\hline s055 & Eteno & $\mathrm{C}_{2} \mathrm{H}_{4}$ & 2,397 & 2,223 & 1,444 & 3,842 & 3,667 & 4,26 \\
\hline s021 & 2-metilbutano & $\mathrm{C}_{5} \mathrm{H}_{12}$ & 5,598 & 5,292 & 4,068 & 9,665 & 9,359 & \\
\hline s051 & cis-1,2-dimetilciclohexano & $\mathrm{C}_{8} \mathrm{H}_{16}$ & 8,684 & 8,209 & 5,777 & 14,461 & 13,986 & \\
\hline s057 & 1 -buteno & $\mathrm{C}_{4} \mathrm{H}_{8}$ & 4,842 & 4,491 & 2,888 & 7,730 & 7,379 & \\
\hline s020 & $n$-pentano & $\mathrm{C}_{5} \mathrm{H}_{12}$ & 5,641 & 5,327 & 4,068 & 9,709 & 9,394 & 10,2 \\
\hline s056 & Propeno & $\mathrm{C}_{3} \mathrm{H}_{6}$ & 3,736 & 3,444 & 2,166 & 5,902 & 5,610 & \\
\hline s022 & 2,2-dimetilpropano & $\mathrm{C}_{5} \mathrm{H}_{12}$ & 5,484 & 5,193 & 4,068 & 9,552 & 9,261 & \\
\hline s052 & trans-1,4-dimetilciclohexano & $\mathrm{C}_{8} \mathrm{H}_{16}$ & 8,690 & 8,213 & 5,777 & 14,466 & 13,990 & \\
\hline s054 & Adamanteno & $\mathrm{C}_{10} \mathrm{H}_{16}$ & 10,405 & 9,839 & 6,306 & 16,711 & 16,146 & \\
\hline s053 & Ciclooctano & $\mathrm{C}_{8} \mathrm{H}_{16}$ & 8,687 & 8,207 & 5,777 & 14,464 & 13,984 & \\
\hline s030 & 3-metilhexano & $\mathrm{C}_{7} \mathrm{H}_{16}$ & 7,818 & 7,386 & 5,512 & 13,330 & 12,898 & \\
\hline s036 & 3-metilheptano & $\mathrm{C}_{8} \mathrm{H}_{18}$ & 8,925 & 8,432 & 6,234 & 15,159 & 14,666 & \\
\hline s029 & 2-metilhexano & $\mathrm{C}_{7} \mathrm{H}_{16}$ & 7,816 & 7,385 & 5,512 & 13,328 & 12,897 & \\
\hline
\end{tabular}


Tabela 5.4 ( continuação)

\begin{tabular}{|c|c|c|c|c|c|c|c|c|}
\hline No & Soluto & Fórmula & $\alpha_{D}\left(\AA^{3}\right)$ & $\alpha_{0}\left(\AA^{3}\right)$ & $\sum \alpha_{i}\left(\AA^{3}\right)$ & $\alpha_{\text {calc (D) }}\left(\AA^{3}\right)$ & $\alpha_{\text {calc (o) }}\left(A^{3}\right)$ & $\alpha_{\exp }\left(\AA^{3}\right)$ \\
\hline$\overline{s 038}$ & 2,3,4-trimetilpentano & $\overline{\mathrm{C}_{8} \mathrm{H}_{18}}$ & 8,884 & 8,402 & 6,234 & 15,119 & 14,636 & \\
\hline s037 & 2,2,4-trimetilpentano & $\mathrm{C}_{8} \mathrm{H}_{18}$ & 8,817 & 8,343 & 6,234 & 15,051 & 14,578 & \\
\hline s032 & 2,3-dimetilpentano & $\mathrm{C}_{7} \mathrm{H}_{16}$ & 7,791 & 7,367 & 5,512 & 13,304 & 12,879 & \\
\hline s033 & 2,4-dimetilpentano & $\mathrm{C}_{7} \mathrm{H}_{16}$ & 7,783 & 7,358 & 5,512 & 13,295 & 12,871 & \\
\hline s035 & $n$-octano & $\mathrm{C}_{8} \mathrm{H}_{18}$ & 8,957 & 8,457 & 6,234 & 15,191 & 14,691 & \\
\hline s031 & 2,2-dimetilpentano & $\mathrm{C}_{7} \mathrm{H}_{16}$ & 7,741 & 7,323 & 5,512 & 13,253 & 12,835 & \\
\hline s034 & 3,3-dimetilpentano & $\mathrm{C}_{7} \mathrm{H}_{16}$ & 7,724 & 7,308 & 5,512 & 13,236 & 12,820 & \\
\hline s043 & $n$-dodecano & $\mathrm{C}_{12} \mathrm{H}_{26}$ & 13,394 & 12,645 & 9,123 & 22,517 & 21,768 & \\
\hline s027 & 2,3-dimetilbutano & $\mathrm{C}_{6} \mathrm{H}_{14}$ & 6,675 & 6,314 & 4,790 & 11,465 & 11,104 & \\
\hline s042 & $n$-undecano & $\mathrm{C}_{11} \mathrm{H}_{24}$ & 12,284 & 11,597 & 8,401 & 20,685 & 19,998 & \\
\hline s044 & $n$-tridecano & $\mathrm{C}_{13} \mathrm{H}_{28}$ & 14,504 & 13,693 & 9,845 & 24,349 & 23,537 & \\
\hline s026 & 2,2-dimetilbutano & $\mathrm{C}_{6} \mathrm{H}_{14}$ & 6,612 & 6,259 & 4,790 & 11,402 & 11,049 & \\
\hline s039 & $n$-nonano & $\mathrm{C}_{9} \mathrm{H}_{2 \mathrm{n}}$ & 10,065 & 9,503 & 6,956 & 17,021 & 16,459 & \\
\hline s041 & $n$-decano & $\mathrm{C}_{10} \mathrm{H}_{22}$ & 11,174 & 10,550 & 7,678 & 18,853 & 18,229 & \\
\hline s040 & 3,3-dimetilpentano & $\mathrm{C}_{7} \mathrm{H}_{16}$ & 7,725 & 7,309 & 5,512 & 13,237 & 12,821 & . \\
\hline s028 & $n$-heptano & $\mathrm{C}_{7} \mathrm{H}_{16}$ & 7,850 & 7,412 & 5,512 & 13,363 & 12,924 & 13,61 \\
\hline s068 & Ciclohexeno & $\mathrm{C}_{6} \mathrm{H}_{10}$ & 6,982 & 6,470 & 3,875 & 10,858 & 10,345 & \\
\hline s070 & 1,3-butadieno & $\mathrm{C}_{4} \mathrm{H}_{6}$ & 5,937 & 5,224 & 2,431 & 8,368 & 7,655 & \\
\hline s069 & 1-metilciclohexano & $\mathrm{C}_{7} \mathrm{H}_{12}$ & 8,285 & 7,649 & 4,597 & 12,883 & 12,246 & \\
\hline s065 & 1-hepteno & $\mathrm{C}_{7} \mathrm{H}_{14}$ & 8,223 & 7,674 & 5,055 & 13,278 & 12,729 & \\
\hline s016 & Etano & $\mathrm{C}_{2} \mathrm{H}_{6}$ & 2,349 & 2,216 & 1,902 & 4,251 & 4,118 & 4,48 \\
\hline s067 & 1-noneno & $\mathrm{C}_{0} \mathrm{H}_{18}$ & 10,451 & 9,775 & 6,499 & 16,950 & 16,274 & \\
\hline s015 & Metano & $\mathrm{CH}_{4}$ & 1,183 & 1,123 & 1,180 & 2,363 & 2,302 & 2,60 \\
\hline s066 & 1-Octeno & $\mathrm{C}_{8} \mathrm{H}_{16}$ & 9,337 & 8,724 & 5,777 & 15,114 & 14,501 & \\
\hline s083 & 2-Butino & $\mathrm{C}_{4} \mathrm{H}_{6}$ & 4,927 & 4,573 & 2,431 & 7,358 & 7,004 & \\
\hline s082 & 1-Butino & $\mathrm{C}_{4} \mathrm{H}_{6}$ & 4,454 & 4,177 & 2,431 & 6,885 & 6,608 & \\
\hline s081 & Propino & $\mathrm{C}_{3} \mathrm{H}_{4}$ & 3,318 & 3,106 & 1,709 & 5,027 & 4,815 & \\
\hline s080 & Etino & $\mathrm{C}_{2} \mathrm{H}_{2}$ & 1,708 & 1,745 & 0,987 & 2,695 & 2,732 & 3,33 \\
\hline s079 & 1,4-Ciclohexadieno & $\mathrm{C}_{6} \mathrm{H}_{8}$ & 7,226 & 6,620 & 3,418 & 10,644 & 10,038 & \\
\hline s078 & 1,3-Ciclohexadieno & $\mathrm{C}_{6} \mathrm{H}_{8}$ & 7,513 & 6,722 & 3,418 & 10,931 & 10,140 & \\
\hline s077 & 1,3,5-Cicloheptatrieno & $\mathrm{C}_{7} \mathrm{H}_{8}$ & 8,851 & 7,940 & 3,683 & 12,534 & 11,622 & \\
\hline s084 & 1-Pentino & $\mathrm{C}_{5} \mathrm{H}_{8}$ & 5,622 & 5,275 & 3,153 & 8,775 & 8,428 & \\
\hline s076 & 1,5-Ciclooctadieno & $\mathrm{C}_{n} \mathrm{H}_{12}$ & 9,043 & 8,402 & 4,862 & 13,905 & 13,264 & \\
\hline s085 & 1-Hexino & $\mathrm{C}_{6} \mathrm{H}_{10}$ & 6,745 & 6,334 & 3,875 & 10,620 & 10,209 & \\
\hline s075 & 1,5-Hexadieno & $\mathrm{C}_{6} \mathrm{H}_{10}$ & 7,470 & 6,872 & 3,875 & 11,345 & 10,747 & \\
\hline s074 & 1,4-Hexadieno & $\mathrm{C}_{62} \mathrm{H}_{10}$ & 7,824 & 7,124 & 3,875 & 11,699 & 11,000 & \\
\hline s088 & 1-Nonino & $\mathrm{C}_{0} \mathrm{H}_{16}$ & 10,109 & 9,507 & 6,042 & 16,151 & 15,549 & \\
\hline s087 & 1-Octino & $\mathrm{C}_{8} \mathrm{H}_{14}$ & 8,992 & 8,453 & 5,319 & 14,311 & 13,772 & \\
\hline s086 & 1-Heptino & $\mathrm{C}_{7} \mathrm{H}_{12}$ & 7,868 & 7,393 & 4,597 & 12,466 & 11,990 & \\
\hline s071 & 2-metil-1,3-butadieno & $\mathrm{C}_{5} \mathrm{H}_{8}$ & 6,933 & 6,174 & 3,153 & 10,086 & 9,328 & \\
\hline s073 & 1,4-Pentadieno & $\mathrm{C}_{5} \mathrm{H}_{8}$ & 6,320 & 5,789 & $3,1,53$ & 9,473 & 8,942 & \\
\hline s072 & 2,3-dimetil-1,3-butadieno & $\mathrm{C}_{6} \mathrm{H}_{10}$ & 7,506 & 6,863 & 3,875 & 11,382 & 10,738 & \\
\hline s062 & 2-metil-2-buteno & $\mathrm{C}_{5} \mathrm{H}_{10}$ & 6,238 & 5,714 & 3,611 & 9,848 & 9,325 & \\
\hline s059 & 1-Penteno & $\mathrm{C}_{5} \mathrm{H}_{10}$ & 5,980 & 5,561 & 3,611 & 9,591 & 9,171 & \\
\hline s019 & 2-metilpropano & $\mathrm{C}_{4} \mathrm{H}_{10}$ & 4,482 & 4,238 & 3,346 & 7,828 & 7,584 & \\
\hline s017 & Propano & $\mathrm{C}_{3} \mathrm{H}_{8}$ & 3,444 & 3,251 & 2,624 & 6,067 & 5,875 & 6,38 \\
\hline s060 & cis-2-penteno & $\mathrm{C}_{5} \mathrm{H}_{10}$ & 6,234 & 5,749 & 3,611 & 9,845 & 9,360 & \\
\hline s018 & $n$-butano & $\mathrm{C}_{4} \mathrm{H}_{10}$ & 4,541 & 4,287 & 3,346 & 7,887 & 7,633 & \\
\hline s061 & 3-metil1-buteno & $\mathrm{C}_{5} \mathrm{H}_{10}$ & 5,891 & 5,492 & 3,611 & 9,501 & 9,102 & \\
\hline
\end{tabular}


Tabela 5.4 ( continuação)

\begin{tabular}{|c|c|c|c|c|c|c|c|c|}
\hline No & Soluto & Fórmula & $\alpha_{D}\left(\AA^{3}\right)$ & $a_{0}\left(\AA^{3}\right)$ & $\Sigma \alpha_{i}\left(A^{3}\right)$ & $\alpha_{\text {calc (D) }}\left(A^{3}\right)$ & $\alpha_{\text {calc (o) }}\left(\AA^{3}\right)$ & $\alpha_{\text {exp }}\left(\AA^{3}\right)$ \\
\hline s058 & Isobuteno & $\mathrm{C}_{4} \mathrm{H}_{8}$ & 4,940 & 4,552 & 2.888 & 7,828 & 7,440 & \\
\hline s064 & 2-metil-1-penteno & $\mathrm{C}_{6} \mathrm{H}_{12}$ & 7,181 & 6,669 & 4,333 & 11,514 & 11,002 & \\
\hline s063 & 1-Нехело & $\mathrm{C}_{6} \mathrm{H}_{12}$ & 7,104 & 6,620 & 4,333 & 11,437 & 10,952 & \\
\hline \multicolumn{9}{|c|}{ Amidas } \\
\hline s573 & $N, N$-dietilbenzamida & $\mathrm{C}_{11} \mathrm{H}_{15} \mathrm{NO}$ & 15,384 & 14,073 & 6,933 & 22,317 & 21,006 & \\
\hline s572 & $N, N$-dimetilbenzamida & $\mathrm{C}_{9} \mathrm{H}_{11} \mathrm{NO}$ & 13,126 & 11,938 & 5,489 & 18,615 & 17,427 & \\
\hline s571 & $n$-metilbenzamida & $\mathrm{C}_{8} \mathrm{H}_{9} \mathrm{NO}$ & 11,859 & 10,765 & 4,767 & 16,625 & 15,532 & \\
\hline s570 & 3-metilbenzamida & $\mathrm{C}_{8} \mathrm{H}_{9} \mathrm{NO}$ & 12,131 & 10,940 & 4,767 & 16,898 & 15,707 & \\
\hline s569 & Benzamida & $\mathrm{C}_{7} \mathrm{H}_{7} \mathrm{NO}$ & 10,741 & 9,664 & 4,045 & 14,786 & 13,708 & \\
\hline s299 & $N, N$-dimetilpropanamida & $\mathrm{C}_{5} \mathrm{H}_{11} \mathrm{NO}$ & 7,480 & 6,953 & 4,430 & 11,910 & 11,383 & \\
\hline s297 & $N, N$-dimetilacetamida & $\mathrm{C}_{4} \mathrm{H}_{9} \mathrm{NO}$ & 6,382 & 5,922 & 3,708 & 10,090 & 9,630 & \\
\hline s290 & Propionamida & $\mathrm{C}_{3} \mathrm{H}_{7} \mathrm{NO}$ & 5,052 & 4,695 & 2,986 & 8,038 & 7,681 & \\
\hline s291 & Butanamida & $\mathrm{C}_{4} \mathrm{H}_{9} \mathrm{NO}$ & 6,171 & 5,751 & 3,708 & 9,879 & 9,459 & \\
\hline s292 & Isobutanamida & $\mathrm{C}_{4} \mathrm{H}_{9} \mathrm{NO}$ & 6,112 & 5,700 & 3,708 & 9,820 & 9,408 & \\
\hline s298 & $N, N$-dietilacetamida & $\mathrm{C}_{6} \mathrm{H}_{13} \mathrm{NO}$ & 8,670 & 8,082 & 5,152 & 13,822 & 13,234 & \\
\hline s293 & $n$-metilformamida & $\mathrm{C}_{2} \mathrm{H}_{5} \mathrm{NO}$ & 4,159 & 3,818 & 2,264 & 6,423 & 6,082 & \\
\hline s294 & $n$-metilacetamida & $\mathrm{C}_{3} \mathrm{H}_{7} \mathrm{NO}$ & 5,174 & 4,795 & 2.986 & 8,160 & 7,780 & \\
\hline s295 & $n$-butilacetamida & $\mathrm{C}_{6} \mathrm{H}_{13} \mathrm{NO}$ & 8,610 & 8,025 & 5,152 & 13,762 & 13,177 & \\
\hline s296 & $N, N$-dimetilformamida & $\mathrm{C}_{3} \mathrm{H}_{7} \mathrm{NO}$ & 5,300 & 4,891 & 2,986 & 8,285 & 7,877 & \\
\hline s 288 & Formamida & $\mathrm{CH}_{3} \mathrm{NO}$ & 2,881 & 2,636 & 1,542 & 4,422 & 4,178 & 7,77 \\
\hline s289 & Acetamida & $\mathrm{C}_{2} \mathrm{H}_{5} \mathrm{NO}$ & 3,957 & 3,661 & 2,264 & 6,221 & 5,924 & \\
\hline s577 & 2-metilacetanilida & $\mathrm{C}_{2} \mathrm{H}_{11} \mathrm{NO}$ & 13,462 & 12,135 & 5,489 & 18,951 & 17,624 & \\
\hline s578 & 3-metilacetanilida & $\mathrm{C}_{9} \mathrm{H}_{11} \mathrm{NO}$ & 14,279 & 12,670 & 5,489 & 19,768 & 18,158 & \\
\hline s579 & 4-metilacetanilida & $\mathrm{C}_{9} \mathrm{H}_{11} \mathrm{NO}$ & 14,591 & 12,828 & 5,489 & 20,079 & 18,317 & \\
\hline s576 & Acetanilida & $\mathrm{C}_{8} \mathrm{H}_{9} \mathrm{NO}$ & 12,534 & 11,166 & 4,767 & 17,300 & 15,933 & \\
\hline s575 & 4-metilformanilida & $\mathrm{C}_{8} \mathrm{H}_{9} \mathrm{NO}$ & 14,272 & 12,033 & 4,767 & 19,039 & 16,800 & \\
\hline s574 & Formanilida & $\mathrm{C}_{7} \mathrm{H}_{7} \mathrm{NO}$ & 12,205 & 10,601 & 4,045 & 16,250 & 14,646 & \\
\hline \multicolumn{9}{|c|}{ Aminas $e$ Nitroalcanos } \\
\hline s257 & Metilamina & $\mathrm{CH}_{5} \mathrm{~N}$ & 2,139 & 2,008 & 1,767 & 3,905 & 3,775 & \\
\hline s562 & $N, N$-dimetilbenzilamina & $\mathrm{C}_{9} \mathrm{H}_{13} \mathrm{~N}$ & 12,238 & 11,211 & 5,714 & 17,952 & 16,925 & \\
\hline s561 & Benzilamina & $\mathrm{C}_{7} \mathrm{H}_{9} \mathrm{~N}$ & 9,698 & 8,845 & 4.270 & 13,968 & 13,114 & \\
\hline s258 & Etilamina & $\mathrm{C}_{2} \mathrm{H}_{7} \mathrm{~N}$ & 3,254 & 3,065 & 2,489 & 5,743 & 5,553 & 5,65 \\
\hline s266 & $n$-hexilamina & $\mathrm{C}_{6} \mathrm{H}_{15} \mathrm{~N}$ & 7,684 & 7,244 & 5,377 & 13,062 & 12,621 & \\
\hline s267 & $n$-heptilamina & $\mathrm{C}_{7} \mathrm{H}_{77} \mathrm{~N}$ & 8,791 & 8,288 & 6.099 & 14,890 & 14,388 & \\
\hline s268 & $n$-octilamina & $\mathrm{C}_{8} \mathrm{H}_{19} \mathrm{~N}$ & 9,900 & 9,335 & 6,821 & 16,721 & 16,156 & \\
\hline s270 & Alilamina & $\mathrm{C}_{3} \mathrm{H}_{7} \mathrm{~N}$ & 4,654 & 4,306 & 2.753 & 7,408 & 7,059 & \\
\hline s271 & Ciclohexilamina & $\mathrm{C}_{6} \mathrm{H}_{13} \mathrm{~N}$ & 7,474 & 7,051 & 4,920 & 12,394 & 11,970 & \\
\hline s272 & Dimetilamina & $\mathrm{C}_{2} \mathrm{H}_{7} \mathrm{~N}$ & 3,327 & 3,127 & 2,489 & 5,816 & 5,615 & 5,87 \\
\hline s269 & n-nonilamina & $\mathrm{C}_{9} \mathrm{H}_{21} \mathrm{~N}$ & 11,009 & 10,382 & 7,543 & 18,552 & 17,925 & \\
\hline s259 & $n$-propilamina & $\mathrm{C}_{3} \mathrm{H}_{9} \mathrm{~N}$ & 4,359 & 4,106 & 3,211 & 7,570 & 7,317 & \\
\hline s261 & $n$-butilamina & $\mathrm{C}_{4} \mathrm{H}_{11} \mathrm{~N}$ & 5,470 & 5,154 & 3,933 & 9,403 & 9,087 & 9,66 \\
\hline s262 & Isobutilamina & $\mathrm{C}_{4} \mathrm{H}_{11} \mathrm{~N}$ & 5,412 & 5,104 & 3,933 & 9,344 & 9,037 & \\
\hline s263 & $s e c$-butilamina & $\mathrm{C}_{4} \mathrm{H}_{11} \mathrm{~N}$ & 5,421 & 5,116 & 3,933 & 9,354 & 9,049 & \\
\hline s264 & tert-butilamina & $\mathrm{C}_{4} \mathrm{H}_{11} \mathrm{~N}$ & 5,334 & 5,045 & 3,933 & 9,267 & 8,978 & \\
\hline s265 & $n$-pentilamina & $\mathrm{C}_{5} \mathrm{H}_{13} \mathrm{~N}$ & 6,579 & 6,200 & 4,655 & 11,234 & 10,855 & \\
\hline s260 & Isopropilamina & $\mathrm{C}_{3} \mathrm{H}_{9} \mathrm{~N}$ & 4,327 & 4,082 & 3,211 & 7,538 & 7,292 & \\
\hline s282 & 1-nitropropano & $\mathrm{C}_{3} \mathrm{H}_{7} \mathrm{NO}_{2}$ & 5,435 & 5,080 & 3,218 & 8,653 & 8,298 & \\
\hline s283 & 2-nitropropano & $\mathrm{C}_{3} \mathrm{H}_{7} \mathrm{NO}_{2}$ & 5,423 & 5,068 & 3,218 & 8,641 & 8,287 & \\
\hline s284 & 1-nitrobutano & $\mathrm{C}_{4} \mathrm{H}_{9} \mathrm{NO}_{2}$ & 6,539 & 6,122 & 3,940 & 10,479 & 10,062 & \\
\hline
\end{tabular}


Tabela 5.4 ( continuação)

\begin{tabular}{|c|c|c|c|c|c|c|c|c|}
\hline No & Soluto & Fórmula & $\alpha_{D}\left(\AA^{3}\right)$ & $\alpha_{0}\left(\AA^{3}\right)$ & $\sum \alpha_{i}\left(\AA^{3}\right)$ & $\alpha_{\text {calc (D) }}\left(\AA^{3}\right)$ & $\alpha_{\text {calc (o) }}\left(A^{3}\right)$ & $\alpha_{\text {exp }}\left(\AA^{3}\right)$ \\
\hline s285 & 2-metil-2-nitropropano & $\mathrm{C}_{4} \mathrm{H}_{9} \mathrm{NO}_{2}$ & 6,473 & 6,065 & 3,940 & 10,413 & 10,005 & \\
\hline s286 & 1-nitropentano & $\mathrm{C}_{5} \mathrm{H}_{11} \mathrm{NO}_{2}$ & 7,649 & 7,169 & 4,662 & 12,311 & 11,831 & \\
\hline s287 & 1-nitrohexano & $\mathrm{C}_{6} \mathrm{H}_{13} \mathrm{NO}_{2}$ & 8,756 & 8,214 & 5,385 & 14,141 & 13,599 & \\
\hline s278 & Trietilamina & $\mathrm{C}_{6} \mathrm{H}_{15} \mathrm{~N}$ & 7,925 & 7,469 & 5,377 & 13,302 & 12,846 & \\
\hline s274 & Di-n-propilamina & $\mathrm{C}_{6} \mathrm{H}_{15} \mathrm{~N}$ & 7,831 & 7,378 & 5,377 & 13,208 & 12,755 & \\
\hline s275 & Di-isopropilamina & $\mathrm{C}_{6} \mathrm{H}_{15} \mathrm{~N}$ & 7,754 & 7,319 & 5,377 & 13,131 & 12,696 & \\
\hline s276 & Di-n-butilamina & $\mathrm{C}_{8} \mathrm{H}_{19} \mathrm{~N}$ & 10,059 & 9,481 & 6,821 & 16,880 & 16,302 & \\
\hline s277 & Trimetilamina & $\mathrm{C}_{3} \mathrm{H}_{9} \mathrm{~N}$ & 4,483 & 4,219 & 3,211 & 7,694 & 7,430 & \\
\hline $\mathrm{s} 281$ & Nitroetano & $\mathrm{C}_{2} \mathrm{H}_{5} \mathrm{NO}_{2}$ & 4,321 & 4,028 & 2,496 & 6,817 & 6,524 & \\
\hline s 280 & Nitrometano & $\mathrm{CH}_{3} \mathrm{NO}_{2}$ & 3,186 & 2,962 & 1,774 & 4,960 & 4,736 & \\
\hline s273 & Dietilamina & $\mathrm{C}_{4} \mathrm{H}_{11} \mathrm{~N}$ & 5,609 & 5,281 & 3,933 & 9,542 & 9,213 & \\
\hline \multicolumn{9}{|c|}{ Hidrocarbonetos Aromáticos e Derivados } \\
\hline s489 & Iodobenzeno & $\mathrm{C}_{6} \mathrm{H}_{5} \mathrm{I}$ & 10,616 & 9,372 & 6,825 & 17,441 & 16,196 & \\
\hline s494 & 3-metilanisol & $\mathrm{C}_{8} \mathrm{H}_{10} \mathrm{O}$ & 11,150 & 10,075 & 4,637 & 15,787 & 14,712 & \\
\hline s487 & 4-bromotolueno & $\mathrm{C}_{7} \mathrm{H}_{7} \mathrm{Br}$ & 11,017 & 9,868 & 5,712 & 16,729 & 15,581 & \\
\hline s495 & 4-metilanisol & $\mathrm{C}_{8} \mathrm{H}_{10} \mathrm{O}$ & 11,353 & 10,193 & 4,637 & 15,990 & 14,830 & \\
\hline s486 & 3-bromotolueno & $\mathrm{C}_{7} \mathrm{H}_{7} \mathrm{Br}$ & 10,817 & 9,842 & 5,712 & 16,529 & 15,555 & \\
\hline s485 & 2-bromotolueno & $\mathrm{C}_{7} \mathrm{H}_{7} \mathrm{Br}$ & 10,810 & 9,716 & 5,712 & 16,522 & 15,428 & \\
\hline s 484 & 1,4-dibromobenzeno & $\mathrm{C}_{6} \mathrm{H}_{4} \mathrm{Br}_{2}$ & 11,881 & 10,523 & 7,020 & 18,901 & 17,543 & \\
\hline s483 & 1,3-dibromobenzeno & $\mathrm{C}_{6} \mathrm{H}_{4} \mathrm{Br}_{2}$ & 11,770 & 10,443 & 7,020 & 18,790 & 17,462 & \\
\hline s482 & 1,2-dibromobenzeno & $\mathrm{C}_{6} \mathrm{H}_{4} \mathrm{Br}_{2}$ & 11,240 & 10,026 & 7,020 & 18,259 & 17,046 & \\
\hline s 481 & Bromobenzeno & $\mathrm{C}_{6} \mathrm{H}_{5} \mathrm{Br}$ & 9,499 & 8,531 & 4,990 & 14,489 & 13,521 & \\
\hline $\mathrm{s} 480$ & Cloreto de benzila & $\mathrm{C}_{7} \mathrm{H}_{7} \mathrm{Cl}$ & 10,375 & 9,349 & 4,777 & 15,153 & 14,126 & \\
\hline s510 & 3-cloroacetofenona & $\mathrm{C}_{8} \mathrm{H}_{7} \mathrm{OCl}$ & 12,217 & 11,019 & 5,275 & 17,491 & 16,294 & \\
\hline s 488 & Brometo de benzila & $\mathrm{C}_{7} \mathrm{H}_{7} \mathrm{Br}$ & 11,724 & 10,236 & 5,712 & 17,436 & 15,948 & \\
\hline s509 & 4-fluoracetofenona & $\mathrm{C}_{8} \mathrm{H}_{7} \mathrm{OF}$ & 10,790 & 9,791 & 4,149 & 14,939 & 13,940 & \\
\hline s508 & 4-metilacetofenona & $\mathrm{C}_{9} \mathrm{H}_{10} \mathrm{O}$ & 12,012 & 10,898 & 4,902 & 16,914 & 15,800 & \\
\hline s507 & 3-metilacetofenona & $\mathrm{C}_{9} \mathrm{H}_{10} \mathrm{O}$ & 11,873 & 10,807 & 4,902 & 16,775 & 15,709 & \\
\hline s506 & Acetofenona & $\mathrm{C}_{8} \mathrm{H}_{8} \mathrm{O}$ & 10,625 & 9,657 & 4,180 & 14,804 & 13,837 & \\
\hline s493 & 2-metilanisol & $\mathrm{C}_{8} \mathrm{H}_{10} \mathrm{O}$ & 10,906 & 9,923 & 4,637 & 15,543 & 14,560 & \\
\hline s479 & 4-clorotolueno & $\mathrm{C}_{7} \mathrm{H}_{7} \mathrm{Cl}$ & 10,633 & 9,511 & 4,777 & 15,411 & 14,289 & \\
\hline s500 & 1,4-dimetoxibenzeno & $\mathrm{C}_{8} \mathrm{H}_{10} \mathrm{O}_{2}$ & 12,404 & 11,056 & 4,869 & 17,273 & 15,925 & \\
\hline s 499 & 1,3-dimetoxibenzeno & $\mathrm{C}_{8} \mathrm{H}_{30} \mathrm{O}_{2}$ & 12,175 & 10,986 & 4,869 & 17,045 & 15,855 & \\
\hline s 498 & Eter difenílico & $\mathrm{C}_{12} \mathrm{H}_{10} \mathrm{O}$ & 17,480 & 15,515 & 5,696 & 23,176 & 21,211 & \\
\hline s497 & 4-cloroanisol & $\mathrm{C}_{7} \mathrm{H}_{7} \mathrm{OCl}$ & 11,696 & 10,392 & 5,010 & 16,706 & 15,402 & \\
\hline$s 496$ & 2,6-dimetilanisol & $\mathrm{C}_{9} \mathrm{H}_{12} \mathrm{O}$ & 12,232 & 11,119 & 5,359 & 17,591 & 16,478 & \\
\hline $\mathrm{s} 455$ & Naftaceno & $\mathrm{C}_{18} \mathrm{H}_{12}$ & 36,563 & 27,731 & 7,509 & 44,072 & 35,240 & \\
\hline$s 461$ & Benzo[ghl]perileno & $\mathrm{C}_{22} \mathrm{H}_{12}$ & 37,522 & 30,713 & 8,568 & 46,090 & 39,281 & \\
\hline$s 460$ & Benzo[a]pireno & $\mathrm{C}_{20} \mathrm{H}_{12}$ & 37,720 & 29,378 & 8,038 & 45,758 & 37,417 & \\
\hline$s 459$ & Dibenzo[c]antraceno & $\mathrm{C}_{22} \mathrm{H}_{14}$ & 46,887 & 33,324 & 9,025 & 55,913 & 42,349 & \\
\hline $\mathrm{s} 458$ & 3-methilcolantreno & $\mathrm{C}_{21} \mathrm{H}_{14}$ & 106,618 & 30,658 & 8,761 & 115,379 & 39,418 & \\
\hline $\mathrm{s} 457$ & Perileno & $\mathrm{C}_{20} \mathrm{H}_{12}$ & 37,861 & 28,367 & 8,038 & 45,899 & 36,405 & \\
\hline$s 463$ & Fluorbenzeno & $\mathrm{C}_{6} \mathrm{H}_{5} \mathrm{~F}$ & 7,674 & 6,957 & 2,930 & 10,604 & 9,887 & \\
\hline s 454 & benzo[a]antraceno & $\mathrm{C}_{18} \mathrm{H}_{12}$ & 31,069 & 25,652 & 7,509 & 38,578 & 33,161 & \\
\hline$s 453$ & Pireno & $\mathrm{C}_{16} \mathrm{H}_{10}$ & 28,182 & 21,514 & 6,522 & 34,704 & 28,036 & \\
\hline $\mathrm{s} 452$ & benzo[b]fluoreno & $\mathrm{C}_{17} \mathrm{H}_{12}$ & 27,378 & 22,828 & 7,244 & 34,622 & 30,073 & \\
\hline $\mathrm{s} 451$ & benzo[a]fluoreno & $\mathrm{C}_{17} \mathrm{H}_{12}$ & 26,090 & 22,502 & 7,244 & 33,334 & 29,746 & \\
\hline$\$ 450$ & Fluorantreno & $\mathrm{C}_{16} \mathrm{H}_{10}$ & 25,229 & 21,254 & 6,522 & 31,752 & 27,777 & \\
\hline s449 & 3-metilfenantreno & $\mathrm{C}_{15} \mathrm{H}_{12}$ & 22,972 & 19,777 & 6,715 & 29,687 & 26,492 & \\
\hline
\end{tabular}


Tabela 5.4 (continuação)

\begin{tabular}{|c|c|c|c|c|c|c|c|c|}
\hline No & Soluto & Fórmula & $\alpha_{D}\left(\AA^{3}\right)$ & $\alpha_{0}\left(A^{3}\right)$ & $\sum \alpha_{i}\left(\AA^{\prime}\right)$ & $\alpha_{\text {calc (D) }}\left(A^{3}\right)$ & $\alpha_{\text {calc (o) }}\left(\AA^{3}\right)$ & $\alpha_{\exp }\left(\AA^{5}\right)$ \\
\hline$\overline{s 456}$ & Criseno & $\mathrm{C}_{18} \mathrm{H}_{12}$ & 29,635 & 24,910 & 7.509 & 37,144 & 32,419 & \\
\hline s478 & 3-clorotolueno & $\mathrm{C}_{7} \mathrm{H}_{7} \mathrm{Cl}$ & 10,514 & 9,448 & 4,777 & 15,291 & 14,225 & \\
\hline$s 477$ & 2-clorotolueno & $\mathrm{C}_{7} \mathrm{H}_{7} \mathrm{Cl}$ & 10,410 & 9,365 & 4,777 & 15,188 & 14,142 & \\
\hline$s 476$ & Hexaclorobenzeno & $\mathrm{C}_{6} \mathrm{Cl}_{6}$ & 19,030 & 16,102 & 9,530 & 28,560 & 25,632 & \\
\hline$\$ 475$ & Pentaclorobenzeno & $\mathrm{C}_{6} \mathrm{HCl}_{5}$ & 16,892 & 14,443 & 8,435 & 25,327 & 22,878 & \\
\hline$s 474$ & 1,2,4,5-tetraclorobenzeno & $\mathrm{C}_{6} \mathrm{H}_{2} \mathrm{Cl}_{4}$ & 15,017 & 12,927 & 7,340 & 22,357 & 20,267 & \\
\hline s473 & 1,2,3,5-tetraclorobenzeno & $\mathrm{C}_{6} \mathrm{H}_{2} \mathrm{Cl}_{4}$ & 14,841 & 12,849 & 7,340 & 22,181 & 20,189 & \\
\hline$s 462$ & $p$-terfenilo & $\mathrm{C}_{18} \mathrm{H}_{14}$ & 30,225 & 24,844 & 7,966 & 38,192 & 32,810 & \\
\hline $\mathrm{s} 471$ & 1,3,5-triclorobenzeno & $\mathrm{C}_{6} \mathrm{H}_{3} \mathrm{Cl}_{3}$ & 12,903 & 11,336 & 6,245 & 19,148 & 17,581 & \\
\hline s470 & 1,2,4-triclorobenzeno & $\mathrm{C}_{6} \mathrm{H}_{3} \mathrm{Cl}_{3}$ & 12,902 & 11,269 & 6,245 & 19,147 & 17,514 & \\
\hline$s 469$ & 1,2,3-triclorobenzeno & $\mathrm{C}_{6} \mathrm{H}_{3} \mathrm{Cl}_{3}$ & 12,561 & 11,040 & 6,245 & 18,806 & 17,285 & \\
\hline$s 468$ & 1,4-diclorobenzeno & $\mathrm{C}_{6} \mathrm{H}_{4} \mathrm{Cl}_{2}$ & 11,077 & 9,776 & 5,150 & 16,227 & 14,926 & \\
\hline s467 & 1,3-diclorobenzeno & $\mathrm{C}_{6} \mathrm{H}_{4} \mathrm{Cl}_{2}$ & 10,921 & 9,698 & 5,150 & 16,071 & 14,849 & \\
\hline s466 & 1,2-diclorobenzeno & $\mathrm{C}_{6} \mathrm{H}_{4} \mathrm{Cl}_{2}$ & 10,758 & 9,554 & 5,150 & 15,908 & 14,704 & \\
\hline$s 465$ & Clorobenzeno & $\mathrm{C}_{6} \mathrm{H}_{5} \mathrm{Cl}$ & 9,090 & 8,170 & 4,055 & 13,146 & 12,226 & \\
\hline s464 & Trifluormetil benzeno & $\mathrm{C}_{7} \mathrm{H}_{5} \mathrm{~F}_{3}$ & 8,779 & 8,031 & 3,591 & 12,370 & 11,622 & \\
\hline$s 472$ & 1,2,3,4-tetraclorobenzeno & $\mathrm{C}_{6} \mathrm{H}_{2} \mathrm{Cl}_{4}$ & 14,654 & 12,693 & 7,340 & 21,994 & 20,033 & \\
\hline s565 & 2-nitrotolueno & $\mathrm{C}_{7} \mathrm{H}_{7} \mathrm{NO}_{2}$ & 11,282 & 10,162 & 4,277 & 15,559 & 14,439 & \\
\hline s568 & 1-cloro-4-nitrobenzeno & $\mathrm{C}_{6} \mathrm{H}_{4} \mathrm{NO}_{2} \mathrm{Cl}$ & 12,071 & 10,641 & 4,650 & 16,721 & 15,291 & \\
\hline s567 & 4-nitrotolueno & $\mathrm{C}_{7} \mathrm{H}_{7} \mathrm{NO}_{2}$ & 11,506 & 10,279 & 4,277 & 15,783 & 14,556 & \\
\hline s564 & Nitrobenzeno & $\mathrm{C}_{6} \mathrm{H}_{5} \mathrm{NO}_{2}$ & 9,832 & 8,879 & 3,555 & 13,387 & 12,434 & 12,92 \\
\hline s563 & Azobenzeno & $\mathrm{C}_{12} \mathrm{H}_{10} \mathrm{~N}_{2}$ & 22,221 & 18,706 & 6,180 & 28,402 & 24,887 & \\
\hline s560 & 4-aminobifenil & $\mathrm{C}_{12} \mathrm{H}_{11} \mathrm{~N}$ & 20,867 & 17,293 & 6,051 & 26,917 & 23,343 & \\
\hline s559 & 3-aminobifenil & $\mathrm{C}_{12} \mathrm{H}_{11} \mathrm{~N}$ & 19,391 & 16,683 & 6.051 & 25,442 & 22,734 & \\
\hline s566 & 3-nitrotolueno & $\mathrm{C}_{7} \mathrm{H}_{7} \mathrm{NO}_{2}$ & 11,274 & 10,161 & 4,277 & 15,551 & 14,438 & \\
\hline s519 & benzoato de fenila & $\mathrm{C}_{13} \mathrm{H}_{10} \mathrm{O}_{2}$ & 18,941 & 16,971 & 6,193 & 25,133 & 23,164 & \\
\hline s518 & Benzoato de $n$-butila & $\mathrm{C}_{11} \mathrm{H}_{14} \mathrm{O}_{2}$ & 14,924 & 13,697 & 6,578 & 21,502 & 20,276 & \\
\hline s517 & Benzoato de isopropila & $\mathrm{C}_{10} \mathrm{H}_{12} \mathrm{O}_{2}$ & 13,717 & 12,574 & 5,856 & 19,574 & 18,430 & \\
\hline s516 & Benzoato de $n$-propila & $\mathrm{C}_{10} \mathrm{H}_{12} \mathrm{O}_{2}$ & 13,777 & 12,619 & 5,856 & 19,633 & 18,476 & \\
\hline s515 & Benzoato de etila & $\mathrm{C}_{0} \mathrm{H}_{10} \mathrm{O}_{2}$ & 12,626 & 11,538 & 5,134 & 17,761 & 16,672 & \\
\hline s514 & Benzoato de metila & $\mathrm{C}_{8} \mathrm{H}_{8} \mathrm{O}_{2}$ & 11,381 & 10,370 & 4,412 & 15,793 & 14,782 & \\
\hline $\mathrm{s} 513$ & Benzofenona & $\mathrm{C}_{13} \mathrm{H}_{10} \mathrm{O}$ & 17,324 & 15,642 & 5,961 & 23,284 & 21,602 & \\
\hline $\mathrm{s} 512$ & Etil fenil cetona & $\mathrm{C}_{9} \mathrm{H}_{30} \mathrm{O}$ & 11,816 & 10,768 & 4,902 & 16,718 & 15,670 & \\
\hline s511 & 4-cloroacetofenona & $\mathrm{C}_{8} \mathrm{H}_{7} \mathrm{OCl}$ & 12,657 & 11,298 & 5.275 & 17,931 & 16,572 & \\
\hline s534 & 3-fluornitrobenzeno & $\mathrm{C}_{6} \mathrm{H}_{4} \mathrm{NO}_{2} \mathrm{~F}$ & 10,113 & 9,093 & 3,524 & 13,637 & 12,617 & \\
\hline s448 & 2-metilfenantreno & $\mathrm{C}_{15} \mathrm{H}_{12}$ & 23,042 & 19,832 & 6,715 & 29,757 & 26,547 & \\
\hline$s 422$ & 4-metilbifenilo & $\mathrm{C}_{13} \mathrm{H}_{12}$ & 19,310 & 16,660 & 6,186 & 25,495 & 22,846 & \\
\hline s421 & Bifenilo & $\mathrm{C}_{12} \mathrm{H}_{10}$ & 17,356 & 15,113 & 5,463 & 22,819 & 20,576 & \\
\hline$s 420$ & 1,2-difeniletano & $\mathrm{C}_{14} \mathrm{H}_{14}$ & 18,158 & 16,414 & 6,908 & 25,066 & 23,322 & \\
\hline s419 & Difenilmethano & $\mathrm{C}_{13} \mathrm{H}_{12}$ & 16,643 & 15,050 & 6,186 & 22,829 & 21,235 & \\
\hline s418 & Alilbenzeno & $\mathrm{C}_{9} \mathrm{H}_{8}$ & 11,098 & 10,169 & 4,212 & 15,310 & 14,381 & \\
\hline s417 & $\alpha$-metilestireno & $\mathrm{C}_{9} \mathrm{H}_{10}$ & 11,751 & 10,595 & 4,669 & 16,421 & 15,264 & \\
\hline$s 446$ & Fenantreno & $\mathrm{C}_{14} \mathrm{H}_{10}$ & 21,241 & 18,312 & 5,993 & 27,234 & 24,305 & \\
\hline s445 & 9,10 -dimetilantraceno & $\mathrm{C}_{16} \mathrm{H}_{14}$ & 27,243 & 22,124 & 7,437 & 34,680 & 29,561 & \\
\hline s444 & 9-metilantraceno & $\mathrm{C}_{15} \mathrm{H}_{12}$ & 25,336 & 20,805 & 6,715 & 32,051 & 27,520 & \\
\hline$s 443$ & Antraceno & $\mathrm{C}_{14} \mathrm{H}_{10}$ & 23,513 & 19,471 & 5,993 & 29,505 & 25,464 & \\
\hline$s 442$ & Azuleno & $\mathrm{C}_{10} \mathrm{H}_{8}$ & 28,815 & 14,055 & 4,477 & 33,291 & 18,532 & \\
\hline$s 441$ & 1-metilfluoreno & $\mathrm{C}_{14} \mathrm{H}_{12}$ & 19,800 & 17,356 & 6,450 & 26,250 & 23,806 & \\
\hline$s 440$ & Fluoreno & $\mathrm{C}_{13} \mathrm{H}_{10}$ & 18,438 & 16,122 & 5,728 & 24,166 & 21,850 & \\
\hline
\end{tabular}


Tabela 5.4 (continuação)

\begin{tabular}{|c|c|c|c|c|c|c|c|c|}
\hline No & Soluto & Fórmula & $\alpha_{D}\left(A^{3}\right)$ & $\alpha_{0}\left(\AA^{3}\right)$ & $\overline{\Sigma \alpha_{i}\left(\AA^{3}\right)}$ & $\alpha_{\text {calc (D) }}\left(\AA^{3}\right)$ & $\alpha_{\text {calc (o) }}\left(\AA^{3}\right)$ & $\alpha_{\text {exp }}\left(\AA^{3}\right)$ \\
\hline$\$ 415$ & Feniletino & $\overline{\mathrm{C}_{8} \mathrm{H}_{6}}$ & 11,010 & 9,807 & 3,490 & 14,500 & 13,297 & \\
\hline$s 438$ & Indano & $\mathrm{C}_{9} \mathrm{H}_{10}$ & 11,043 & 10,056 & 4,669 & 15,712 & 14,725 & \\
\hline s437 & Indeno & $\mathrm{C}_{9} \mathrm{H}_{8}$ & 11,915 & 10,581 & 4,212 & 16,127 & 14,793 & \\
\hline s391 & Etilbenzeno & $\mathrm{C}_{8} \mathrm{H}_{10}$ & 9,952 & 9,086 & 4,405 & 14,357 & 13,490 & \\
\hline s399 & 1,3,5-trimetilbenzeno & $\mathrm{C}_{9} \mathrm{H}_{12}$ & 11,609 & 10,523 & 5,127 & 16,735 & 15,650 & \\
\hline s398 & 1,2,4-trimetilbenzeno & $\mathrm{C}_{0} \mathrm{H}_{12}$ & 11,645 & 10,535 & 5,127 & 16,771 & 15,662 & \\
\hline s397 & 1,2,3-trimetilbenzeno & $\mathrm{C}_{9} \mathrm{H}_{12}$ & 11,532 & 10,466 & 5,127 & 16,659 & 15,593 & \\
\hline s396 & Isopropilbenzeno & $\mathrm{C}_{9} \mathrm{H}_{12}$ & 10,987 & 10,075 & 5,127 & 16,114 & 15,202 & \\
\hline s395 & $n$-propilbenzeno & $\mathrm{C}_{9} \mathrm{H}_{12}$ & 11,145 & 10,199 & 5,127 & 16,272 & 15,326 & \\
\hline s394 & $p$-xileno & $\mathrm{C}_{8} \mathrm{H}_{10}$ & 10,311 & 9,322 & 4,405 & 14,716 & 13,726 & \\
\hline s393 & $m$-xileno & $\mathrm{C}_{8} \mathrm{H}_{10}$ & 10,215 & 9,267 & 4,405 & 14,619 & 13,671 & \\
\hline s416 & Estireno & $\mathrm{C}_{8} \mathrm{H}_{8}$ & 11,150 & 9,827 & 3,947 & 15,098 & 13,774 & 14,45 \\
\hline$s 400$ & 2-etiltolueno & $\mathrm{C}_{9} \mathrm{H}_{12}$ & 11,265 & 10,280 & 5,127 & 16,392 & 15,406 & \\
\hline 5390 & Tolueno & $\mathrm{C}_{7} \mathrm{H}_{8}$ & 8,816 & 8,011 & 3,683 & 12,498 & 11,693 & 12,31 \\
\hline s389 & Benzeno & $\mathrm{C}_{6} \mathrm{H}_{6}$ & 7,415 & 6,756 & 2,960 & 10,376 & 9,716 & 10,33 \\
\hline s447 & 1-metilfenantreno & $\mathrm{C}_{15} \mathrm{H}_{12}$ & 22,876 & 19,692 & 6,715 & 29,591 & 26,407 & \\
\hline s392 & $o$-xileno & $\mathrm{C}_{8} \mathrm{H}_{\text {in }}$ & 10,163 & 9,229 & 4,405 & 14,568 & 13,634 & \\
\hline$s 414$ & Ciclopropilbenzeno & $\mathrm{C}_{9} \mathrm{H}_{10}$ & 11,173 & 10,180 & 4,669 & 15,843 & 14,849 & \\
\hline s413 & $n$-decilbenzeno & $\mathrm{C}_{16} \mathrm{H}_{26}$ & 19,010 & 17,610 & 10,181 & 29,191 & 27,791 & \\
\hline s412 & $n$-octilbenzeno & $\mathrm{C}_{14} \mathrm{H}_{22}$ & 16,758 & 15,488 & 8,737 & 25,495 & 24,225 & \\
\hline$s 411$ & $n$-hexilbenzeno & $\mathrm{C}_{12} \mathrm{H}_{18}$ & 14,533 & 13,389 & 7,293 & 21,826 & 20,682 & \\
\hline$s 410$ & Pentametilbenzeno & $\mathrm{C}_{11} \mathrm{H}_{16}$ & 14,369 & 12,995 & 6,571 & 20,939 & 19,566 & \\
\hline s408 & 1,2,4,5-tetrametilbenzeno & $\mathrm{C}_{10} \mathrm{H} 14$ & 13,054 & 11,793 & 5,849 & 18,903 & 17,641 & \\
\hline$s 407$ & 1,2,3,5-tetrametilbenzeno & $\mathrm{C}_{10} \mathrm{H}_{14}$ & 12,999 & 11,764 & 5,849 & 18,848 & 17,613 & \\
\hline s406 & 1,2,3,4-tetrametilbenzeno & $\mathrm{C}_{10} \mathrm{H}_{14}$ & 12,965 & 11,740 & 5,849 & 18,814 & 17,589 & \\
\hline s405 & 4-isopropiltolueno & $\mathrm{C}_{10} \mathrm{H}_{14}$ & 12,500 & 11,402 & 5,849 & 18,349 & 17,251 & \\
\hline s404 & tert-butilbenzeno & $\mathrm{C}_{10} \mathrm{H}_{14}$ & 12,060 & 11,094 & 5,849 & 17,909 & 16,943 & \\
\hline$s 403$ & Isobutilbenzeno & $\mathrm{C}_{10} \mathrm{H}_{14}$ & 12,242 & 11,238 & 5,849 & 18,091 & 17,087 & \\
\hline $\mathrm{s} 402$ & $n$-butilbenzeno & $\mathrm{C}_{10} \mathrm{H}_{14}$ & 12,273 & 11,262 & 5,849 & 18,122 & 17,111 & \\
\hline$s 401$ & 4-etiltolueno & $\mathrm{C}_{9} \mathrm{H}_{12}$ & 11,461 & 10,407 & 5,127 & 16,588 & 15,534 & \\
\hline$s 409$ & $n$-pentilbenzeno & $\mathrm{C}_{11} \mathrm{H}_{16}$ & 13,404 & 12,327 & 6,571 & 19,975 & 18,897 & \\
\hline s672 & Furano & $\mathrm{C}_{4} \mathrm{H}_{4} \mathrm{O}$ & 5,198 & 4,754 & 2,206 & 7,404 & 6,960 & 7,23 \\
\hline$s 686$ & 3,5-dimetilpiridina & $\mathrm{C}_{7} \mathrm{H}_{9} \mathrm{~N}$ & 9,924 & 8,997 & 4,270 & 14,193 & 13,266 & \\
\hline s 685 & 3,4-dimetilpiridina & $\mathrm{C}_{7} \mathrm{H}_{9} \mathrm{~N}$ & 9,814 & 8,926 & 4,270 & 14,084 & 13,196 & \\
\hline s684 & 2,6-dimetilpiridina & $\mathrm{C}_{7} \mathrm{H}_{9} \mathrm{~N}$ & 9,845 & 9,048 & 4,270 & 14,114 & 13,317 & \\
\hline$s 683$ & 2,5-dimetilpiridina & $\mathrm{C}_{7} \mathrm{H}_{9} \mathrm{~N}$ & 10,062 & 9,088 & 4,270 & 14,332 & 13,358 & \\
\hline s682 & 2,4-dimetilpiridina & $\mathrm{C}_{7} \mathrm{H}_{9} \mathrm{~N}$ & 9,850 & 9,003 & 4,270 & 14,120 & 13,272 & \\
\hline s681 & 2,3-dimetilpiridina & $\mathrm{C}_{7} \mathrm{H}_{9} \mathrm{~N}$ & 9,883 & 8,975 & 4,270 & 14,153 & 13,244 & \\
\hline s688 & 3-etilpiridina & $\mathrm{C}_{7} \mathrm{H}_{9} \mathrm{~N}$ & 9,584 & 8,758 & 4,270 & 13,854 & 13,027 & \\
\hline s679 & 3-metilpiridina & $\mathrm{C}_{6} \mathrm{H}_{7} \mathrm{~N}$ & 8,513 & 7,732 & 3,548 & 12,060 & 11,280 & \\
\hline s678 & 2-metilpiridina & $\mathrm{C}_{6} \mathrm{H}_{7} \mathrm{~N}$ & 8,537 & 7,759 & 3.548 & 12,084 & 11,306 & \\
\hline s677 & Piridina & $\mathrm{C}_{5} \mathrm{H}_{5} \mathrm{~N}$ & 7,088 & 6,462 & 2,825 & 9,914 & 9,287 & 9,54 \\
\hline s675 & Dibenzofurano & $\mathrm{C}_{12} \mathrm{H}_{8} \mathrm{O}$ & 18,171 & 15,843 & 5,238 & 23,409 & 21,081 & \\
\hline s674 & Benzofurano & $\mathrm{C}_{8} \mathrm{H}_{6} \mathrm{O}$ & 11,248 & 9,996 & 3.722 & 14,970 & 13,718 & \\
\hline s702 & 3-cianopiridina & $\mathrm{C}_{6} \mathrm{H}_{4} \mathrm{~N}_{2}$ & 9,860 & 8,871 & 3,220 & 13,079 & 12,091 & \\
\hline s680 & 4-metilpiridina & $\mathrm{C}_{6} \mathrm{H}_{7} \mathrm{~N}$ & 8,456 & 7,702 & 3,548 & 12,004 & 11,249 & \\
\hline s694 & 2-cloropiridina & $\mathrm{C}_{5} \mathrm{H}_{4} \mathrm{NCl}$ & 8,981 & 8,041 & 3,920 & 12,902 & 11,961 & \\
\hline s700 & 4-metoxipiridina & $\mathrm{C}_{6} \mathrm{H}_{7} \mathrm{NO}$ & 9,392 & 8,528 & 3,780 & 13,172 & 12,308 & \\
\hline s699 & 2-metoxipiridina & $\mathrm{C}_{6} \mathrm{H}_{7} \mathrm{NO}$ & 9,529 & 8,606 & 3,780 & 13,309 & 12,386 & \\
\hline
\end{tabular}


Tabela 5.4 (continuação)

\begin{tabular}{|c|c|c|c|c|c|c|c|c|}
\hline No & Soluto & Fórmula & $\alpha_{D}\left(A^{3}\right)$ & $\alpha_{0}\left(\AA^{3}\right)$ & $\sum \alpha_{i}\left(\AA^{3}\right)$ & $\alpha_{\text {calc (D) }}\left(A^{3}\right)$ & $\alpha_{\text {calc (o) }}\left(\AA^{3}\right)$ & $\alpha_{\exp }\left(A^{3}\right)$ \\
\hline s698 & 4-bromopiridina & $\mathrm{C}_{5} \mathrm{H}_{4} \mathrm{NBr}$ & 8,999 & 8,243 & 4,855 & 13,854 & 13,098 & \\
\hline s697 & 3-bromopiridina & $\mathrm{C}_{5} \mathrm{H}_{4} \mathrm{NBr}$ & 9,149 & 8,221 & 4,855 & 14,004 & 13,076 & \\
\hline s687 & 2-etilpiridina & $\mathrm{C}_{7} \mathrm{H}_{9} \mathrm{~N}$ & 9,615 & 8,790 & 4,270 & 13,885 & 13,060 & \\
\hline s695 & 3-cloropiridina & $\mathrm{C}_{5} \mathrm{H}_{4} \mathrm{NCl}$ & 8,608 & 7,904 & 3,920 & 12,528 & 11,824 & \\
\hline s693 & 2,6-difluorpiridina & $\mathrm{C}_{5} \mathrm{H}_{3} \mathrm{NF}_{2}$ & 7,582 & 6,848 & 2,764 & 10,346 & 9,612 & \\
\hline$s 692$ & 3-fluorpiridina & $\mathrm{C}_{5} \mathrm{H}_{4} \mathrm{NF}$ & 7,383 & 6,688 & 2,795 & 10,178 & 9,483 & \\
\hline s691 & 2-fluorpiridina & $\mathrm{C}_{5} \mathrm{H}_{4} \mathrm{NF}$ & 7,329 & 6,651 & 2,795 & 10,124 & 9,446 & \\
\hline s690 & 4-n-propilpiridina & $\mathrm{C}_{8} \mathrm{H}_{11} \mathrm{~N}$ & 10,695 & 9,816 & 4,992 & 15,687 & 14,807 & \\
\hline s689 & 4-etilpiridina & $\mathrm{C}_{7} \mathrm{H}_{9} \mathrm{~N}$ & 9,526 & 8,722 & 4,270 & 13,795 & 12,991 & \\
\hline s696 & 4-cloropiridina & $\mathrm{C}_{5} \mathrm{H}_{4} \mathrm{NCl}$ & 8,785 & 7,898 & 3,920 & 12,705 & 11,819 & \\
\hline s673 & 2-metilfurano & $\mathrm{C}_{5} \mathrm{H}_{6} \mathrm{O}$ & 6,693 & 6,072 & 2,928 & 9,621 & 9,000 & \\
\hline s701 & 2-cianopiridina & $\mathrm{C}_{6} \mathrm{H}_{4} \mathrm{~N}_{2}$ & 9,832 & 8,858 & 3,220 & 13,052 & 12,078 & \\
\hline s714 & $n$-acetilpirrolidina & $\mathrm{C}_{6} \mathrm{H}_{11} \mathrm{NO}$ & 8,371 & 7,793 & 4,695 & 13,066 & 12,488 & \\
\hline s713 & $n$-metilpirrol & $\mathrm{C}_{5} \mathrm{H}_{7} \mathrm{~N}$ & 7,283 & 6,585 & 3,283 & 10,566 & 9,868 & \\
\hline s712 & Pirrol & $\mathrm{C}_{4} \mathrm{H}_{5} \mathrm{~N}$ & 5,855 & 5,299 & 2,561 & 8,415 & 7,860 & 9,20 \\
\hline s711 & $n$-etilpiperidina & $\mathrm{C}_{7} \mathrm{H}_{15} \mathrm{~N}$ & 8,826 & 8,316 & 5,642 & 14,467 & 13,958 & \\
\hline s710 & $n$-metilpiperidina & $\mathrm{C}_{6} \mathrm{H}_{13} \mathrm{~N}$ & 7,672 & 7,228 & 4,920 & 12,592 & 12,148 & \\
\hline s708 & Quinolina & $\mathrm{C}_{9} \mathrm{H}_{7} \mathrm{~N}$ & 13,727 & 12,111 & 4,342 & 18,069 & 16,453 & \\
\hline s707 & 4-acetilpiridina & $\mathrm{C}_{7} \mathrm{H}_{7} \mathrm{NO}$ & 10,214 & 9,305 & 4,045 & 14,259 & 13,349 & \\
\hline s706 & 3-acetilpiridina & $\mathrm{C}_{7} \mathrm{H}_{7} \mathrm{NO}$ & 10,285 & 9,355 & 4,045 & 14,330 & 13,400 & \\
\hline s705 & 4-formilpiridina & $\mathrm{C}_{6} \mathrm{H}_{5} \mathrm{NO}$ & 9,156 & 8,281 & 3,323 & 12,478 & 11,603 & \\
\hline s704 & 3-formilpiridina & $\mathrm{C}_{6} \mathrm{H}_{5} \mathrm{NO}$ & 9,220 & 8,327 & 3,323 & 12,542 & 11,650 & \\
\hline s703 & 4-cianopiridina & $\mathrm{C}_{6} \mathrm{H}_{4} \mathrm{~N} 2$ & 9,758 & 8,805 & 3,220 & 12,978 & 12,025 & \\
\hline \multicolumn{9}{|c|}{ Ácidos Carboxílicos } \\
\hline s204 & $\gamma$-butirolactona & $\mathrm{C}_{4} \mathrm{H}_{6} \mathrm{O}_{2}$ & 5,337 & 5,042 & 2,896 & 8,232 & 7,938 & \\
\hline s321 & Ácido dicloroacético & $\mathrm{C}_{2} \mathrm{H}_{2} \mathrm{O}_{2} \mathrm{Cl}_{2}$ & 5,951 & 5,463 & 4,099 & 10,050 & 9,562 & \\
\hline s301 & Ácido acético & $\mathrm{C}_{2} \mathrm{H}_{4} \mathrm{O}_{2}$ & 3,211 & 3,040 & 1,909 & 5,120 & 4,949 & 5,13 \\
\hline s302 & Ácido propanóico & $\mathrm{C}_{3} \mathrm{H}_{6} \mathrm{O}_{2}$ & 4,317 & 4,081 & 2,631 & 6,948 & 6,712 & \\
\hline s303 & Ácido butanóico & $\mathrm{C}_{4} \mathrm{H}_{8} \mathrm{O}_{2}$ & 5,434 & 5,135 & 3,353 & 8,787 & 8,488 & \\
\hline s304 & Ácido pentanóico & $\mathrm{C}_{5} \mathrm{H}_{10} \mathrm{O}_{2}$ & 6,584 & 6,219 & 4,075 & 10,659 & 10,295 & \\
\hline s300 & Ácido fórmico & $\mathrm{CH}_{2} \mathrm{O}_{2}$ & 2,068 & 1,960 & 1,187 & 3,255 & 3,147 & \\
\hline s305 & Ácido-3-metilbutanóico & $\mathrm{C}_{5} \mathrm{H}_{10} \mathrm{O}_{2}$ & 6,474 & 6,124 & 4,075 & 10,550 & 10,199 & \\
\hline s317 & Ácido hexadecanóico & $\mathrm{C}_{16} \mathrm{H}_{32} \mathrm{O}_{2}$ & 18,816 & 17,760 & 12,018 & 30,835 & 29,779 & \\
\hline s314 & Ácido-2-propilpentanóico & $\mathrm{C}_{8} \mathrm{H}_{16} \mathrm{O}_{2}$ & 9,768 & 9,233 & 6,242 & 16,010 & 15,475 & \\
\hline s315 & Ácido decanóico & $\mathrm{C}_{10} \mathrm{H}_{20} \mathrm{O}_{2}$ & 12,092 & 11,418 & 7,686 & 19,778 & 19,104 & \\
\hline s316 & Ácido tetradecanóico & $\mathrm{C}_{14} \mathrm{H}_{28} \mathrm{O}_{2}$ & 16,537 & 15,613 & 10,574 & 27,111 & 26,187 & \\
\hline s313 & Ácido-2-etilhexanóico & $\mathrm{C}_{8} \mathrm{H}_{16} \mathrm{O}_{2}$ & 9,879 & 9,330 & 6,242 & 16,121 & 15,572 & \\
\hline s312 & Ácido decanóico & $\mathrm{C}_{10} \mathrm{H}_{20} \mathrm{O}_{2}$ & 12,092 & 11,418 & 7,686 & 19,778 & 19,104 & \\
\hline s318 & Ácido octadecanóico & $\mathrm{C}_{18} \mathrm{H}_{36} \mathrm{O}_{2}$ & 20,984 & 19,809 & 13,463 & 34,446 & 33,271 & \\
\hline s319 & Ácido eicosanóico & $\mathrm{C}_{20} \mathrm{H}_{40} \mathrm{O}_{2}$ & 23,210 & 21,909 & 14,907 & 38,117 & 36,816 & \\
\hline s322 & Ácido tricloroacético & $\mathrm{C}_{2} \mathrm{HO}_{2} \mathrm{Cl}_{3}$ & 7,596 & 6,848 & 5,194 & 12,789 & 12,041 & \\
\hline s320 & Ácido cloroacético & $\mathrm{C}_{2} \mathrm{H}^{3} \mathrm{O}_{2} \mathrm{Cl}$ & 4,420 & 4,134 & 3,004 & 7,424 & 7,138 & \\
\hline s306 & Ácido hexanóico & $\mathrm{C}_{6} \mathrm{H}_{12} \mathrm{O}_{2}$ & 7,652 & 7,228 & 4,797 & 12,449 & 12,026 & \\
\hline s307 & Ácido-2-metilpentanóico & $\mathrm{C}_{6} \mathrm{H}_{12} \mathrm{O}_{2}$ & 7,667 & 7,243 & 4,797 & 12,465 & 12,041 & \\
\hline s308 & Ácido hexanóico & $\mathrm{C}_{7} \mathrm{H}_{14} \mathrm{O}_{2}$ & 8,761 & 8,276 & 5,520 & 14,281 & 13,795 & \\
\hline s310 & Ácido heptanóico & $\mathrm{C}_{7} \mathrm{H}_{14} \mathrm{O}_{2}$ & 8,809 & 8,318 & 5,520 & 14,328 & 13,838 & \\
\hline s311 & Ácido octanóico & $\mathrm{C}_{8} \mathrm{H}_{16} \mathrm{O}_{2}$ & 9,872 & 9,323 & 6,242 & 16,113 & 15,565 & \\
\hline s309 & Ácido-2-etilbutanóico & $\mathrm{C}_{6} \mathrm{H}_{12} \mathrm{O}_{2}$ & 7,573 & 7,159 & 4,797 & 12,370 & 11,957 & \\
\hline s584 & Ácido-4-etilbenzóico & $\mathrm{C}_{9} \mathrm{H}_{10} \mathrm{O}_{2}$ & 12,814 & 11,588 & 5,134 & 17,948 & 16,722 & \\
\hline
\end{tabular}


Tabela 5.4 (continuação)

\begin{tabular}{|c|c|c|c|c|c|c|c|c|}
\hline No & Soluto & Fórmula & $\alpha_{D}\left(\AA^{3}\right)$ & $\alpha_{0}\left(\AA^{3}\right)$ & $\overline{\Sigma \alpha_{i}\left(\AA^{3}\right)}$ & $\alpha_{\text {calc }(D)}\left(A^{3}\right)$ & $\alpha_{\text {calc (o) }}\left(\AA^{3}\right)$ & $\alpha_{\exp }\left(\AA^{3}\right)$ \\
\hline$\overline{\mathrm{s521}}$ & Acetato de fenila & $\mathrm{C}_{8} \mathrm{H}_{8} \mathrm{O}_{2}$ & 11,734 & 10,643 & 4,412 & 16,146 & 15,055 & \\
\hline s520 & 2-metil benzoato de metila & $\mathrm{C}_{9} \mathrm{H}_{10} \mathrm{O}_{2}$ & 12,813 & 11,654 & 5,134 & 17,947 & 16,788 & \\
\hline $\mathrm{s} 526$ & Acetato de 4-etilfenil & $\mathrm{C}_{10} \mathrm{H}_{12} \mathrm{O}_{2}$ & 14,050 & 12,789 & 5,856 & 19,906 & 18,646 & \\
\hline s527 & Acetato de benzila & $\mathrm{C}_{9} \mathrm{H}_{10} \mathrm{O}_{2}$ & 12,643 & 11,568 & 5,134 & 17,778 & 16,702 & \\
\hline s223 & Propianoato de etila & $\mathrm{C}_{5} \mathrm{H}_{10} \mathrm{O}_{2}$ & 6,848 & 6,454 & 4,075 & 10,923 & 10,530 & \\
\hline s224 & Propianoato de $n$-propila & $\mathrm{C}_{6} \mathrm{H}_{12} \mathrm{O}_{2}$ & 7,977 & 7,518 & 4,797 & 12,774 & 12,315 & \\
\hline s225 & Propianoato de isopropila & $\mathrm{C}_{6} \mathrm{H}_{12} \mathrm{O}_{2}$ & 7,925 & 7,476 & 4,797 & 12,722 & 12,273 & \\
\hline s221 & Acetato de alila & $\mathrm{C}_{5} \mathrm{H}_{8} \mathrm{O}_{2}$ & 7,169 & 6,668 & 3,618 & 10,787 & 10,286 & \\
\hline s211 & Acetato de metila & $\mathrm{C}_{3} \mathrm{H}_{6} \mathrm{O}_{2}$ & 4,505 & 4,253 & 2,631 & 7,137 & 6,884 & \\
\hline $\mathrm{s} 212^{-}$ & Acetato de etila & $\mathrm{C}_{4} \mathrm{H}_{8} \mathrm{O}_{2}$ & 5,724 & 5,399 & 3,353 & 9,077 & 8,752 & \\
\hline s213 & Acetato de $n$-propila & $\mathrm{C}_{5} \mathrm{H}_{10} \mathrm{O}_{2}$ & 6,847 & 6,457 & 4,075 & 10,922 & 10,533 & \\
\hline $\mathrm{s} 525$ & Acetato de 2-etilfenil & $\mathrm{C}_{10} \mathrm{H}_{12} \mathrm{O}_{2}$ & 14,065 & 12,790 & 5,856 & 19,921 & 18,646 & \\
\hline s214 & Acetato de isopropila & $\mathrm{C}_{5} \mathrm{H}_{10} \mathrm{O}_{2}$ & 6,664 & 6,355 & 4,075 & 10,739 & 10,430 & \\
\hline s215 & Acetato de $n$-butila & $\mathrm{C}_{6} \mathrm{H}_{12} \mathrm{O}_{2}$ & 7,978 & 7,523 & 4,797 & 12,775 & 12,320 & \\
\hline s531 & Dietil ftalato & $\mathrm{C}_{12} \mathrm{H}_{14} \mathrm{O}_{4}$ & 17,494 & 16,076 & 7,308 & 24,801 & 23,384 & \\
\hline s216 & Acetato de isobutila & $\mathrm{C}_{6} \mathrm{H}_{12} \mathrm{O}_{2}$ & 7,907 & 7,460 & 4,797 & 12,704 & 12,258 & \\
\hline s530 & Dimetil ftalato & $\mathrm{C}_{10} \mathrm{H}_{10} \mathrm{O}_{4}$ & 14,790 & 13,569 & 5,864 & 20,654 & 19,433 & \\
\hline s217 & Acetato de $n$-pentila & $\mathrm{C}_{7} \mathrm{H}_{14} \mathrm{O}_{2}$ & 9,092 & 8,574 & 5,520 & 14,611 & 14,094 & \\
\hline $\mathrm{s} 529$ & Fenil acetato de etila & $\mathrm{C}_{10} \mathrm{H}_{12} \mathrm{O}_{2}$ & 13,390 & 12,301 & 5,856 & 19,246 & 18,157 & \\
\hline s528 & Fenil acetato de metila & $\mathrm{C}_{9} \mathrm{H}_{10} \mathrm{O}_{2}$ & 12,164 & 11,151 & 5,134 & 17,299 & 16,285 & \\
\hline s218 & Acetato de isopentila & $\mathrm{C}_{7} \mathrm{H}_{14} \mathrm{O}_{2}$ & 9,064 & 8,552 & 5,520 & 14,583 & 14,071 & \\
\hline \multicolumn{9}{|c|}{ Éteres } \\
\hline s492 & $n$-propil fenil eter & $\mathrm{C}_{9} \mathrm{H}_{12} \mathrm{O}$ & 12,273 & 11,156 & 5,359 & 17,632 & 16,516 & \\
\hline s491 & Etil fenil eter & $\mathrm{C}_{8} \mathrm{H}_{10} \mathrm{O}$ & 11,100 & 10,061 & 4,637 & 15,737 & 14,698 & \\
\hline s490 & Metil fenil eter & $\mathrm{C}_{7} \mathrm{H}_{8} \mathrm{O}$ & 9,763 & 8,828 & 3,915 & 13,678 & 12,743 & \\
\hline s154 & Eter dietílico & $\mathrm{C}_{4} \mathrm{H}_{10} \mathrm{O}$ & 5,265 & 4,976 & 3,578 & 8,843 & 8,555 & \\
\hline s155 & Eter di- $n$-propílico & $\mathrm{C}_{6} \mathrm{H}_{14} \mathrm{O}$ & 7,498 & 7,083 & 5,022 & 12,520 & 12,106 & \\
\hline s156 & Eter diisopropílico & $\mathrm{C}_{6} \mathrm{H}_{14} \mathrm{O}$ & 7,397 & 7,003 & 5,022 & 12,420 & 12,026 & \\
\hline s157 & Eter di- $n$-butílico & $\mathrm{C}_{8} \mathrm{H}_{18} \mathrm{O}$ & 9,738 & 9,197 & 6,467 & 16,205 & 15,664 & \\
\hline s158 & Metil $n$-propíl eter & $\mathrm{C}_{4} \mathrm{H}_{10} \mathrm{O}$ & 5,207 & 4,921 & 3,578 & 8,785 & 8,499 & \\
\hline s159 & Etil $n$-butil eter & $\mathrm{C}_{6} \mathrm{H}_{14} \mathrm{O}$ & 7,494 & 7,080 & 5,022 & 12,517 & 12,103 & \\
\hline s153 & Eter dimetílico & $\mathrm{C}_{2} \mathrm{H}_{6} \mathrm{O}$ & 2,917 & 2,761 & 2,134 & 5,051 & 4,895 & 5,08 \\
\hline s168 & Tetrahidropirano & $\mathrm{C}_{5} \mathrm{H}_{10} \mathrm{O}$ & 6,047 & 5,717 & 3,843 & 9,890 & 9,560 & \\
\hline s169 & 1,4-dioxano & $\mathrm{C}_{4} \mathrm{H}_{8} \mathrm{O}_{2}$ & 5,500 & 5,209 & 3.353 & 8,853 & 8,562 & \\
\hline s165 & Tetrahidrofurano & $\mathrm{C}_{4} \mathrm{H}_{8} \mathrm{O}$ & 4,762 & 4,507 & 3,121 & 7,882 & 7,628 & \\
\hline s160 & Óxido de1,2-propileno & $\mathrm{C}_{3} \mathrm{H}_{6} \mathrm{O}$ & 3,857 & 3,650 & 2,399 & 6,256 & 6,049 & \\
\hline s167 & 2,5-dimetiltetrahidrofurano & $\mathrm{C}_{6} \mathrm{H}_{12} \mathrm{O}$ & 6,990 & 6,621 & 4,565 & 11,555 & 11,186 & \\
\hline s166 & 2-metiltetrahidrofurano & $\mathrm{C}_{5} \mathrm{H}_{10} \mathrm{O}$ & 5,865 & 5,555 & 3,843 & 9,708 & 9,398 & \\
\hline \multicolumn{9}{|c|}{ Gases } \\
\hline s014 & Dióxido de carbono & $\mathrm{CO}_{2}$ & 1,762 & 1,693 & 0,730 & 2,492 & 2,422 & \\
\hline s013 & Monóxido de carbono & $\mathrm{CO}$ & 1,239 & 1,197 & 0,497 & 1,736 & 1,694 & 1,96 \\
\hline s011 & Nitrogênio & $\mathrm{N}_{2}$ & 1,189 & 1,142 & 0,717 & 1,906 & 1,859 & 1,76 \\
\hline s010 & Oxgênio & $\mathrm{O}_{2}$ & 0,738 & 0,709 & 0,465 & 1,202 & 1,174 & 1,60 \\
\hline s009 & Hidrogênio & $\mathrm{H}_{2}$ & 0,211 & 0,202 & 0,457 & 0,668 & 0,659 & 0,79 \\
\hline s012 & Óxido nitroso & $\mathrm{N}_{2} \mathrm{O}$ & 2,028 & 1,918 & 0,949 & 2,977 & 2,868 & \\
\hline \multicolumn{9}{|c|}{ Compostos Halogenados } \\
\hline s099 & 1,2-dicloroetano & $\mathrm{C}_{2} \mathrm{H}_{4} \mathrm{C}_{12}$ & 4,887 & 4,500 & 4,091 & 8,978 & 8,592 & \\
\hline s139 & 1-bromooctano & $\mathrm{C}_{8} \mathrm{H}_{17} \mathrm{Br}$ & 10,967 & 10,180 & 8,264 & 19,231 & 18,444 & \\
\hline s140 & 1-bromodecano & $\mathrm{C}_{10} \mathrm{H}_{21} \mathrm{Br}$ & 13,183 & 12,274 & 9,708 & 22,891 & 21,982 & \\
\hline
\end{tabular}


Tabela 5.4 (continuação)

\begin{tabular}{|c|c|c|c|c|c|c|c|c|}
\hline No & Soluto & Fórmula & $\alpha_{D}\left(\AA^{3}\right)$ & $\alpha_{0}\left(\AA^{3}\right)$ & $\overline{\Sigma \alpha_{i}\left(\AA^{3}\right)}$ & $\alpha_{\text {calc (D) }}\left(\AA^{3}\right)$ & $\alpha_{\text {calc (o) }}\left(\AA^{3}\right)$ & $\alpha_{\exp }\left(\AA^{3}\right)$ \\
\hline s138 & 1-bromoheptano & $\mathrm{C}_{7} \mathrm{H}_{15} \mathrm{Br}$ & 9,845 & 9,122 & 7,542 & 17,386 & 16,664 & \\
\hline s141 & Bromociclohexano & $\mathrm{C}_{6} \mathrm{H}_{11} \mathrm{Br}$ & 8,760 & 8,043 & 6,362 & 15,123 & 14,405 & \\
\hline s142 & Iodometano & $\mathrm{CH}_{3} \mathrm{I}$ & 3,308 & 2,956 & 5,044 & 8,352 & 8,000 & \\
\hline s143 & Diiodometano & $\mathrm{CH}_{2} \mathrm{I}_{2}$ & 9,277 & 5,704 & 8,908 & 18,185 & 14,612 & 12,91 \\
\hline s144 & Iodoetano & $\mathrm{C}_{2} \mathrm{H}_{5} \mathrm{I}$ & 4,978 & 4,388 & 5,766 & 10,744 & 10,154 & \\
\hline s130 & 1,2-dibromoetano & $\mathrm{C}_{2} \mathrm{H}_{4} \mathrm{Br}_{2}$ & 6,820 & 5,954 & 5,961 & 12,781 & 11,915 & \\
\hline s131 & 1-bromopropano & $\mathrm{C}_{3} \mathrm{H}_{7} \mathrm{Br}$ & 5,333 & 4,878 & 4,653 & 9,987 & 9,532 & \\
\hline s132 & 2-bromopropano & $\mathrm{C}_{3} \mathrm{H}_{7} \mathrm{Br}$ & 5,582 & 5,034 & 4,653 & 10,235 & 9,687 & \\
\hline s133 - & 1-bromobutano & $\mathrm{C}_{4} \mathrm{H}_{9} \mathrm{Br}$ & 6,480 & 5,953 & 5,375 & 11,855 & 11,328 & \\
\hline s145 & 1-iodopropano & $\mathrm{C}_{3} \mathrm{H}_{7} \mathrm{I}$ & 6,053 & 5,427 & 6,488 & 12,541 & 11,915 & \\
\hline s134 & 1-bromo-2-metilpropano & $\mathrm{C}_{4} \mathrm{H}_{9} \mathrm{Br}$ & 6,331 & 5,832 & 5,375 & 11,706 & 11,207 & \\
\hline s135 & 2-bromo-2-metilpropano & $\mathrm{C}_{4} \mathrm{H}_{9} \mathrm{Br}$ & 6,756 & 6,103 & 5,375 & 12,132 & 11,478 & \\
\hline s136 & 1-bromopentano & $\mathrm{C}_{5} \mathrm{H}_{11} \mathrm{Br}$ & 7,608 & 7,014 & 6,098 & 13,705 & 13,112 & \\
\hline s137 & 1-bromohexano & $\mathrm{C}_{6} \mathrm{H}_{13} \mathrm{Br}$ & 8,726 & 8,068 & 6,820 & 15,545 & 14,887 & \\
\hline s146 & 1-iodobutano & $\mathrm{C}_{4} \mathrm{H}_{9} \mathrm{I}$ & 7,223 & 6,517 & 7,210 & 14,433 & 13,727 & \\
\hline s147 & 1-iodopentano & $\mathrm{C}_{5} \mathrm{H}_{11} \mathrm{I}$ & 8,366 & 7,590 & 7,932 & 16,298 & 15,522 & \\
\hline s148 & 1-iodohexano & $\mathrm{C}_{6} \mathrm{H}_{13} \mathrm{I}$ & 9,491 & 8,649 & 8,654 & 18,146 & 17,303 & \\
\hline s150 & Bromodiclorometano & $\mathrm{CHC}_{12} \mathrm{Br}$ & 6,698 & 5,460 & 5,399 & 12,097 & 10,859 & \\
\hline s151 & Polialotano & $\mathrm{C}_{2} \mathrm{HF}_{3} \mathrm{ClBr}$ & 5,936 & 5,127 & 4,935 & 10,870 & 10,062 & \\
\hline s152 & Teflurano & $\mathrm{C}_{2} \mathrm{HF}_{4} \mathrm{Br}$ & 4,562 & 4,027 & 3,809 & 8,371 & 7,836 & \\
\hline s149 & 1-iodoheptano & $\mathrm{C}_{7} \mathrm{H}_{15} \mathrm{I}$ & 10,616 & 9,705 & 9,376 & 19,992 & 19,082 & \\
\hline s111 & 1-cloro-2-metilpropano & $\mathrm{C}_{4} \mathrm{H}_{9} \mathrm{Cl}$ & 5,660 & 5,307 & 4,441 & 10,101 & 9,747 & \\
\hline s109 & 1,3-dicloropropano & $\mathrm{C}_{3} \mathrm{H}_{6} \mathrm{Cl}_{2}$ & 5,910 & 5,481 & 4,814 & 10,723 & 10,294 & \\
\hline s112 & 2-clorobutano & $\mathrm{C}_{4} \mathrm{H}_{9} \mathrm{Cl}$ & 5,802 & 5,418 & 4,441 & 10,242 & 9,859 & \\
\hline s113 & 2-cloro-2-metilpropano & $\mathrm{C}_{4} \mathrm{H}_{9} \mathrm{Cl}$ & 5,850 & 5,455 & 4,441 & 10,291 & 9,895 & \\
\hline s114 & 1,4-diclorobutano & $\mathrm{C}_{4} \mathrm{H}_{8} \mathrm{Cl}_{2}$ & 7,059 & 6,561 & 5,536 & 12,595 & 12,097 & \\
\hline s110 & 1-clorobutano & $\mathrm{C}_{4} \mathrm{H}_{9} \mathrm{Cl}$ & 5,774 & 5,402 & 4,441 & 10,214 & 9,843 & \\
\hline s091 & 1-fluorbutano & $\mathrm{C}_{4} \mathrm{H}_{9} \mathrm{~F}$ & 4,584 & 4,333 & 3,315 & 7,899 & 7,648 & \\
\hline s100 & 1,1,1-tricloroetano & $\mathrm{C}_{2} \mathrm{H}_{3} \mathrm{Cl}_{3}$ & 6,624 & 5,946 & 5,186 & 11,810 & 11,132 & \\
\hline s098 & 1,1-dicloroetano & $\mathrm{C}_{2} \mathrm{H}_{4} \mathrm{Cl}_{2}$ & 4,990 & 4,558 & 4,091 & 9,081 & 8,649 & \\
\hline s097 & Cloroetano & $\mathrm{C}_{2} \mathrm{H}_{5} \mathrm{Cl}$ & 3,544 & 3,296 & 2,997 & 6,540 & 6,292 & 6,41 \\
\hline s101 & 1,1,2-tricloroetano & $\mathrm{C}_{2} \mathrm{H}_{3} \mathrm{Cl}_{3}$ & 6,278 & 5,715 & 5,186 & 11,464 & 10,901 & \\
\hline s096 & Tetraclorometano & $\mathrm{CCl}_{4}$ & 7,268 & 6,338 & 5,559 & 12,827 & 11,897 & 10,48 \\
\hline s095 & Clorofórmio & $\mathrm{CHCl}_{3}$ & 5,363 & 4,770 & 4,464 & 9,827 & 9,234 & \\
\hline s102 & 1,1,2,2-tetracloroetano & $\mathrm{C}_{2} \mathrm{H}_{2} \mathrm{Cl}_{4}$ & 7,736 & 6,990 & 6,281 & 14,017 & 13,271 & \\
\hline s094 & Diclorometano & $\mathrm{CH}_{2} \mathrm{Cl}_{2}$ & 3,637 & 3,313 & 3,369 & 7,007 & 6,682 & 5,43 \\
\hline s093 & Clorometano & $\mathrm{CH}_{3} \mathrm{Cl}$ & 2,224 & 2,077 & 2,274 & 4,499 & 4,351 & 6,48 \\
\hline s108 & 1,2-dicloropropano & $\mathrm{C}_{3} \mathrm{H}_{6} \mathrm{Cl}_{2}$ & 6,056 & 5,591 & 4,814 & 10,869 & 10,404 & \\
\hline s092 & 1-fluorpentano & $\mathrm{C}_{5} \mathrm{H}_{11} \mathrm{~F}$ & 5,685 & 5,373 & 4,037 & 9,722 & 9,411 & \\
\hline s104 & Pentacloroetano & $\mathrm{C}_{2} \mathrm{HCl}_{5}$ & 9,229 & 8,257 & 7,376 & 16,606 & 15,633 & \\
\hline s090 & Tetrafluormetano & $\mathrm{CF}_{4}$ & 1,217 & 1,188 & 1,058 & 2,274 & 2,245 & \\
\hline s089 & Fluormetano & $\mathrm{CH}_{3} \mathrm{~F}$ & 1,237 & 1,173 & 1,149 & 2,386 & 2,322 & 2,59 \\
\hline s105 & Hexacloroetano & $\mathrm{C}_{2} \mathrm{Cl}_{6}$ & 10,802 & 9,594 & 8,471 & 19,273 & 18,065 & \\
\hline s106 & 1-cloropropano & $\mathrm{C}_{3} \mathrm{H}_{7} \mathrm{Cl}$ & 4,648 & 4,342 & 3,719 & 8,366 & 8,061 & \\
\hline s107 & 2-cloropropano & $\mathrm{C}_{3} \mathrm{H}_{7} \mathrm{Cl}$ & 4,747 & 4,417 & 3,719 & 8,466 & 8,136 & \\
\hline s103 & 1,1,1,2-tetracloroetano & $\mathrm{C}_{2} \mathrm{H}_{2} \mathrm{Cl}_{4}$ & 7,813 & 7,021 & 6,281 & 14,095 & 13,302 & \\
\hline s123 & Tricloroeteno & $\mathrm{C}_{2} \mathrm{HCl}_{3}$ & 7,246 & 6,230 & 4,729 & 11,975 & 10,959 & \\
\hline s124 & Tetracloroeteno & $\mathrm{C}_{2} \mathrm{Cl}_{4}$ & 9,367 & 7,821 & 5,824 & 15,191 & 13,645 & \\
\hline s125 & 1-cloro-2-propeno & $\mathrm{C}_{3} \mathrm{H}_{5} \mathrm{Cl}$ & 5,131 & 4,673 & 3,261 & 8,393 & 7,934 & \\
\hline
\end{tabular}


Tabela 5.4 (continuação)

\begin{tabular}{|c|c|c|c|c|c|c|c|c|}
\hline No & Soluto & Fórmula & $\alpha_{D}\left(\AA^{3}\right)$ & $\alpha_{0}\left(\AA^{3}\right)$ & $\Sigma \alpha_{\mathrm{i}}\left(\AA^{3}\right)$ & $\alpha_{\text {calc }(D)}\left(\AA^{3}\right)$ & $\alpha_{\text {calc (o) }}\left(\AA^{3}\right)$ & $\alpha_{\text {exp }}\left(\AA^{3}\right)$ \\
\hline$\overline{s 122}$ & trans-1,2-dicloroeteno & $\overline{\mathrm{C}_{2} \mathrm{H}_{2} \mathrm{Cl}_{2}}$ & 5,509 & 4,850 & 3,634 & 9,143 & 8,484 & \\
\hline s127 & Dibromometano & $\mathrm{CH}_{2} \mathrm{Br}_{2}$ & 4,898 & 4,142 & 5,239 & 10,136 & 9,380 & 8,69 \\
\hline s128 & Tribromometano & $\mathrm{CHBr}_{3}$ & 7,494 & 5,922 & 7,268 & 14,762 & 13,190 & 11,61 \\
\hline s129 & Bromoetano & $\mathrm{C}_{2} \mathrm{H}_{5} \mathrm{Br}$ & 4,260 & 3,849 & 3,931 & 8,191 & 7,781 & 7,50 \\
\hline s126 & Bromometano & $\mathrm{CH}_{3} \mathrm{Br}$ & 2,772 & 2,529 & 3,209 & 5,981 & 5,738 & \\
\hline s115 & 1-cloropentano & $\mathrm{C}_{5} \mathrm{H}_{11} \mathrm{Cl}$ & 6,894 & 6,459 & 5,163 & 12,057 & 11,621 & \\
\hline s116 & 1-clorohexano & $\mathrm{C}_{6} \mathrm{H}_{13} \mathrm{Cl}$ & 8,008 & 7,509 & 5,885 & 13,892 & 13,394 & \\
\hline s117 & 1-cloroheptano & $\mathrm{C}_{7} \mathrm{H}_{15} \mathrm{Cl}$ & 9,122 & 8,560 & 6,607 & 15,729 & 15,167 & \\
\hline s118 - & 1-clorooctano & $\mathrm{C}_{8} \mathrm{H}_{17} \mathrm{Cl}$ & 10,234 & 9,609 & 7,329 & 17,563 & 16,938 & \\
\hline s119 & Cloroeteno & $\mathrm{C}_{2} \mathrm{H}_{3} \mathrm{Cl}$ & 3,753 & 3,402 & 2,539 & 6,292 & 5,941 & . \\
\hline s120 & 1,1-dicloroeteno & $\mathrm{C}_{2} \mathrm{H}_{2} \mathrm{Cl}_{2}$ & 5,318 & 4,726 & 3,634 & 8,952 & 8,360 & \\
\hline s121 & cis-1,2-dicloroeteno & $\mathrm{C}_{2} \mathrm{H}_{2} \mathrm{Cl}_{2}$ & 5,282 & 4,658 & 3,634 & 8,916 & 8,292 & \\
\hline s164 & Fluoroxeno & $\mathrm{C}_{4} \mathrm{H}_{5} \mathrm{OF}_{3}$ & 5,573 & 5,215 & 3,029 & 8,602 & 8,244 & \\
\hline s163 & Enflurano & $\mathrm{C}_{3} \mathrm{H}_{2} \mathrm{OF}_{5} \mathrm{Cl}$ & 5,794 & 5,235 & 3,799 & 9,592 & 9,034 & \\
\hline s162 & Isoflurano & $\mathrm{C}_{3} \mathrm{H}_{2} \mathrm{OF}_{5} \mathrm{Cl}$ & 5,794 & 5,314 & 3,799 & 9,593 & 9,112 & \\
\hline s161 & Metoxiflurano & $\mathrm{C}_{3} \mathrm{H}_{4} \mathrm{OF}_{2} \mathrm{Cl}_{2}$ & 7,056 & 6,229 & 4,985 & 12,041 & 11,214 & \\
\hline \multicolumn{9}{|c|}{ Cetonas } \\
\hline s203 & 5-hexan-2-ona & $\mathrm{C}_{6} \mathrm{H}_{10} \mathrm{O}$ & 7,538 & 7,016 & 4,108 & 11,646 & 11,124 & \\
\hline s202 & Ciclododecanono & $\mathrm{C}_{12} \mathrm{H}_{22} \mathrm{O}$ & 13,782 & 13,000 & 8,440 & 22,222 & 21,441 & \\
\hline s195 & 5-nonanona & $\mathrm{C}_{9} \mathrm{H}_{18} \mathrm{O}$ & 10,534 & 9,921 & 6,731 & 17,265 & 16,652 & \\
\hline s196 & 5-metil-2-octanona & $\mathrm{C}_{9} \mathrm{H}_{18} \mathrm{O}$ & 10,527 & 9,924 & 6,731 & 17,258 & 16,655 & \\
\hline s197 & Di-butil cetona & $\mathrm{C}_{9} \mathrm{H}_{18} \mathrm{O}$ & 10,362 & 9,774 & 6,731 & 17,094 & 16,506 & \\
\hline s198 & 2-decanona & $\mathrm{C}_{10} \mathrm{H}_{20} \mathrm{O}$ & 11,642 & 10,973 & 7,453 & 19,096 & 18,427 & \\
\hline s199 & 2-undecanona & $\mathrm{C}_{11} \mathrm{H}_{22} \mathrm{O}$ & 12,755 & 12,022 & 8,176 & 20,930 & 20,198 & \\
\hline s 200 & Ciclopentanona & $\mathrm{C}_{5} \mathrm{H}_{8} \mathrm{O}$ & 5,817 & 5,491 & 3,386 & 9,203 & 8,877 & \\
\hline s201 & Ciclohexanona & $\mathrm{C}_{6} \mathrm{H}_{10} \mathrm{O}$ & 7,046 & 6,640 & 4,108 & 11,154 & 10,747 & \\
\hline s187 & 3-hexanona & $\mathrm{C}_{6} \mathrm{H}_{12} \mathrm{O}$ & 7,234 & 6,804 & 4,565 & 11,799 & 11,369 & \\
\hline s186 & 2-hexanona & $\mathrm{C}_{6} \mathrm{H}_{12} \mathrm{O}$ & 7,201 & 6,782 & 4,565 & 11,766 & 11,347 & \\
\hline s188 & 4-metil-2-pentanona & $\mathrm{C}_{6} \mathrm{H}_{12} \mathrm{O}$ & 7,099 & 6,696 & 4,565 & 11,664 & 11,261 & \\
\hline s185 & 3-metil-2-butanona & $\mathrm{C}_{5} \mathrm{H}_{10} \mathrm{O}$ & 6,021 & 5,674 & 3,843 & 9,864 & 9,517 & \\
\hline s189 & 3,3-dimetil-2-butanona & $\mathrm{C}_{6} \mathrm{H}_{12} \mathrm{O}$ & 7,069 & 6,672 & 4,565 & 11,634 & 11,237 & \\
\hline s181 & Propanona & $\mathrm{C}_{3} \mathrm{H}_{6} \mathrm{O}$ & 3,857 & 3,635 & 2,399 & 6,256 & 6,034 & \\
\hline s182 & Butanona & $\mathrm{C}_{4} \mathrm{H}_{8} \mathrm{O}$ & 4,957 & 4,670 & 3,121 & 8,078 & 7,791 & \\
\hline s184 & 3-pentanona & $\mathrm{C}_{5} \mathrm{H}_{10} \mathrm{O}$ & 6,090 & 5,730 & 3,843 & 9,933 & 9,573 & \\
\hline s183 & 2-pentanona & $\mathrm{C}_{5} \mathrm{H}_{10} \mathrm{O}$ & 6,091 & 5,735 & 3,843 & 9,934 & 9,578 & \\
\hline s191 & 4-heptanona & $\mathrm{C}_{7} \mathrm{H}_{14} \mathrm{O}$ & 8,314 & 7,828 & 5,287 & 13,602 & 13,115 & \\
\hline s192 & 5-metil-2-hexanona & $\mathrm{C}_{7} \mathrm{H}_{14} \mathrm{O}$ & 8,278 & 7,803 & 5,287 & 13,565 & 13,091 & \\
\hline s193 & 2-octanona & $\mathrm{C}_{8} \mathrm{H}_{16} \mathrm{O}$ & 9,409 & 8,866 & 6,009 & 15,418 & 14,875 & \\
\hline s194 & 2-nonanona & $\mathrm{C}_{9} \mathrm{H}_{18} \mathrm{O}$ & 10,531 & 9,924 & 6,731 & 17,262 & 16,656 & \\
\hline s190 & 2-heptanona & $\mathrm{C}_{7} \mathrm{H}_{14} \mathrm{O}$ & 8,307 & 7,826 & 5,287 & 13,594 & 13,113 & \\
\hline \multicolumn{9}{|c|}{ Compostos Multifuncionais } \\
\hline s550 & 4-aminoacetofenona & $\mathrm{C}_{8} \mathrm{H}_{9} \mathrm{NO}$ & 13,042 & 11,393 & 4,767 & 17,809 & 16,159 & \\
\hline \multicolumn{9}{|c|}{ Naftalenos } \\
\hline s430 & 1,7-dimetilnaftaleno & $\mathrm{C}_{12} \mathrm{H}_{12}$ & 17,269 & 15,114 & 5,921 & 23,190 & 21,035 & \\
\hline s558 & 2-naftilamina & $\mathrm{C}_{10} \mathrm{H}_{9} \mathrm{~N}$ & 16,749 & 14,280 & 5,064 & 21,813 & 19,343 & \\
\hline s557 & 1-naftilamina & $\mathrm{C}_{10} \mathrm{H}_{9} \mathrm{~N}$ & 16,497 & 14,007 & 5,064 & 21,561 & 19,071 & \\
\hline$s 423$ & Naftaleno & $\mathrm{C}_{10} \mathrm{H}_{8}$ & 14,134 & 12,423 & 4,477 & 18,611 & 16,899 & \\
\hline s429 & 1,5-dimetilnaftaleno & $\mathrm{C}_{12} \mathrm{H}_{12}$ & 17,087 & 14,962 & 5,921 & 23,008 & 20,883 & \\
\hline s428 & 1,4-dimetilnaftaleno & $\mathrm{C}_{12} \mathrm{H}_{12}$ & 17,169 & 14,990 & 5,921 & 23,089 & 20,911 & \\
\hline
\end{tabular}


Tabela 5.4 (continuação)

\begin{tabular}{|c|c|c|c|c|c|c|c|c|}
\hline No & Soluto & Fórmula & $\alpha_{D}\left(\AA^{3}\right)$ & $\alpha_{0}\left(A^{3}\right)$ & $\overline{\Sigma \alpha_{i}\left(\AA^{3}\right)}$ & $\bar{~} \alpha_{\text {calc (D) }}\left(\AA^{3}\right)$ & $\alpha_{\text {calc (o) }}\left(\AA^{3}\right)$ & $\overline{\alpha_{\exp }\left(\AA^{3}\right)}$ \\
\hline$\overline{s 427}$ & 1,3-dimetilnaftaleno & $\overline{\mathrm{C}_{12} \mathrm{H}_{12}}$ & 17,238 & 15,097 & 5,921 & 23,159 & 21,018 & \\
\hline$s 426$ & 1,2-dimetilnaftaleno & $\mathrm{C}_{12} \mathrm{H}_{12}$ & 17,132 & 15,027 & 5,921 & 23,053 & 20,947 & \\
\hline$s 425$ & 2-metilnaftaleno & $\mathrm{C}_{11} \mathrm{H}_{10}$ & 15,595 & 13,681 & 5,199 & 20,794 & 18,880 & \\
\hline s431 & 1,8-dimetilnaftaleno & $\mathrm{C}_{12} \mathrm{H}_{12}$ & 17,141 & 14,978 & 5,921 & 23,062 & 20,899 & \\
\hline$s 424$ & 1-metilnaftaleno & $\mathrm{C}_{11} \mathrm{H}_{10}$ & 15,638 & 13,705 & 5,199 & 20,836 & 18,904 & \\
\hline s432 & 2,3-dimetilnaftaleno & $\mathrm{C}_{12} \mathrm{H}_{12}$ & 17,336 & 15,204 & 5,921 & 23,257 & 21,125 & \\
\hline$s 439$ & Acenafteno & $\mathrm{C}_{12} \mathrm{H}_{10}$ & 16,617 & 14,584 & 5,463 & 22,080 & 20,048 & \\
\hline$s 436$ & 2,3,6-trimetilnaftaleno & $\mathrm{C}_{13} \mathrm{H}_{14}$ & 19,011 & 16,667 & 6,643 & 25,654 & 23,310 & \\
\hline s435 & 2-etilnaftaleno & $\mathrm{C}_{12} \mathrm{H}_{12}$ & 16,955 & 14,948 & 5,921 & 22,876 & 20,869 & \\
\hline$s 434$ & 1-etilnaftaleno & $\mathrm{C}_{12} \mathrm{H}_{12}$ & 16,714 & 14,752 & 5,921 & 22,634 & 20,673 & \\
\hline s433 & 2,6-dimetilnaftaleno & $\mathrm{C}_{12} \mathrm{H}_{12}$ & 17,115 & 14,970 & 5,921 & 23,036 & 20,890 & \\
\hline & & Anilinas & Compost & s Hetero & íclicos & & & \\
\hline s556 & $N, N$-dimetilanilina & $\mathrm{C}_{8} \mathrm{H}_{11} \mathrm{~N}$ & 12,073 & 10,717 & 4,992 & 17,065 & 15,709 & \\
\hline s555 & $N$-metilanilina & $\mathrm{C}_{7} \mathrm{H}_{9} \mathrm{~N}$ & 10,759 & 9,515 & 4,270 & 15,028 & 13,784 & \\
\hline s549 & 4-nitroanilina & $\mathrm{C}_{6} \mathrm{H}_{6} \mathrm{~N}_{2} \mathrm{O}_{2}$ & 12,908 & 10,963 & 4,142 & 17,050 & 15,105 & \\
\hline s548 & 3-nitroanilina & $\mathrm{C}_{6} \mathrm{H}_{6} \mathrm{~N}_{2} \mathrm{O}_{2}$ & 12,110 & 10,536 & 4,142 & 16,252 & 14,678 & \\
\hline s547 & 2-nitroanilina & $\mathrm{C}_{6} \mathrm{H}_{6} \mathrm{~N}_{2} \mathrm{O}_{2}$ & 12,649 & 10,741 & 4,142 & 16,791 & 14,883 & \\
\hline s546 & 4-metoxianilina & $\mathrm{C}_{7} \mathrm{H}_{9} \mathrm{NO}$ & 12,099 & 10,497 & 4,502 & 16,601 & 14,999 & \\
\hline s545 & 3-metoxianilina & $\mathrm{C}_{7} \mathrm{H}_{9} \mathrm{NO}$ & 11,756 & 10,362 & 4,502 & 16,258 & 14,864 & \\
\hline s544 & 2-metioxianilina & $\mathrm{C}_{7} \mathrm{H}_{9} \mathrm{NO}$ & 11,525 & 10,185 & 4,502 & 16,027 & 14,687 & \\
\hline s543 & 4-cloroanilina & $\mathrm{C}_{6} \mathrm{H}_{6} \mathrm{NCl}$ & 11,464 & 9,872 & 4,642 & 16,106 & 14,514 & \\
\hline s540 & 2,6-dimetilanilina & $\mathrm{C}_{8} \mathrm{H}_{11} \mathrm{~N}$ & 12,016 & 10,671 & 4,992 & 17,007 & 15,663 & \\
\hline s539 & $p$-toluidina & $\mathrm{C}_{7} \mathrm{H}_{9} \mathrm{~N}$ & 11,038 & 9,644 & 4,270 & 15,307 & 13,914 & \\
\hline s538 & $m$-toluidina & $\mathrm{C}_{7} \mathrm{H}_{9} \mathrm{~N}$ & 10,778 & 9,525 & 4,270 & 15,048 & 13,795 & \\
\hline s537 & $o$-toluidina & $\mathrm{C}_{7} \mathrm{H}_{9} \mathrm{~N}$ & 10,709 & 9,472 & 4,270 & 14,979 & 13,742 & \\
\hline s536 & Anilina & $\mathrm{C}_{6} \mathrm{H}_{7} \mathrm{~N}$ & 9,405 & 8,278 & 3,548 & 12,953 & 11,826 & 12,07 \\
\hline s542 & 3-cloroanilina & $\mathrm{C}_{6} \mathrm{H}_{6} \mathrm{NCl}$ & 11,199 & 9,772 & 4,642 & 15,841 & 14,415 & \\
\hline s541 & 2-cloroanilina & $\mathrm{C}_{6} \mathrm{H}_{6} \mathrm{NCl}$ & 11,019 & 9,624 & 4,642 & 15,662 & 14,267 & \\
\hline s738 & 2,1-benzisoxazol & $\mathrm{C}_{7} \mathrm{H}_{5} \mathrm{NO}$ & 12,187 & 10,263 & 3,587 & 15,775 & 13,850 & \\
\hline s737 & 1,2-benzisoxazol & $\mathrm{C}_{7} \mathrm{H}_{5} \mathrm{NO}$ & 10,824 & 9,690 & 3,587 & 14,411 & 13,278 & \\
\hline s736 & Isoxazol & $\mathrm{C}_{3} \mathrm{H}_{3} \mathrm{NO}$ & 4,944 & 4,545 & 2,071 & 7,015 & 6,616 & \\
\hline s735 & Benzoxazol & $\mathrm{C}_{7} \mathrm{H}_{5} \mathrm{NO}$ & 10,801 & 9,667 & 3,587 & 14,388 & 13,254 & \\
\hline s734 & Oxazol & $\mathrm{C}_{3} \mathrm{H}_{3} \mathrm{NO}$ & 4,857 & 4,469 & 2,071 & 6,928 & 6,540 & \\
\hline s716 & Indol & $\mathrm{C}_{8} \mathrm{H}_{7} \mathrm{~N}$ & 11,972 & 10,824 & 4,077 & 16,049 & 14,901 & \\
\hline s715 & Acridina & $\mathrm{C}_{13} \mathrm{H}_{9} \mathrm{~N}$ & 22,917 & 19,161 & 5,858 & 28,775 & 25,019 & \\
\hline s718 & Imidazol & $\mathrm{C}_{3} \mathrm{H}_{4} \mathrm{~N}_{2}$ & 5,513 & 5,020 & 2,426 & 7,939 & 7,446 & \\
\hline s709 & Piperidina & $\mathrm{C}_{5} \mathrm{H}_{11} \mathrm{~N}$ & 6,477 & 6,101 & 4,198 & 10,674 & 10,298 & \\
\hline s725 & Quinazolina & $\mathrm{C}_{8} \mathrm{H}_{6} \mathrm{~N}_{2}$ & 13,243 & 11,703 & 4,207 & 17,449 & 15,909 & \\
\hline s727 & Cinolina & $\mathrm{C}_{8} \mathrm{H}_{6} \mathrm{~N}_{2}$ & 13,382 & 11,736 & 4,207 & 17,589 & 15,943 & \\
\hline s717 & Pirazol & $\mathrm{C}_{3} \mathrm{H}_{4} \mathrm{~N}_{2}$ & 5,525 & 5,011 & 2,426 & 7,951 & 7,437 & \\
\hline s724 & Pirimidina & $\mathrm{C}_{4} \mathrm{H}_{4} \mathrm{~N}_{2}$ & 6,755 & 6,156 & 2,690 & 9,446 & 8,847 & \\
\hline s723 & Quinoxalina & $\mathrm{C}_{8} \mathrm{H}_{6} \mathrm{~N}_{2}$ & 13,445 & 11,852 & 4,207 & 17,651 & 16,058 & \\
\hline s722 & 2,5-dimetilpirazina & $\mathrm{C}_{6} \mathrm{H}_{8} \mathrm{~N}_{2}$ & 10,016 & 8,896 & 4,135 & 14,151 & 13,031 & \\
\hline s721 & 2-metilpirazina & $\mathrm{C}_{5} \mathrm{H}_{6} \mathrm{~N}_{2}$ & 8,331 & 7,525 & 3,413 & 11,744 & 10,938 & \\
\hline s720 & Pirazina & $\mathrm{C}_{4} \mathrm{H}_{4} \mathrm{~N}_{2}$ & 6,853 & 6,209 & 2,690 & 9,544 & 8,899 & \\
\hline s719 & $N$-metilimidazol & $\mathrm{C}_{4} \mathrm{H}_{6} \mathrm{~N}_{2}$ & 6,888 & 6,273 & 3,148 & 10,036 & 9,421 & \\
\hline s726 & Piridazina & $\mathrm{C}_{4} \mathrm{H}_{4} \mathrm{~N}_{2}$ & 6,847 & 6,190 & 2,690 & 9,537 & 8,880 & \\
\hline \multicolumn{9}{|c|}{ Nitrilas } \\
\hline s250 & 1-cianopropano & $\mathrm{C}_{4} \mathrm{H}_{7} \mathrm{~N}$ & 5,200 & 4,905 & 3,018 & 8,218 & 7,923 & \\
\hline
\end{tabular}


Tabela 5.4 (continuação)

\begin{tabular}{|c|c|c|c|c|c|c|c|c|}
\hline No & Soluto & Fórmula & $\alpha_{D}\left(A^{3}\right)$ & $\overline{\alpha_{1}\left(\AA^{3}\right)}$ & $\sum \alpha_{i}\left(\AA^{3}\right)$ & $\left(\alpha_{\text {calc }}(1)\right)\left(\AA^{\prime}\right)$ & $\alpha_{\text {calc (o) }}\left(\AA^{3}\right)$ & $\alpha_{\text {exp }}\left(A^{\prime}\right)$ \\
\hline $\mathrm{s} 251$ & 2-cianopropano & $\mathrm{C}_{4} \mathrm{H}_{7} \mathrm{~N}$ & 5,128 & 4,845 & 3,018 & 8,146 & 7,863 & \\
\hline $\mathrm{s} 252$ & I-cianobutano & $\mathrm{C}_{5} \mathrm{H}_{9} \mathrm{~N}$ & 6,317 & 5,960 & 3,740 & 10,057 & 9,700 & \\
\hline $\mathrm{s} 25.3$ & 1-ciano-2-metilpropano & $\mathrm{C}_{5} \mathrm{H}_{9} \mathrm{~N}$ & 6,247 & 5,899 & 3,740 & 9,987 & 9,639 & \\
\hline $\mathrm{s} 254$ & 2-cianobutano & $\mathrm{C}_{5} \mathrm{H}_{9} \mathrm{~N}$ & 6,235 & 5,889 & 3,740 & 9,975 & 9,629 & \\
\hline $\mathrm{s} 255$ & 2-ciano-2-metilpropano & $\mathrm{C}_{5} \mathrm{H}_{9} \mathrm{~N}$ & 6,164 & 5,832 & 3,740 & 9,904 & 9,572 & \\
\hline s256 & 1-cianopentano & $\mathrm{C}_{6} \mathrm{H}_{11} \mathrm{~N}$ & 7,434 & 7,013 & 4,462 & 11,896 & 11,476 & \\
\hline s248 & Acetonitrila & $\mathrm{C}_{2} \mathrm{H}_{3} \mathrm{~N}$ & 2,925 & 2,760 & 1,574 & 4,499 & 4,334 & \\
\hline s249 & Propionitrila & $\mathrm{C}_{3} \mathrm{H}_{5} \mathrm{~N}$ & 4,052 & 3,824 & 2,296 & 6,348 & 6,120 & \\
\hline s.533 & 2-metilbenzonitrila & $\mathrm{C}_{8} \mathrm{H}_{7} \mathrm{~N}$ & 11,349 & 10,383 & 4,077 & 15,426 & 14,460 & \\
\hline 5.532 & Benzonitrila & $\mathrm{C}_{7} \mathrm{H}_{5} \mathrm{~N}$ & 10,185 & 9,162 & 3,355 & 13,540 & 12,517 & \\
\hline s535 & Fenilacetonitrila & $\mathrm{C}_{8} \mathrm{H}_{7} \mathrm{~N}$ & 10,647 & 9,731 & 4,077 & 14,723 & 13,808 & \\
\hline \multicolumn{9}{|c|}{ Fenóis } \\
\hline s642 & 4-cianofenol & $\mathrm{C}_{7} \mathrm{H}_{5} \mathrm{NO}$ & 11,497 & 10,144 & 3,587 & 15,084 & 13,731 & \\
\hline$s 649$ & 4-hidroxibenzoato de metila & $\mathrm{C}_{8} \mathrm{H}_{8} \mathrm{O}_{3}$ & 12,716 & 11,376 & 4,644 & 17,360 & 16,021 & \\
\hline s652 & 2-naftol & $\mathrm{C}_{10} \mathrm{H}_{8} \mathrm{O}$ & 15,265 & 13,305 & 4,709 & 19,974 & 18,014 & \\
\hline s651 & 1-naftol & $\mathrm{C}_{10} \mathrm{H}_{8} \mathrm{O}$ & 15,277 & 13,240 & 4,709 & 19,986 & 17,949 & \\
\hline$s 648$ & Hidroquinona & $\mathrm{C}_{6} \mathrm{H}_{6} \mathrm{O}_{2}$ & 9,455 & 8,348 & 3,425 & 12,880 & 11,773 & \\
\hline s647 & Resorcinol & $\mathrm{C}_{6} \mathrm{H}_{6} \mathrm{O}_{2}$ & 9,267 & 8,271 & 3,425 & 12,692 & 11,696 & \\
\hline$s 646$ & Catecol & $\mathrm{C}_{6} \mathrm{H}_{6} \mathrm{O}_{2}$ & 9,225 & 8,233 & 3,425 & 12,651 & 11,658 & \\
\hline $\mathrm{s} 645$ & 4-nitrofenol & $\mathrm{C}_{6} \mathrm{H}_{5} \mathrm{NO}_{3}$ & 11,085 & 9,842 & 3,787 & 14,873 & 13,629 & \\
\hline$s 644$ & 3-nitrofenol & $\mathrm{C}_{6} \mathrm{H}_{5} \mathrm{NO}_{3}$ & 9,696 & 9,722 & 3,787 & 13,483 & 13,510 & \\
\hline $\mathrm{s} 643$ & 2-nitrofenol & $\mathrm{C}_{6} \mathrm{H}_{5} \mathrm{NO}_{3}$ & 11,051 & 9,768 & 3,787 & 14,839 & 13,556 & \\
\hline s650 & 4-hidroxibenzoato de $n$-butila & $\mathrm{C}_{11} \mathrm{H}_{14} \mathrm{O}_{3}$ & 16,275 & 14,732 & 6,811 & 23,086 & 21,543 & \\
\hline$s 620$ & 4-fenilfenol & $\mathrm{C}_{12} \mathrm{H}_{10} \mathrm{O}$ & 19,023 & 16,224 & 5,696 & 24,719 & 21,920 & \\
\hline$s 589$ & Fenol & $\mathrm{C}_{6} \mathrm{H}_{6} \mathrm{O}$ & 8,350 & 7,520 & 3,193 & 11,543 & 10,713 & \\
\hline$\$ 618$ & 2-fenilfenol & $\mathrm{C}_{12} \mathrm{H}_{10} \mathrm{O}$ & 18,454 & 15,961 & 5,696 & 24,150 & 21,657 & \\
\hline s608 & 3-propilfenol & $\mathrm{C}_{4} \mathrm{H}_{12} \mathrm{O}$ & 12,080 & 10,975 & 5,359 & 17,439 & 16,334 & \\
\hline s617 & 4-tert-pentilfenol & $\mathrm{C}_{11} \mathrm{H}_{10} \mathrm{O}$ & 14,474 & 13,155 & 6,803 & 21,277 & 19,959 & \\
\hline s616 & 2-isopropil-5-metilfenol & $\mathrm{C}_{10} \mathrm{H}_{14} \mathrm{O}$ & 13,406 & 12,144 & 6,081 & 19,487 & 18,225 & \\
\hline 5628 & 3,5-diclorofenol & $\mathrm{C}_{6} \mathrm{H}_{4} \mathrm{OCl}_{2}$ & 11,974 & 10,556 & 5,383 & 17,356 & 15,939 & \\
\hline s619 & 3-fenilfenol & $\mathrm{C}_{12} \mathrm{H}_{10} \mathrm{O}$ & 18,301 & 15,910 & 5,696 & 23,997 & 21,606 & \\
\hline s627 & 3.4-diclorofenol & $\mathrm{C}_{6} \mathrm{H}_{4} \mathrm{OCl}_{2}$ & 11,911 & 10,443 & 5,383 & 17,294 & 15,825 & \\
\hline s626 & 4-clorofenol & $\mathrm{C}_{6} \mathrm{H}_{5} \mathrm{OCl}$ & 10,271 & 9,003 & 4,288 & 14,559 & 13,291 & \\
\hline$\$ 625$ & 3-clorofenol & $\mathrm{C}_{6}, \mathrm{H}_{5} \mathrm{OCl}$ & 10,099 & 8,995 & 4,288 & 14,387 & 13,282 & \\
\hline$s 621$ & 2-fluorfenol & $\mathrm{C}_{6} \mathrm{H}_{5} \mathrm{OF}$ & 8,664 & 7,754 & 3,162 & 11,826 & 10,916 & \\
\hline$s 624$ & 2-clorofenol & $\mathrm{C}_{6} \mathrm{H}_{5} \mathrm{OCl}$ & 10,055 & 8,934 & 4,288 & 14,342 & 13,222 & \\
\hline s623 & 4-fluorfenol & $\mathrm{C}_{6} \mathrm{H}_{5} \mathrm{OF}$ & 8,730 & 7,779 & 3,162 & 11,892 & 10,941 & \\
\hline s622 & 3-fluorfenol & $\mathrm{C}_{6} \mathrm{H}_{5} \mathrm{OF}$ & 8,594 & 7,717 & 3,162 & 11,756 & 10,880 & \\
\hline$s 598$ & 3,5-dimetilfenol & $\mathrm{C}_{8} \mathrm{H}_{10} \mathrm{O}$ & 11,119 & 10,020 & 4,637 & 15,756 & 14,657 & \\
\hline s607 & 2-propilfenol & $\mathrm{C}_{3} \mathrm{H}_{12} \mathrm{O}$ & 12,087 & 10,963 & 5,359 & 17,446 & 16,322 & \\
\hline s.599 & 2-ctilfenol & $\mathrm{C}_{8} \mathrm{H}_{10} \mathrm{O}$ & 10,922 & 9,873 & 4,637 & 15,559 & 14,510 & \\
\hline s606 & 3,4,5-trimetilfenol & $\mathrm{C}_{3} \mathrm{H}_{12} \mathrm{O}$ & 12,528 & 11,264 & 5,359 & 17,887 & 16,623 & \\
\hline s615 & 4-tert-butilfenol & $\mathrm{C}_{10} \mathrm{H}_{14} \mathrm{O}$ & 13,140 & 11,947 & 6,081 & 19,221 & 18,028 & \\
\hline 5605 & 2,3,6-trimetilfenol & $\mathrm{C}_{3} \mathrm{H}_{12} \mathrm{O}$ & 12,520 & 11,265 & 5,359 & 17,879 & 16,624 & \\
\hline$\$ 609$ & 4-propilfenol & $\mathrm{C}_{43} \mathrm{H}_{12} \mathrm{O}$ & 12,221 & 11,041 & 5,359 & 17,580 & 16,400 & \\
\hline s604 & 2.4.6-trimetilfenol & $\mathrm{C}_{3}, \mathrm{H}_{12} \mathrm{O}$ & 12,560 & 11,304 & 5,359 & 17,919 & 16,663 & \\
\hline $\mathrm{s} 600$ & 3-etilfenol & $\mathrm{C}_{8} \mathrm{H}_{10} \mathrm{O}$ & 10,889 & 9,861 & 4,637 & 15,526 & 14,498 & \\
\hline s603 & 2,4,5-trimetilfenol & $\mathrm{C}_{9} \mathrm{H}_{12} \mathrm{O}$ & 12,658 & 11,333 & 5,359 & 18,017 & 16,692 & \\
\hline s602 & 2,3,5-trimetilfenol & $\mathrm{C}_{9} \mathrm{H}_{12} \mathrm{O}$ & 12,551 & 11,282 & 5,359 & 17,910 & 16,641 & \\
\hline
\end{tabular}


Tabela 5.4 (continuação)

\begin{tabular}{|c|c|c|c|c|c|c|c|c|}
\hline No & Soluto & Fórmula & $\alpha_{D}\left(A^{3}\right)$ & $\alpha_{0}\left(\AA^{3}\right)$ & $\Sigma \alpha_{i}\left(\AA^{3}\right)$ & $\alpha_{\text {calc (D) }}\left(A^{3}\right)$ & $\alpha_{\text {calc (o) }}\left(A^{3}\right)$ & $\alpha_{\exp }\left(\AA^{3}\right)$ \\
\hline$\overline{s 601}$ & 4-etilfenol & $\mathrm{C}_{8} \mathrm{H}_{10} \mathrm{O}$ & 11,020 & 9,921 & 4,637 & 15,657 & 14,558 & \\
\hline s595 & 2,5-dimetilfenol & $\mathrm{C}_{8} \mathrm{H}_{10} \mathrm{O}$ & 11,230 & 10,075 & 4,637 & 15,867 & 14,712 & \\
\hline s614 & 2-tert-butilfenol & $\mathrm{C}_{10} \mathrm{H}_{14} \mathrm{O}$ & 13,007 & 11,864 & 6,081 & 19,088 & 17,946 & \\
\hline s593 & 2,3-dimetilfenol & $\mathrm{C}_{8} \mathrm{H}_{10} \mathrm{O}$ & 11,081 & 9,984 & 4,637 & 15,718 & 14,621 & \\
\hline s613 & 2-sec-butilfenol & $\mathrm{C}_{10} \mathrm{H}_{14} \mathrm{O}$ & 13,030 & 11,879 & 6,081 & 19,111 & 17,960 & \\
\hline s612 & 4-butilfenol & $\mathrm{C}_{10} \mathrm{H}_{14} \mathrm{O}$ & 13,355 & 12,107 & 6,081 & 19,436 & 18,188 & \\
\hline s594 & 2,4-dimetilfenol & $\mathrm{C}_{8} \mathrm{H}_{10} \mathrm{O}$ & 11,258 & 10,084 & 4,637 & 15,895 & 14,721 & \\
\hline s597 & 3,4-dimetilfenol & $\mathrm{C}_{8} \mathrm{H}_{10} \mathrm{O}$ & 11,189 & 10,044 & 4,637 & 15,826 & 14,681 & \\
\hline s610 & 2-isopropilfenol & $\mathrm{C}_{9} \mathrm{H}_{12} \mathrm{O}$ & 11,913 & 10,826 & 5,359 & 17,272 & 16,186 & \\
\hline s596 & 2,6-dimetilfenol & $\mathrm{C}_{8} \mathrm{H}_{10} \mathrm{O}$ & 11,098 & 9,995 & 4,637 & 15,735 & 14,632 & \\
\hline s611 & 4-isopropilfenol & $\mathrm{C}_{9} \mathrm{H}_{12} \mathrm{O}$ & 12,052 & 10,916 & 5,359 & 17,411 & 16,275 & \\
\hline s632 & 3-bromofenol & $\mathrm{C}_{6} \mathrm{H}_{5} \mathrm{OBr}$ & 10,560 & 9,384 & 5,222 & 15,783 & 14,606 & \\
\hline s633 & 4-bromofenol & $\mathrm{C}_{6} \mathrm{H}_{5} \mathrm{OBr}$ & 10,535 & 9,356 & 5,222 & 15,757 & 14,578 & \\
\hline s634 & 2-iodofenol & $\mathrm{C}_{6} \mathrm{H}_{5} \mathrm{OI}$ & 11,452 & 10,049 & 7,057 & 18,509 & 17,106 & \\
\hline s635 & 3-iodofenol & $\mathrm{C}_{6} \mathrm{H}_{5} \mathrm{OI}$ & 11,711 & 10,245 & 7,057 & 18,768 & 17,303 & \\
\hline s636 & 4-iodofenol & $\mathrm{C}_{6} \mathrm{H}_{5} \mathrm{OI}$ & 11,680 & 10,204 & 7,057 & 18,737 & 17,261 & \\
\hline s631 & 2-bromofenol & $\mathrm{C}_{6} \mathrm{H}_{5} \mathrm{OBr}$ & 10,364 & 9,231 & 5,222 & 15,586 & 14,454 & \\
\hline s638 & 3-metoxifenol & $\mathrm{C}_{7} \mathrm{H}_{8} \mathrm{O}_{2}$ & 10,718 & 9,604 & 4,147 & 14,866 & 13,752 & \\
\hline s639 & 4-metoxifenol & $\mathrm{C}_{7} \mathrm{H}_{8} \mathrm{O}_{2}$ & 10,874 & 9,663 & 4,147 & 15,021 & 13,810 & \\
\hline s640 & 2-cianofenol & $\mathrm{C}_{7} \mathrm{H}_{5} \mathrm{NO}$ & 11,207 & 9,964 & 3,587 & 14,794 & 13,551 & \\
\hline s641 & 3-cianofenol & $\mathrm{C}_{7} \mathrm{H}_{5} \mathrm{NO}$ & 11,194 & 9,969 & 3,587 & 14,781 & 13,557 & \\
\hline s590 & $o$-cresol & $\mathrm{C}_{7} \mathrm{H}_{8} \mathrm{O}$ & 9,747 & 8,771 & 3,915 & 13,662 & 12,686 & \\
\hline s591 & $m$-cresol & $\mathrm{C}_{7} \mathrm{H}_{8} \mathrm{O}$ & 9,743 & 8,772 & 3,915 & 13,658 & 12,687 & 13,05 \\
\hline s592 & $p$-cresol & $\mathrm{C}_{7} \mathrm{H}_{8} \mathrm{O}$ & 9,873 & 8,837 & 3,915 & 13,788 & 12,752 & \\
\hline s637 & 2-metoxifenol & $\mathrm{C}_{7} \mathrm{H}_{8} \mathrm{O}_{2}$ & 10,503 & 9,471 & 4,147 & 14,650 & 13,618 & \\
\hline s630 & 4-cloro-3-metilfenol & $\mathrm{C}_{7} \mathrm{H}_{7} \mathrm{OCl}$ & 11,469 & 10,189 & 5,010 & 16,479 & 15,199 & \\
\hline s629 & 4-cloro-2-metilfenol & $\mathrm{C}_{7} \mathrm{H}_{7} \mathrm{OCl}$ & 11,598 & 10,284 & 5,010 & 16,608 & 15,294 & \\
\hline
\end{tabular}

$\alpha_{\mathrm{D}}$ - polarizabilidade dependente da freqüência - calculada com hamiltonianoMNDO-PM3 para $\lambda=5893 \AA^{3}$ sem correção atômica), $\alpha_{0}$ polarizabilidade estática - calculada com hamiltoniano $M N D O-P M 3 \mathrm{sem}$ correção atômica), $\Sigma \alpha_{i}$ - correção atômica da polarizabilidade

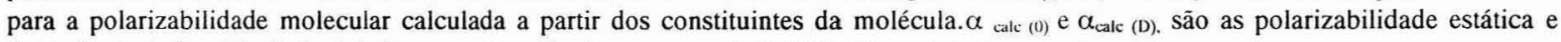
dependente da frequêencia incluindo correçōes atômicas. $\alpha_{\text {cxp }}$ - polarizabilidade molecular experimental.

\subsection{Análise dos Dados}

Considerando as múltiplas possibilidades de calcular os parâmetros teóricos, realizamos exaustivas análises do conjunto de valores obtidos para os parâmetros teóricos com o objetivo de estabelecer um critério de escolha do melhor conjunto de parâmetros que em princípio possibilitasse a obtenção de equações de regressão com significância estatística comparada com as obtidas por LSER. Na Tabela 5.5 mostramos dois conjuntos de valores para os parâmetros volume $(V)$, e cargas formais $\left(q_{+}\right.$e $\left.q_{-}\right)$. As cargas formais foram obtidas diretamente da análise populacional de Mulliken e pela técnica semiempírica CM1. Já os valores do volume são obtidos através dos métodos de Hopfinger e McGowan, ambos calculados a partir da estrutura molecular do soluto. 


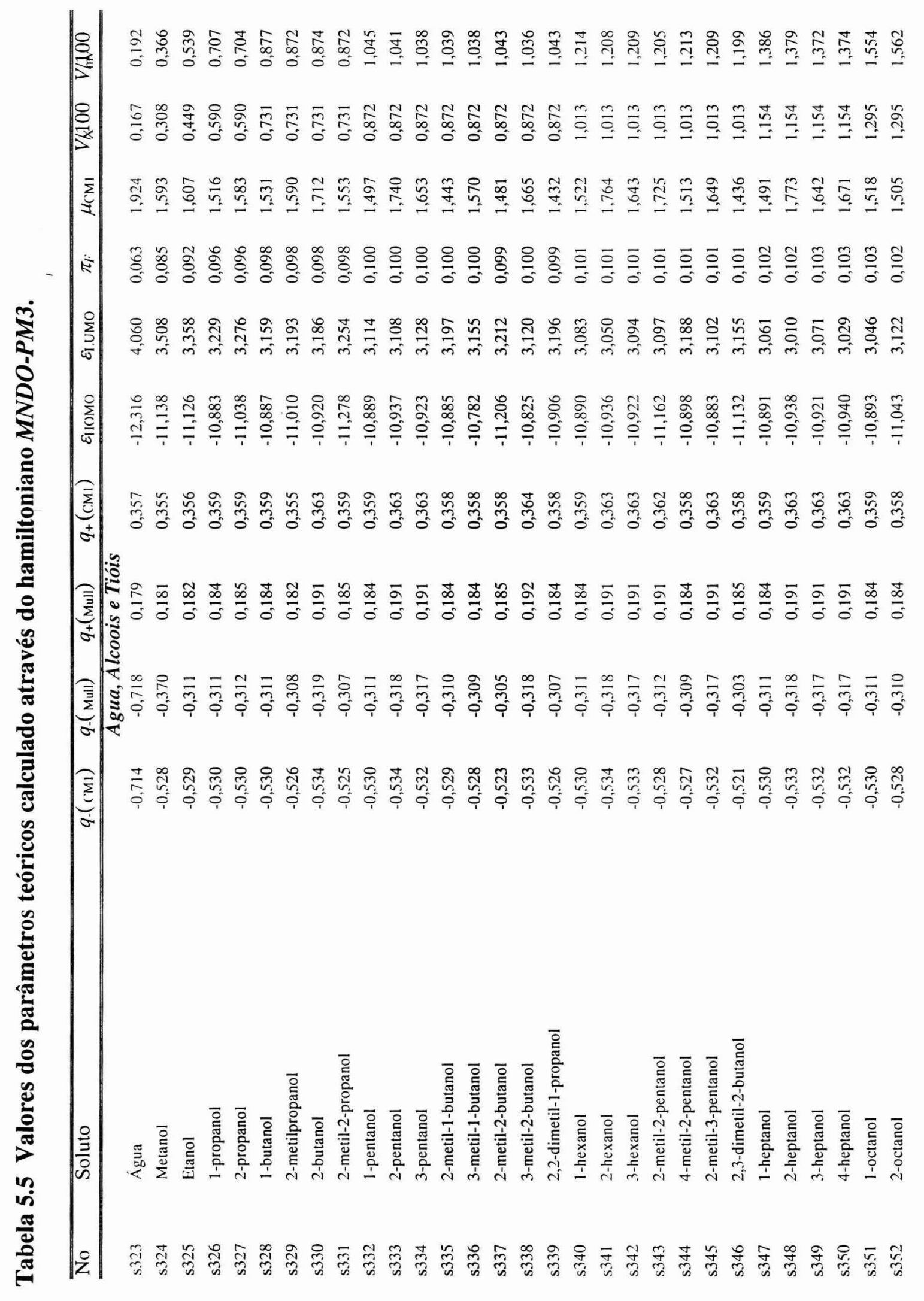




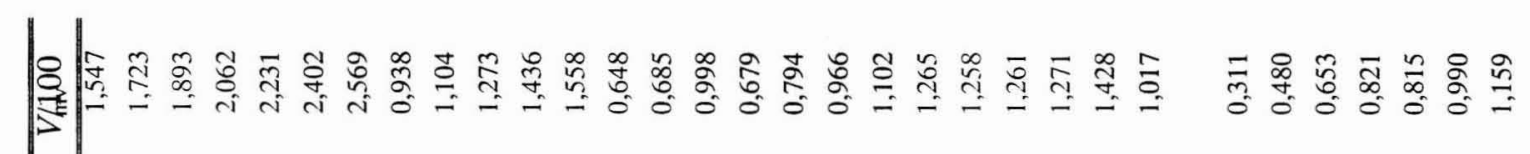

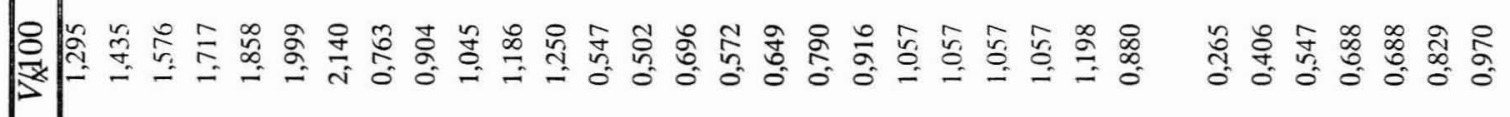

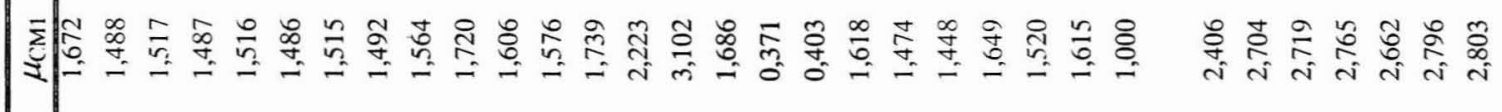

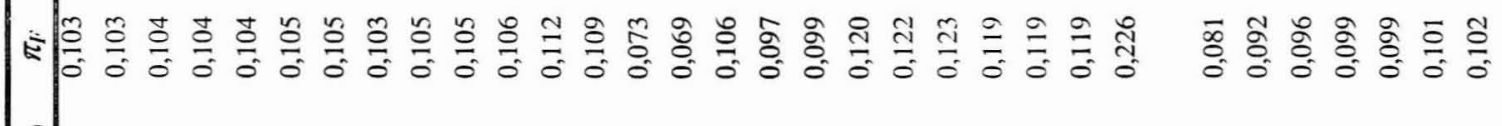

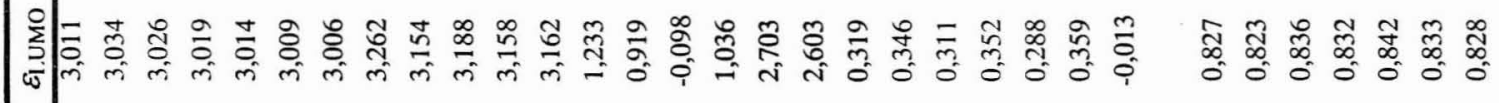

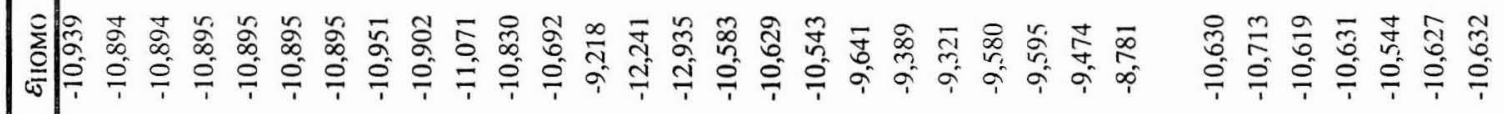

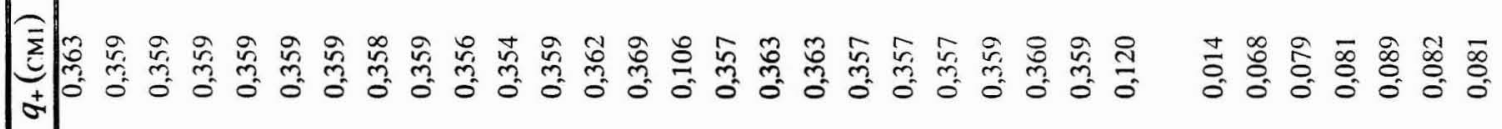

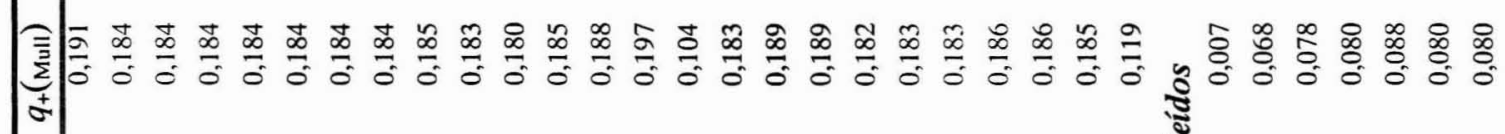

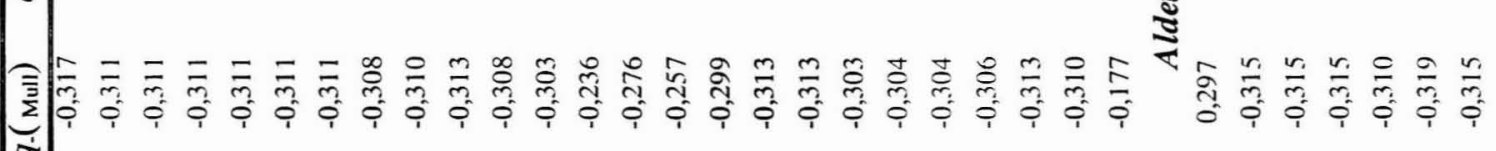

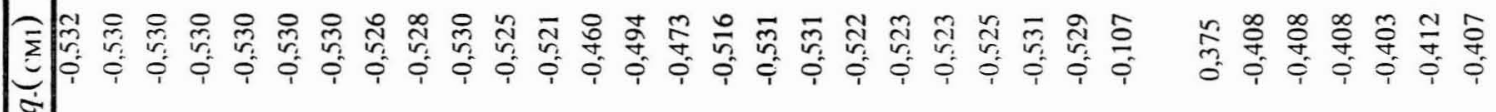

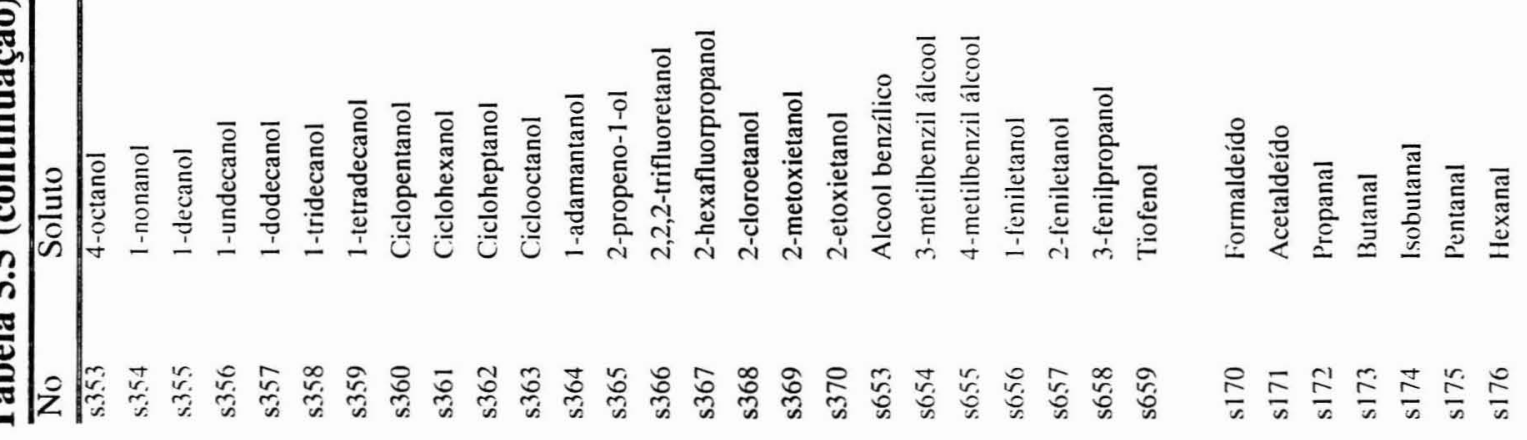




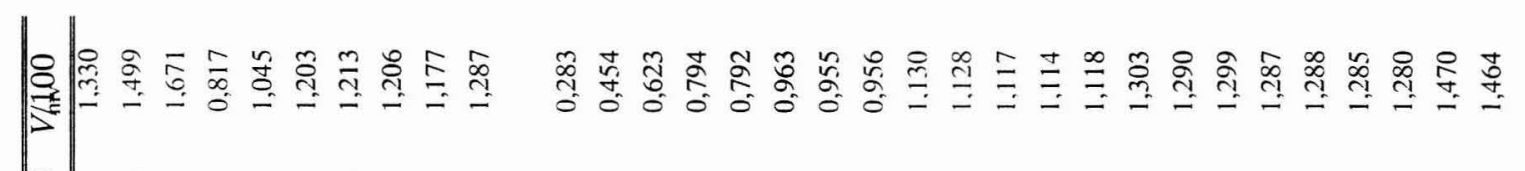

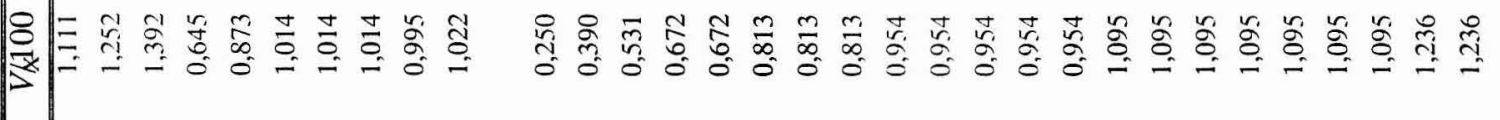

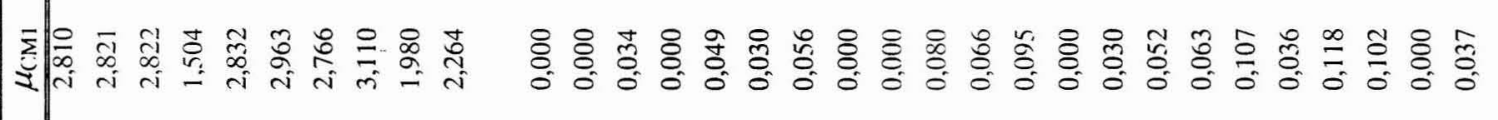

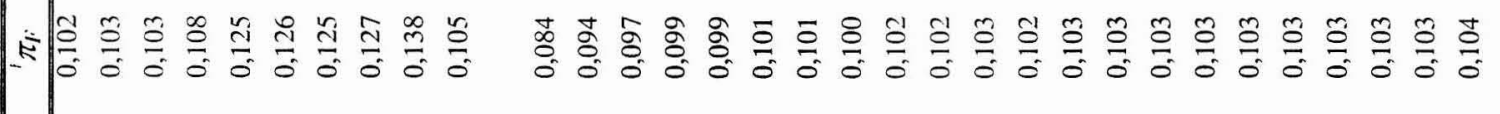

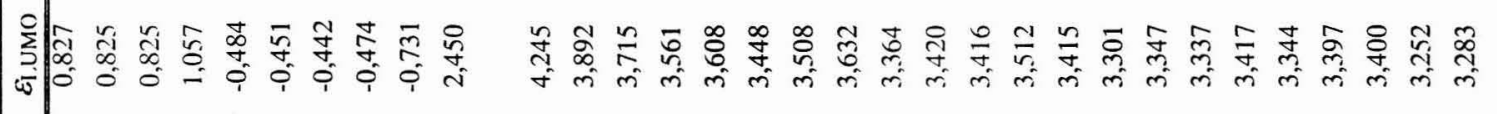

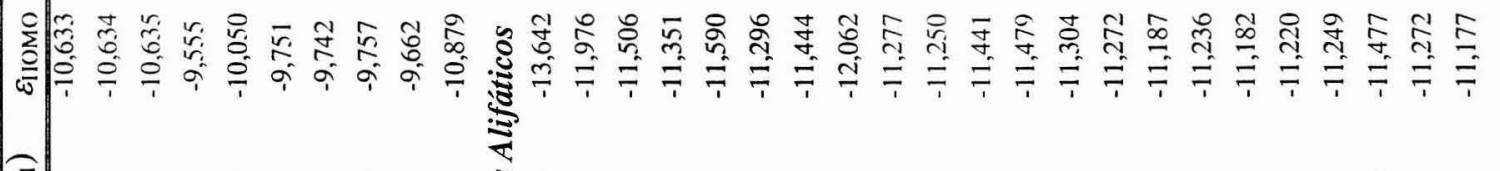

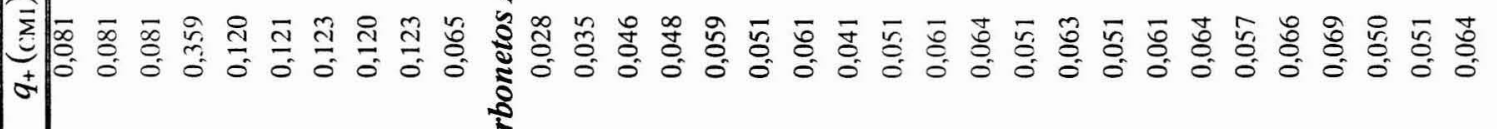

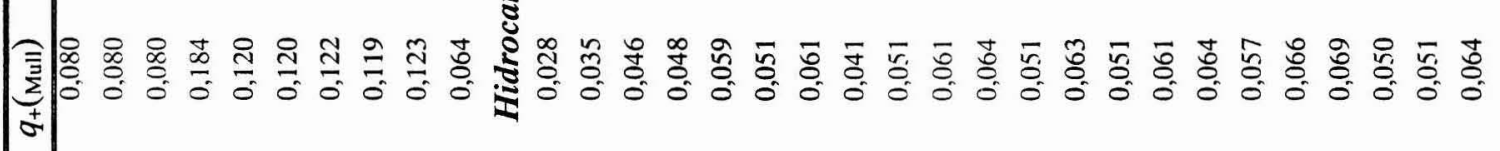

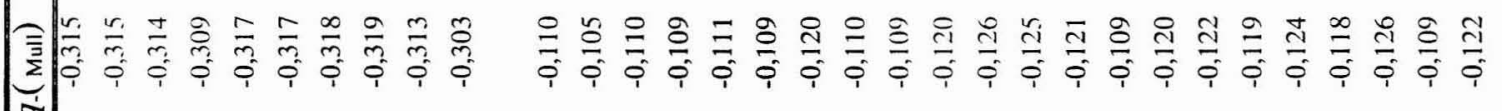

| 


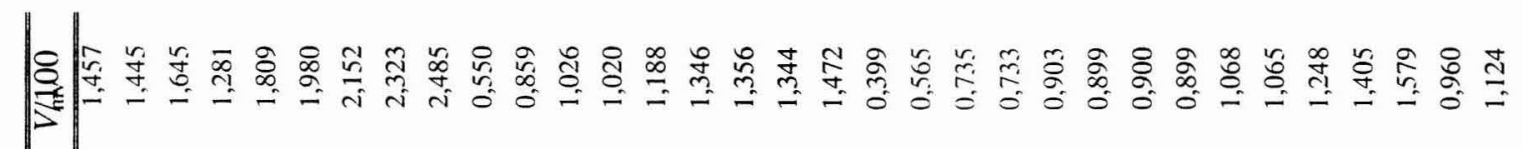

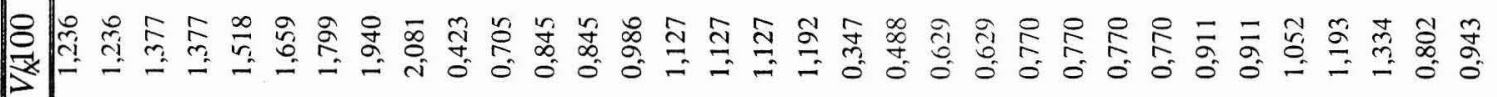

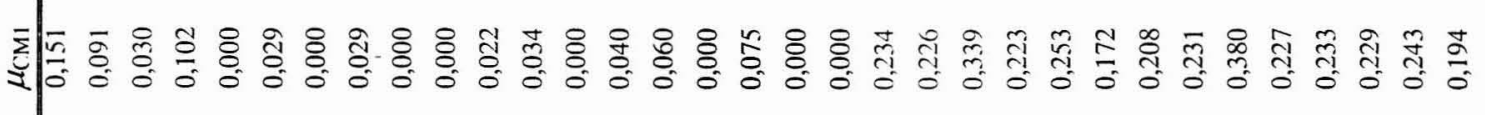

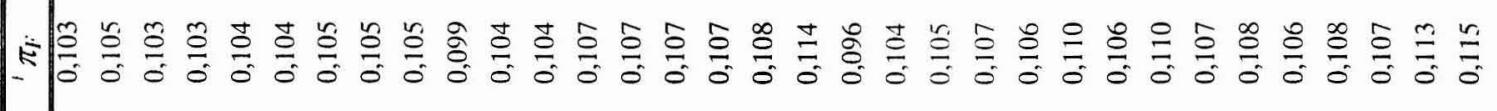

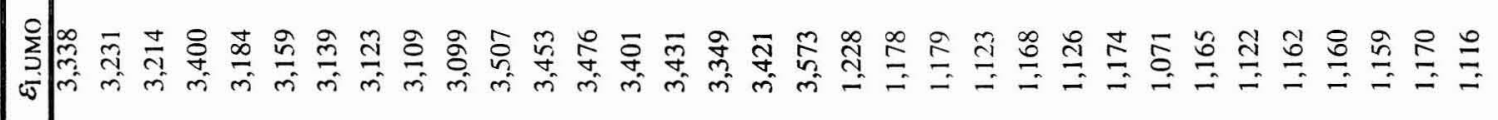

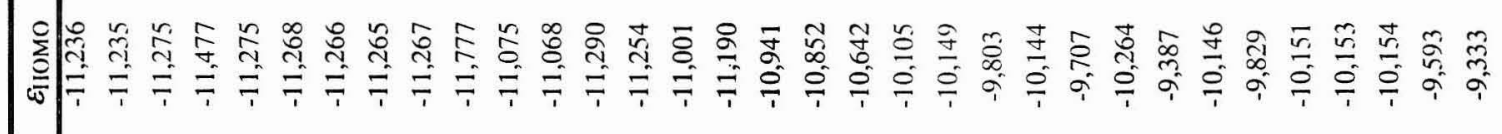

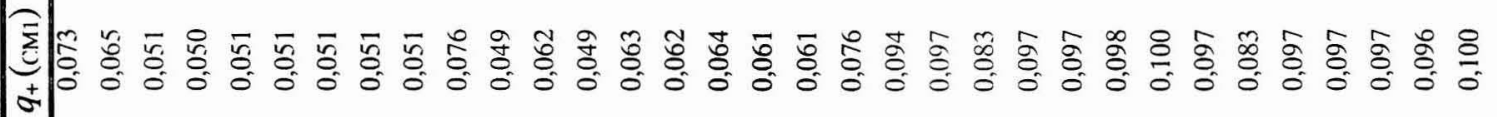

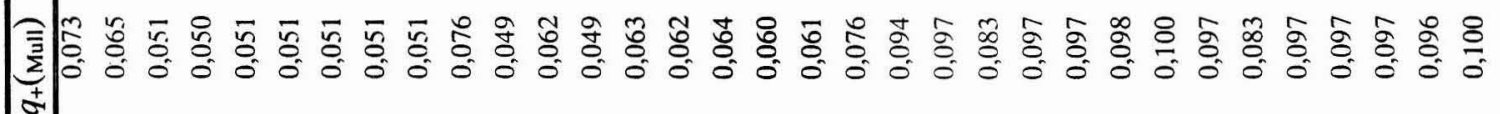

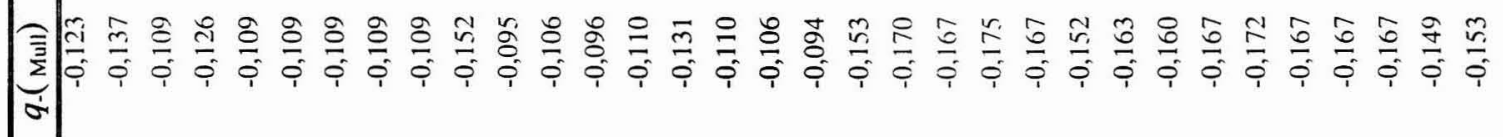

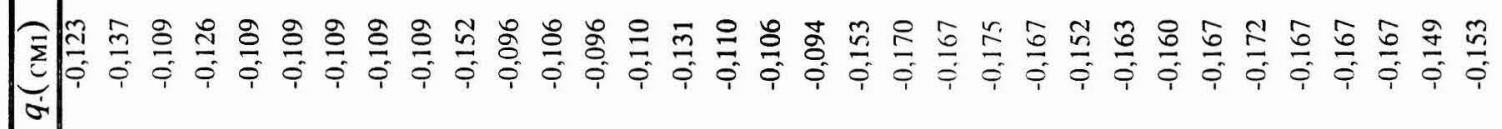

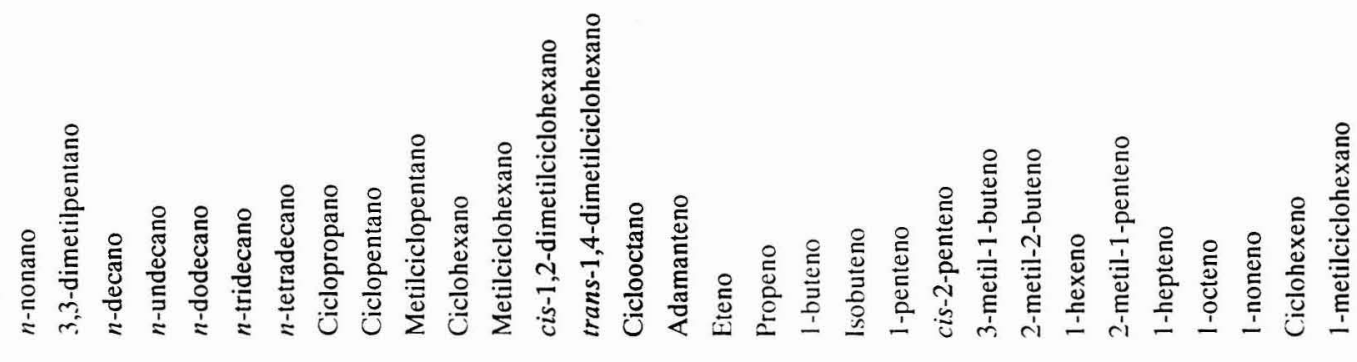

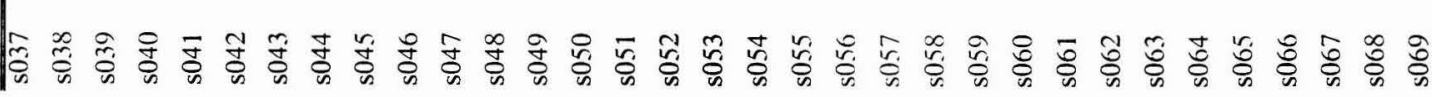




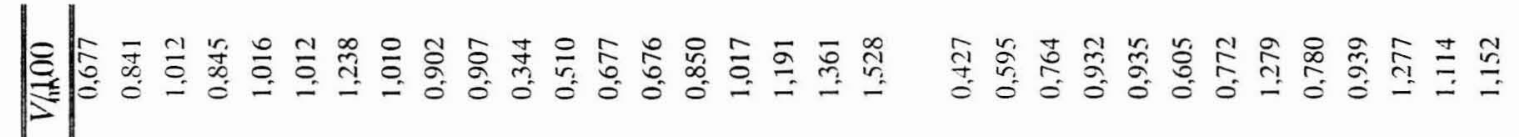

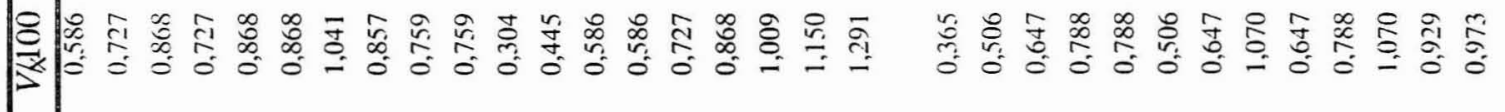

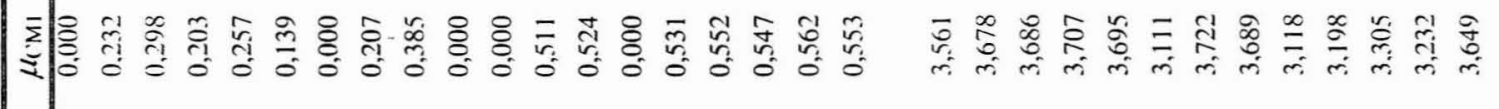

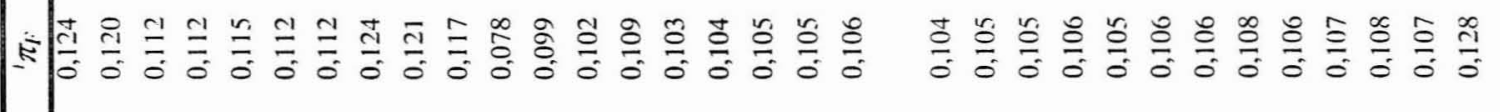

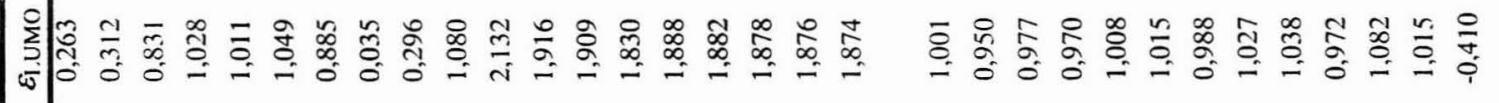

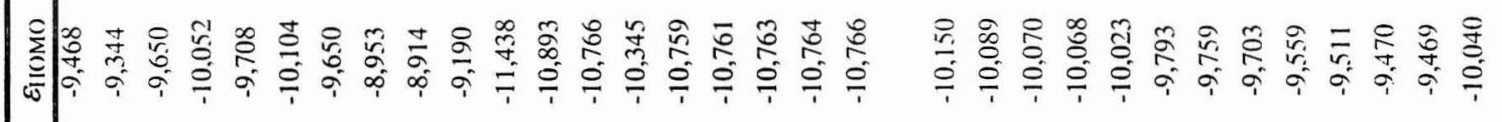

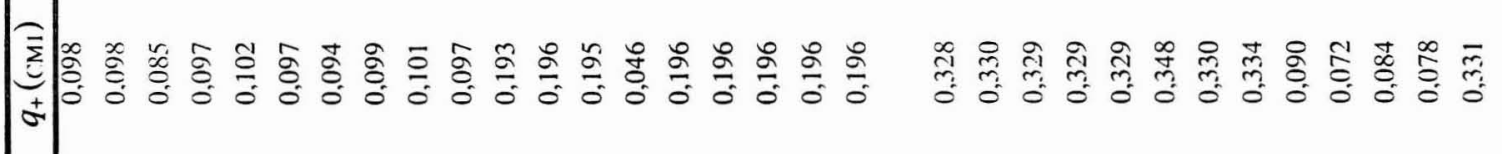

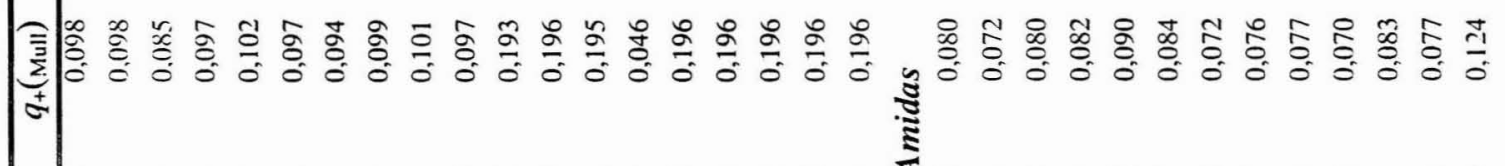

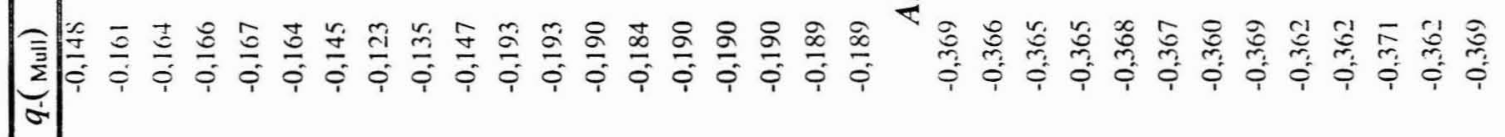

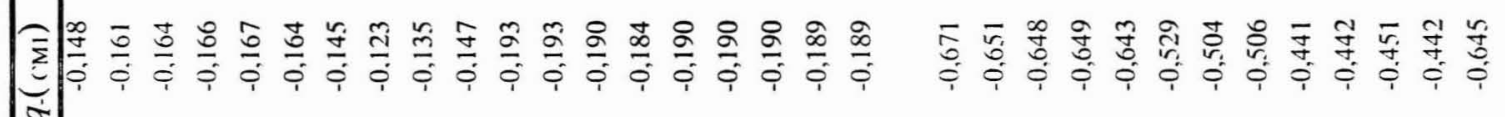

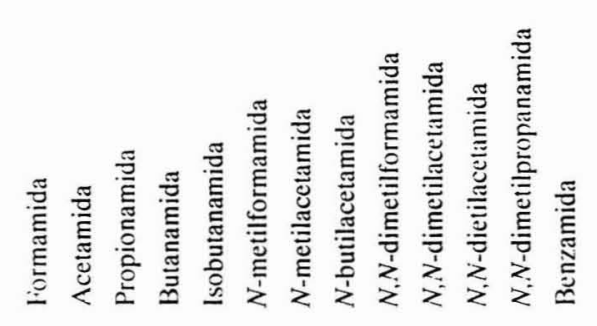

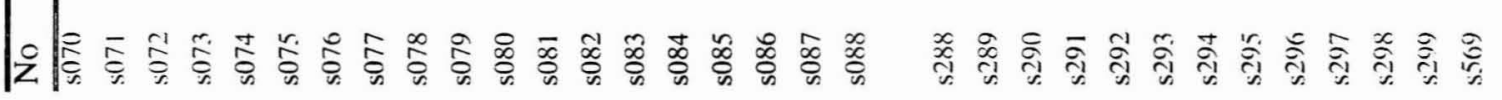




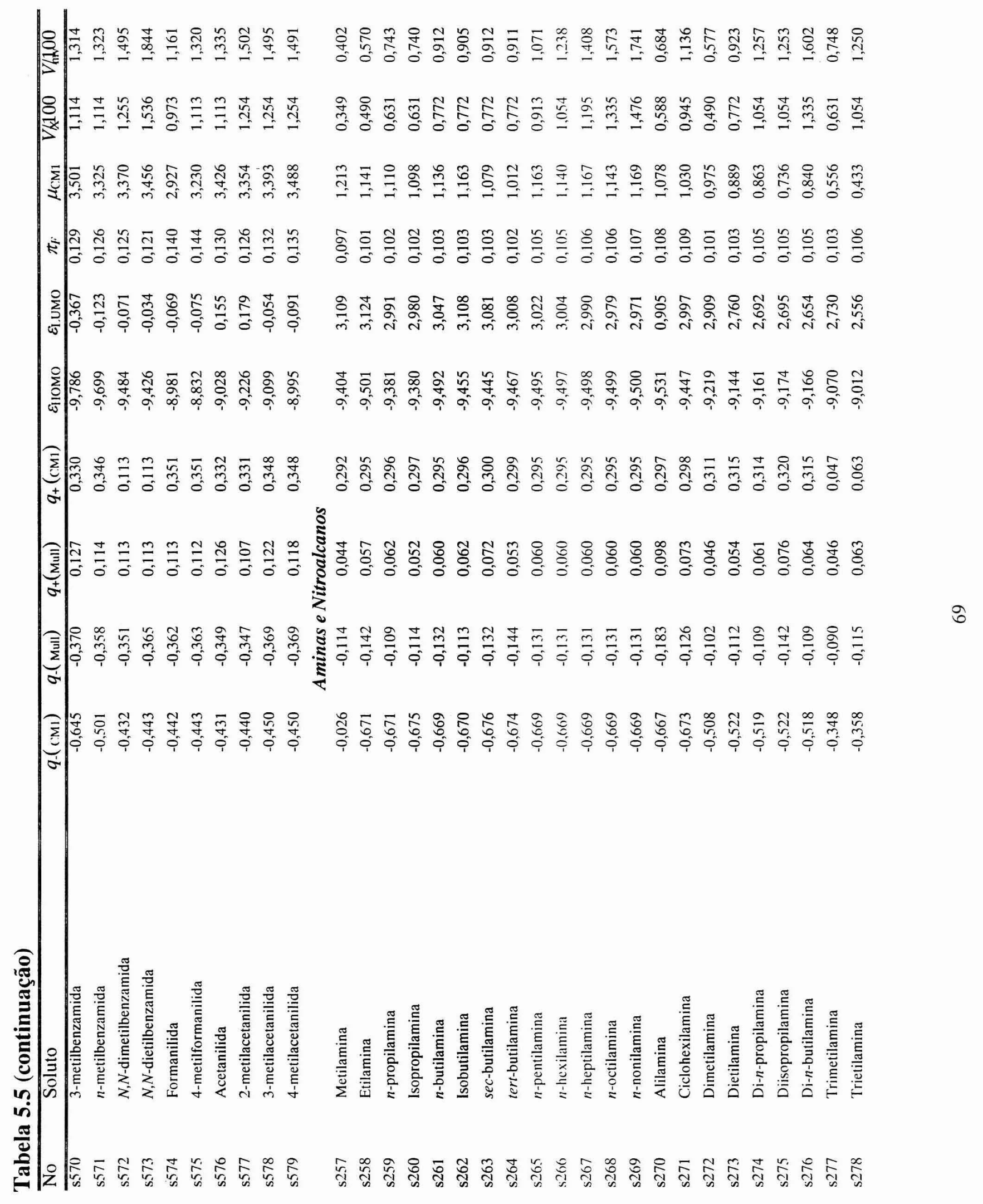




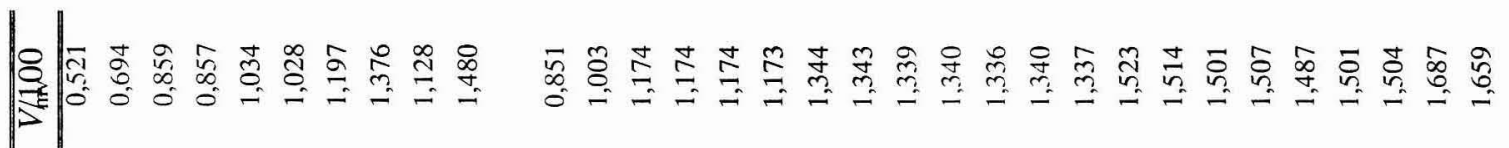

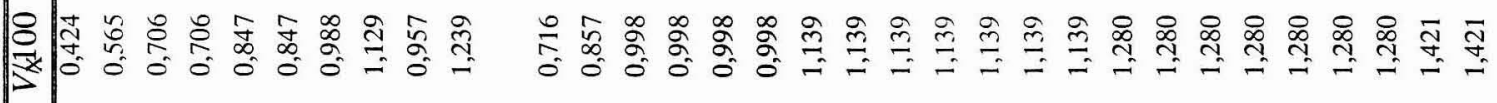

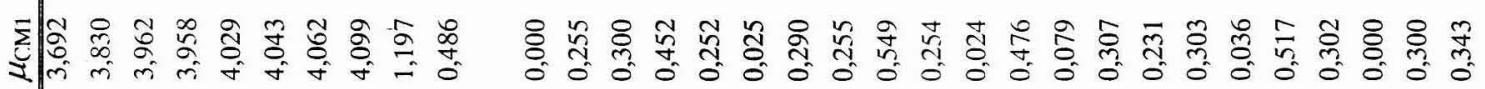

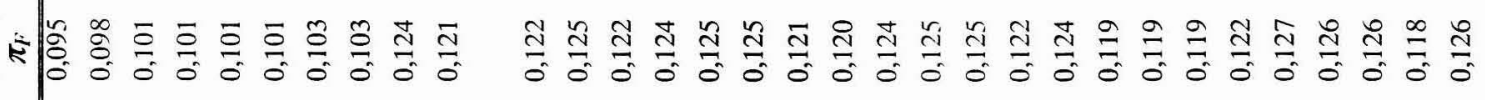

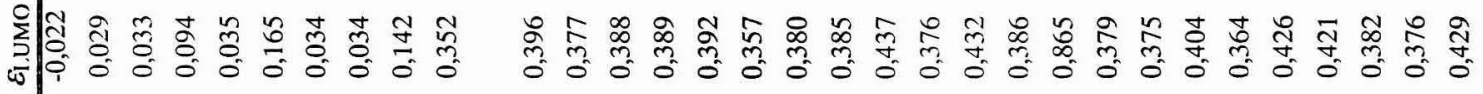

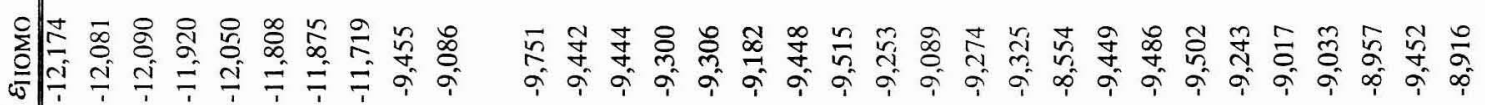

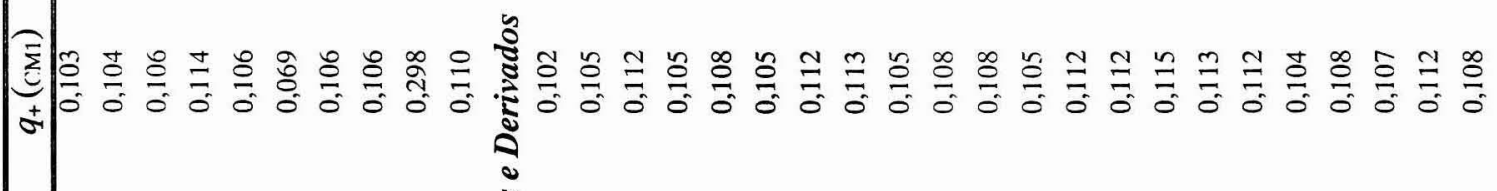

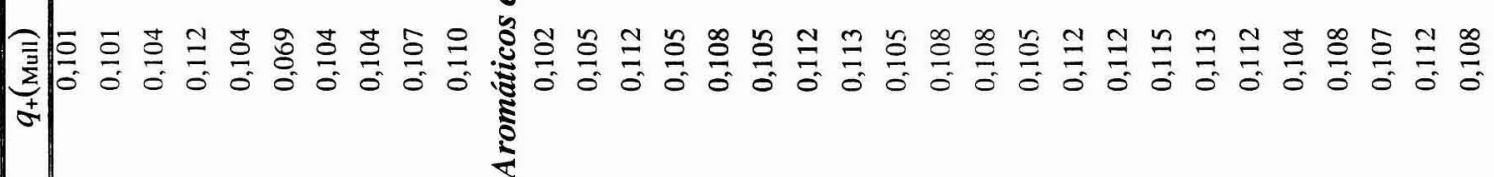

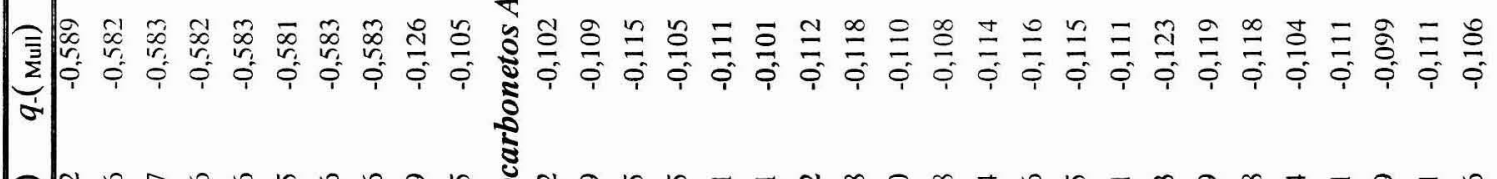

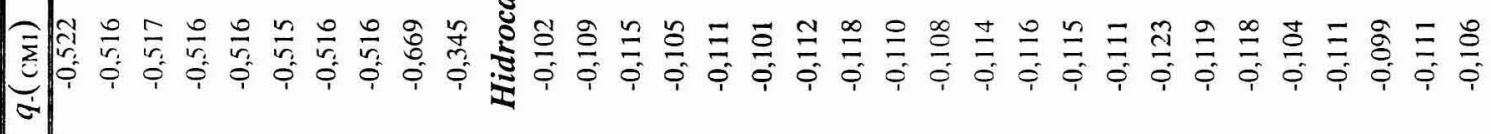

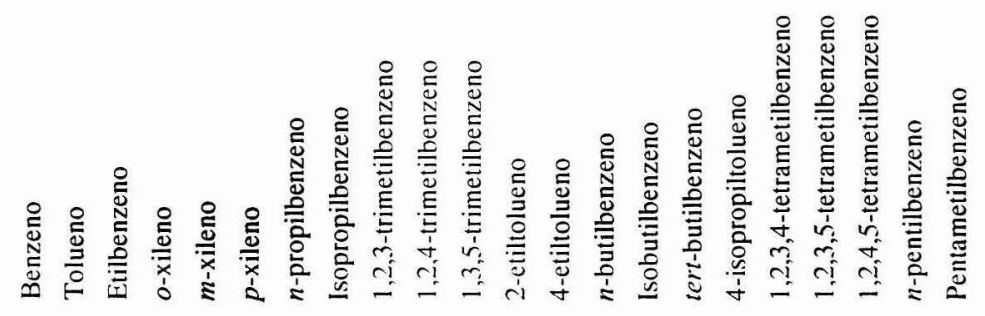

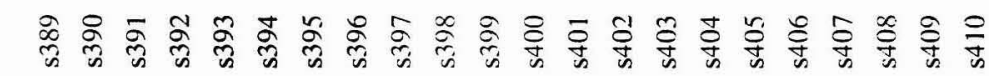




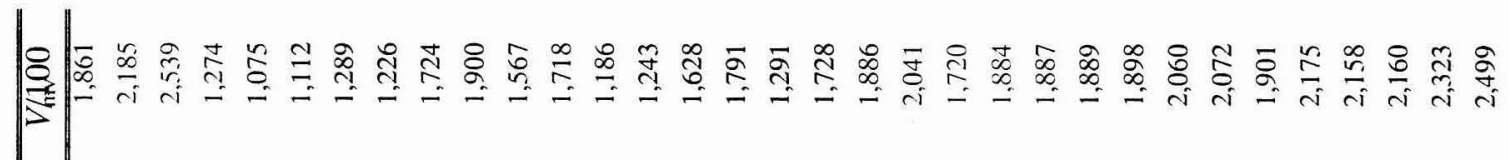

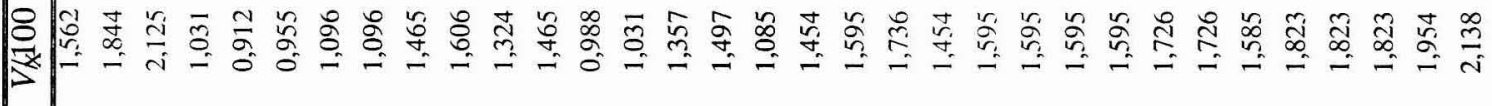

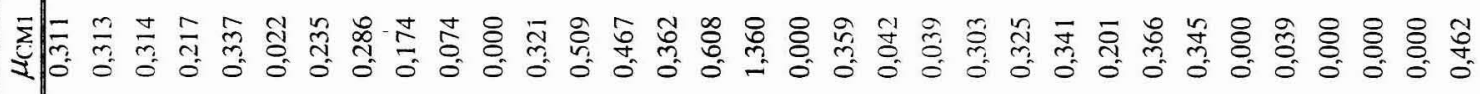

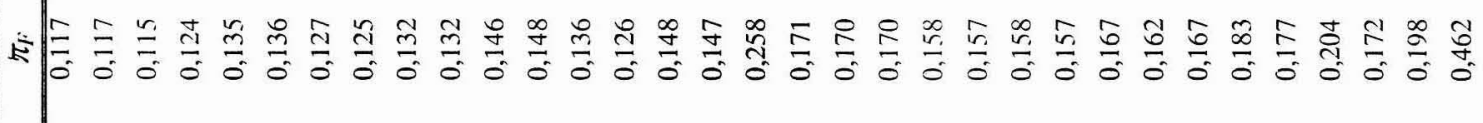

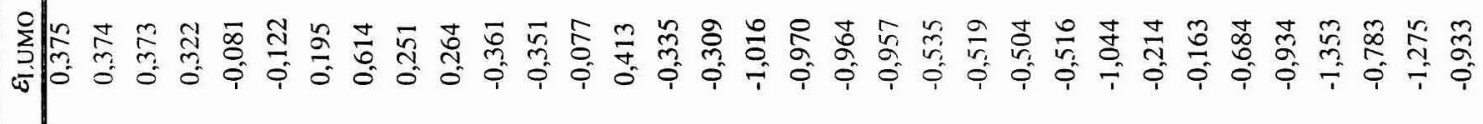

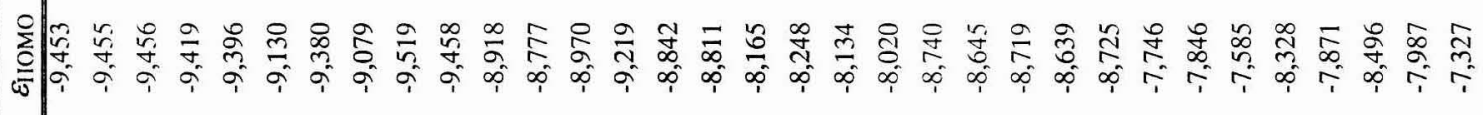

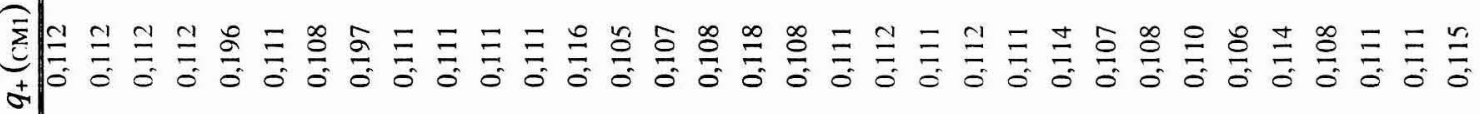

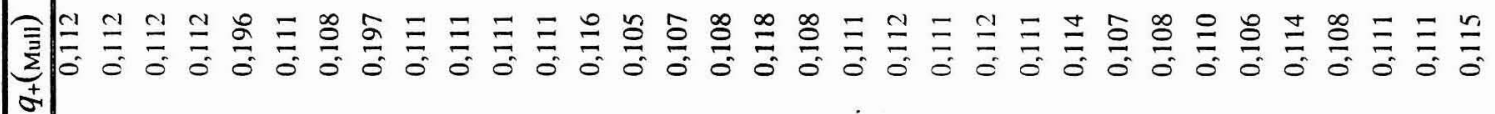

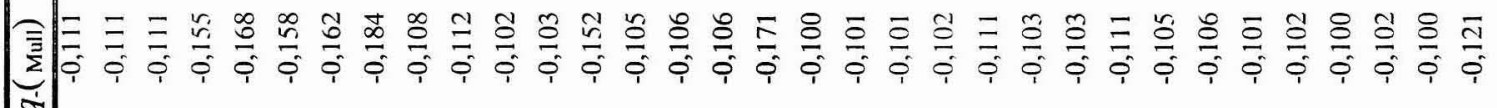

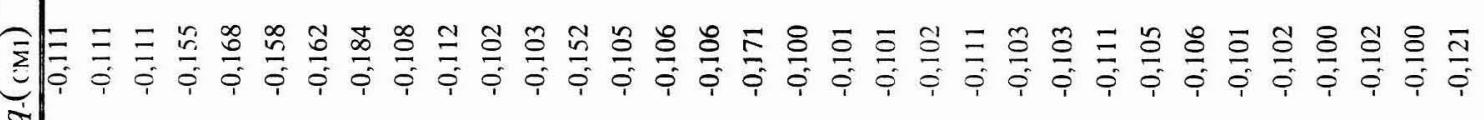

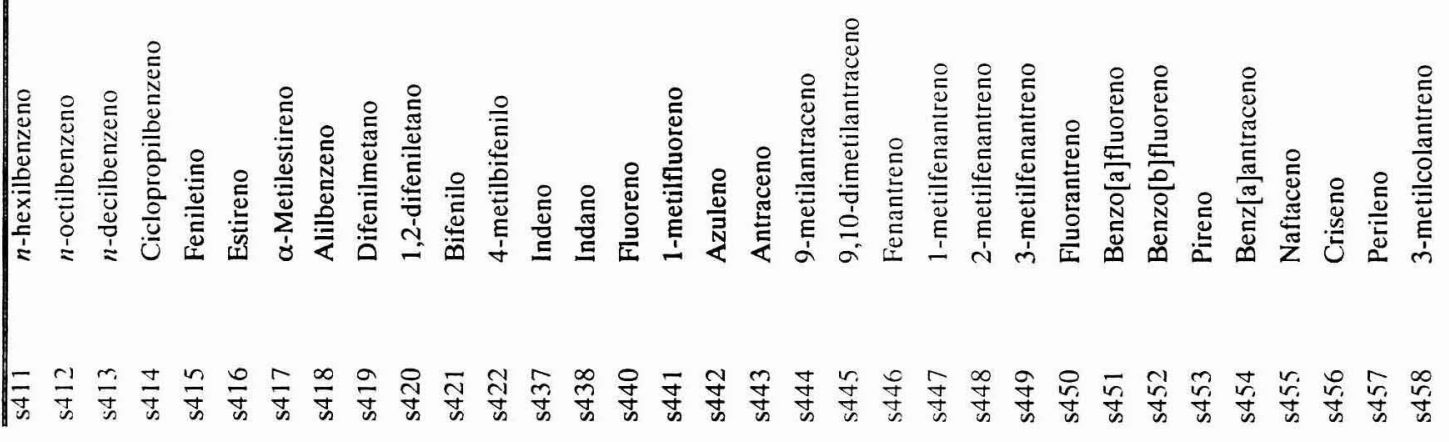




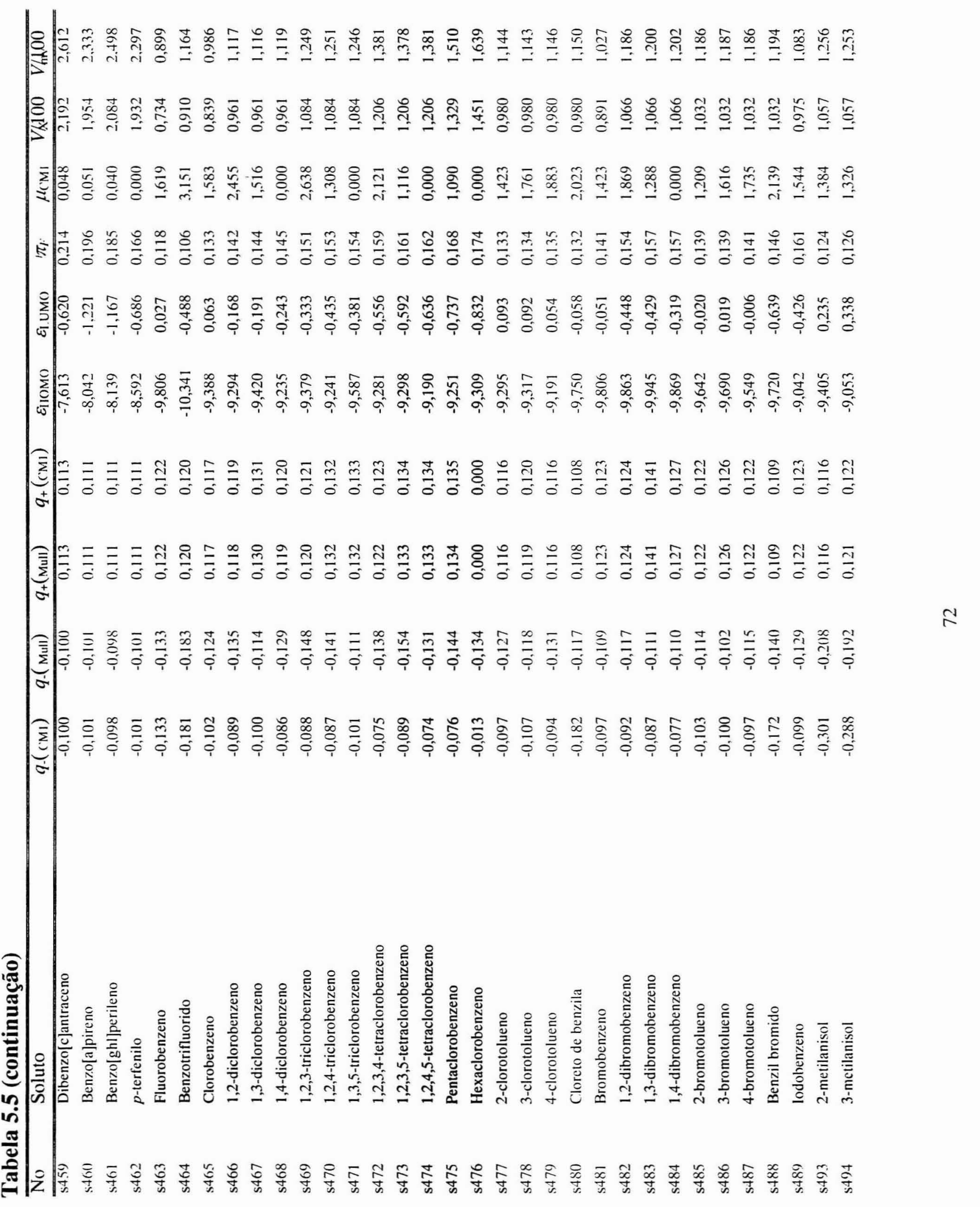




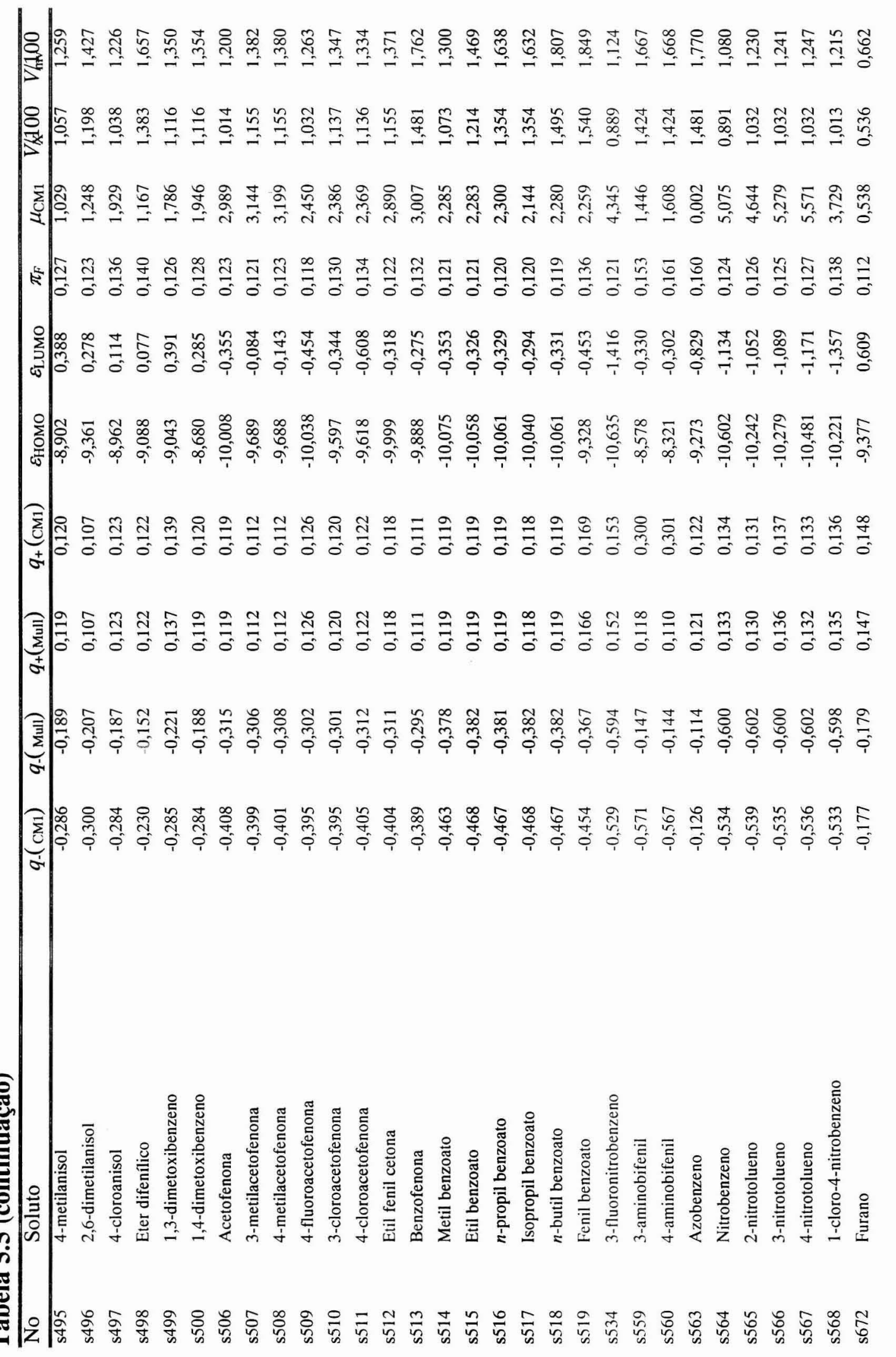




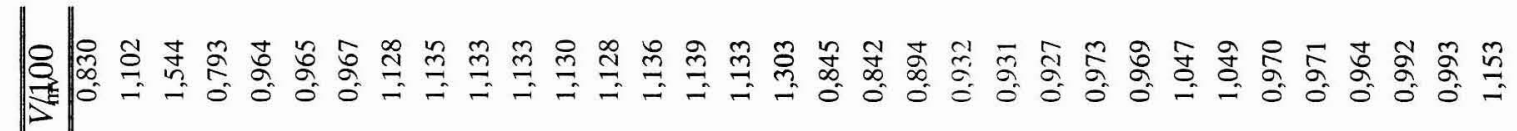

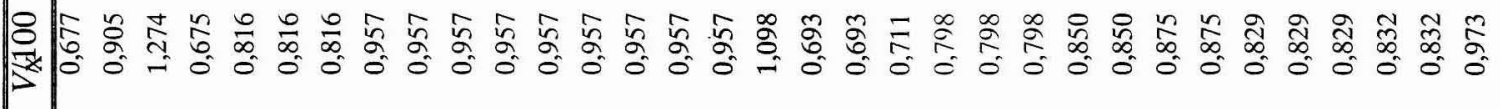

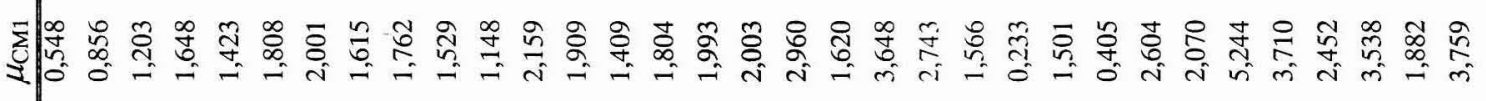

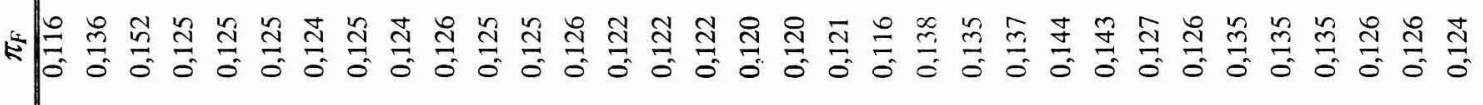

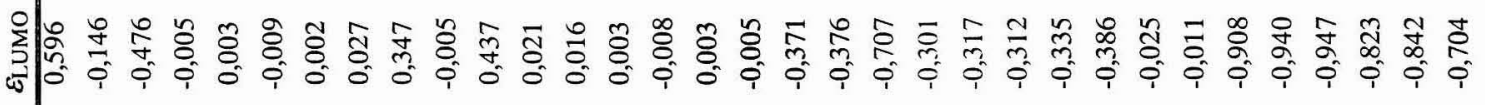

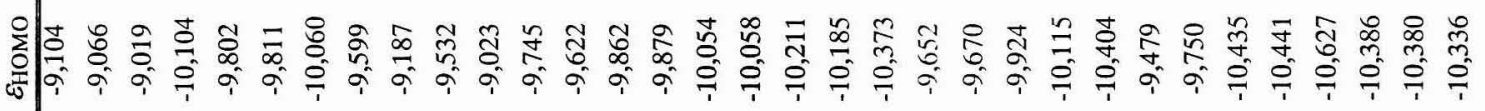

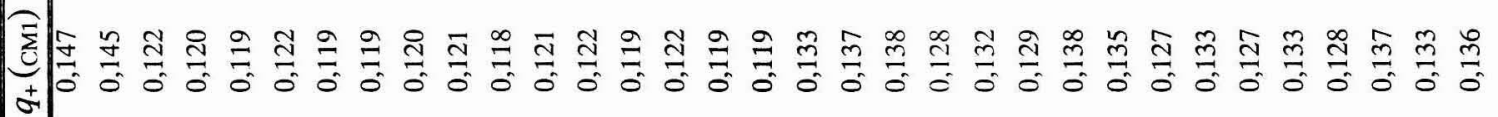

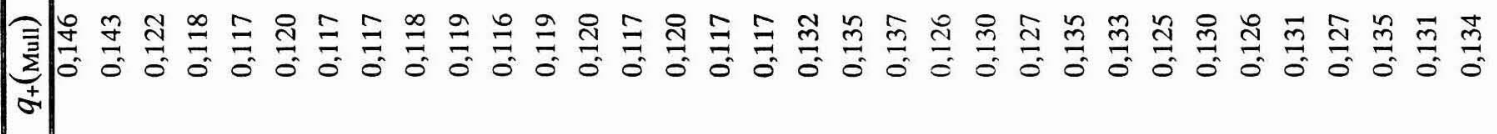

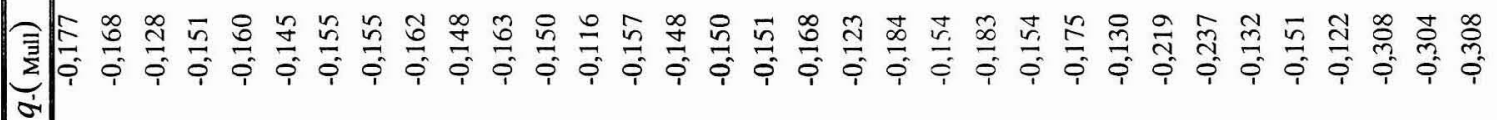

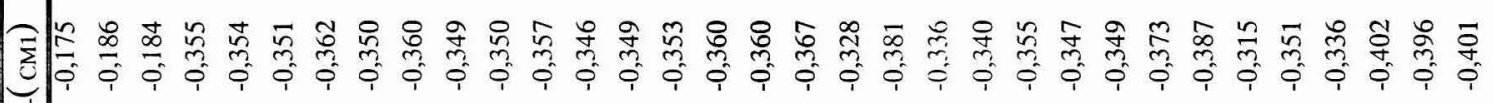




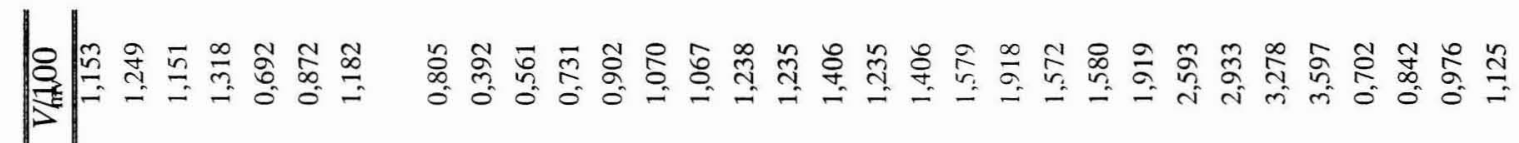

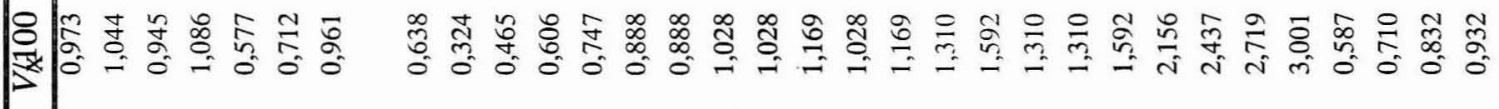

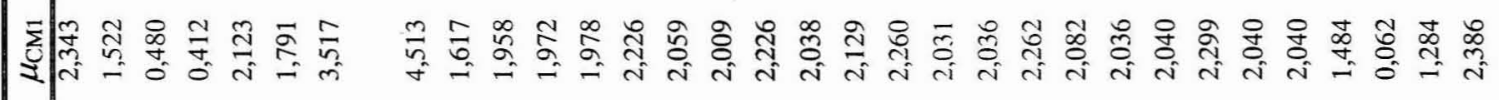

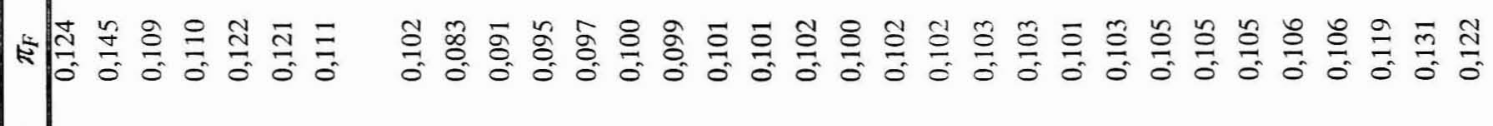

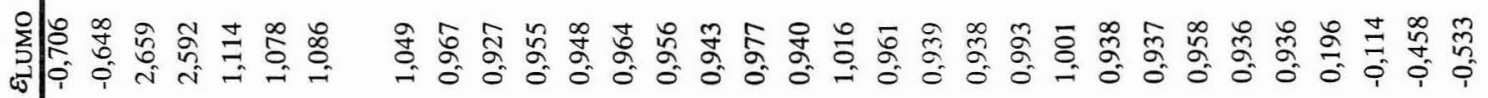

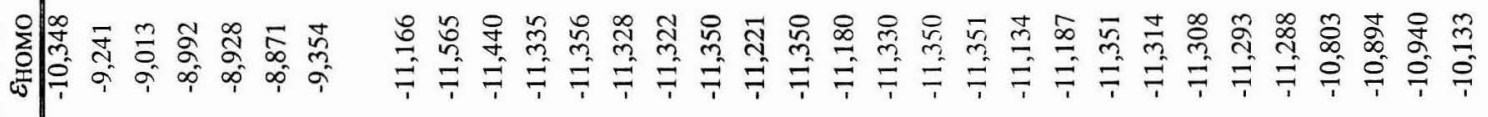

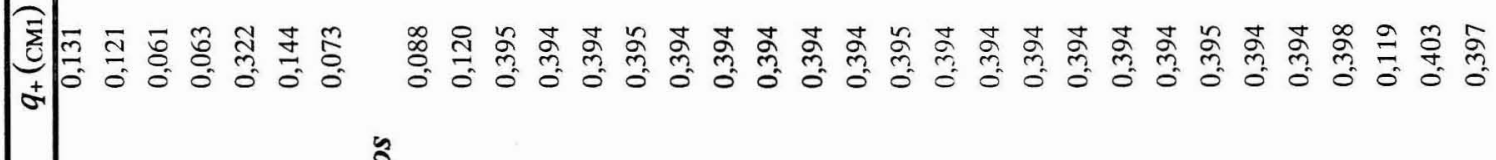

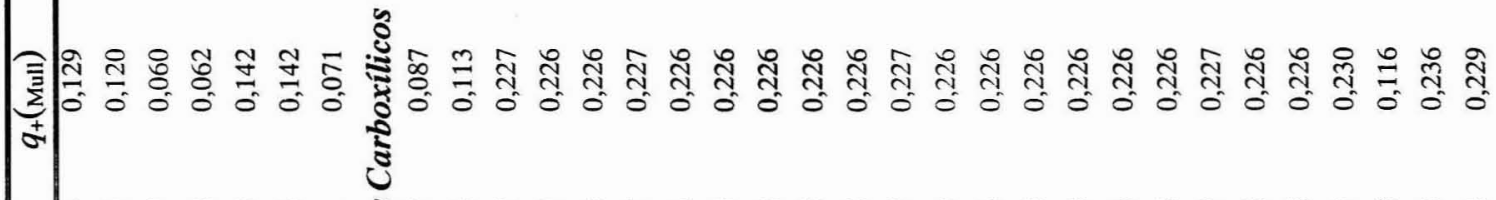

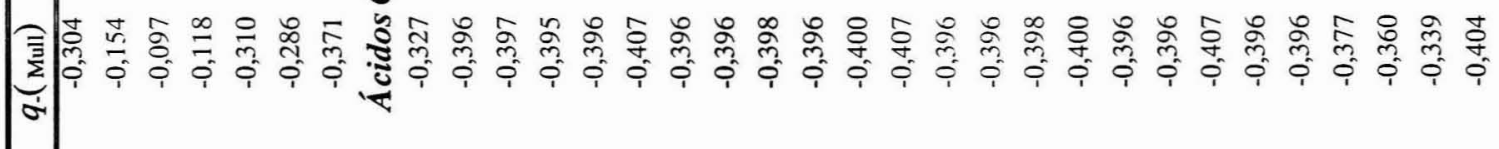

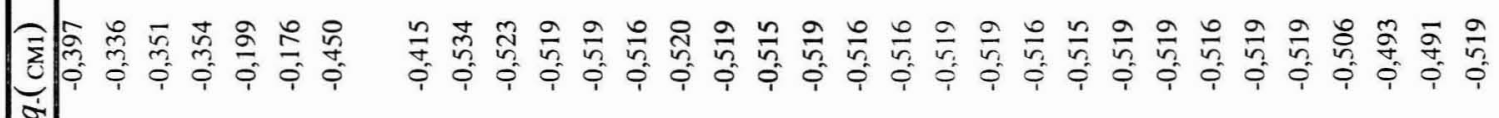

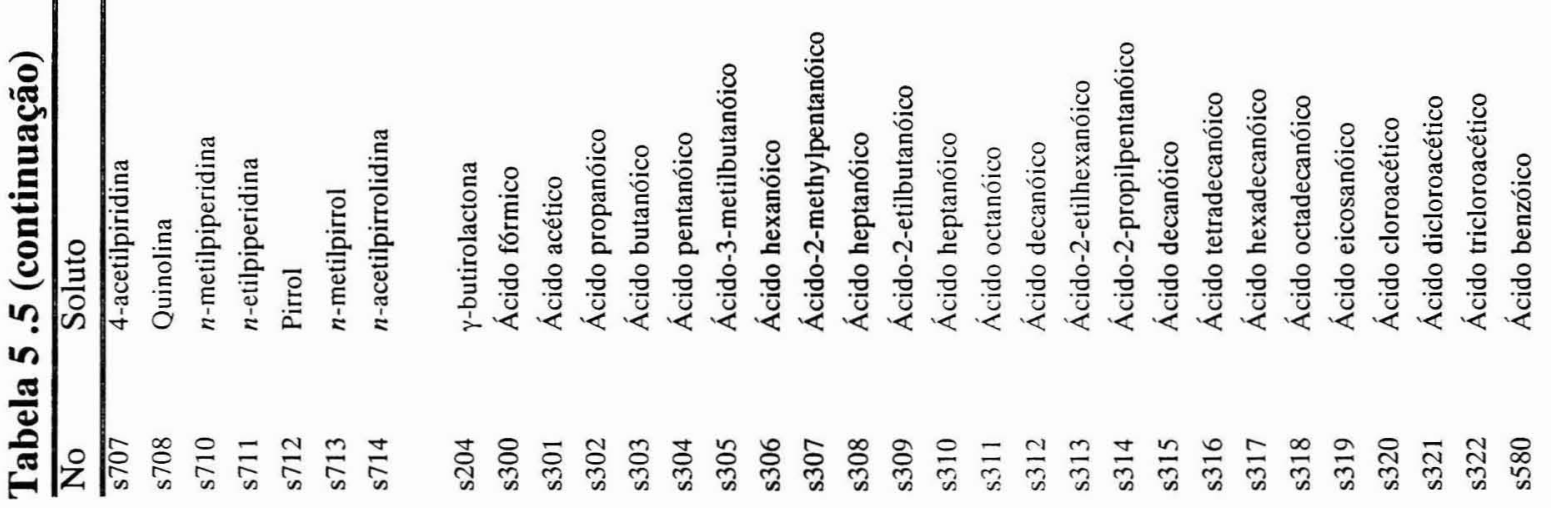




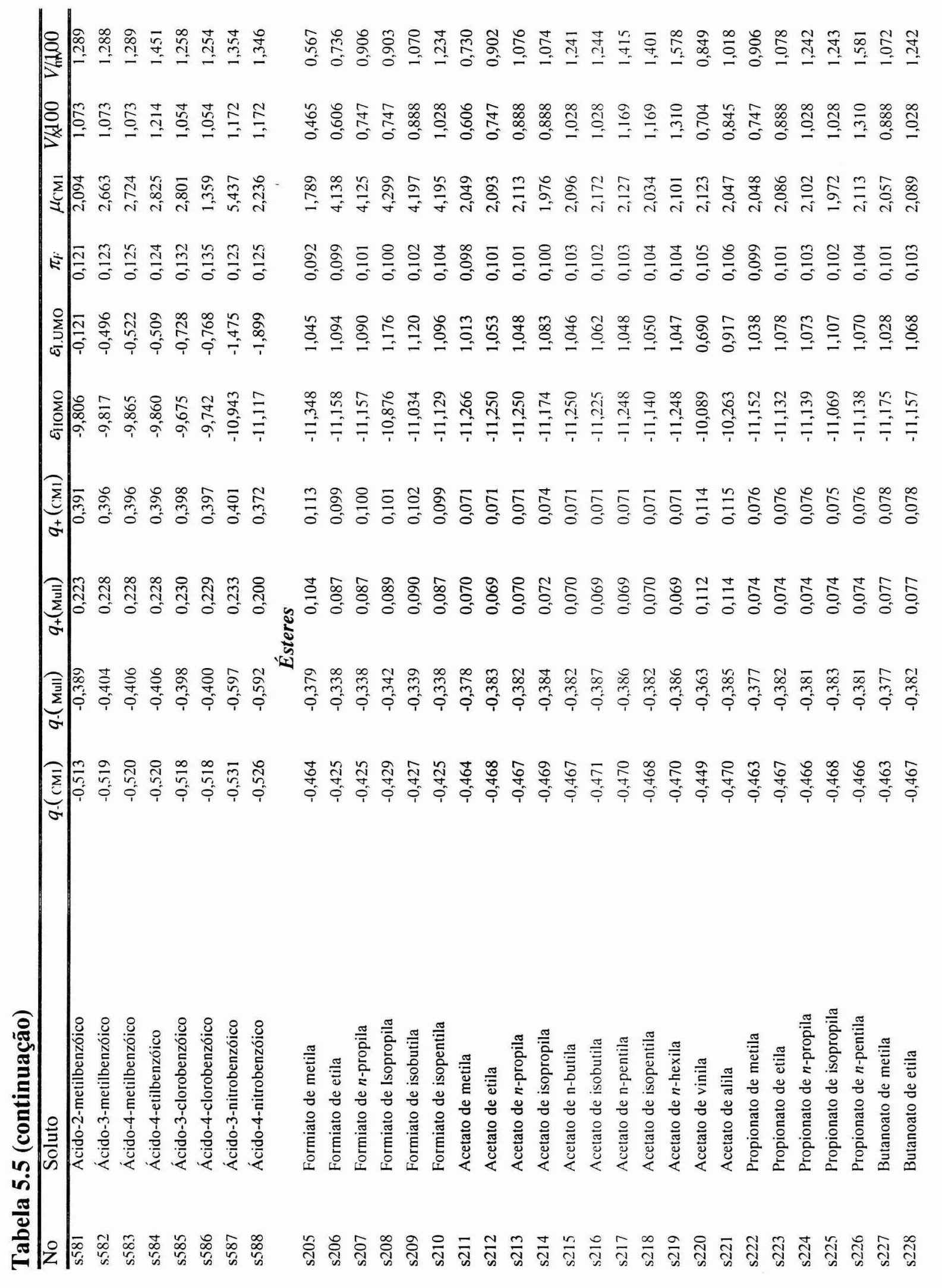




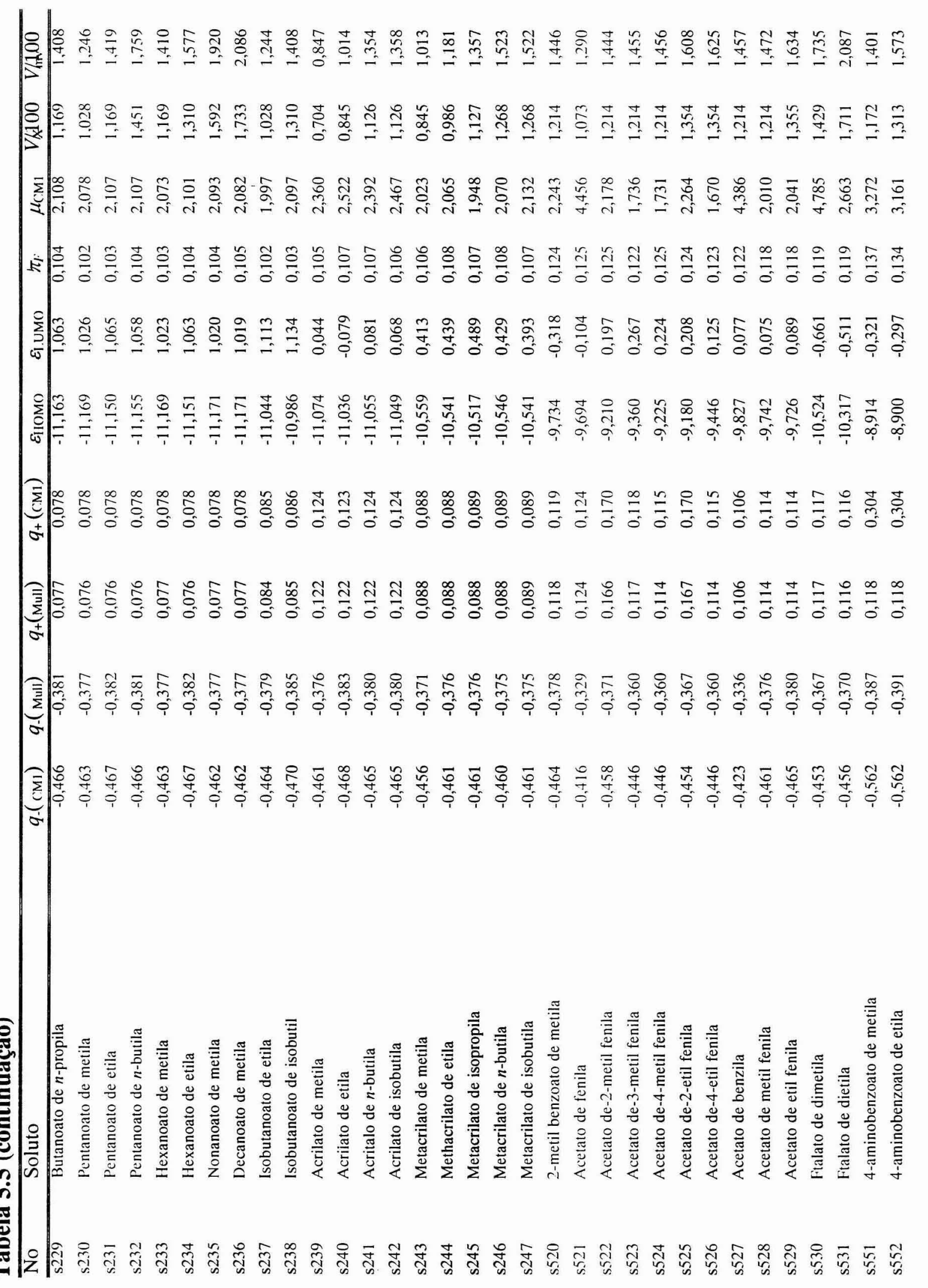




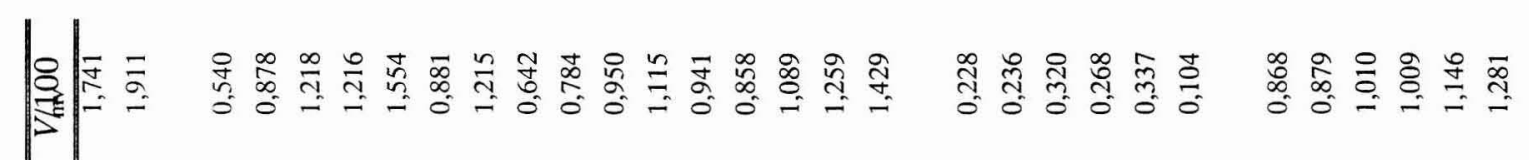

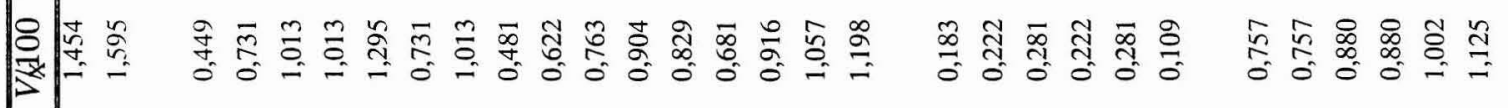

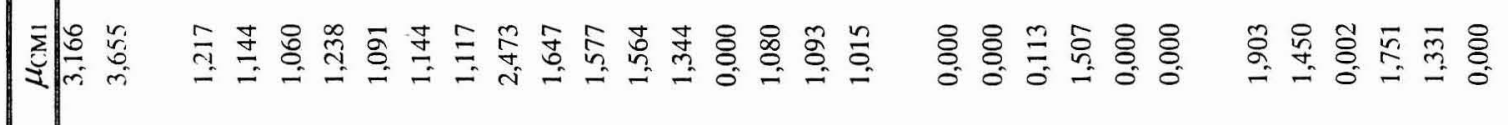

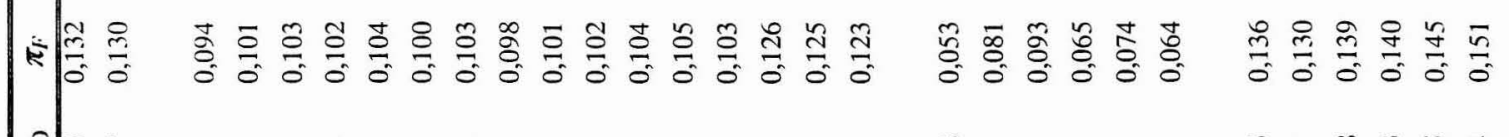

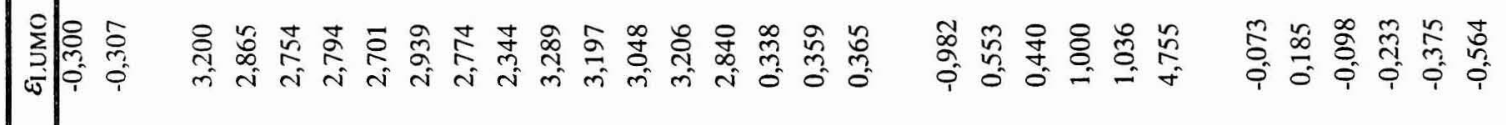

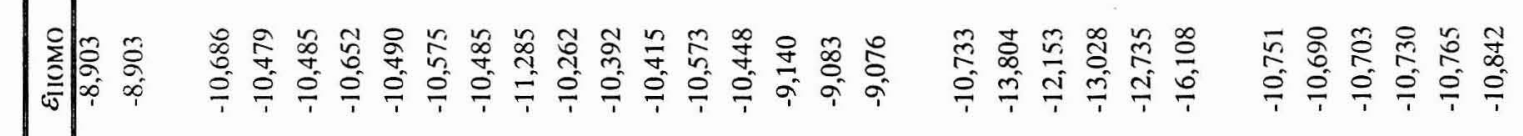

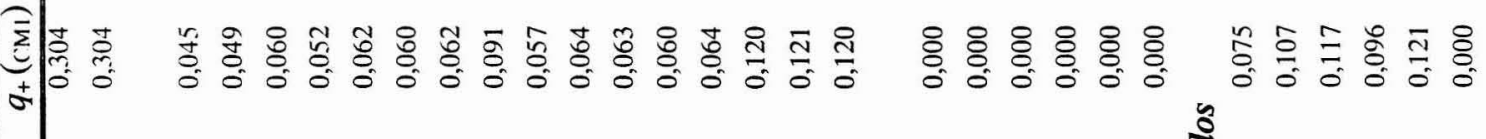

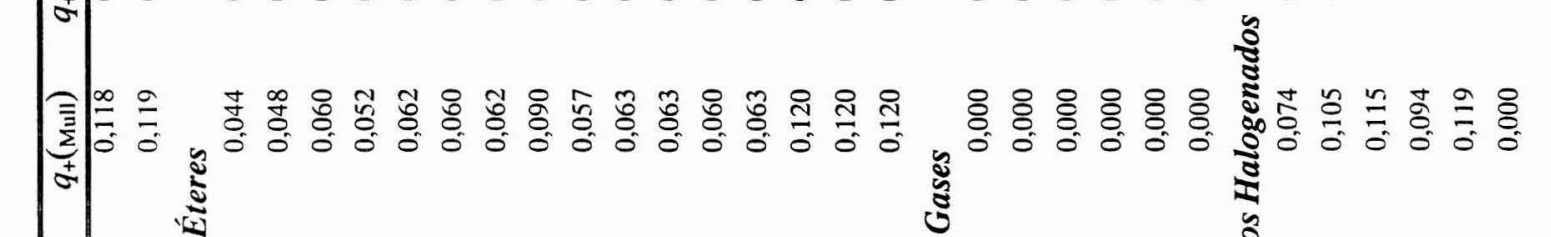

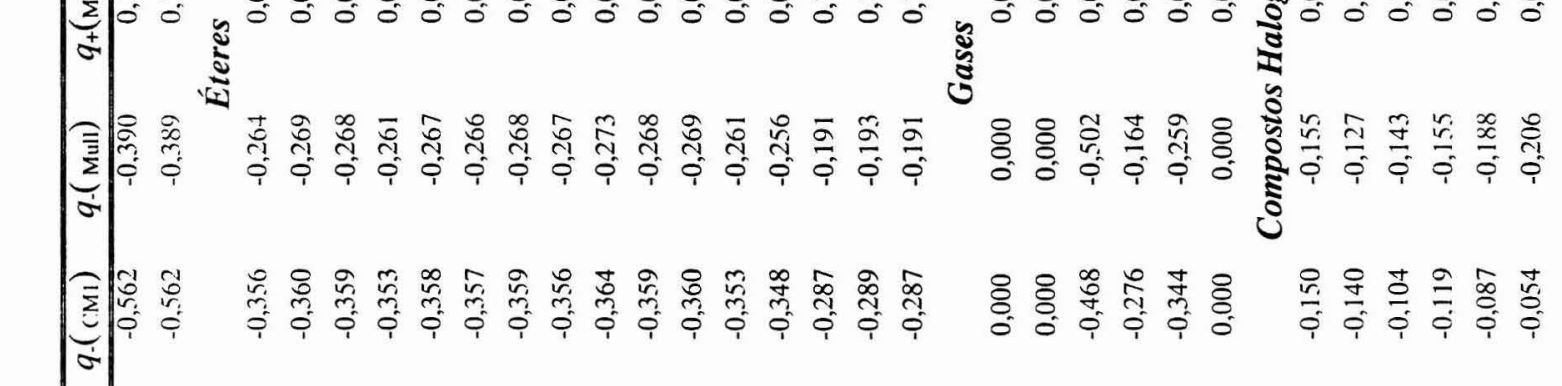




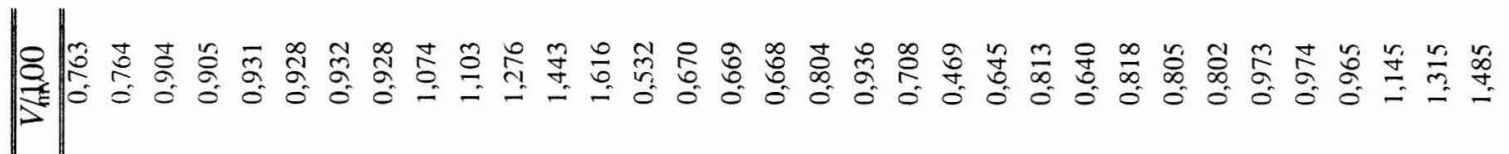

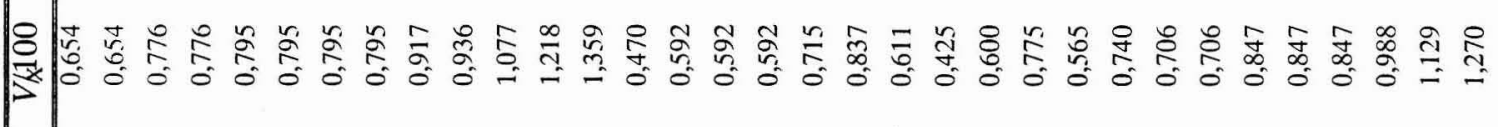

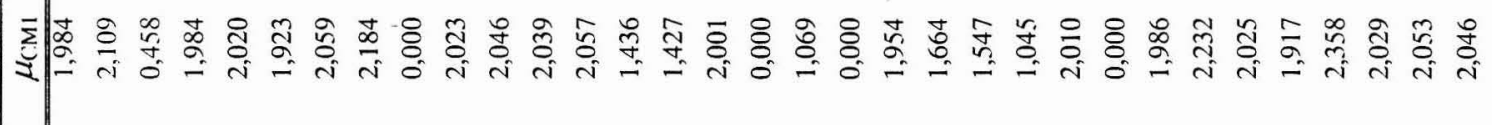

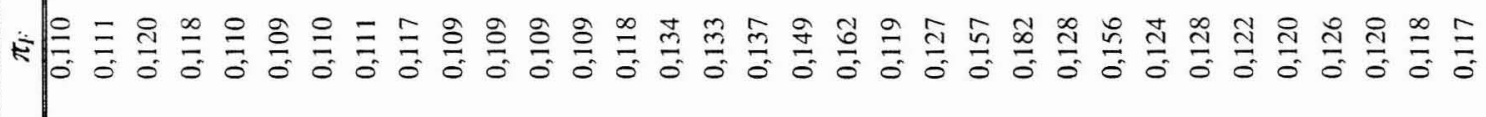

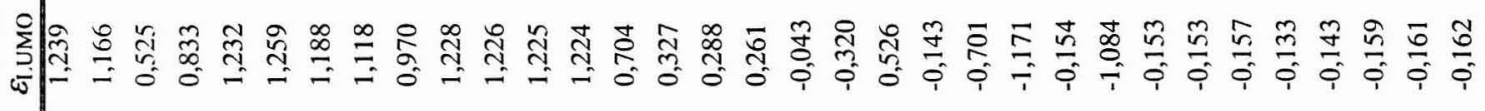

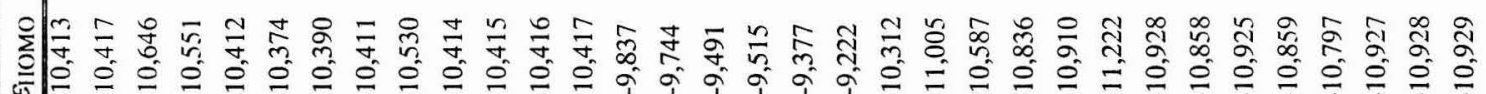

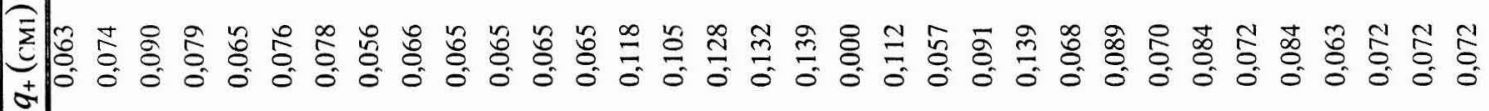

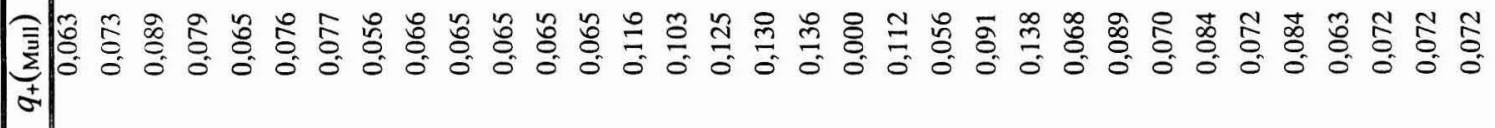

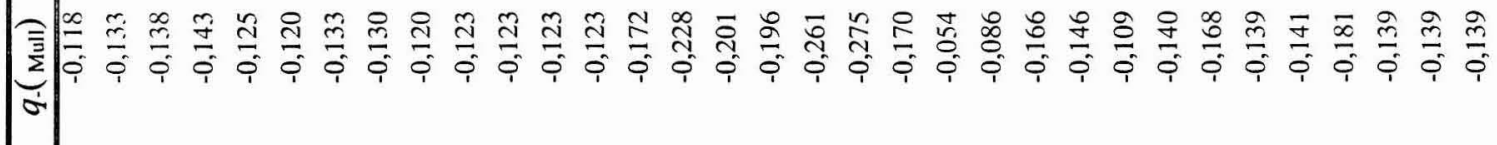

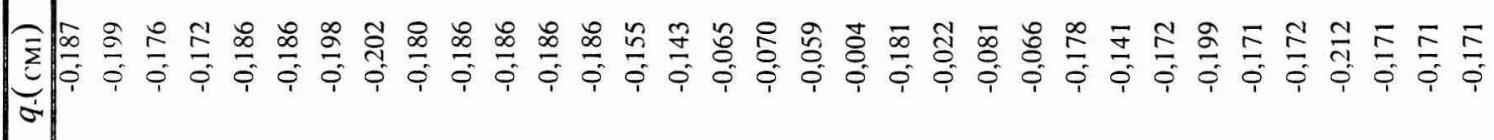

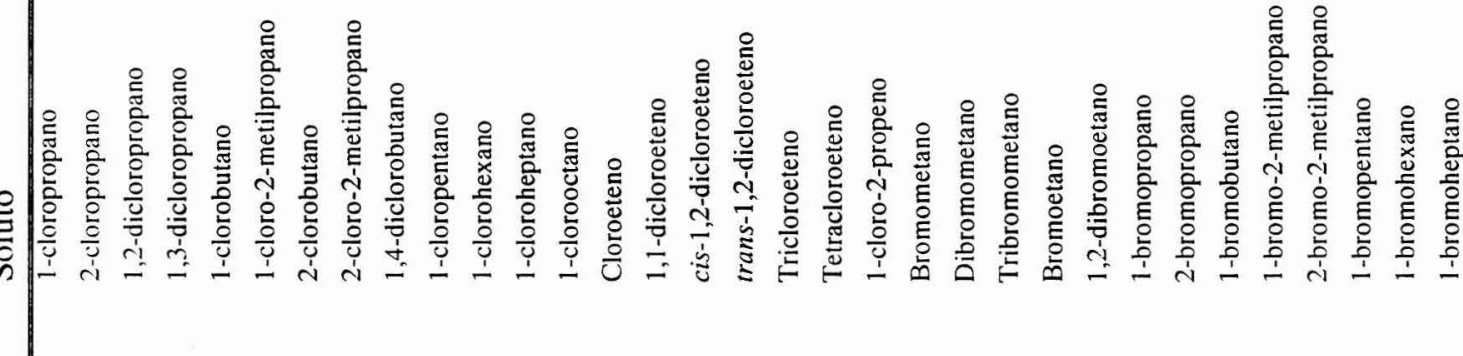

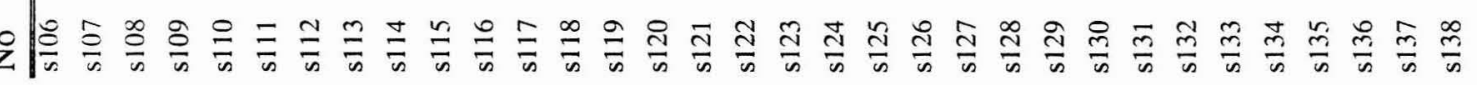




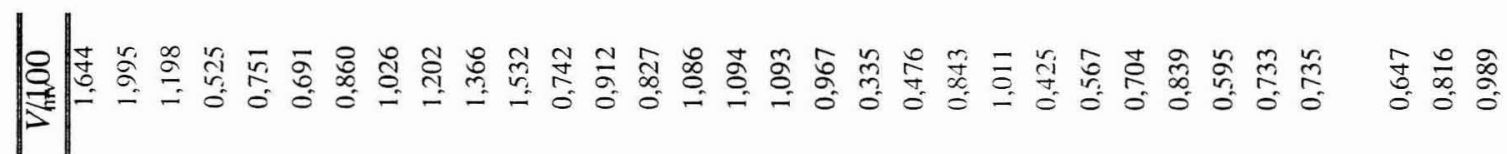

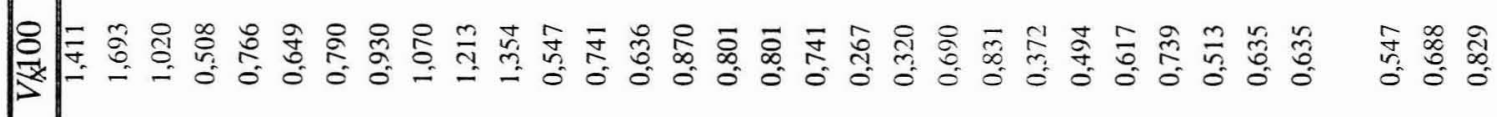

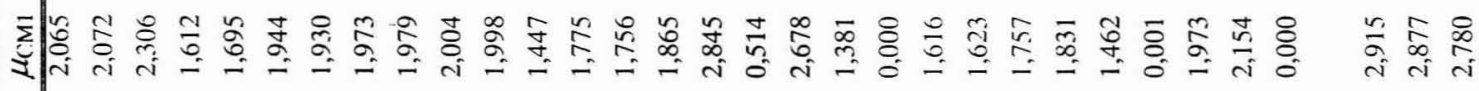

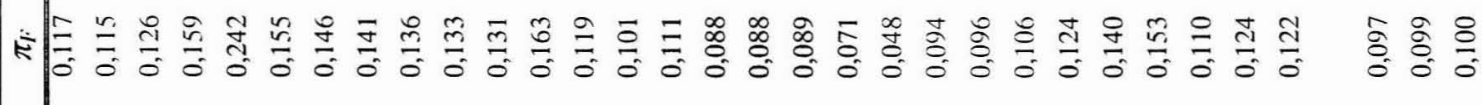

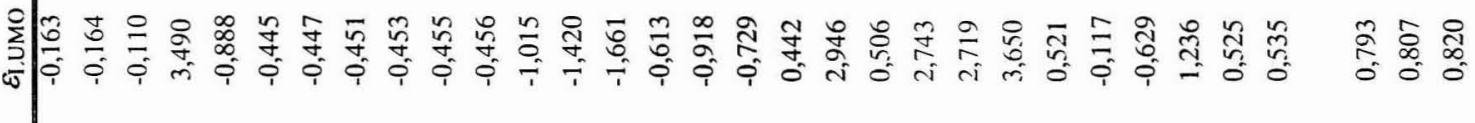

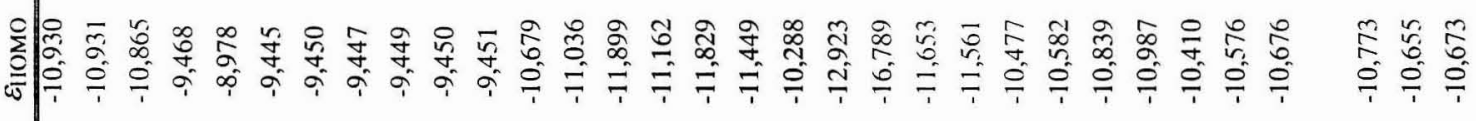

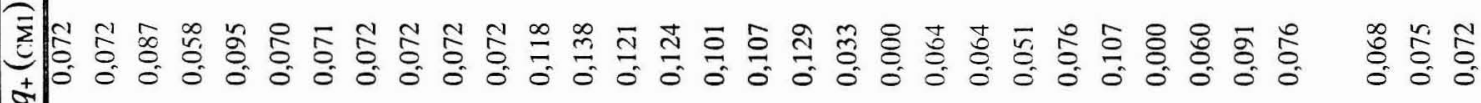

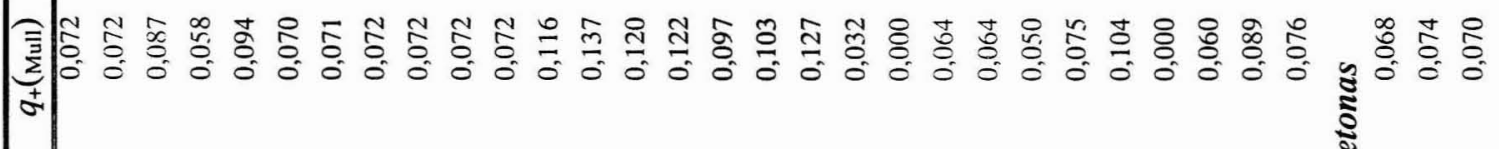

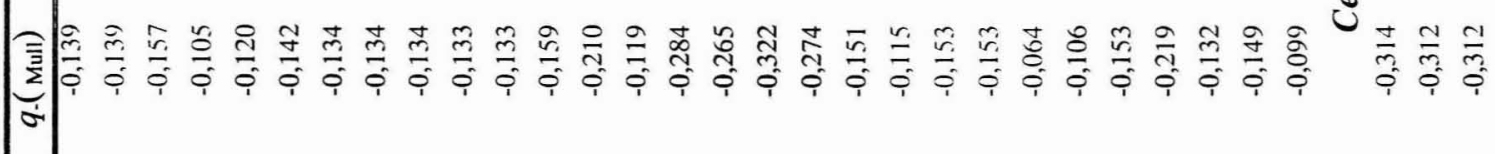

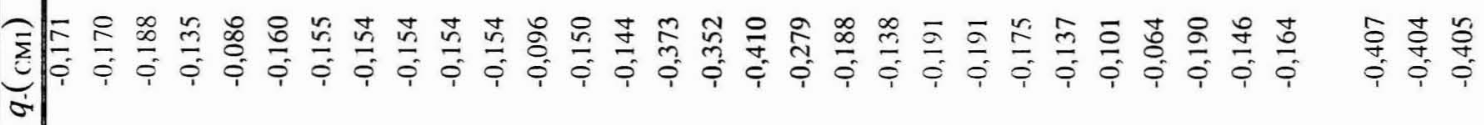




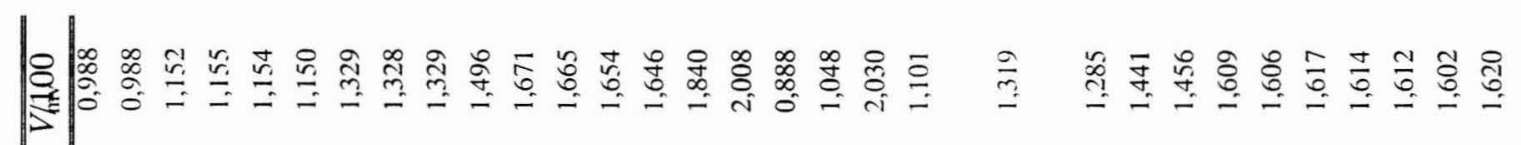

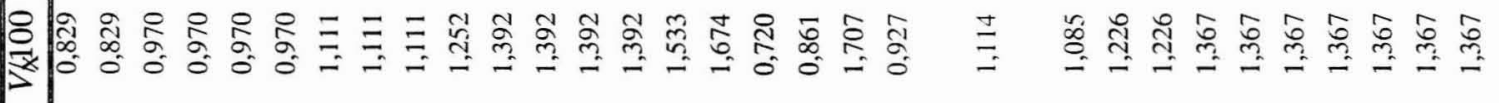

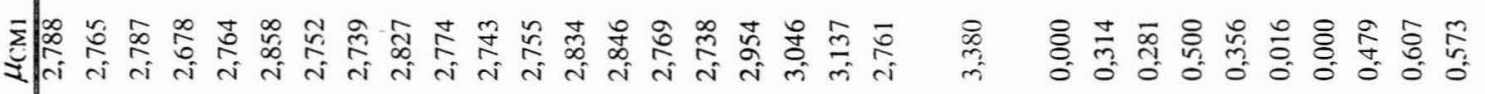

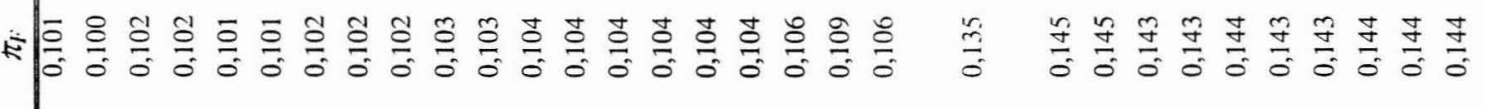

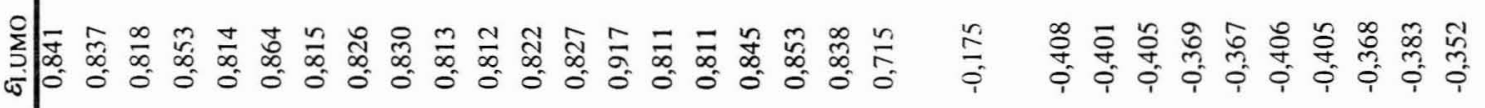

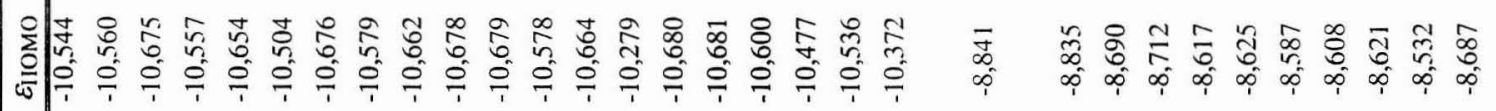

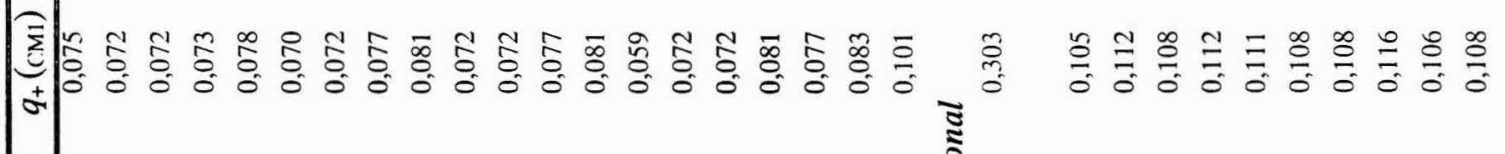

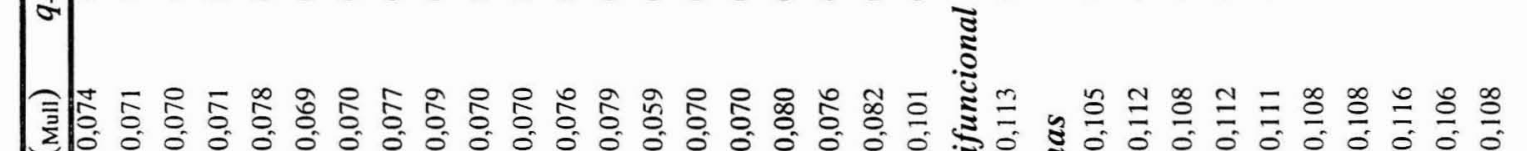

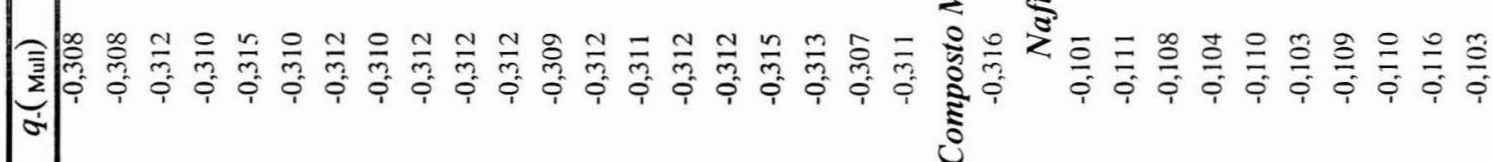

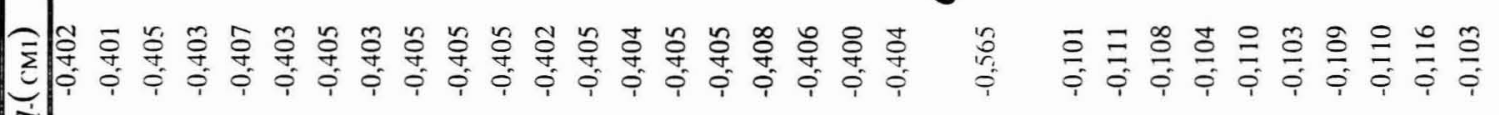




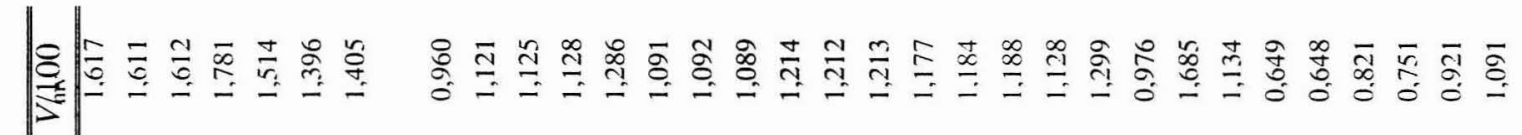

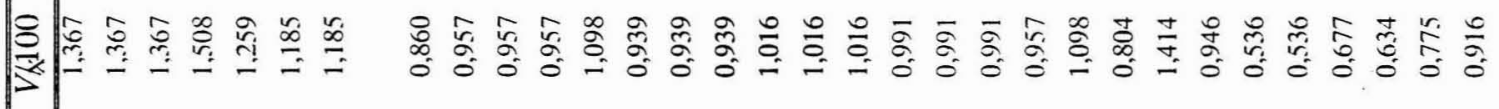

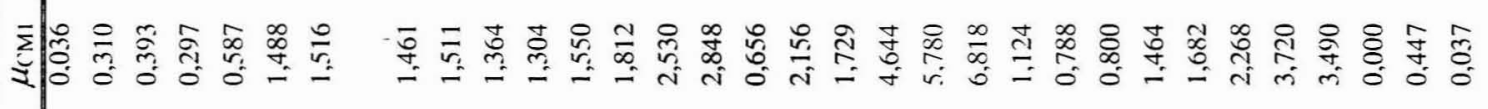

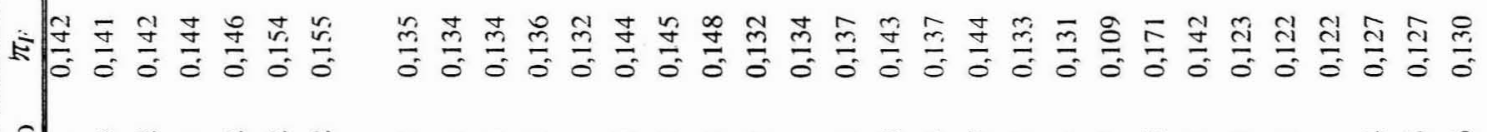

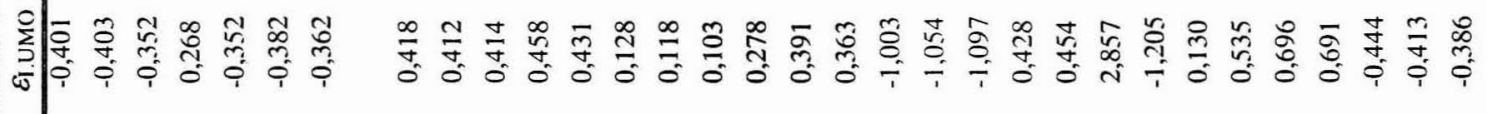

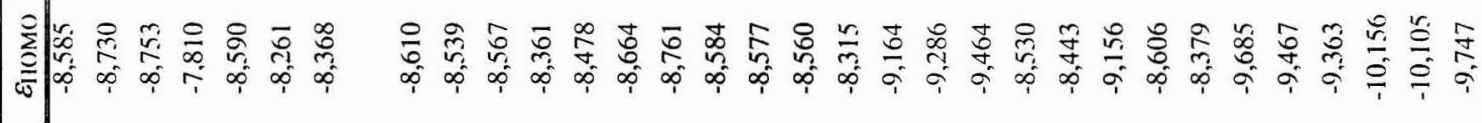

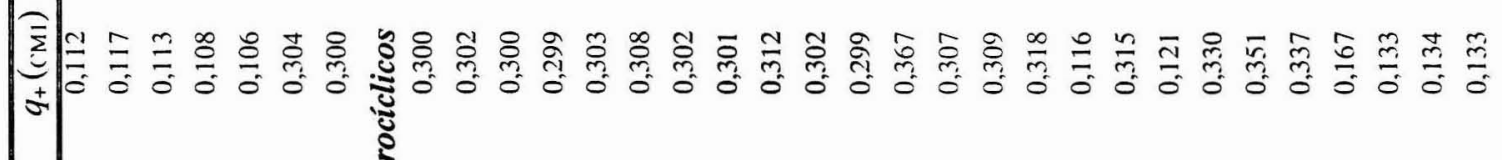

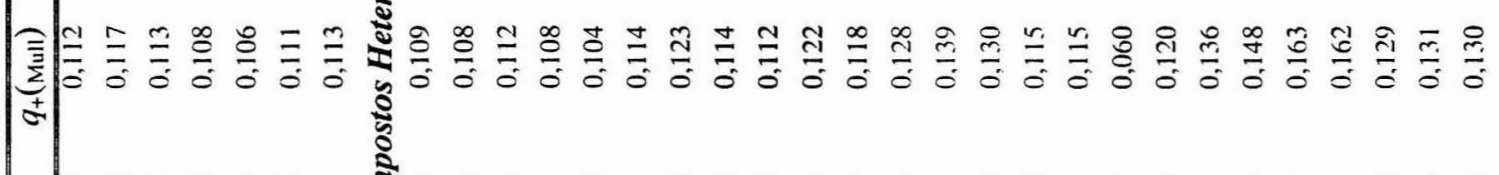

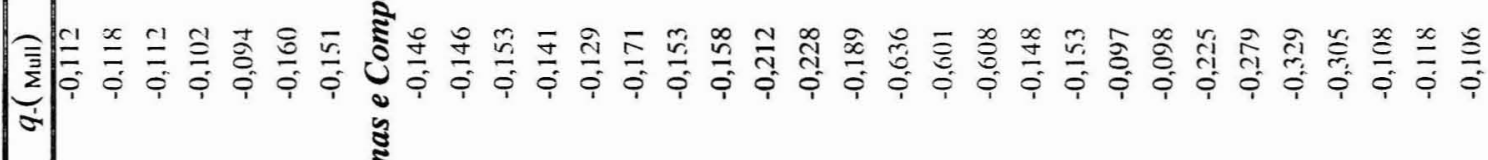

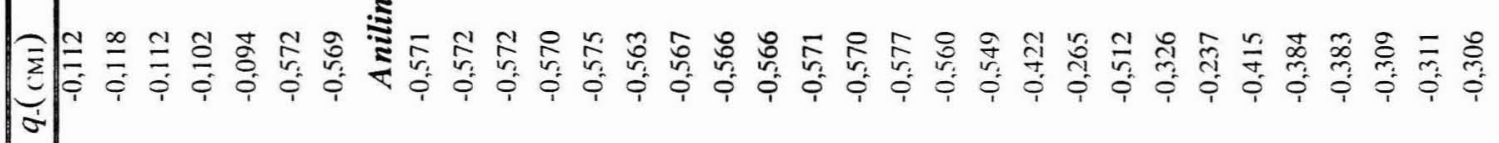




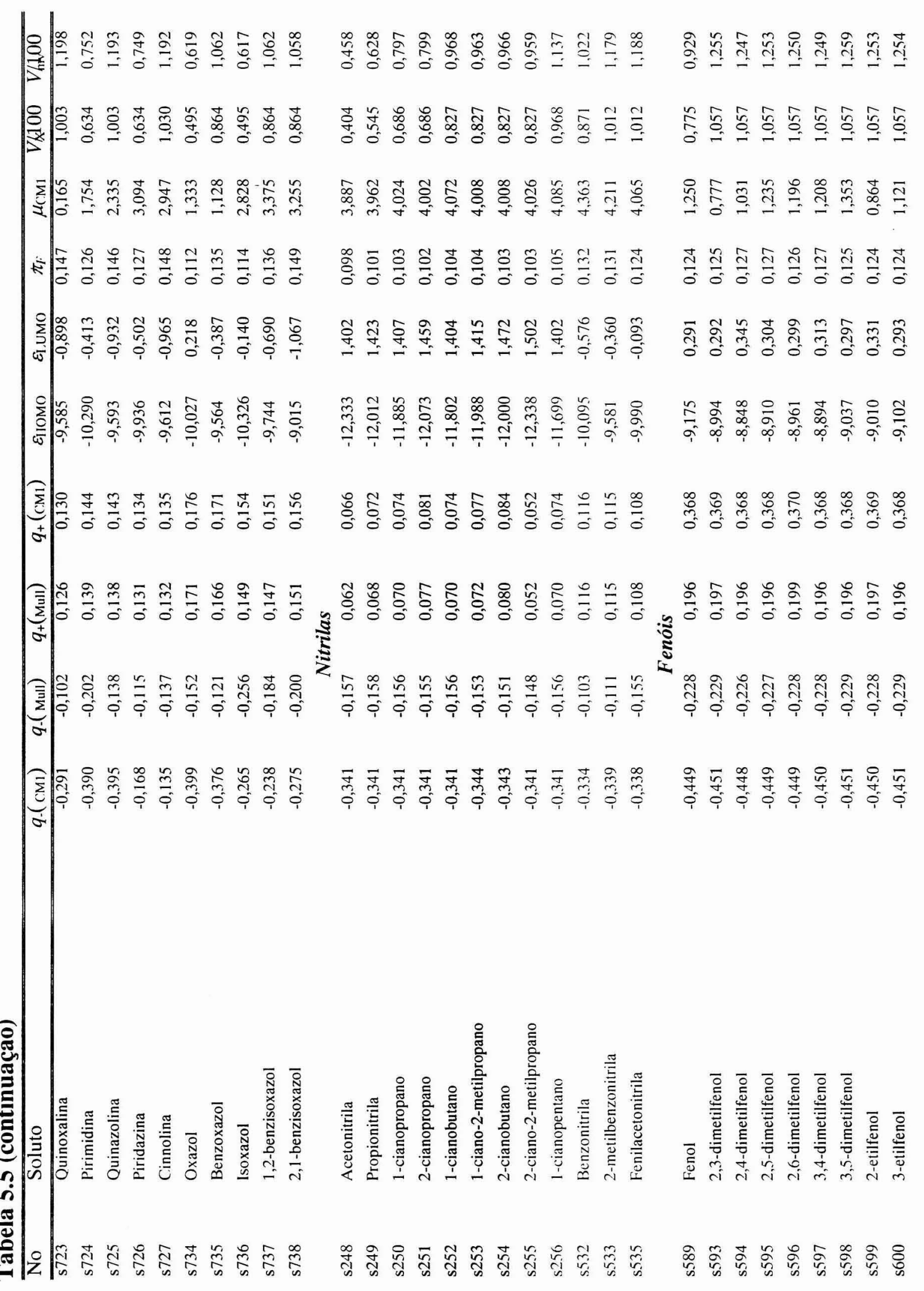




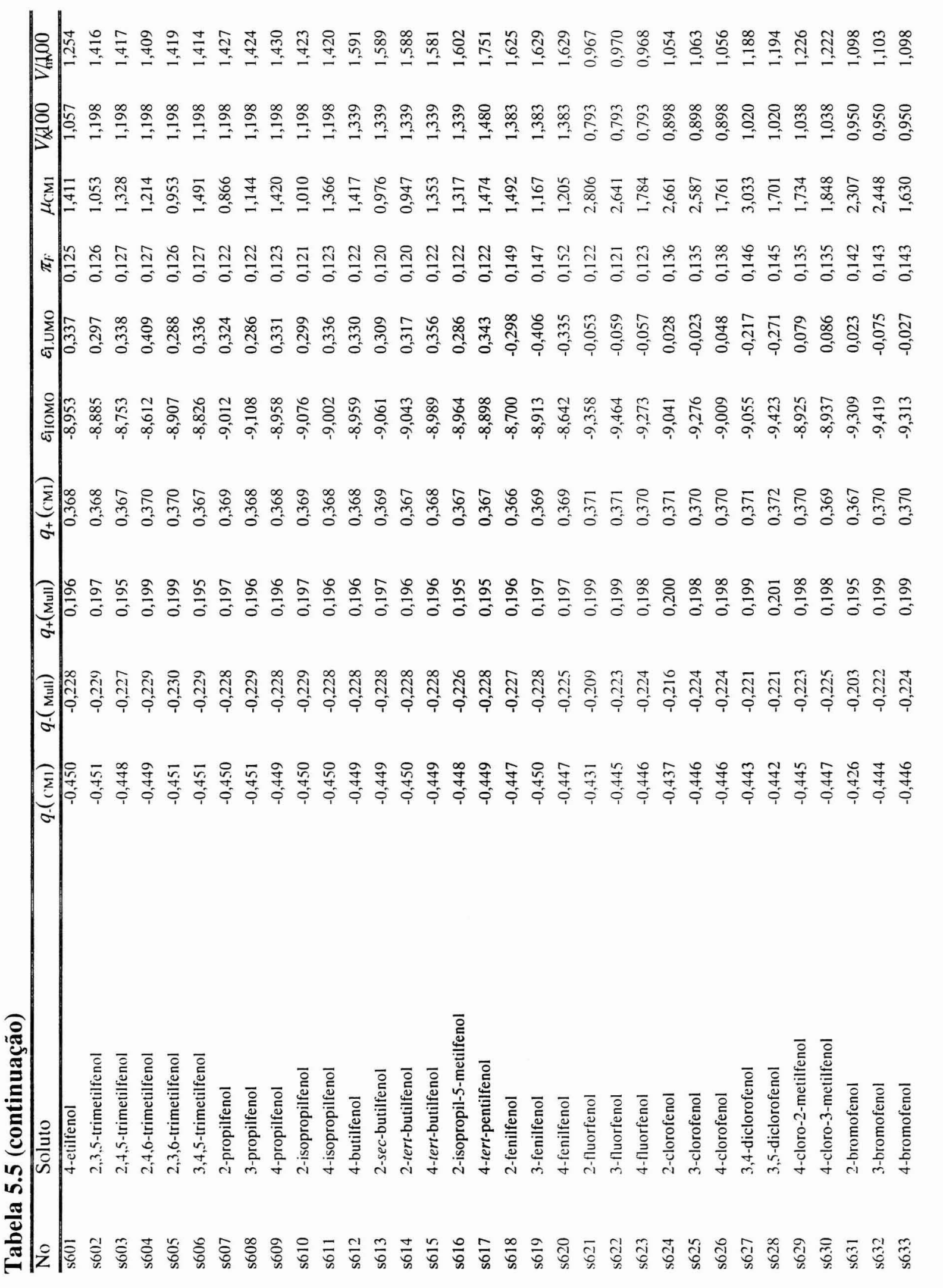




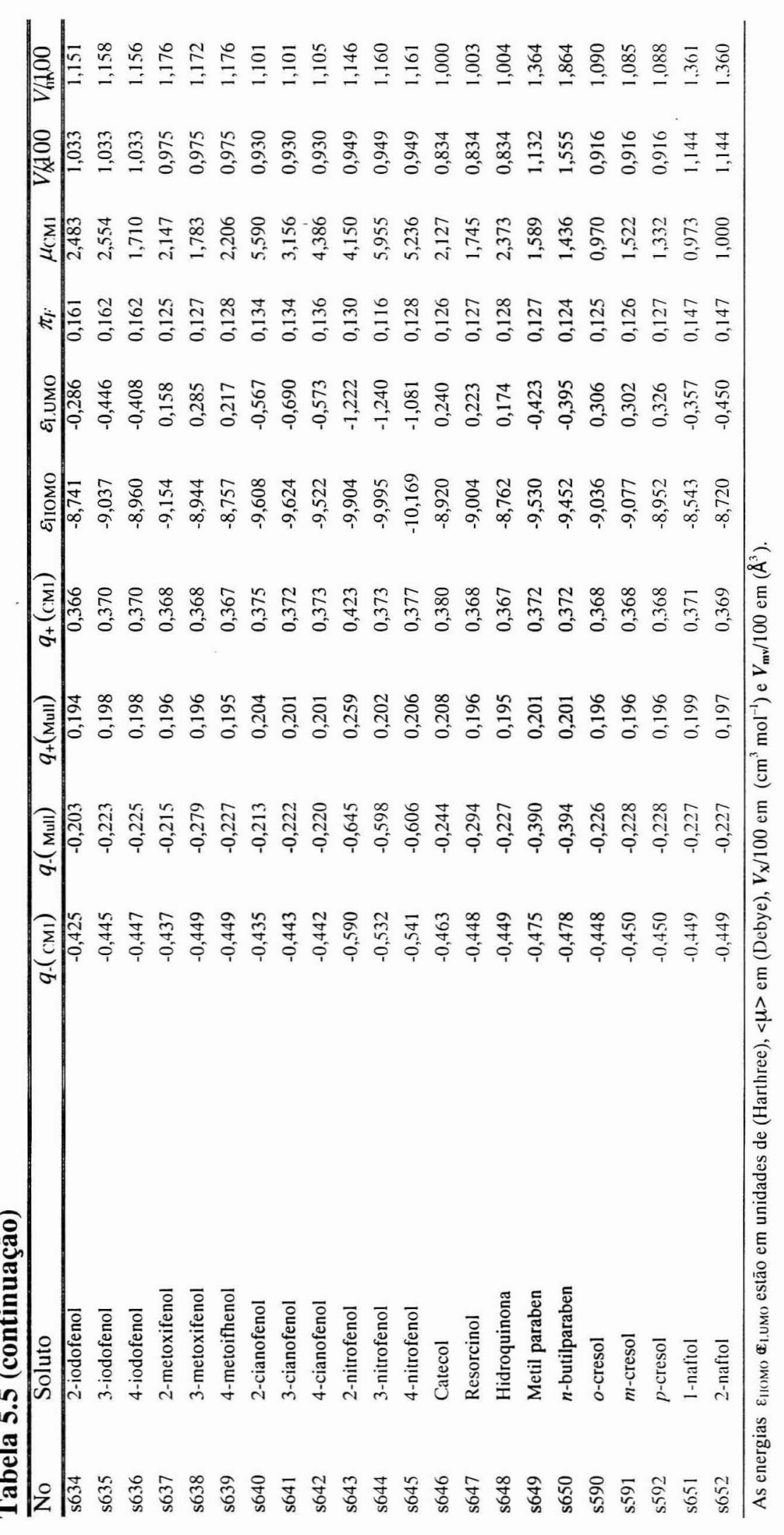


Todos os sete parâmetros teóricos foram calculados para geometria otimizada pelo algoritmo BFGS (Broyden-Flechter-Goldfarb-Shanno) ${ }^{\mathbf{1 9 - 2 2}}$ como implementado nos programas $A M S O L-5.4$ e $M O P A C-93$. A geometria molecular é considerada otimizada quando passa pelos critérios de convergência adotado. Neste estudo, todas as moléculas foram otimizadas, calculando-se a força resultante sobre os átomos na molécula, através do deslocamento dos átomos na direção determinada pela força até que a energia total convirja para um valor mínimo pre definido e que não haja diferença de energia entre dois ciclos $S C F$ consecutivos, ou seja, quando atingimos um ponto de mínima na superfície de energia potencial. O critério adotado neste estudo para a convergência do processo $S C F$ foi de $10^{-8}$ $\mathrm{kcal} \mathrm{mol}^{-1}$ para a diferença de energia entre dois ciclos $S C F$ e $\leq 0,01$ para a norma do gradiente

Análogo ao modelo de Famini, podemos descrever o logaritmo da constante de incorporação (propriedade físico-química de interesse) através de uma relação linear teórica de energia livre de solvatação dada pela equação (5.25) a qual contém sete parâmetros teóricos.

$\log \left(K_{s}\right)=\mathrm{a}_{0}+\mathrm{a}_{1} \varepsilon_{H O M O}+\mathrm{a}_{2} \varepsilon_{\text {LUMO }}+\mathrm{a}_{3}<\mu>+\mathrm{a}_{4} \pi_{\mathrm{F}}+\mathrm{a}_{5} q_{+}+\mathrm{a}_{6}\left|q_{-}\right|+\mathrm{a}_{7}(\mathrm{~V} / 100)$

Cabe aqui observar que neste estudo mostraremos pela primeira vez uma análise detalhada do uso de regressão linear múltipla usando parâmetros teóricos (obtidos através de cálculos de química quântica) como descritores para estudar solubilização em sistema micelar. No próximo capítulo mostraremos os resultados do uso destes parâmetros teóricos como descritores em estudos que envolvam transferência de um soluto e sua cavidade da fase aquosa até a micela micelizada.

\section{Referências}

1. Hopfinger, A.J.; 1980, J. Am. Chem. Soc.;102,7126.

2. McGowan, J.C.; 1978, J. App. Chem. Biotechnol.;28,599.

3. McGowan, J.C.; Ahmad, P.; Mellors, A.; 1979, Canad. J. Pharm. Sci.;14,72.

4. McGowan, J.C.; 1984, J. App. Chem. Biotechnol.;34A,38.

5. Storer, J.W.; Giensen,D.J.; Cramer, J.C.; Truhlar, D.G.; 1995, J Comput-Aided Mol. Design; $9,87$.

6. Mulliken, R.S.; 1935, J. Chem. Phys., 3, 564.

7. Mulliken, R.S.; 1955, J. Chem. Phys., 23, 1833.

8. Mulliken, R.S.; 1962, J. Chem. Phys., 36, 3428.

9. Armstrong, D.R.; Perkins, P.G.; Stewart, J.J.P.; 1973, J. Chem. Soc. Dalton Trans.;838.

10. Jorgensen, W.L.; Chandrasekhar, J.; Madura, J.D.; Impey, R.W.; Klein, 1983, J. Chem. Phy.; 79,926.

11. Scrocco, E.; 1978, Adv. Quantum Chem.,11,115.

12. Srebrenik, S.; Weinstein, H.; Pauncz, R.; 1972, Chem. Phys. Lett.,20,4,19.

13. Stark,B.; em Hellwege,K.H. e Hellwege, A.M. (Eds),1982, Molecualr Constants from Microwave Spectroscopy, Landolt-Börnstein, New Series, Group II, Vol 4, SpringerVerlang, Berlim,136. 
14. Demaison, J.; Hutner, W.; Stark, B.; Buck, I.; Ticher, R.;Winnewisser, M.; em Hellwege, K.H.; (Eds), 1974, Molecualr Constants, Landolt-Börnstein, New Series, Group II, Vol. 6, Springer-Verlang, Berlim,261.

15. Demaison, J.; Hutner, W.; Tiemann, E.; em Hellwege, K.H.; (Eds), 1982, Molecular Constants, Landolt-Börnstein, New Series, Group II, Vol. I ta, Springer-Verlang, Berlim, 584 .

16. Nelson, R.D.; Lide, D.R.; Maryott, A. A.; 1967, Natl. Stand., Ref. Data Ser., United States National Bureau of Standards, NSRDS-NBS 10.

17. Kurtz, H.A.; Stewart, J.J.P.; Dieter, J.; 1990, J. Comp. Chem.;82,11.

18. Dewar, M.J.S.; Stewart, J.J.P. ; 1994, Chem. Phys.Lett.;111,416.

19. Broyden, C.G.;1967, Math. Comput., 21, 368.

20. Fletcher, R.;1970, J. Comput.,13, 317.

21. Goldfarb, D.; 1970, Math. Comput., 24,23.

22. Shanno, F.; 1970, Math. Comput.,24, 647. 


\section{Capítulo 6}

Nossos resultados mostram que as equações de regressão linear teórica usadas para descrever a incorporação de solutos não iônicos em micelas formadas por detergentes aniônicos são quimicamente consistentes com aquelas obtidas empregando os parâmetros empíricos de Abraham. Porém a metodologia apresenta algumas limitações quando aplicada a micelas formadas por detergentes catiônicos e não iônicos. 


\subsection{Resultados}

Neste capítulo, mostraremos pela primeira vez uma análise detalhada do uso de parâmetros teóricos como descritores, para estudar incorporação de solutos não iônicos em micelas formadas por detergentes. Discutiremos os resultados, para a incorporação de uma série de solutos em micelas aquosas formadas por $S D S, S D S$ modificado pela adição do polímero poli(etilenoglicol) (PEG-poly (ethylene glycol)), brometo de dodecíltrimetilamonio (DTAB - dodecyltrimethylammonium bromide), brometo de hexadeciltrimetilamonio, (CTAB - hexadecyltrimethylammonium bromide) e éter dodecíl (23) polioxietileno (Brij-35 - polyoxyethylene (23) dodecyl ether).

\subsubsection{Micelas formadas por SDS e SDS-PEG}

Primeiro, mostraremos os resultados da análise por regressão linear múltipla, efetuados com a equação (5.25), para parâmetros calculados através do hamiltoniano MNDO. A incorporação de 66 solutos não iônicos em micelas aquosas formadas por $S D S$, é descrita pela seguinte equação:

$$
\begin{gathered}
\log \left(K_{s}\right)=-0,48-3,76\left|q_{-}\right|+1,27 q_{+}+2,97\left(\mathrm{~V}_{x} / 100\right) \\
(S D S: n=66 ; R=0,969 ; s d=0,21 ; F=316)
\end{gathered}
$$

Os coeficientes dos parâmetros teóricos $\varepsilon_{\mathrm{HOMO}}, \varepsilon_{\mathrm{LUMO}},<\mu>$ e $\pi_{\mathrm{F}}$ da equação (5.25) são essencialmente zero e não são estatisticamente significativos; por isso, não aparecem na equação (6.1). O relativo sucesso do emprego da metodologia no estudo de incorporação de solutos não iônicos em micelas de $\operatorname{SDS}^{\mathbf{1}}$ (os resultados são apresentados com maiores detalhes no Apêndice A) levou-nos a aplicar a metodologia para o mesmo conjunto de solutos porém usando parâmetros calculados a partir do hamiltoniano $M N D O-P M 3$, o qual difere do hamiltoniano $M N D O$ no método de parametrização, no conjunto de parâmetros usados para reproduzir o calor de formação de moléculas e no segundo termo da equação (4.59) acrescentado para corrigir os excessos de longo alcance no termo de repulsão caroçocaroço.

Na Tabela 6.1, mostramos os valores de log $\left(K_{s}\right)$ obtidos por técnicas experimentais e os valores dos parâmetros teóricos calculados a partir do hamiltoniano MNDO-PM3. Cabe aqui observar que, no caso do gás nobre Argônio, somente calculamos o parâmetro $V_{\mathrm{x}}$; consequentemente, este soluto só entra no cômputo da equação de regressão final. A equação de regressão final que correlaciona melhor os parâmetros teóricos com $\log \left(K_{s}\right)$ contém somente os termos $|q-|, q_{+}$e $\mathrm{V}_{\mathrm{x}} / 100$.

$$
\begin{gathered}
\log \left(K_{s}\right)=-0,56-2,94|q-|+1,66 q_{+}+3,00\left(\mathrm{~V}_{\mathrm{x}} / 100\right) \\
(S D S: n=66 ; R=0,971 ; s d=0,20 ; F=345,3)
\end{gathered}
$$


Tabela 6.1 Valores de log $\left(K_{s}\right)$ e dos sete parâmetros teóricos (calculados através do hamiltoniano $M N D O-P M 3$ ) usados para descrever a incorporação de (66) solutos não iônicos em micelas aquosas formadas por $S D S$.

\begin{tabular}{|c|c|c|c|c|c|c|c|c|c|}
\hline No & Soluto & ${ }^{a} \log \left(K_{s}\right)$ & $|q|$. & $q_{+}$ & $\pi_{\mathrm{F}}$ & $\varepsilon_{\text {HOMO }}$ & $\varepsilon_{\text {LUMO }}$ & $\mu_{\mathrm{CM} 1}$ & $\mathrm{~V}_{\mathrm{x}} / 100$ \\
\hline \multicolumn{10}{|c|}{ Álcoois } \\
\hline s324 & Metanol & $-0,574$ & 0,528 & 0,355 & 0,085 & $-11,138$ & 3,508 & 1,593 & 0,308 \\
\hline s325 & Etanol & $-0,125$ & 0,529 & 0,356 & 0,092 & $-11,126$ & 3,358 & 1,607 & 0,449 \\
\hline s326 & 1-Propanol & 0,308 & 0,530 & 0,359 & 0,096 & $-10,883$ & 3,229 & 1,516 & 0,590 \\
\hline s327 & 2-Propanol & 0,146 & 0,530 & 0,359 & 0,096 & $-11,038$ & 3,276 & 1,583 & 0,590 \\
\hline $\mathrm{s} 328$ & 1-Butanol & 0,724 & 0,530 & 0,359 & 0,098 & $-10,887$ & 3,159 & 1,531 & 0,731 \\
\hline s330 & 2-Butanol & 0,477 & 0,534 & 0,363 & 0,098 & $-10,920$ & 3,186 & 1,712 & 0,731 \\
\hline s331 & 2-Metil-2-Propanol & 0,663 & 0,525 & 0,359 & 0,098 & $-11,278$ & 3,254 & 1,553 & 0,731 \\
\hline $\mathrm{s} 332$ & 1-Pentanol & 1,224 & 0,530 & 0,359 & 0,100 & $-10,889$ & 3,114 & 1,497 & 0,872 \\
\hline s336 & 3-Metil-1-Butanol & 1,161 & 0,528 & 0,358 & 0,100 & $-10,782$ & 3,155 & 1,570 & 0,872 \\
\hline s340 & 1-Hexanol & 1,624 & 0,530 & 0,359 & 0,101 & $-10,890$ & 3,083 & 1,522 & 1,013 \\
\hline s341 & 2-Hexanol & 1,591 & 0,534 & 0,363 & 0,101 & $-10,936$ & 3,050 & 1,764 & 1,013 \\
\hline s347 & 1-Heptanol & 2,071 & 0,530 & 0,359 & 0,102 & $-10,891$ & 3,061 & 1,491 & 1,154 \\
\hline s351 & 1-Octanol & 2,437 & 0,530 & 0,359 & 0,103 & $-10,893$ & 3,046 & 1,518 & 1,295 \\
\hline s653 & Alcool Benzílico & 0,996 & 0,522 & 0,357 & 0,120 & $-9,641$ & 0,319 & 1,618 & 0,916 \\
\hline \multicolumn{10}{|c|}{ Gases e Hidrocarbonetos Alifáticos } \\
\hline s010 & Oxigênio & $-0,148$ & 0,000 & 0,000 & 0,053 & $-10,733$ & $-0,982$ & 0,000 & 0,183 \\
\hline s009 & Argônio & $-0,137$ & 0,000 & 0,000 & & & & & 0,190 \\
\hline s015 & Metano & 0,079 & 0,000 & 0.000 & 0,084 & $-13,642$ & 4,245 & 0,000 & 0,250 \\
\hline s016 & Etano & 0,792 & 0,000 & 0,000 & 0,094 & $-11,976$ & 3,892 & 0,000 & 0,390 \\
\hline s017 & Propano & 1,342 & 0,000 & 0,000 & 0,097 & $-11,506$ & 3,715 & 0,000 & 0,531 \\
\hline s049 & Ciclohexano & 2,431 & 0,000 & 0,000 & 0,107 & $-11,290$ & 3,476 & 0,000 & 0,845 \\
\hline \multicolumn{10}{|c|}{ Hidrocarbonetos Aromáticos e Derivados } \\
\hline s389 & Benzeno & 1,354 & 0,102 & 0,102 & 0,122 & $-9,751$ & 0,396 & 0,000 & 0,716 \\
\hline s564 & Nitrobenzeno & 1,356 & 0,534 & 0,134 & 0,124 & $-10,602$ & $-1,134$ & 5,075 & 0,891 \\
\hline$s 465$ & Clorobenzeno & 1,841 & 0,102 & 0,117 & 0,133 & $-9,388$ & 0,063 & 1,583 & 0,839 \\
\hline s390 & Tolueno & 1,733 & 0,109 & 0,105 & 0.125 & $-9,442$ & 0,377 & 0,255 & 0,857 \\
\hline s391 & Etilbenzeno & 2,438 & 0,115 & 0,112 & 0,122 & $-9,444$ & 0,388 & 0,300 & 0,998 \\
\hline s394 & $p$-Xileno & 2.209 & 0.101 & 0,105 & 0,125 & $-9,182$ & 0,357 & 0.025 & 0,998 \\
\hline$s 490$ & Anisole & 1,462 & 0,287 & 0,120 & 0,126 & $-9,140$ & 0,338 & 1,080 & 0.916 \\
\hline$s 423$ & Nattaleno & 2,566 & 0,101 & 0,105 & 0,145 & -8.835 & -0.408 & 0,000 & 1.085 \\
\hline$s 424$ & 1-Metílnaftaleno & 3,064 & 0,111 & 0,112 & 0,145 & $-8,690$ & $-0,401$ & 0,314 & 1,226 \\
\hline s421 & Bifenil & 3,096 & 0,102 & 0,111 & 0,146 & -8.918 & $-0,361$ & 0,000 & 1,324 \\
\hline s443 & Antraceno & 3,772 & 0,100 & 0,108 & 0,171 & $-8,248$ & $-0,970$ & 0,000 & 1,454 \\
\hline \multicolumn{10}{|c|}{ Ácidos Carboxilicos e Derivados } \\
\hline s576 & Acetanilida & 1,255 & 0,431 & 0,332 & 0,130 & $-9,028$ & 0,155 & 3,426 & 1,113 \\
\hline s250 & 1-Cianopropano & 0,633 & 0,341 & 0,074 & 0,103 & $-11,885$ & 1,407 & 4,024 & 0,686 \\
\hline s580 & Ácido Benzóico & 1,560 & 0,519 & 0,397 & 0,122 & $-10,133$ & $-0,533$ & 2,386 & 0,932 \\
\hline s569 & Benzamida & 0,882 & 0,645 & 0,331 & 0,128 & $-10,040$ & $-0,410$ & 3,649 & 0,973 \\
\hline $\mathrm{s} 532$ & Benzonitrila & 1,387 & 0,334 & 0,116 & 0,132 & $-10,095$ & $-0,576$ & 4,363 & 0,871 \\
\hline s582 & 3-Metil Ácido Benzóico & 1,919 & 0,519 & 0,396 & 0,123 & $-9,817$ & $-(0,496$ & 2,663 & 1,073 \\
\hline s583 & 4- Metil Ácido Benzóico & 1,851 & 0,520 & 0,396 & 0,125 & $-9,865$ & $-0,522$ & 2,724 & 1,073 \\
\hline \multicolumn{10}{|c|}{ Éteres } \\
\hline s169 & 1,4-Dioxano & 0,114 & 0,348 & 0,064 & 0,103 & $-10,448$ & 2,840 & 0,000 & 0,681 \\
\hline
\end{tabular}


Tabela 6.1 (continuação)

\begin{tabular}{|c|c|c|c|c|c|c|c|c|c|}
\hline No & Soluto & ${ }^{a} \log \left(K_{\mathrm{s}}\right)$ & $|q-|$ & $q_{+}$ & $\pi_{\mathrm{F}}$ & $\varepsilon_{\text {НОмо }}$ & $\varepsilon_{\text {LUMO }}$ & $\mu_{\mathrm{CM} 1}$ & $\mathrm{~V}_{\mathrm{x}} / 100$ \\
\hline \multicolumn{10}{|c|}{ Compostos Halogenados } \\
\hline s144 & Iodoetano & 1,342 & 0,160 & 0,070 & 0,155 & $-9,445$ & $-0,445$ & 1,944 & 0,649 \\
\hline s146 & 1-Iodobutano & 2,057 & 0,154 & 0,072 & 0,141 & $-9,447$ & $-0,451$ & 1,973 & 0,930 \\
\hline s151 & Halotano & 1,057 & 0,150 & 0,138 & 0,119 & $-11,036$ & $-1,420$ & 1,775 & 0,741 \\
\hline s094 & Diclorometano & 0,556 & 0,137 & 0,076 & 0,124 & $-10,582$ & 0,521 & 1,831 & 0,494 \\
\hline s095 & Cloroformio & 1,025 & 0,101 & 0,107 & 0,140 & $-10,839$ & $-0,117$ & 1,462 & 0,617 \\
\hline s096 & Tetraclorometano & 1,548 & 0,064 & 0,000 & 0,153 & $-10,987$ & $-0,629$ & 0,000 & 0,739 \\
\hline \multicolumn{10}{|c|}{ Aldeídos e Cetonas } \\
\hline s181 & Propanona & $-0,045$ & 0,407 & 0,068 & 0,097 & $-10,773$ & 0,793 & 2,915 & 0,547 \\
\hline s182 & 2-Butanona & 0,327 & 0,404 & 0,075 & 0,099 & $-10,655$ & 0,807 & 2,877 & 0,688 \\
\hline s186 & 2-Hexanona & 1,235 & 0,405 & 0,072 & 0,102 & $-10,675$ & 0,818 & 2,787 & 0,970 \\
\hline s190 & 2-Heptanona & 1,676 & 0,405 & 0,072 & 0,102 & $-10,676$ & 0,815 & 2,752 & 1,111 \\
\hline s506 & Acetofenona & 1,522 & 0,408 & 0,119 & 0,123 & $-10,008$ & $-0,355$ & 2,989 & 1,014 \\
\hline s183 & 2-Pentanona & 0,799 & 0,405 & 0,072 & 0,100 & $-10,673$ & 0,820 & 2,780 & 0,829 \\
\hline s512 & Etilfenil Cetona & 1,732 & 0,404 & 0,118 & 0,122 & $-9,999$ & $-0,318$ & 2,890 & 1,155 \\
\hline s513 & Benzofenona & 2,653 & 0,389 & 0,111 & 0,132 & $-9,888$ & $-0,275$ & 3,007 & 1,481 \\
\hline \multicolumn{10}{|c|}{ Aminas Aromáticas } \\
\hline s536 & Anilina & 0,676 & 0,571 & 0,300 & 0,135 & $-8,610$ & 0,418 & 1,461 & 0,816 \\
\hline s539 & $p$-Toluidina & 1,278 & 0,570 & 0,299 & 0,136 & $-8,361$ & 0,458 & 1,304 & 0,957 \\
\hline \multicolumn{10}{|c|}{ Fenóis e Naftóis } \\
\hline s589 & Fenol & 0,967 & 0,449 & 0,368 & 0,124 & $-9,175$ & 0,291 & 1,250 & 0,775 \\
\hline s623 & 4-Fluorofenol & 1,204 & 0,446 & 0,370 & 0,123 & $-9,273$ & $-0,057$ & 1,784 & 0,793 \\
\hline s626 & 4-Clorofenol & 1,583 & 0,446 & 0,370 & 0,138 & $-9,009$ & 0,048 & 1,761 & 0,898 \\
\hline s633 & 4-Bromofenol & 1,826 & 0,446 & 0,370 & 0,143 & $-9,313$ & $-0,027$ & 1,630 & 0,950 \\
\hline s636 & 4-Iodofenol & 2,089 & 0,447 & 0,370 & 0,162 & $-8,960$ & $-0,408$ & 1,710 & 1,033 \\
\hline s645 & 4-Nitrofenol & 1,284 & 0,541 & 0,377 & 0,128 & $-10,169$ & $-1,081$ & 5,236 & 0,949 \\
\hline s590 & $o$-Cresol & 1,330 & 0,448 & 0,368 & 0,125 & $-9,036$ & 0,306 & 0,970 & 0,916 \\
\hline s591 & $m$-Cresol & 1,348 & 0,450 & 0,368 & 0,126 & $-9,077$ & 0,302 & 1,522 & 0,916 \\
\hline s592 & $p$-Cresol & 1,372 & 0,450 & 0,368 & 0,127 & $-8,952$ & 0,326 & 1,332 & 0,916 \\
\hline$s 651$ & 1-Naftol & 2,017 & 0,449 & 0,371 & 0,147 & $-8,543$ & $-0,357$ & 0,973 & 1,144 \\
\hline$s 652$ & 2-Naftol & 2,232 & 0,449 & 0,369 & 0,147 & $-8,720$ & $-0,450$ & 1,000 & 1,144 \\
\hline
\end{tabular}

${ }^{2}$ Os valores de $\log \left(K_{s}\right)$ foram obtidos a partir da média dos valores experimentais de $\left(K_{s}\right)$ apresentados na referência ${ }^{2}$, as energias de $\varepsilon_{\text {номо }}$ e $\varepsilon_{\text {Luмо }}$ são expressas em (hartree), momento de dipolo $<\mu>\mathrm{em}$ Debye $(D)$ e $\mathrm{V}_{\mathrm{x}} \mathrm{em}\left(\mathrm{cm}^{3} \mathrm{~mol}^{-1}\right)$.

Para que os parâmetros teóricos escolhidos espelhem as propriedades específicas no ambiente de solubilização dos solutos, os valores e os sinais dos coeficientes da equação de regressão linear teórica, devem ser compatíveis com os da equação (2.7). Esta foi obtida para o mesmo conjunto de dados (porém usando parâmetros empíricos) o qual, foi mostrado por Quina et al. ${ }^{2}$, ser compatível com o processo de transferir um soluto e sua cavidade da fase aquosa até a fase micelar. Para este propósito, é instrutivo comparar a equação (6.2) com a equação (2.7). Comparando as duas equações, podemos esperar que o coeficiente do termo $|q-|$ fosse grande e negativo e que o termo $q_{+}$fosse desprezível. Embora essa expectativa é confirmada para $\mid q$ - $\mid$, o termo $q_{+}$contribui para a equação (6.2), ou seja, é estatisticamente significante. A origem dessa aparente discrepância é solucionada pela análise da correlação do termo $\Sigma \beta_{2}$ da equação (2.7) com o equivalente calculado através das cargas formais, dada pela seguinte relação. 


$$
\begin{gathered}
\Sigma \beta_{2}=0,04+1,22\left|q_{-}\right|-0,62 q_{+} \\
(S D S: n=66: R=0,874 ; s d=0,09 ; F=102,2)
\end{gathered}
$$

Quando a equação (6.3) é levada em consideração, as equações (6.2) e (2.7) levam à mesma conclusão sobre a natureza dos fatores envolvidos na incorporação, pois nas duas equações, (6.2) e (2.7), os termos estatisticamente significativos são $\Sigma \beta_{2}$ e $V_{x} / 100$.

Como era de se esperar, o resultado da análise por regressão linear múltipla, efetuado com a equação (5.25) para o conjunto de dados da Tabela 6.1, mostra novamente que, os coeficientes dos parâmetros teóricos $\varepsilon_{\text {HOMO, }} \varepsilon_{\text {LUMO }},\left\langle\mu>\right.$ e $\pi_{\mathrm{F}}$ da equação (5.25) são essencialmente zero e não são estatisticamente significativos. Este resultado é confirmado através da análise de $s d$ e $F$ parciais, cujos resultados são mostrados na Tabela 6.2.

Tabela 6.2 Resumo da análise estatística (desvio padrão sd e valores de $F$ parcial) para os coeficientes da equação (5.25), mantendo todos os termos, e para a equação final (6.2) onde somente as cargas formais e o termo de cavidade

\begin{tabular}{|c|c|c|c|c|c|c|}
\hline termo & $\begin{array}{l}\text { eq. }(5.25) \\
\text { coeficiente }\end{array}$ & $s d$ & $F$ parcial & $\begin{array}{c}\text { eq. (6.2) } \\
\text { coeficiente }\end{array}$ & $s d$ & $F$ parcial \\
\hline constante & $-1,838$ & 0,746 & & $-0,562$ & 0,088 & \\
\hline$|q|$. & $-2,943$ & 0,424 & 117,681 & $-2,937$ & 0,230 & 117,68 \\
\hline$q_{+}$ & 1,565 & 0,404 & 20,013 & 1,655 & 0,307 & 20 \\
\hline$\pi_{\mathrm{F}}$ & 0,055 & 0,023 & $0,371^{b}$ & & & \\
\hline$\varepsilon_{\text {HOMO }}$ & $-0,067$ & 0,057 & $2,063^{b}$ & & & \\
\hline$\varepsilon_{\text {LUMO }}$ & 0,048 & 0,036 & $3,108^{b}$ & & & \\
\hline$\mu_{\mathrm{CMl}}$ & $-0,003$ & 0,043 & $-0,464^{b}$ & & & \\
\hline $\mathrm{V}_{\mathrm{x}} / 100$ & 2,910 & 0,144 & 594 & 2,998 & 0,09 & 594 \\
\hline
\end{tabular}
são mantidos.

Os parâmetros usados na análise estatística (Tabela 6.1) foram calculados através o hamiltoniano $M N D O-P M 3$. ${ }^{\text {b }}$ Valores estatisticamente nāo significativos.

Note que as duas equações (6.1) e (6.2) são similares, isto é, descrevem o mesmo comportamento, diferindo apenas na magnitude dos coeficientes. A similaridade das equações de regressão (6.1) e (6.2), indica que o tipo de hamiltoniano semi-empírico empregado para calcular os valores dos parâmetros teóricos não é relevante no contexto da metodologia.

A correlação expressa através da equações (6.2), permite estimar os coeficientes de incorporação micelar para uma série de 66 solutos não iônicos com precisão de um fator dois usando parâmetros teóricos como descritores e calculados unicamente a partir da estrutura molecular do soluto.

Em termos matemáticos, os resultados obtidos através de relações lineares teóricas de energia livre de solvatação, são consistentes com os resultados obtidas através de LSERS, usando como descritores, os parâmetros empíricos do soluto tabulados por Abraham.

Na Figura (6.1), mostramos uma comparação entre os valores experimentais de $\left(K_{s}\right)$ e calculados através da equação (6.2). 


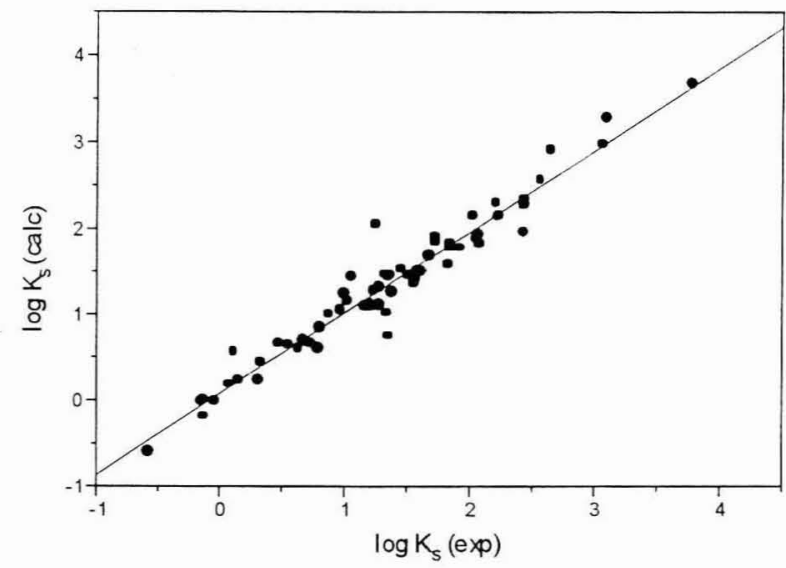

Figura 6.1 Comparação entre os valores experimentais e calculados de $\left(K_{s}\right)$ para a incorporação de (66) solutos não iônicos em micelas aquosas formadas por SDS.

Os resultados mostrados acima, confirmam nossa expectativas quanto ao uso de relações lineares teóricas de energia livre de solvatação para identificar e quantificar os fatores que contribuem para a incorporação de solutos não iônicos em micelas aquosas. A interpretação físico-química das equações de regressão linear teórica será discutida na seção (6.2).

Nosso próximo passo foi aplicar a metodologia para um outro tipo de sistema organizado formado por detergente aniônico. Recentemente, em nosso laboratório Leiva ${ }^{3}$ utilizou métodos fotofísicos, cromatográficos e de solubilização em saturação para determinar valores de $K_{s}$ para a incorporação de solutos não iônicos em agregados de detergente-polímero formado por dodecíl sulfato de sódio $(S D S)$ e poli(etilenoglicol) (PEG) em solução aquosa de $\mathrm{NaCl} 0,1 \mathrm{M}$. Na Tabela 6.3, mostramos os valores de $\log \left(K_{\mathrm{s}}\right)$ determinados por Leiva e os valores dos parâmetros teóricos para cada soluto, usados para descrever a incorporação de 37 solutos não iônicos em micelas aquosas formadas por SDS$P E G$. O resultado da análise por regressão linear múltipla para o conjunto de dados da Tabela 6.3, levando em conta os sete parâmetros teóricos, fornece a seguinte equação de regressão.

$$
\begin{aligned}
\log \left(K_{s}\right)= & -0,27-3,31\left|q_{-}\right|+2,58 q_{+}+0,16 \pi_{\mathrm{F}}+0,22 \varepsilon_{\mathrm{HOMO}}-0,08 \varepsilon_{\mathrm{LUMO}}+ \\
& +0,03<\mu>+2,27\left(\mathrm{~V}_{x} / 100\right)
\end{aligned}
$$

$$
(S D S-P E G: n=37 ; R=0,969 ; s d=0,30 ; F=65)
$$

Analisando a equação de regressão (6.4), observamos que, como no caso das micelas formadas por $S D S$, os coeficientes dos parâmetros $\varepsilon_{\text {HOMO, }} \varepsilon_{\text {LUMO, }}\left\langle\mu\right.$ e $\pi_{\mathrm{F}}$ não são estatisticamente significativos. A equação de regressão final, que melhor descreve o processo de incorporação novamente contém apenas os termos $|q|$, $q_{+}$e $\mathrm{V}_{\mathrm{x}} / 100$. 


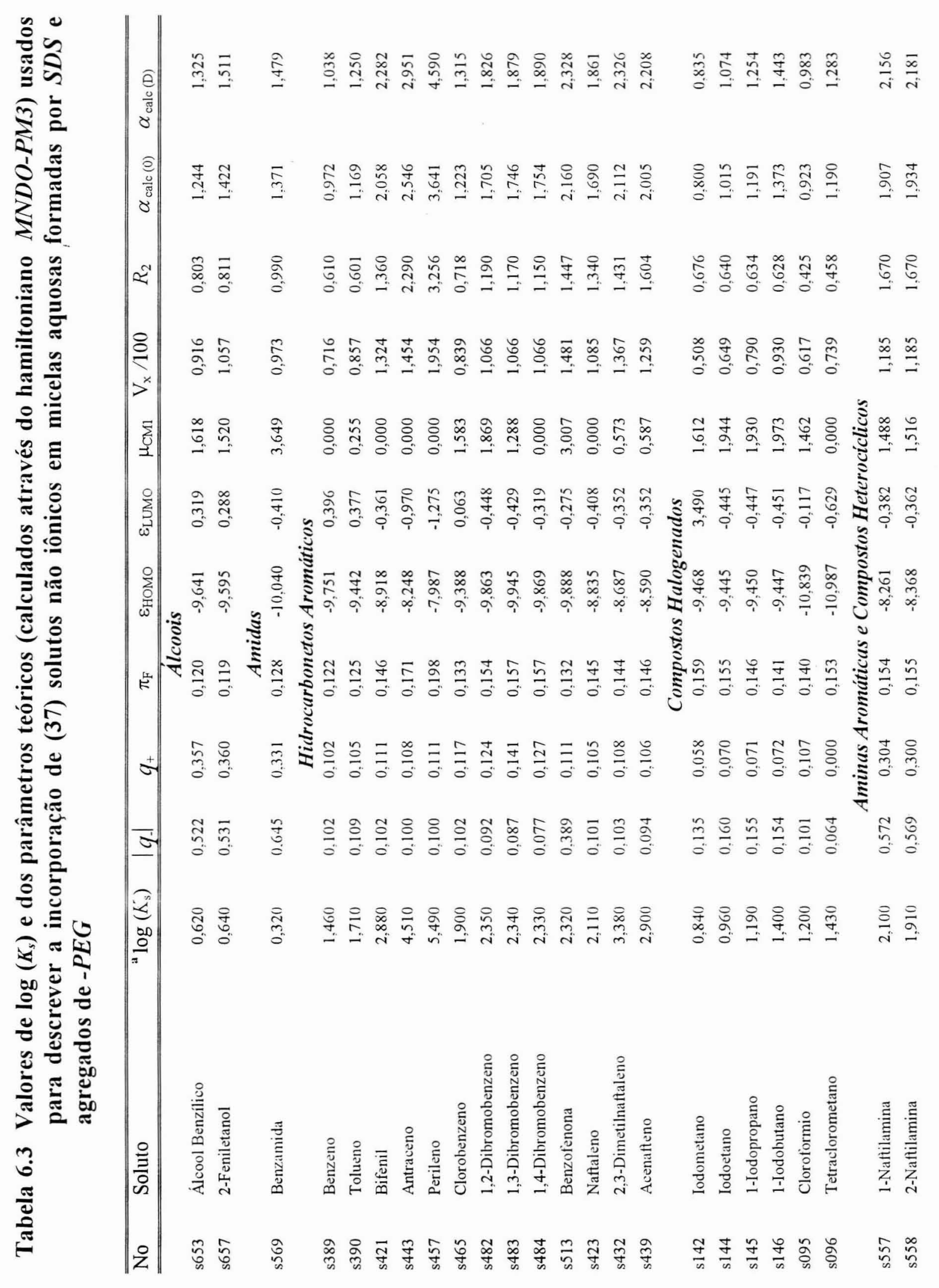




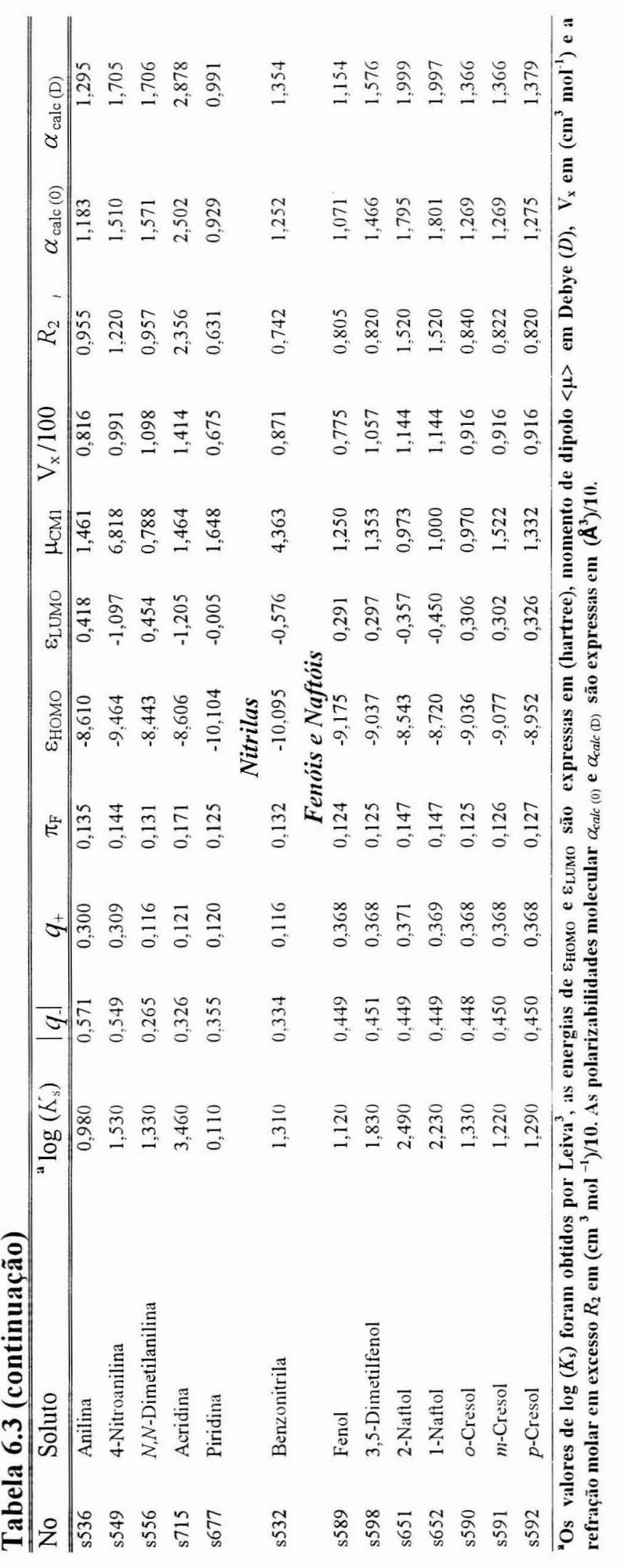




$$
\begin{gathered}
\log \left(K_{s}\right)=-0,81-3.02\left|q_{-}\right|+1,66 q_{+}+3,11\left(\mathrm{~V}_{\mathrm{x}}, 100\right) \\
(S D S-P E G: n=37 ; R=0,938 ; s d=0,37 ; F=81,7)
\end{gathered}
$$

No entanto, comparando a equação (6.5) com as equações (6.1) e (6.2), observa-se que, embora as três equações contenham a mesma informação, a equação (6.5) tem grau de correlação (expresso através valores de $R$ e $F$ ) nitidamente inferior ao das equações (6.1) e (6.2). Porém note que na Tabela 6.3 foram incluídas três novas colunas em comparação com a Tabela 6.1, pois como mencionamos na seção 3.2 a escolha apropriada dos parâmetros a ser usado em um determinado estudo está baseado unicamente na intuição química do pesquisador. Estas colunas mostram os valores tabulado por Abraham para refração molar em excesso $\left(R_{2}\right)$ e a polarizabilidade molecular calculada para os casos de

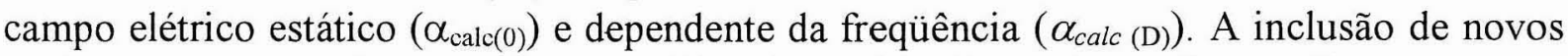
parâmetros no estudo requer uma análise de multicolinearidade. Esta análise mostra a existência ou não de correlação linear simples entre os descritores. Se houver colinearidade significativa entre eles, não haverá uma única solução para a correlação linear múltipla e o modelo perde sua validade. Na Tabela 6.4 , mostramos a matriz de correlação para o conjunto de dados exibidos na Tabela 6.3.

\begin{tabular}{|c|c|c|c|c|c|c|c|c|c|c|}
\hline & $\mid q$. & $q+$ & $\pi_{\mathrm{F}}$ & $\varepsilon_{\text {HOMO }}$ & $\varepsilon_{\text {LUMO }}$ & $\mu_{\mathrm{CMl}}$ & $\mathrm{V}_{\mathrm{x}} / 100$ & $R_{2}$ & $\alpha_{\text {calc (0) }} / 10$ & $\alpha_{\text {calc (D) }} / 10$ \\
\hline$|q|$ & 1,000 & & & & & & & & & \\
\hline$q_{+}$ & 0,858 & 1,000 & & & & & & & & \\
\hline$\pi_{F}$ & $-0,405$ & $-0,411$ & 1,000 & & & & & & & \\
\hline$\varepsilon_{\text {HOMO }}$ & 0,192 & 0,282 & 0,339 & 1,000 & & & & & & \\
\hline$\varepsilon_{\text {LUMO }}$ & 0,042 & 0,077 & -0.362 & $-0,109$ & 1,000 & & & & & \\
\hline$\mu_{\mathrm{CM} 1}$ & 0,487 & 0,230 & $-0,205$ & $-0,275$ & $-0,109$ & 1,000 & & & & \\
\hline $\mathrm{V}_{x} / 100$ & $-0,029$ & 0,005 & 0,537 & 0,600 & $-0,550$ & $-0,188$ & 1,000 & & & \\
\hline$R_{2}$ & $-0,018$ & $-0,003$ & 0,737 & 0,675 & $-0,496$ & $-0,182$ & 0,905 & 1,000 & & \\
\hline$\alpha_{\text {calc (0) }} / 10$ & $-0,127$ & $-0,094$ & 0,700 & 0,591 & $-0,567$ & $-0,226$ & 0.975 & 0.957 & 1,000 & \\
\hline$\alpha_{\text {calc (D) }} 10$ & $-0,125$ & $-0,092$ & 0,719 & 0,596 & $-0,557$ & $-0,226$ & 0,960 & 0,964 & 0,996 & 1,000 \\
\hline
\end{tabular}

Tabela 6.4 Matriz de correlação para os parâmetros teóricos da Tabela 6.3

Analisando a Tabela 6.4 observa-se que há graus de colinearidade significativos entre as variáveis $\mathrm{V}_{\mathrm{x}} / 100$ e $\alpha_{\text {calc (0) }} / 10(0,975), \mathrm{V}_{\mathrm{x}} / 100$ e $\alpha_{\text {calc (D) }} / 10(0,960), R_{2}$ e $\mathrm{V}_{\mathrm{x}} / 100$ $(0,905), R_{2}$ e $\alpha_{\text {calc }(0)} / 10(0,957), R_{2}$ e $\alpha_{\text {calc (D) }} / 10(0,964)$ e $\alpha_{\text {calc (0) }} / 10$ e $\alpha_{\text {calc (D) }} / 10(0,996)$. Como os pares de variáveis correlacionados descrevem o mesmo fato, isto é, tem o mesmo significado, podemos fazer uma mudança de variável e usar $\alpha_{\text {calc (0) }} / 10$ ou $\alpha_{\text {calc (D) }} / 10$ no lugar de $\mathrm{V}_{\mathrm{x}} / 100 \mathrm{e}$, com isso, melhorar o grau de correlação da equação de regressão linear teórica que descreve a incorporação de solutos não iônicos em micelas aquosas formadas por SDS e agregados de PEG. Para esse propósito, mostramos através das equações (6.6) e (6.7), o grau de correlação entre os parâmetros $\mathrm{V}_{\mathrm{x}} / 100$ e $\alpha_{\text {calc (0) }} / 10$ e $\mathrm{V}_{\mathrm{x}} / 100$ e $\alpha_{\text {calc (D) }} / 10$ respectivamente. Sem perda de generalidade podemos fazer a troca de parâmetro, pois o grau de correlação das equações (6.6) e (6.7) descrito por $R$ e $F$ é satisfatório. 


$$
\mathrm{V}_{\mathrm{x}} / 100=0,34+0,39\left(\alpha_{\text {calc (D) }} / 10\right)
$$

$(S D S-P E G: n=37 ; R=0,958 ; s d=0,08 ; F=393,4)$

$$
\begin{gathered}
\mathrm{V}_{\mathrm{x}} / 100=0,23+0,50\left(\alpha_{\mathrm{calc}(0)} / 10\right) \\
(S D S-P E G: n=37 ; R=0,975 ; s d=0,06 ; F=664,9)
\end{gathered}
$$

Quando a mudança de parâmetro é levada em consideração, a equação de regressão linear teórica que melhor correlaciona $\log \left(K_{\varsigma}\right)$ com os parâmetros teóricos, passa a ser descrita através das equações (6.8) e (6.9).

$$
\begin{gathered}
\log \left(K_{s}\right)=-0,05-2,78\left|q_{-}\right|+2,01 q_{+}+1,32\left(\alpha_{\mathrm{calc}(\mathrm{D})} / 10\right. \\
(S D S-P E G: n=37 ; R=0,962 ; s d=0,29 ; F=137,8) \\
\log \left(K_{s}\right)=-0,39-2,78\left|q_{-}\right|+2,03 q_{+}+1,67\left(\alpha_{\text {calc }(0)} / 10\right) \\
(S D S-P E G: n=37 ; R=0,967 ; s d=0,28 ; F=142,7)
\end{gathered}
$$

Comparando as equações (6.8) e (6.9) com a equação (6.5), as equações (6.8) e (6.9) apresentam grau de correlação superior ao da equação (6.5) e grau de correlação compatível ao das equações (6.1) e (6.2) para uma amostragem de 66 solutos. Porém, como a equação (6.9) apresenta grau de correlação superior ao da equação (6.8), consequentemente, esta equação deverá reproduzir os coeficientes de incorporação com maior grau de precisão, pois o valor de $R$ está mais próximo a 1 e o valor de $F$ é maior.

Para que, a nova equação de regressão, usando $\alpha_{\text {calc (0) }} / 10$ como descritor no lugar de $\mathrm{V}_{\mathrm{x}} / 100$ espelhe as propriedades específicas no ambiente de solubilização dos solutos, os valores e os sinais dos coeficientes da equação de regressão linear teórica devem ser compatíveis com os da equação de $L S E R$ para o mesmo conjunto de dados, porém, usando parâmetros determinados experimentalmente. Na Tabela 6.5 mostramos os valores dos parâmetros empíricos do soluto tabulados por $\mathrm{Abraham}^{4}$, para o mesmo conjunto de dados, usados para obter a equação (6.9).

Tabela 6.5 Valores dos parâmetros empíricos do soluto tabulados por Abraham para o conjunto de dados usados para obter a equação de regressão (6.9)

\begin{tabular}{clccccc}
\hline \hline No & Soluto & $\mathrm{R}_{2}$ & $\pi_{2}$ & $\Sigma \beta_{2}$ & $\Sigma \alpha_{2}$ & $V_{\mathrm{X}} / 100$ \\
\hline \hline \multirow{2}{*}{ s653 } & Álcool Benzilico & Álcoois & & & \\
s657 & 2-Feniletanol & 0,803 & 0,870 & 0,560 & 0,330 & 0,916 \\
& & 0,811 & 0,910 & 0,650 & 0,300 & 1,057 \\
s569 & Benzamida & Amidas & & & \\
& & 0,990 & 1,500 & 0,670 & 0,490 & 0,973 \\
s389 & Benzeno & Hidrocarbonetos Aromáticos & & & \\
s390 & Tolueno & 0,610 & 0,520 & 0,140 & 0,000 & 0,716 \\
s421 & Bifenil & 0,601 & 0,520 & 0,140 & 0,000 & 0,857 \\
s443 & Antraceno & 1,360 & 0,990 & 0,220 & 0,000 & 1,324 \\
s457 & Perileno & 2,290 & 1,340 & 0,260 & 0,000 & 1,454 \\
& & 3,256 & 1,760 & 0,400 & 0,000 & 1,954
\end{tabular}


Tabela 6.5 (continuação)

\begin{tabular}{|c|c|c|c|c|c|c|}
\hline No & Soluto & $\mathrm{R}_{2}$ & $\pi_{2}$ & $\Sigma \beta_{2}$ & $\Sigma \alpha_{2}$ & $\mathrm{~V}_{\mathrm{X}} / 100$ \\
\hline s465 & Clorobenzeno & 0,718 & 0,650 & 0,070 & 0,000 & 0,839 \\
\hline s482 & 1,2-Dibromobenzeno & 1,190 & 0,960 & 0,040 & 0,000 & 1,066 \\
\hline s483 & 1,3-Dibromobenzeno & 1,170 & 0,880 & 0,040 & 0,000 & 1,066 \\
\hline s484 & 1,4-Dibromobenzeno & 1,150 & 0,860 & 0,040 & 0,000 & 1,066 \\
\hline s513 & Benzofenona & 1,447 & 1,500 & 0,500 & 0,000 & 1,481 \\
\hline s423 & Naftaleno & 1,340 & 0,920 & 0,200 & 0,000 & 1,085 \\
\hline s432 & 2,3-Dimetilnaftaleno & 1,431 & 0,950 & 0,200 & 0,000 & 1,367 \\
\hline s439 & Acenafteno & 1,604 & 1,050 & 0,200 & 0,000 & 1,259 \\
\hline \multicolumn{7}{|c|}{ Compostos Halogenados } \\
\hline s142 & Iodometano & 0,676 & 0,430 & 0,130 & 0,000 & 0,508 \\
\hline s144 & Iodoetano & 0,640 & 0,400 & 0,150 & 0,000 & 0,649 \\
\hline s145 & 1-Iodopropano & 0,634 & 0,400 & 0,150 & 0,000 & 0,790 \\
\hline s146 & 1-Iodobutano & 0,628 & 0,400 & 0,150 & 0,000 & 0,930 \\
\hline s095 & Cloroformio & 0,425 & 0,490 & 0,020 & 0,150 & 0,617 \\
\hline s096 & Tetraclorometano & 0,458 & 0,380 & 0,000 & 0,000 & 0,739 \\
\hline \multicolumn{7}{|c|}{ Aminas Aromáticas e Compostos Heterocíclicos } \\
\hline s557 & 1-Naftilamina & 1,670 & 1,260 & 0,570 & 0,200 & 1,185 \\
\hline s558 & 2-Naftilamina & 1,670 & 1,280 & 0,550 & 0,220 & 1,185 \\
\hline s536 & Anilina & 0,955 & 0,960 & 0,500 & 0,260 & 0,816 \\
\hline s549 & 4-Nitroanilina & 1,220 & 1,910 & 0,380 & 0,420 & 0,991 \\
\hline s556 & $\mathrm{N}, \mathrm{N}$-Dimetilanilina & 0,957 & 0,840 & 0,420 & 0,000 & 1,098 \\
\hline s715 & Acridina & 2,356 & 1,330 & 0,580 & 0,000 & 1,414 \\
\hline s677 & Piridina & 0,631 & 0,840 & 0,520 & 0,000 & 0,675 \\
\hline \multicolumn{7}{|c|}{ Nitrilas } \\
\hline s532 & Benzonitrila & 0,742 & 1,110 & 0,330 & 0,000 & 0,871 \\
\hline \multicolumn{7}{|c|}{ Fenóis e Naftóis } \\
\hline s589 & Fenol & 0,805 & 0,890 & 0,300 & 0,600 & 0,775 \\
\hline s598 & 3,5-Dimetilfenol & 0,820 & 0,840 & 0,360 & 0,570 & 1,057 \\
\hline s651 & 1-Naftol & 1,520 & 1,050 & 0,370 & 0,610 & 1,144 \\
\hline s652 & 2-Naftol & 1,520 & 1,080 & 0,400 & 0,610 & 1,144 \\
\hline s590 & $o$-Cresol & 0,840 & 0,860 & 0,300 & 0,520 & 0,916 \\
\hline s591 & $m$-Cresol & 0,822 & 0,880 & 0,340 & 0,570 & 0,916 \\
\hline s592 & $p$-Cresol & 0,820 & 0,870 & 0,310 & 0,570 & 0,916 \\
\hline
\end{tabular}

Os valores de $R_{2}, \pi_{2}, \Sigma \beta_{2}, \Sigma \alpha_{2} \mathrm{e} V_{\mathrm{x}} / 100$ foram compilados da referência 4 . As unidades de $R_{2}$ e $V_{\mathrm{x}}$ são expressas em $\left(\mathrm{cm}^{3} \mathrm{~mol}^{-1}\right) / 10$ e $\left(\mathrm{cm}^{3} \mathrm{~mol}^{-1}\right)$ respectivamente. $O$ valor de $\Sigma \beta_{2}$ para Anilina foi substituídos pelo valor alternativo $\Sigma \beta_{2}{ }^{05}$.

O resultado da análise por regressão linear múltipla para o conjunto de dados da Tabela 6.5, fornece a seguinte equação de $L S E R$.

$$
\begin{gathered}
\log \left(K_{s}\right)=-0,34+1,24 R_{2}-0,30 \pi_{2}-2,42 \sum \beta_{2}-0,13 \sum \alpha_{2}+1,75\left(V_{x} / 100\right) \\
(S D S-P E G: n=37 ; R=0,982 ; s d=0,22 ; F=166,6)
\end{gathered}
$$

Agora podemos comparar a equação (6.9) com a equação (6.10). Como no caso das micelas de $S D S$ o termo $\Sigma \beta_{2}$ da equação (6.10) é descrito pelo seu equivalente calculado através das cargas formais dada pela seguinte relação.

$$
\Sigma \beta_{2}=0,07+1,09|q-|-0,40 q_{+}
$$

$(S D S-P E G: n=37 ; R=0,867 ; s d=0,10 ; F=51,6)$ 
Os termos $R_{2}$ e $V_{x} / 100$ da equação (6.10) são descritos pelo equivalente calculado através da polarizabilidade molecular, (incluindo correções atômica) conforme a seguinte relação.

$$
\begin{gathered}
\alpha_{\text {calc }(0)} / 10=-0,08+0,39 R_{2}+1,18\left(V_{x} / 100\right) \\
(S D S-P E G: n=37 ; R=0,991 ; s d=0,08 ; F=912,6)
\end{gathered}
$$

Quando as equações (6.11) e (6.12) são levadas em consideração, as equações (6.9) e (6.10) levam à mesma conclusão sobre a natureza dos fatores envolvidos na incorporação de solutos não iônicos em micelas formadas por $S D S$ e agregados de $P E G$, pois nas duas equações os termos dominantes que contribuem para a incorporação são $R_{2}, \Sigma \beta_{2}$ e $V_{\mathrm{x}} / 100$.

Analisando a equação de regressão final (6.5), observamos que, os coeficientes dos parâmetros teóricos $\varepsilon_{\mathrm{HOMO}}, \varepsilon_{\mathrm{LUMO}}, \varangle>\mathrm{e} \pi_{\mathrm{F}}$ da equação $(5,25)$ não são estatisticamente significativos. Esse fato é novamente confirmado pela análise de $s d$ e $F$ parcial, cujo resultado é mostrado na Tabela 6.6.

Tabela 6.6 Resumo da análise estatística (desvio padrão sd e valores de $F$ parcial) para os coeficientes da equação (6.4), mantendo todos os termos da equação (6.5) onde somente as cargas formais e o termo de cavidade são

\begin{tabular}{|c|c|c|c|c|c|c|c|c|c|}
\hline termo & $\begin{array}{c}\text { eq. (6.4) } \\
\text { coeficiente }\end{array}$ & $s d$ & $F$ parcial & $\begin{array}{c}\text { eq. }(6.5) \\
\text { coeficiente }\end{array}$ & $s d$ & $F$ parcial & $\begin{array}{l}\text { eq. (6.9) } \\
\text { coeficiente }\end{array}$ & $s d$ & $F$ parcial \\
\hline constante & $-0,275$ & 1,460 & & $-0,814$ & 0,26 & & $-0,425$ & 0,17 & \\
\hline$|q|$ & $-3,307$ & 0,631 & 83,6 & $-3,023$ & 0,65 & 42 & $-3,028$ & 0.49 & 48 \\
\hline$q+$ & 2,581 & 0,890 & 9,7 & 1,663 & 1,02 & 9,40 & 2,448 & 0,76 & 86,3 \\
\hline$\pi_{F}$ & 0,160 & 0,004 & $0,014^{b}$ & & & & & & \\
\hline$\varepsilon_{\text {HOMO }}$ & 0,224 & 0,108 & $1,763^{b}$ & & & & & & \\
\hline$\varepsilon_{\text {LUMO }}$ & $-0,081$ & 0,055 & $1,144^{b}$ & & & & & & \\
\hline$\mu_{\mathrm{CMl}}$ & $-0,030$ & 0,052 & $0,498^{b}$ & & & & & & \\
\hline $\mathrm{V}_{\mathrm{x}} / 100$ & 2.270 & 0,291 & 471 & 3,114 & 0.23 & 197 & & & \\
\hline$\alpha_{\text {calc }(0)} / 10$ & & & & & & & 1,708 & 0,09 & 396 \\
\hline
\end{tabular}
mantidos e para equação (6.9) que utiliza o parâmetro $\alpha_{\text {calc (0) }} / 10$.

O fato dos parâmetros teóricos $\varepsilon_{\text {HOMO, }} \varepsilon_{\text {LUMO, }}<\mu>$ e $\pi_{\mathrm{F}}$ não serem estatisticamente significativos, implica que esses parâmetros não são relevantes para reproduzir coeficientes de incorporação $\left(K_{\mathrm{s}}\right)$ de solutos não iônicos em micelas formadas por detergentes aniônicos e consequentemente não devem ser relevantes para descrever processos de incorporação em meio organizado.

Na Figura 6.2, mostramos uma comparação entre os valores experimentais de $\left(K_{s}\right)$ determinados por Leiva e os valores teóricos calculados através da equação (6.9). 


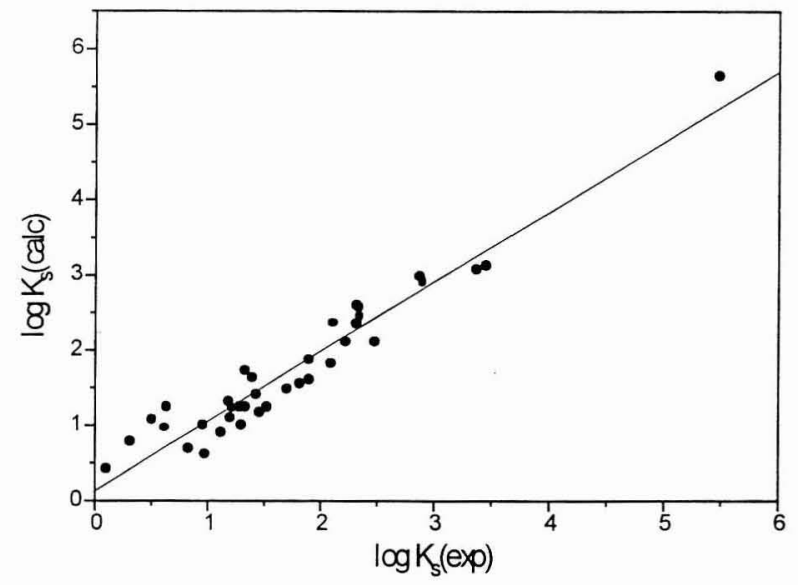

Figura 6.2 Comparação entre os valores experimentais e calculados de $\left(K_{s}\right)$ para a incorporação de (37) solutos não iônicos em micelas aquosas formadas por $S D S$ e agregados de $P E G$.

\subsubsection{Micelas formadas por $D T A B$ e CTAB}

Os resultados apresentados na seção anterior indicam que valores experimentais de $K_{s}$ para incorporação de solutos não iônicos em micelas formadas por detergentes aniônicos podem ser reproduzidos através da técnica de regressão linear múltipla usando parâmetros teóricos como descritores.

Nosso próximo passo foi verificar se a metodologia pode ser aplicada para analisar incorporação de solutos não iônicos em micelas formadas por outros tipos de detergentes. $\mathrm{Na}$ Tabela 6.7, mostramos os valores de $\log \left(K_{s}\right)$ obtidos através de técnicas experimentais e os valores dos parâmetros teóricos usados para descrever a incorporação de 39 solutos não iônicos em micelas aquosas formadas por $D T A B$. O detergente $D T A B$, difere de $S D S$ e $S D S-P E G$ por constituir um sistema catiônico.

O resultado da análise por regressão linear múltipla para o conjunto de dados da Tabela 6.7 mostra que, como no caso dos detergentes aniônicos, os coeficientes dos termos $\varepsilon_{\mathrm{HOMO}}, \varepsilon_{\mathrm{LUMO}},\left\langle\mu e \pi_{\mathrm{F}}\right.$ da equação (5.25) não são estatisticamente significativos e novamente a equação de regressão final contém somente as cargas formais $\left|q_{-}\right|, q_{+}$e o termo de cavidade expresso por $\mathrm{V}_{\mathrm{x}} / 100$. O fato dos parâmetros teóricos $\varepsilon_{\mathrm{HOMO}}, \varepsilon_{\mathrm{LUMO}}, \mu>$ $e \pi_{\mathrm{F}}$ não serem relevantes para descrever incorporação de solutos não iônicos em micelas formadas por detergentes catiônico, indica que esses parâmetros não são relevantes para descrever o processo de incorporação em micelas formadas por detergentes, pois na seção 6.1.1, mostramos que este comportamento também ocorre para a incorporação em micelas formadas por detergentes aniônicos. Porém, ao contrário dos detergentes aniônicos, a equação de regressão final não fornece uma boa correlação, isto é, os valores de $R, s d$ e $F$ não são estatisticamente significativos ao nível de estabelecer uma correlação entre $\log \left(K_{s}\right)$ e os parâmetros teóricos. Este resultado é descrito através da equação (6.13).

$$
\log \left(K_{s}\right)=-0,74-2,83|q-|+2,10 q_{+}+2,91\left(\mathrm{~V}_{x} / 100\right)
$$

$$
(D T A B: n=39 ; R=0,897 ; s d=0,31 ; F=48,1)
$$


Tabela 6.7 Valores de $\log \left(K_{s}\right)$ e dos parâmetros teóricos (calculados através do hamiltoniano $M N D O-P M 3)$ usados para descrever a incorporação de (39) solutos não iônicos em micelas aquosas formadas por DTAB.

\begin{tabular}{|c|c|c|c|c|c|c|c|c|c|}
\hline No & Soluto & ${ }^{\mathrm{a}} \log \left(\mathrm{K}_{\mathrm{s}}\right)$ & $|q|$ & $q+$ & $\pi_{\mathrm{F}}$ & $\varepsilon_{\text {HOMO }}$ & $\varepsilon_{\text {LUMO }}$ & $\mu_{\mathrm{CM} 1}$ & $\mathrm{~V}_{\mathrm{x}} / 100$ \\
\hline \multicolumn{10}{|c|}{ Álcoois } \\
\hline s324 & Metanol & $-0,700$ & 0,528 & 0,355 & 0,085 & $-11,138$ & 3,508 & 1,593 & 0,308 \\
\hline $\mathrm{s} 325$ & Etanol & $-0,260$ & 0,529 & 0,356 & 0,092 & $-11,126$ & 3,358 & 1,607 & 0,449 \\
\hline s326 & 1-Propanol & 0,090 & 0,530 & 0,359 & 0,096 & $-10,883$ & 3,229 & 1,516 & 0,590 \\
\hline s327 & 2-Propanol & 0,130 & 0,530 & 0,359 & 0,096 & $-11,038$ & 3,276 & 1,583 & 0,590 \\
\hline $\mathrm{s} 328$ & 1-Butanol & 0,590 & 0,530 & 0,359 & 0,098 & $-10,887$ & 3,159 & 1,531 & 0,731 \\
\hline s330 & 2-Butanol & 0,380 & 0,534 & 0,363 & 0,098 & $-10,920$ & 3,186 & 1,712 & 0,731 \\
\hline s332 & 1-Pentanol & 0,960 & 0,530 & 0,359 & 0,100 & $-10,889$ & 3,114 & 1,497 & 0,872 \\
\hline s361 & Ciclohexanol & 1,110 & 0,528 & 0,359 & 0,105 & $-10,902$ & 3,154 & 1,564 & 0,904 \\
\hline s347 & 1-heptanol & 1,860 & 0,530 & 0,359 & 0,102 & $-10,891$ & 3,061 & 1,491 & 1,154 \\
\hline s653 & Álcool Benzilico & 1,050 & 0.522 & 0.357 & 0.120 & -9.641 & 0,319 & 1,618 & 0,916 \\
\hline s340 & 1-Hexanol & 1,502 & 0,530 & 0,359 & 0,101 & $-10,890$ & 3,083 & 1,522 & 1,013 \\
\hline s331 & 2-Metil-2-Propanol & 0,190 & 0,525 & 0,359 & 0,098 & $-11,278$ & 3,254 & 1,553 & 0,731 \\
\hline \multicolumn{10}{|c|}{ Aldeídos } \\
\hline $\mathrm{s} 501$ & Benzaldeído & 1,110 & 0,410 & 0,120 & 0,125 & $-10,050$ & $-0,484$ & 2,832 & 0,873 \\
\hline s173 & Butanal & 0,360 & 0,408 & 0,081 & 0,099 & $-10,631$ & 0,832 & 2,765 & 0,688 \\
\hline \multicolumn{10}{|c|}{ Aminas } \\
\hline s272 & Dimetilamina & 0,200 & 0,508 & 0,311 & 0,101 & $-9,219$ & 2,909 & 0,975 & 0,490 \\
\hline s561 & Benzilamina & 0,950 & 0,669 & 0,298 & 0,124 & $-9,455$ & 0,142 & 1,197 & 0,957 \\
\hline s278 & Trietilamina & 1,040 & 0,358 & 0,063 & 0,106 & $-9,012$ & 2,556 & 0,433 & 1,054 \\
\hline \multicolumn{10}{|c|}{ Hidrocarbonetos Aromáticos } \\
\hline s389 & Benzeno & 1,920 & 0,102 & 0,102 & 0,122 & $-9,751$ & 0,396 & 0,000 & 0,716 \\
\hline s465 & Clorobenzeno & 1,810 & 0,102 & 0,117 & 0,133 & $-9,388$ & 0,063 & 1,583 & 0,839 \\
\hline s564 & Nitrobenzeno & 1,400 & 0,109 & 0,134 & 0,124 & $-10,602$ & -1.134 & 5,075 & 0,891 \\
\hline s390 & Tolueno & 1,610 & 0,102 & 0,105 & 0,125 & $-9,442$ & 0,377 & 0,255 & 0,857 \\
\hline \multicolumn{10}{|c|}{ Éster e Éteres } \\
\hline s213 & Acetato de $n$-Propila & 0,700 & 0,467 & 0,071 & 0,101 & $-11,250$ & 1,048 & 2,113 & 0,888 \\
\hline s154 & Éter Dietílico & 0.150 & 0,334 & 0,049 & 0.101 & -10.479 & 2.865 & 1,144 & 0,731 \\
\hline s165 & Tetrahidrofuran & $-0,050$ & 0,341 & 0,057 & 0,101 & $-10,262$ & 3.289 & 1,647 & 0,622 \\
\hline s169 & 1,4-Dioxano & -0.300 & 0,360 & 0,064 & 0,103 & $-10,448$ & 2.840 & 0.000 & 0.681 \\
\hline \multicolumn{10}{|c|}{ Compostos Halogenados } \\
\hline s094 & Diclorometano & 0,710 & 0,364 & 0,076 & 0,124 & $-10,582$ & 0,521 & 1,831 & 0,494 \\
\hline s095 & Cloroformio & 0,920 & 0,348 & 0,107 & 0,140 & $-10,839$ & $-0,117$ & 1,462 & 0,617 \\
\hline s151 & Halotano & 0,980 & 0,137 & 0,138 & 0,119 & $-11,036$ & $-1,420$ & 1,775 & 0,741 \\
\hline s133 & 1-Bromobutano & 1,320 & 0,101 & 0,072 & 0,122 & $-10,925$ & $-0,157$ & 2,025 & 0,847 \\
\hline \multicolumn{10}{|c|}{ Cetonas } \\
\hline s506 & Acetofenona & 1,280 & 0,534 & 0,119 & 0,123 & $-10,008$ & $-0,355$ & 2,989 & 1,014 \\
\hline s182 & Butanona & $-0,050$ & 0,150 & 0,075 & 0,099 & $-10,655$ & 0,807 & 2,877 & 0,688 \\
\hline s181 & Propanona & 0,410 & 0,171 & 0,068 & 0,097 & $-10,773$ & 0,793 & 2,915 & 0,547 \\
\hline s201 & Ciclohexanona & 0,560 & 0,404 & 0,077 & 0,106 & $-10,477$ & 0,853 & 3,046 & 0,861 \\
\hline \multicolumn{10}{|c|}{ Aminas e Nitrilas Aromáticas } \\
\hline s536 & Anilina & 1,110 & 0,407 & 0,300 & 0,135 & $-8,610$ & 0,418 & 1,461 & 0,860 \\
\hline s532 & Benzonitrila & 1,180 & 0,406 & 0,116 & 0,871 & $-10,095$ & 4,363 & $-0,576$ & 0,132 \\
\hline $\mathrm{s} 250$ & 1-Cianopropano & 0,430 & 0,408 & 0,074 & 0,686 & $-11,885$ & 4,024 & 1,407 & 0,103 \\
\hline
\end{tabular}


Tabela 6.7 (continuação)

\begin{tabular}{|c|c|c|c|c|c|c|c|c|c|}
\hline No & Soluto & ${ }^{\mathrm{a}} \log \left(\mathrm{K}_{\mathrm{s}}\right)$ & $q$ & $q+$ & $\pi_{\mathrm{F}}$ & $\varepsilon_{\text {HOMO }}$ & $\varepsilon_{\text {LUMO }}$ & $\mu_{\mathrm{CM} 1}$ & $\mathrm{~V}_{\mathrm{x}} / 100$ \\
\hline \multicolumn{10}{|c|}{ Fenóis e Naftóis } \\
\hline s589 & Fenol & 1,370 & 0,449 & 0,368 & 0,775 & $-9,175$ & 1,250 & 0,291 & 0,124 \\
\hline s645 & 4-Nitrofenol & 1,320 & 0,541 & 0,377 & 0,128 & $-10,169$ & $-1,081$ & 5,236 & 0,949 \\
\hline s652 & 2-Naftol & 2,080 & 0,449 & 0,369 & 0,147 & $-8,720$ & $-0,450$ & 1,000 & 1,144 \\
\hline
\end{tabular}

"Os valores de $\log \left(K_{s}\right)$ foram obtidos a partir da média dos valores experimentais de $\left(K_{\mathrm{s}}\right)$ apresentados na referência ${ }^{2}$, as energi-

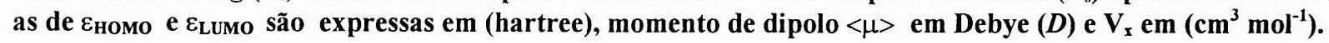

O fato dos valores de $R, s d$ e $F$ para a equação (6.13) não serem estatisticamente significativos ao nível de estabelecer uma correlação entre os parâmetros teóricos e o coeficiente de incorporação $K_{\mathrm{s}}$, levou-nos a não incluir no cálculo da equação de regressão, os solutos cujos resíduos fossem $50 \%$ acima ou a baixo do valor experimental de log $\left(K_{\mathrm{s}}\right)$. Este tipo de procedimento deverá dar-nos subsídios para determinar se a perda da significância estatística, da equação de regressão final, deve-se à maneira pela qual os parâmetros teóricos foram obtidos, ou à escolha do conjunto de parâmetros usados para descrever o processo de incorporação, em micelas formadas por detergentes catiônicos. $\mathrm{O}$ resultado deste procedimento é mostrado na Tabela 6.8 .

Tabela 6.8 DTAB - Resultados da análise por regressão linear múltipla para os coeficientes da equação de regressão final mantendo somente os termos $|q-|, q_{+}$e $V_{\mathrm{x}} / \mathbf{1 0 0}$

\begin{tabular}{|c|c|c|c|c|c|c|c|c|c|c|}
\hline No & Fórmula & ${ }^{d}$ Soluto & $a_{0}$ & $a_{1}$ & $a_{2}$ & $a_{7}$ & $R$ & $n$ & $s d$ & $F$ \\
\hline & & & $-0,74$ & $-2,83$ & $\overline{2,10}$ & 2,91 & 0,897 & 39 & $\overline{0,31}$ & $\overline{48,1}$ \\
\hline s564 & $\mathrm{C}_{6} \mathrm{H}_{5} \mathrm{NO}_{2}$ & Nitrobenzeno & $-0,66$ & $-3,15$ & 2,46 & 2,85 & 0,917 & 38 & 0,28 & 59,9 \\
\hline s169 & $\mathrm{C}_{4} \mathrm{H}_{8} \mathrm{O}_{2}$ & 1,4-Dioxano & $-0,60$ & $-3,05$ & 2,23 & 2,80 & 0,927 & 37 & 0,26 & 65,8 \\
\hline s181 & $\mathrm{C}_{3} \mathrm{H}_{6} \mathrm{O}$ & Propanona & $-0,73$ & $-2,72$ & 2,08 & 2,81 & 0,934 & 36 & 0,24 & 73,5 \\
\hline s389 & $\mathrm{C}_{6} \mathrm{H}_{6}$ & Benzeno & $-0,82$ & $-2,92$ & 2,38 & 2,93 & 0,947 & 35 & 0,22 & 90,9 \\
\hline s536 & $\mathrm{C}_{6} \mathrm{H}_{7} \mathrm{~N}$ & Anilina & $-0,79$ & $-3,04$ & 2,42 & 2,93 & 0,957 & 34 & 0,20 & 108,2 \\
\hline s331 & $\mathrm{C}_{4} \mathrm{H}_{10} \mathrm{O}$ & 2-Metíl-2-Propanol & $-0,78$ & $-3,03$ & 2,51 & 2,91 & 0,963 & 33 & 0,18 & 123,5 \\
\hline s589 & $\mathrm{C}_{6} \mathrm{H}_{6} \mathrm{O}$ & Fenol & $-0,79$ & $-2,95$ & 2,35 & 2,91 & 0,967 & 32 & 0,18 & 134,5 \\
\hline s278 & $\mathrm{C}_{6} \mathrm{H}_{15} \mathrm{~N}$ & Trimetílamina & $-0,84$ & $-2,89$ & 2,20 & 2,98 & 0,971 & 31 & 0,16 & 151 \\
\hline
\end{tabular}

Analisando a Tabela 6.8, nota-se que, para $n=31$, a equação de regressão final passa a ter significância estatística: Logo a perda da significância estatística da equação de regressão final, para $n=39$, é atribuída unicamente a inclusão dos solutos listados na Tabela 6.8. Baseado nesta premissa podemos inicialmente supor que a a natureza da perda de significância estatística deve-se aos valores dos parâmetros teóricos $\left|q_{-}\right|$e $q_{+}$. Estes parâmetros foram obtidos para os solutos listados na Tabela 6.8, pois, quando retiramos estes solutos do cômputo da equação de regressão final, esta passa a ter significância estatística e, quando incluímos, o grau de correlação entre os parâmetros teóricos e o coeficiente de incorporação $K_{s}$, decresce até atingir um nível de insignificância estatística. Na Tabela 6.9 mostramos os valores dos parâmetros teóricos $q_{+}$e $\left|q_{-}\right|$e os valores dos correspondentes parâmetros empíricos $\Sigma \alpha_{2}$ e $\Sigma \beta_{2}$ (tabulados por Abraham) para os solutos listados na Tabela 6.8 . 
Tabela 6.9 Valores dos parâmetros empíricos do soluto $\Sigma \alpha_{2}$ e $\quad \Sigma \beta_{2}$ e dos correspondentes parâmetros teóricos $q_{+}$e $\left|q_{-}\right|$respectivamente.

\begin{tabular}{lllccc|c}
\hline \hline No & Fórmula & Soluto & $\Sigma \alpha_{2}$ & $q+$ & $\Sigma \beta_{2}$ & $q]$ \\
\hline \hline s564 & $\mathrm{C}_{6} \mathrm{H}_{5} \mathrm{NO}_{2}$ & Nitrobenzeno & 0,000 & 0,134 & 0,280 & 0,534 \\
s181 & $\mathrm{C}_{4} \mathrm{H}_{8} \mathrm{O}_{2}$ & Propanona & 0,040 & 0,068 & 0,490 & 0,407 \\
S169 & $\mathrm{C}_{3} \mathrm{H}_{6} \mathrm{O}$ & 1,4-Dioxano & 0,000 & 0,064 & 0,640 & 0,360 \\
s389 & $\mathrm{C}_{6} \mathrm{H}_{6}$ & Benzeno- & 0,000 & 0,102 & 0,140 & 0,102 \\
s536 & $\mathrm{C}_{6} \mathrm{H}_{7} \mathrm{~N}$ & Anilina & 0,260 & 0,300 & 0,500 & 0,571 \\
s331 & $\mathrm{C}_{4} \mathrm{H}_{10} \mathrm{O}$ & 2-Metíl-2-Propanol & 0,310 & 0,359 & 0,600 & 0,525 \\
s589 & $\mathrm{C}_{6} \mathrm{H}_{6} \mathrm{O}$ & Fenol & 0,600 & 0,368 & 0,300 & 0,449 \\
s278 & $\mathrm{C}_{6} \mathrm{H}_{15} \mathrm{~N}$ & Trimetilamina & 0,000 & 0,063 & 0,790 & 0,358 \\
\hline
\end{tabular}

$\mathrm{O}$ valor de $\Sigma \beta_{2}$ para Anilina foi substituídos pelo valor alternativos $\Sigma \beta_{2}{ }^{05}$.

Note que há uma discrepância entre os valores dos parâmetros $\Sigma \alpha_{2}$ e $\Sigma \beta_{2}$ obtidos experimentalmente e os valores calculados para os correspondentes parâmetros teóricos $q_{+}$ e $|q$.$| respectivamente. Essa discrepância confirma nossa suposição de que, a perda de$ significância estatística, deve-se a maneira como os parâmetros teóricos são calculados e não a escolha do conjunto de parâmetros usados para descrever a incorporação. Por outra lado, quando levamos em conta apenas os solutos cujas cargas formais descrevem de forma satisfatória a capacidade doadora e aceptora de pontes de hidrogênio, isto é, para $n=31$, a equação de regressão final passa a ter significância estatística. Este resultado é mostrado através da equação (6.14).

$$
\begin{gathered}
\log \left(K_{s}\right)=-0,84-2,89\left|q_{-}\right|+2,20 q_{+}+2,98\left(\mathrm{~V}_{x} / 100\right) \\
(D T A B: n=31 ; R=0,971 ; s d=0,16 ; F=151)
\end{gathered}
$$

Em analogia ao procedimento adotado para micelas formadas por detergentes aniônicos, podemos comparar a equação (6.14) com a correspondente equação de $L S E R$ para o mesmo conjunto de dados. Na Tabela 6.10, mostramos os valores dos parâmetros empíricos do soluto tabulados por Abraham, para o mesmo conjunto de dados usados para obter a equação (6.14).

Tabela 6.10 Valores dos parâmetros empíricos do soluto tabulados por Abraham para o conjunto de dados usados para obter a equação de regressão

\begin{tabular}{|c|c|c|c|c|c|c|}
\hline No & Soluto & $R_{2}$ & $\pi_{2}$ & $\Sigma \beta_{2}$ & $\Sigma \alpha_{2}$ & $\mathrm{~V}_{\mathrm{X}} / 100$ \\
\hline \multicolumn{7}{|c|}{ Álcoois } \\
\hline s324 & Metanol & 0,278 & 0,440 & 0,470 & 0,430 & 0,308 \\
\hline s325 & Etanol & 0,246 & 0,420 & 0,480 & 0,370 & 0,449 \\
\hline s326 & 1-Propanol & 0,214 & 0,420 & 0,480 & 0,370 & 0,590 \\
\hline s327 & 2-Propanol & 0,212 & 0,360 & 0,560 & 0,330 & 0,590 \\
\hline s328 & 1-Butanol & 0,224 & 0,420 & 0,480 & 0,370 & 0,731 \\
\hline s330 & 2-Butanol & 0,217 & 0,360 & 0,560 & 0,330 & 0,731 \\
\hline s332 & 1-Pentanol & 0,219 & 0,420 & 0,480 & 0,370 & 0,872 \\
\hline$s 361$ & Ciclohexanol & 0,460 & 0,540 & 0,570 & 0,320 & 0,904 \\
\hline s347 & 1-heptanol & 0,211 & 0,420 & 0,480 & 0,370 & 1,154 \\
\hline s653 & Álcool Benzilico & 0,803 & 0,870 & 0,560 & 0,330 & 0,916 \\
\hline
\end{tabular}
(6.14) 
Tabela 6.10 (continuação)

\begin{tabular}{|c|c|c|c|c|c|c|}
\hline No & Soluto & $R_{2}$ & $\pi_{2}$ & $\Sigma \beta_{2}$ & $\Sigma \alpha_{2}$ & $\mathrm{~V}_{\mathrm{X}} / 100$ \\
\hline s340 & 1-Hexanol & $\overline{0,210}$ & 0,420 & 0,480 & 0,370 & 1,013 \\
\hline \multicolumn{7}{|c|}{ Aldeidos } \\
\hline s501 & Benzaldeído & 0,820 & 1,000 & 0,390 & 0,000 & 0,873 \\
\hline s173 & Butanal & 0,187 & 0,650 & 0,450 & 0,000 & 0,688 \\
\hline \multicolumn{7}{|c|}{ Aminas } \\
\hline s272 & Dimetilamina & 0,189 & 0,300 & 0,660 & 0,080 & 0,490 \\
\hline s561 & Benzilamina & 0,829 & 0,880 & 0,720 & 0,100 & 0,957 \\
\hline \multicolumn{7}{|c|}{ Hidrocarbonetos Aromáticos } \\
\hline s465 & Clorobenzeno & 0,718 & 0,650 & 0,070 & 0,000 & 0,839 \\
\hline s390 & Tolueno & 0,601 & 0,520 & 0,140 & 0,000 & 0,857 \\
\hline \multicolumn{7}{|c|}{ Éster e Éteres } \\
\hline s213 & Acetato de $n$-Propila & 0,092 & 0,600 & 0,450 & 0,000 & 0,888 \\
\hline s154 & Éter Dietílico & 0,041 & 0,250 & 0,450 & 0,000 & 0,731 \\
\hline s165 & Tetrahidrofuran & 0,289 & 0,520 & 0,480 & 0,000 & 0,622 \\
\hline \multicolumn{7}{|c|}{ Compostos Halogenados } \\
\hline s094 & Diclorometano & 0,387 & 0,570 & 0,050 & 0,100 & 0,494 \\
\hline s095 & Cloroformio & 0,425 & 0,490 & 0,020 & 0,150 & 0,617 \\
\hline s151 & Halotano & 0,102 & 0,380 & 0,050 & 0,150 & 0,741 \\
\hline s133 & 1-Bromobutano & 0,360 & 0,400 & 0,120 & 0,000 & 0,847 \\
\hline \multicolumn{7}{|c|}{ Cetonas } \\
\hline s506 & Acetofenona & 0,818 & 1,010 & 0,480 & 0,000 & 1,014 \\
\hline s182 & Butanona & 0,166 & 0,700 & 0,510 & 0,000 & 0,688 \\
\hline s201 & Ciclohexanona & 0,403 & 0,860 & 0,560 & 0,000 & 0,861 \\
\hline \multicolumn{7}{|c|}{ Aminas e Nitrilas Aromáticas } \\
\hline s532 & Bezonitrila & 0,742 & 1,110 & 0,330 & 0,000 & 0,871 \\
\hline s250 & 1-Cianopropano & 0,188 & 0,900 & 0,360 & 0,000 & 0,686 \\
\hline \multicolumn{7}{|c|}{ Fenóis e Naftóis } \\
\hline s645 & 4-Nitrofenol & 1,070 & 1,720 & 0,260 & 0,820 & 0,949 \\
\hline s652 & 2-Naftol & 1,520 & 1,080 & 0,400 & 0,610 & 1,144 \\
\hline
\end{tabular}

Os valores de $R_{2}, \pi_{2}, \Sigma \beta_{2}, \Sigma \alpha_{2} \mathrm{e} V_{x} / 100$ foram compilados da referência 4. As unidades de $R_{2}$ e $V_{x}$ são expressas em $\left(\mathrm{cm}^{3} \mathrm{~mol}^{-1}\right) / 10$ e $\left(\mathrm{cm}^{3} \mathrm{~mol}^{-1}\right)$ respectivamente. $O$ valor de $\Sigma \beta_{2}$ para Anilina foi substituídos pelo valor alternativo $\Sigma \beta_{2}{ }^{0}$.

O resultado da análise por regressão linear múltipla para o conjunto de dados da Tabela 6.10 fornece a seguinte equação de $L S E R$.

$$
\begin{gathered}
\log \left(K_{s}\right)=-0,82+0,55 R_{2}-0,49 \pi_{2}-1,57 \sum \beta_{2}+0,14 \sum \alpha_{2}+2,93\left(V_{x} / 100\right) \\
(D T A B: n=31 ; R=0,978 ; s d=0,15 ; F=112,5)
\end{gathered}
$$

Agora podemos comparar a equação (6.14) obtida a partir do conjunto de dados exibidos na Tabela 6.7 porém, excluindo os solutos listados na Tabela 6.9, com a correspondente equação de $L S E R$, obtida a partir do conjunto de dados exibidos da Tabela 6.10. Comparando as duas equações verificamos que os termos dominantes, da equação (6.15), que contribuem para a incorporação são, $\Sigma \beta_{2}$ e $V_{x} / 100$. Como no caso das micelas formadas por detergentes aniônicos, o termo $\Sigma \beta_{2}$ é descrito pelo seu equivalente calculado a partir das cargas formais $|q-|$ e $q_{+}$através da seguinte relação. 


$$
\begin{gathered}
\Sigma \beta_{2}=-0,04+1,35|q-|-0,50 q_{+} \\
(D T A B: n=31: R=0,923 ; s d=0,07 ; F=80,5)
\end{gathered}
$$

Quando as equações (6.14) e (6.16) são levadas em consideração, as equações (6.14) e (6.15) levam a mesma conclusão sobre os fatores envolvidos na incorporação de solutos não iônicos em micelas aquosa formadas por $D T A B$. Na Figura 6.3 mostramos uma comparação entre os valores experimentais de $\left(K_{s}\right)$ e calculados através da equação (6.14).

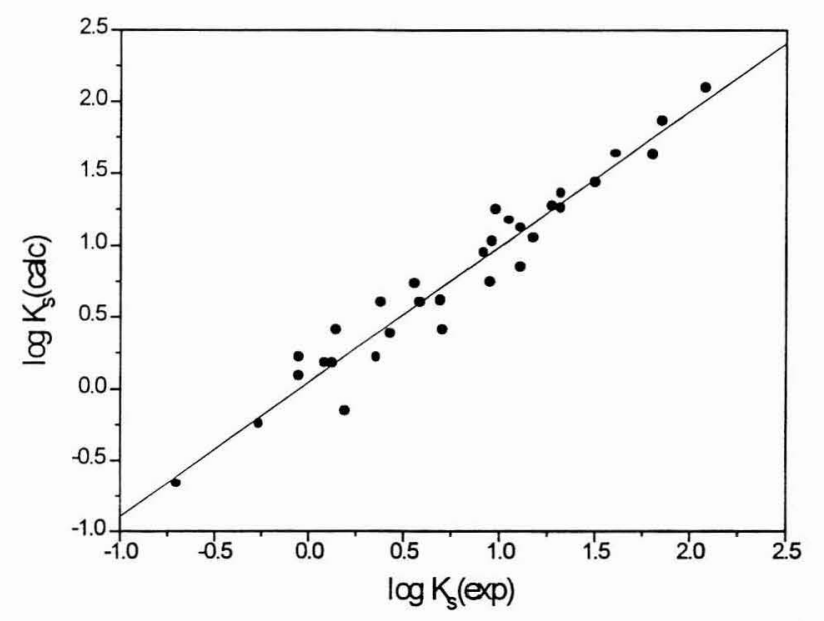

Figura 6.3 Comparação dos valores experimentais e calculados de $\left(K_{s}\right)$ para a incorporação de (31) solutos não iônicos em micelas aquosa formadas por DTAB.

Os resultados apresentado nas Tabelas 6.8 e 6.9, mostram que, para alguns solutos, os valores das cargas formais $\left|q_{-}\right|$e $q_{+}$, calculadas através de métodos semi-empíricos usando a definição de análise populacional de Mulliken, não descrevem de forma satisfatória o caráter doador e aceptor por pontes de hidrogênio. Este resultado mostra particularidades da aplicação da metodologia em estudos de incorporação de solutos não iônicos em micelas formadas por detergentes catiônicos. Nosso próximo passo foi verificar se o comportamento apresentado pelas cargas formais $\left|q_{-}\right|$e $q_{+}$, também ocorre quando o sistema em estudo é composto por micelas formadas por outros detergentes catiônicos, como por exemplo $C T A B$. Na Tabela 6.11, mostramos os valores experimentais de $\log \left(K_{s}\right)$ e os valores dos parâmetros teóricos usados para descrever a incorporação de 50 solutos não iônicos em micelas aquosas formadas por $C T A B$.

O resultado da análise regressão linear múltipla para o conjunto de dados da Tabela 6.11 mostra que, como no caso das micelas formadas por detergentes aniônicos e DTAB, os coeficientes dos parâmetros $\varepsilon_{\mathrm{HOMO}}, \varepsilon_{\mathrm{LUMO}},\left\langle\mu \rho \pi_{\mathrm{F}}\right.$ da equação (5.25) não são estatisticamente significativos e a equação de regressão final contém somente os termos $|q-|$ e $q_{+}$e $V_{\mathrm{x}} / 100$.

$$
\begin{gathered}
\log \left(K_{s}\right)=-0,52-3,02|q-|+0,68 q_{+}+3,44\left(\mathrm{~V}_{x} / 100\right) \\
(C T A B: n=50 ; R=0,960 ; s d=0,37 ; F=181,7)
\end{gathered}
$$


Tabela 6.11 Valores de $\log \left(K_{s}\right)$ e dos parâmetros teóricos (calculados através do hamiltoniano $M N D O-P M 3)$ usados para descrever a incorporação de (50) solutos não iônicos em micelas aquosas formadas por CTAB.

\begin{tabular}{|c|c|c|c|c|c|c|c|c|c|}
\hline No & Soluto & ${ }^{\mathrm{a}} \log \left(K_{\mathrm{s}}\right)$ & $q-1$ & $q_{+}$ & $\pi_{\mathrm{F}}$ & $\varepsilon_{\text {HOMO }}$ & $\varepsilon_{\text {LIMMO }}$ & $\mu_{\mathrm{CMI}}$ & $\mathrm{V}_{\mathrm{X}} / 100$ \\
\hline \multicolumn{10}{|c|}{ Álcoois } \\
\hline s326 & 1-Propanol & $-0,301$ & 0,530 & 0,359 & 0,096 & $-10,883$ & 3,229 & 1,516 & 0,590 \\
\hline s327 & 2-Propanol & $-0,398$ & 0,530 & 0,359 & 0,096 & $-11,038$ & 3,276 & 1,583 & 0,590 \\
\hline $\mathrm{s} 328$ & 1-Butanol & 0,462 & 0,530 & 0,359 & 0,098 & $-10,887$ & 3,159 & 1,531 & 0,731 \\
\hline s330 & 2-Butanol & 0,146 & 0,534 & 0,363 & 0,098 & $-10,920$ & 3,186 & 1,712 & 0,731 \\
\hline $\mathrm{s} 331^{-}$ & 2-Metil 2-Propanol & 0,000 & 0,525 & 0,359 & 0,098 & $-11,278$ & 3,254 & 1,553 & 0,731 \\
\hline s340 & 1-Hexanol & 1,415 & 0,530 & 0,359 & 0,101 & $-10,890$ & 3,083 & 1,522 & 1,013 \\
\hline $\mathrm{s} 361$ & Ciclohexanol & 0,792 & 0,528 & 0,359 & 0,105 & $-10,902$ & 3,154 & 1,564 & 0,904 \\
\hline $\mathrm{s} 653$ & Álcool Benzílico & 1,146 & 0,522 & 0,357 & 0,120 & $-9,641$ & 0,319 & 1,618 & 0,916 \\
\hline \multicolumn{10}{|c|}{ Aldeídos e Cetonas } \\
\hline $\mathrm{s} 501$ & Benzaldeído & 1,301 & 0,410 & 0,120 & 0,125 & $-10,050$ & $-0,484$ & 2,832 & 0,873 \\
\hline $\mathrm{s} 512$ & Etil Feníl Cetona & 1,690 & 0,404 & 0,118 & 0,122 & $-9,999$ & $-0,318$ & 2,890 & 1,155 \\
\hline s506 & Acetofenona & 1,342 & 0,408 & 0,119 & 0,123 & $-10,008$ & $-0,355$ & 2,989 & 1,014 \\
\hline \multicolumn{10}{|c|}{ Hidrocarbonetos Alifáticos } \\
\hline s015 & Metano & 0,279 & 0,000 & 0,000 & 0,084 & $-13,642$ & 4,245 & 0,000 & 0,250 \\
\hline s016 & Etano & 0,939 & 0,000 & 0,000 & 0,094 & $-11,976$ & 3,892 & 0,000 & 0,390 \\
\hline s017 & Propano & 1,518 & 0,000 & 0,000 & 0,097 & $-11,506$ & 3,715 & 0,000 & 0,531 \\
\hline s049 & Ciclohexano & 2,698 & 0,000 & 0,000 & 0,107 & $-11,290$ & 3,476 & 0,000 & 0,845 \\
\hline \multicolumn{10}{|c|}{ Amidas } \\
\hline s569 & Benzamida & 1,041 & 0,645 & 0,331 & 0,128 & $-10,040$ & $-0,410$ & 3,649 & 0,973 \\
\hline \multicolumn{10}{|c|}{ Hidrocarbonetos Aromáticos e Derivados } \\
\hline s389 & Benzeno & 1,602 & 0,102 & 0,102 & 0,122 & $-9,751$ & 0,396 & 0,000 & 0,716 \\
\hline s390 & Tolueno & 2,049 & 0,109 & 0,105 & 0,125 & $-9,442$ & 0,377 & 0,255 & 0,857 \\
\hline s391 & Etilbenzeno & 2,576 & 0,115 & 0,112 & 0,122 & $-9,444$ & 0,388 & 0,300 & 0,998 \\
\hline$s 421$ & Bifenil & 3,176 & 0,102 & 0,111 & 0,146 & $-8,918$ & $-0,361$ & 0,000 & 1,324 \\
\hline s443 & Antraceno & 4,633 & 0,100 & 0,108 & 0,171 & $-8,248$ & $-0,970$ & 0,000 & 1,454 \\
\hline s465 & Clorobenzeno & 2,113 & 0,102 & 0,117 & 0,133 & $-9,388$ & 0,063 & 1,583 & 0,839 \\
\hline$s 481$ & Bromobenzeno & 2,297 & 0,097 & 0,123 & 0,141 & $-9,806$ & $-0,051$ & 1,423 & 0,891 \\
\hline$s 423$ & Naftaleno & 3,176 & 0,101 & 0,105 & 0,145 & $-8,835$ & $-0,408$ & 0,000 & 1,085 \\
\hline $\mathrm{s} 453$ & Pireno & 5,223 & 0,101 & 0,106 & 0,183 & $-7,585$ & $-0,684$ & 0,000 & 1,585 \\
\hline s457 & Perileno & 5,653 & 0,100 & 0,111 & 0,198 & $-7,987$ & $-1,275$ & 0,000 & 1,954 \\
\hline s564 & Nitrobenzeno & 1,591 & 0,534 & 0,134 & 0,124 & $-10,602$ & $-1,134$ & 5,075 & 0,891 \\
\hline \multicolumn{10}{|c|}{ Aminas e Nitrilas Aromáticas } \\
\hline s536 & Anilina & 1,342 & 0,571 & 0,300 & 0,135 & $-8,610$ & 0,418 & 1,461 & 0,816 \\
\hline s539 & $p$-Toluidina & 1,623 & 0,570 & 0,299 & 0,136 & $-8,361$ & 0,458 & 1,304 & 0,957 \\
\hline $\mathrm{s} 532$ & Benzonitrila & 1,322 & 0,334 & 0,116 & 0,132 & $-10,095$ & $-0,576$ & 4,363 & 0,871 \\
\hline s543 & 4-Cloroanilina & 2,114 & 0,566 & 0,301 & 0.148 & -8.584 & 0,103 & 2,848 & 0,939 \\
\hline \multicolumn{10}{|c|}{ Ácidos Carboxilicos } \\
\hline s580 & Ácido Benzóico & 2,146 & 0,519 & 0,397 & 0,122 & $-10,133$ & $-0,533$ & 2,386 & 0,932 \\
\hline s583 & 4-Metil Ácido Benzóico & 2,505 & 0,520 & 0,396 & 0,125 & $-9,865$ & $-0,522$ & 2,724 & 1,073 \\
\hline s584 & 4-Etil Ácido Benzóico & 3,041 & 0,520 & 0,396 & 0,124 & $-9,860$ & $-0,509$ & 2,825 & 1,214 \\
\hline \multicolumn{10}{|c|}{ Ésteres $e$ Éteres } \\
\hline s514 & Benzoato de Metila & 1,716 & 0,463 & 0,119 & 0,121 & $-10,075$ & $-0,353$ & 2,285 & 1,073 \\
\hline $\mathrm{s} 515$ & Benzoato de Etíla & 2,301 & 0,468 & 0,119 & 0,121 & $-10,058$ & $-0,326$ & 2,283 & 1,214 \\
\hline $\mathrm{s} 552$ & 4-Aminobenzoato de Metíla & 2,397 & 0,562 & 0,304 & 0,134 & $-8,900$ & $-0,297$ & 3,161 & 1,313 \\
\hline s490 & Anisole & 1,663 & 0,287 & 0,120 & 0,126 & $-9,140$ & 0,338 & 1,080 & 0,916 \\
\hline
\end{tabular}


Tabela 6.11 (continuação)

\begin{tabular}{|c|c|c|c|c|c|c|c|c|c|}
\hline No & Soluto & ${ }^{\mathrm{a}} \log \left(K_{\mathrm{s}}\right)$ & $q-1$ & $q_{+}$ & $\pi_{\mathrm{F}}$ & $\varepsilon_{\text {HOMO }}$ & $\varepsilon_{\text {LUMO }}$ & $\mu_{\mathrm{CM} 1}$ & $\mathrm{~V}_{\mathrm{X}} / 100$ \\
\hline \multicolumn{10}{|c|}{ Gases } \\
\hline s010 & Oxigênio & $-0,143$ & 0,000 & 0,000 & 0,053 & $-10,733$ & $-0,982$ & 0,000 & 0,183 \\
\hline s008 & Argônio & $-0,060$ & 0,000 & 0,000 & & & & & 0,190 \\
\hline \multicolumn{10}{|c|}{ Compostos Halogenados } \\
\hline s146 & 1-Iodobutano & 2,653 & 0,154 & 0,072 & 0,141 & $-9,447$ & $-0,451$ & 1,973 & 0,930 \\
\hline s094 & Diclorometano & 0,763 & 0,137 & 0,076 & 0,124 & $-10,582$ & 0,521 & 1,831 & 0,494 \\
\hline s095 & Cloroformio & 1,415 & 0,101 & 0,107 & 0,140 & $-10,839$ & $-0,117$ & 1,462 & 0,617 \\
\hline s090 & Tetrafluorometano & 0,519 & 0,136 & 0,000 & 0,047 & $-16,789$ & 0,506 & 0,000 & 0,320 \\
\hline s096. & Tetraclorometano & 2,000 & 0,064 & 0,000 & 0,153 & $-10,987$ & $-0,629$ & 0,000 & 0,739 \\
\hline \multicolumn{10}{|c|}{ Fenóis e Naftóis } \\
\hline s589 & Fenol & 1,845 & 0,449 & 0,368 & 0,124 & $-9,175$ & 0,291 & 1,250 & 0,775 \\
\hline s592 & $p$-Cresol & 2,230 & 0,450 & 0,368 & 0,127 & $-8,952$ & 0,326 & 1,332 & 0,916 \\
\hline$s 601$ & 4-Etíl Fenol & 2,591 & 0,450 & 0,368 & 0,125 & $-8,953$ & 0,337 & 1,411 & 1,057 \\
\hline s609 & 4-Propíl Fenol & 2,991 & 0,449 & 0,368 & 0,123 & $-8,958$ & 0,331 & 1,420 & 1,198 \\
\hline$s 615$ & 4-Butíl Fenol & 3,114 & 0,449 & 0,368 & 0,123 & $-8,989$ & 0,356 & 1,353 & 1,339 \\
\hline
\end{tabular}

${ }^{a}$ Os valores de $\log \left(K_{\mathrm{s}}\right)$ foram obtidos através da média dos valores apresentados nas referência ${ }^{2,6}$, as energias de $\varepsilon_{H O M O}$ e $\varepsilon_{L U M O}$ são expressas em (hartree), momento de dipolo $\mu_{C M 1}$ em Debye (D) e $V_{\mathrm{x}} \mathrm{em}\left(\mathrm{cm}^{3} \mathrm{~mol}^{-1}\right)$.

Novamente, os valores de $R$, sd e $F$ indicam um grau apenas aceitável de correlação entre os parâmetros teóricos log $\left(K_{s}\right)$. Porém, exatamente como no caso das micelas formadas por $D T A B$, quando retiramos do cômputo da equação de regressão final os solutos cujos resíduos são $50 \%$ acima ou a baixo do valor experimental de $\log \left(K_{\mathrm{s}}\right)$, a significância estatística da correlação é resgatada. O resultado deste procedimento é mostrado na Tabela 6.12 .

Tabela 6.12 CTAB - Resultados da análise por regressão linear múltipla para os coeficientes da equação final mantendo somente as cargas formais $\left|q_{-}\right|$e $q_{+}$e o termo de cavidade $V_{x} / 100$.

\begin{tabular}{lllcccccccc}
\hline \hline No & Fórmula & Soluto & $\mathrm{a}_{0}$ & $\mathrm{a}_{1}$ & $\mathrm{a}_{2}$ & $\mathrm{a}_{7}$ & $R$ & $n$ & $s d$ & $F$ \\
\hline \hline & & $-0,52$ & $-3,02$ & 0,68 & 3,44 & 0,960 & 50 & 0,37 & 181 \\
$\mathrm{~s} 589$ & $\mathrm{C}_{6} \mathrm{H}_{6} \mathrm{O}$ & Fenol & $-0,54$ & $-3,04$ & 0,57 & 3,48 & 0,964 & 49 & 0,36 & 199,8 \\
$\mathrm{~s} 580$ & $\mathrm{C}_{7} \mathrm{H}_{6} \mathrm{O}_{2}$ & Ácido Benzóico & $-0,55$ & -3.10 & 0,47 & 3,49 & 0,968 & 48 & 0,34 & 223 \\
$\mathrm{~s} 592$ & $\mathrm{C}_{7} \mathrm{H}_{8} \mathrm{O}$ & $p$-Cresol & $-0,55$ & $-3,12$ & 0,38 & 3,51 & 0,972 & 47 & 0,32 & 251,3 \\
$\mathrm{~s} 583$ & $\mathrm{C}_{8} \mathrm{H}_{8} \mathrm{O}_{2}$ & 4-Metíl Ácido Benzóico & $-0,54$ & $-3,18$ & 0,29 & 3,51 & 0,976 & 46 & 0,30 & 284,7 \\
$\mathrm{~s} 536$ & $\mathrm{C}_{6} \mathrm{H}_{7} \mathrm{~N}$ & Anilina & $-0,54$ & $-3,29$ & 0,28 & 3,54 & 0,979 & 45 & 0,28 & 329,5 \\
$\mathrm{~s} 564$ & $\mathrm{C}_{6} \mathrm{H}_{5} \mathrm{NO}_{2}$ & Nitrobenzeno & $-0,54$ & $-3,45$ & 0,39 & 3,53 & 0,983 & 44 & 0,26 & 387,3 \\
$\mathrm{~s} 539$ & $\mathrm{C}_{7} \mathrm{H}_{9} \mathrm{~N}$ & p-Toluídina & $-0,53$ & $-3,56$ & 0,40 & 3,54 & 0,986 & 43 & 0,24 & 448,8 \\
$\mathrm{~s} 584$ & $\mathrm{C}_{9} \mathrm{H}_{10} \mathrm{O}_{2}$ & 4-Etíl Ácido Benzóico & $-0,50$ & $-3,62$ & 0,43 & 3,50 & 0,988 & 42 & 0,22 & 524,6 \\
$\mathrm{~s} 361$ & $\mathrm{C}_{6} \mathrm{H}_{12}$ & Ciclohexanol & $-0,52$ & $-3,65$ & 0,43 & 3,55 & 0,989 & 41 & 0,21 & 571,1 \\
$\mathrm{~s} 017$ & $\mathrm{C}_{3} \mathrm{H}_{8}$ & Propano & $-0,454$ & $-3,68$ & 0,34 & 3,58 & 0,991 & 40 & 0,20 & 647,2 \\
\hline
\end{tabular}

$\overline{\log \left(K_{s}\right)=a_{11}+a_{1}|q-|+a_{2} q_{+}+a_{3} \pi_{F}+a_{4} \varepsilon_{H O M O}+a_{5} \varepsilon_{L U M O}+a_{6}<\mu>+a_{7}(V x / 100) . ~}{ }^{d}$ Solutos não inclusos na equação de regressão final.

Este resultado mostra novamente que, para alguns solutos, os parâmetros $\left|q_{-}\right|$e $q_{+}$, calculadas via método semi-empírico, não podem ser associados a capacidade doadora e aceptora de pontes de hidrogênio, quando o sistema em estudo é composto por micelas formadas por detergentes catiônicos pois o valor calculado para as cargas formais $\left|q_{-}\right|$e $q_{+}$ não reflete este caráter de forma adequada. Quando levamos em conta apenas os solutos 
cujas cargas formais $|q-|$ e $q$ - descrevem de forma satisfatória a capacidade doadora e aceptora de pontes de hidrogênio, isto é, para $n=40$, a equação de regressão final passa a ter significância estatística sendo descrita pela equação (6.18).

$$
\begin{gathered}
\log \left(K_{s}\right)=-0,54-3,58|q-|+0,34 q_{+}+3,58\left(\mathrm{~V}_{x} / 100\right) \\
(C T A B: n=40 ; R=0,991 ; s d=0,20 ; F=647,2)
\end{gathered}
$$

Em analogia ao procedimento adotado nas seções anteriores, devemos comparar a equação (6.18) com a correspondente equação de $L S E R$ para o mesmo conjunto de dados. Na tabela 6.13, mostramos os valores dos parâmetros empíricos do soluto tabulados por Abraham para o conjunto de dados usados para obter a equação (6.18).

Tabela 6.13 Valores dos parâmetros empíricos do soluto tabulados por Abraham para

\begin{tabular}{|c|c|c|c|c|c|c|}
\hline No & Soluto & $R_{2}$ & $\pi_{2}$ & $\Sigma \beta_{2}$ & $\sum \alpha_{2}$ & $\mathrm{~V}_{\mathrm{X}} / 100$ \\
\hline \multicolumn{7}{|c|}{ Alcoois } \\
\hline s326 & 1-Propanol & 0,370 & 0,480 & 0.214 & 0,420 & 0,590 \\
\hline$\$ 327$ & 2-Propanol & 0,330 & 0,560 & 0,212 & 0,360 & 0,590 \\
\hline s328 & 1-Butanol & 0,370 & 0,480 & 0,224 & 0,420 & 0,731 \\
\hline$\$ 330$ & 2-Butanol & 0,330 & 0,560 & 0,217 & 0,360 & 0,731 \\
\hline s331 & 2-Metil 2-Propanol & 0,310 & 0,600 & 0,180 & 0,300 & 0,731 \\
\hline$s 340$ & 1-Hexanol & 0,370 & 0,480 & 0,210 & 0.420 & 1,013 \\
\hline$\$ 361$ & Ciclohexanol & 0.320 & 0.570 & 0.460 & 0.540 & 0.904 \\
\hline s653 & Álcool Benzilico & 0,330 & 0,560 & 0,803 & 0.870 & 0,916 \\
\hline \multicolumn{7}{|c|}{ Aldeidos e Cetonas } \\
\hline s501 & Benzaldeido & 0,000 & 0,390 & 0.820 & 1,000 & 0,873 \\
\hline s506 & Acetofenona & 0,000 & 0,480 & 0,818 & 1,010 & 1.014 \\
\hline \multicolumn{7}{|c|}{ Hidrocarbonetos Alifáticos } \\
\hline s015 & Metano & 0,000 & 0.000 & 0.000 & 0.000 & 0.250 \\
\hline$\leqslant 016$ & Etano & 0.000 & 0.000 & 0.000 & 0.000 & 0.390 \\
\hline s017 & Propano & 0,000 & 0,000 & 0,000 & 0,000 & 0.531 \\
\hline s(0)49 & Ciclohexano & 0.000 & 0.000 & 0.305 & 0.100 & 0.845 \\
\hline s569 & Benzamida & $\begin{array}{c}\text { Amid } \\
0,490\end{array}$ & 0,670 & 0,990 & 1.500 & 0,973 \\
\hline \multicolumn{7}{|c|}{ Hidrocarbonetos Aromáticos e Derivados } \\
\hline s389 & Benzeno & 0.000 & 0.140 & 0,610 & 0.520 & 0,716 \\
\hline s 390 & Tolueno & 0,000 & 0,140 & 0,601 & 0.520 & 0,857 \\
\hline s391 & Etilbenzeno & 0.000 & 0.150 & 0.613 & 0.510 & 0.998 \\
\hline s421 & Bifenil & 0,000 & 0,220 & 1,360 & 0,990 & 1,324 \\
\hline s443 & Antraceno & 0,000 & 0,260 & 2,290 & 1,340 & 1,454 \\
\hline s465 & Clorobenzeno & 0,000 & 0,070 & 0.718 & 0,650 & 0,839 \\
\hline$\$ 481$ & Bromobenzeno & 0.000 & 0.090 & 0.882 & 0.730 & 0.891 \\
\hline$s 423$ & Naftaleno & 0,000 & 0,200 & 1,340 & 0,920 & 1,085 \\
\hline $\mathrm{s} 453$ & Pireno & 0,000 & 0,290 & 2,808 & 1,710 & 1,585 \\
\hline$\$ 457$ & Perileno & 0,000 & 0,400 & 3,256 & 1,760 & 1.954 \\
\hline \multicolumn{7}{|c|}{ Aminas e Nitilas Aromáticas } \\
\hline s532 & Benzonitrila & 0,000 & 0,330 & 0,742 & 1,110 & 0,871 \\
\hline
\end{tabular}
o conjunto de dados usados para obter a equação de regressão (6.18) 
Tabela 6.13 (continuação)

\begin{tabular}{|c|c|c|c|c|c|c|}
\hline No & Soluto & $R_{2}$ & $\pi_{2}$ & $\sum \beta_{2}$ & $\sum \alpha_{2}$ & $\mathrm{~V}_{\mathrm{X}} / 100$ \\
\hline s543 & 4-Cloroanilina & 0,300 & 0.350 & 1.060 & 1.130 & 0,939 \\
\hline \multicolumn{7}{|c|}{ Ésteres e Eteres } \\
\hline s514 & Benzoato de Metíla & 0.000 & 0.460 & 0.733 & 0,850 & 1,073 \\
\hline 8515 & Benzoato de Etíla & 0.000 & 0.460 & 0.689 & 0.850 & 1.214 \\
\hline$\$ 552$ & 4-Aminobenzoato de Metila & 0,320 & 0.640 & 1.040 & 1.520 & 1.313 \\
\hline$s 490$ & Anisole & 0,000 & 0.290 & 0.708 & 0.750 & 0.916 \\
\hline \multicolumn{7}{|c|}{ Gases } \\
\hline$\$ 010$ & Oxigênio & 0,000 & 0,000 & 0.000 & 0.000 & 0.183 \\
\hline s009- & Argônio & 0,000 & 0.000 & 0.000 & 0.000 & 0.190 \\
\hline \multicolumn{7}{|c|}{ Compostos Halogenados } \\
\hline$\$ 146$ & 1-Iodobutano & 0,000 & 0,150 & 0,628 & 0.400 & 0,930 \\
\hline s094 & Diclorometano & 0,100 & 0,050 & 0.387 & 0.570 & 0.494 \\
\hline s095 & Cloroformio & 0,150 & 0,020 & 0,425 & 0.490 & 0,617 \\
\hline s090 & Tetrafluorometano & 0,000 & 0,000 & $-0,280$ & -0.200 & 0,320 \\
\hline$\$(096$ & Tetraclorometano & 0.000 & 0.000 & 0.458 & 0.380 & 0.739 \\
\hline \multicolumn{7}{|c|}{ Fenóis e Naftóis } \\
\hline$s 601$ & 4-Etíl Fenol & 0,550 & 0,360 & 0.800 & 0.900 & 1,057 \\
\hline$\$ 609$ & 4-Propil Fenol & 0.550 & 0.370 & 0.793 & 0.880 & 1.198 \\
\hline s616 & 4-Butil Fenol & 0,796 & 0,890 & 0,380 & 0.550 & 1,339 \\
\hline
\end{tabular}

Os valores de $R_{2}, \pi_{2}, \Sigma \beta_{2}, \sum \alpha_{2} \mathrm{e} V_{\mathrm{x}} / 100$ foram compilados da referência 4 . As unidades de $R_{2}$ e $V_{\mathrm{x}}$ sĩo expressas em $\left(\mathrm{cm}^{3} \mathrm{~mol}^{\mathrm{I}}\right) / 10$ e $\left(\mathrm{cm}^{3} \mathrm{~mol}^{-1}\right)$ respectivamente. $O$ valor de $\Sigma \beta_{2}$ para Anilina foi substituídos peio valor alternativo $\Sigma \beta_{2}{ }^{0}$.

O resultado da análise por regressão linear múltipla para o conjunto de dados da Tabela 6.13, fornece a seguinte equação de $L S E R$.

$$
\begin{gathered}
\log \left(K_{s}\right)=-0,82+0,36 R_{2}-0,02 \pi_{2}-3,69 \sum \beta_{2}+0,78 \sum \alpha_{2}+3,70\left(V_{x} / 100\right) \\
(C T A B: n=40 ; R=0,992 ; s d=0,18 ; F=462,6)
\end{gathered}
$$

Comparando a equação (6.18) com a equação (6.19), verifica-se que, os termos dominantes que contribuem para a incorporação são, $\Sigma \beta_{2}$ e $V_{x} / 100$. Como no caso das micelas formadas por detergentes aniônicos e por $D T A B$, o termo $\Sigma \beta_{2}$ é descrito pelo seu equivalente calculado a partir das cargas formais através da seguinte relação.

$$
\begin{gathered}
\Sigma \beta_{2}=-0,03+0,87\left|q_{-}\right|+0,20 q_{+} \\
(C T A B: n=40: R=0,933 ; s d=0,08 ; F=124,6)
\end{gathered}
$$

Na Figura 6.4, mostramos uma comparação entre os valores experimentais de $\left(K_{\mathrm{s}}\right)$ e os valores teóricos calculados a partir da equação (6.18) 


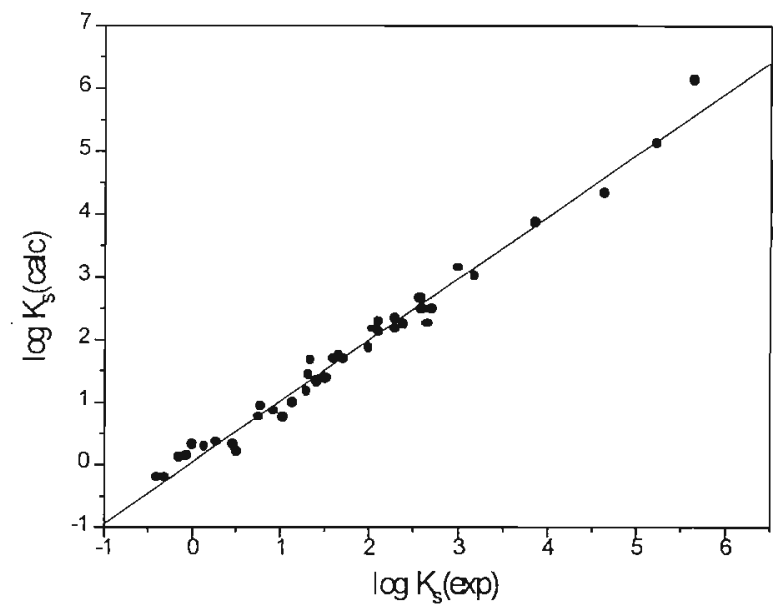

Figura 6.4 Comparação dos valores experimentais e calculados de $\left(K_{s}\right)$ para a incorporação de (40) solutos não iônicos em micelas aquosas formadas por $C T A B$.

Os resultados apresentados nesta seção revela uma particularidade quanto ao uso da metodologia, relacionada com os valores calculados para os parâmetros teóricos $\left|q_{-}\right|$e $q_{+}$. Exatamente como no caso das micelas formadas por $D T A B$, quando não incluímos no cômputo da equação de regressão final, os solutos cujas cargas formais não descrevem de forma satisfatória o caráter doador e aceptor por pontes de hidrogênio, as equações de regressão linear teórica levam a mesma conclusão sobre a natureza dos fatores envolvidos no processo de incorporação.

Como o principal objetivo deste estudo é investigar a potencialidade de uso do enfoque de Famini, para prever eficácias de incorporação, nosso próximo passo foi investigar a aplicação da metodologia para a incorporação de solutos não iônicos em micelas formadas por detergente não iônicos.

\subsubsection{Micelas formadas por Brij-35}

$\mathrm{Na}$ Tabela 6.14, mostramos os valores experimentais de $\log \left(K_{\mathrm{s}}\right)$ e os valores dos parâmetros teóricos usados para descrever a incorporação de 27 solutos não iônicos em micelas aquosas formadas pelo detergentes não iônico Brij-35.

Tabela 6.14 Valores experimentais de $\log \left(K_{s}\right)$ e dos parâmetros teóricos usados para descrever a incorporação de (27) solutos não iônicos em micelas aquosas formadas por Brij-35.

\begin{tabular}{|c|c|c|c|c|c|c|c|}
\hline No & Soluto & ${ }^{2} \log \left(K_{\mathrm{s}}\right)$ & $q-\mid$ & $q_{+}$ & $\mathrm{V}_{\mathrm{x}} / 100$ & $R_{2}$ & $\alpha_{\text {calc }(0)}$ \\
\hline \multicolumn{8}{|c|}{ Alcoois, Aldeidos e Amidas } \\
\hline s653 & Álcool Benzílico & 1,217 & 0,522 & 0,357 & 0,916 & 0,803 & 1,244 \\
\hline s501 & Benzaldeído & 1.255 & 0,410 & 0,120 & 0,873 & 0,820 & 1,210 \\
\hline s569 & Benzamida & 1,513 & 0,645 & 0,331 & 0,973 & 0,990 & 1,371 \\
\hline \multicolumn{8}{|c|}{ Hidrocarbonetos Alifáticos } \\
\hline s015 & Metano & 0,373 & 0,000 & 0,000 & 0,250 & 0,000 & 0,230 \\
\hline
\end{tabular}


Tabela 6.14 (continuação)

\begin{tabular}{|c|c|c|c|c|c|c|c|}
\hline No & Soluto & ${ }^{2} \log \left(K_{\mathrm{s}}\right)$ & $q-\mid$ & $q_{+}$ & $\mathrm{V}_{\mathrm{x}} / 100$ & $R_{2}$ & $\alpha_{\text {calc }(0)}$ \\
\hline s017 & Propano & $1 . \overline{550}$ & 0,000 & 0,000 & 0.531 & $\overline{0,000}$ & 0,587 \\
\hline s016 & Etano & 1,000 & 0,000 & 0,000 & 0,390 & 0,000 & 0,412 \\
\hline \multicolumn{8}{|c|}{ Hidrocarbonetos Aromáticos e Derivados } \\
\hline s389 & Benzeno & 1.576 & 0,102 & 0,102 & 0,716 & 0,610 & 0,972 \\
\hline s390 & Tolueno & 1,984 & 0,109 & 0,105 & 0,857 & 0,601 & 1,169 \\
\hline s391 & Etílbenzeno & 2,464 & 0,115 & 0,112 & 0,998 & 0,613 & 1,349 \\
\hline s481 & Bromobenzeno & 2.547 & 0,097 & 0,123 & 0,891 & 0,882 & 1,352 \\
\hline$s 421$ & Bifenil & 3,650 & 0,102 & 0,111 & 1,324 & 1,360 & 2,058 \\
\hline 5443 & Antraceno & 4.600 & 0,100 & 0,108 & 1,454 & 2,290 & 2,546 \\
\hline s564 & Nitrobenzeno & 1,744 & 0,534 & 0,134 & 0,891 & 0,871 & 1,243 \\
\hline s465 & Clorobenzeno & 2.456 & 0,102 & 0,117 & 0,839 & 0,718 & 1,223 \\
\hline s395 & $n$-Propíl Benzeno & 2,970 & 0,112 & 0,112 & 1,139 & 0,604 & 1,533 \\
\hline \multicolumn{8}{|c|}{ Ácidos Carboxilicos, Éteres e Cetonas } \\
\hline s303 & Ácido Butanóico & 0,663 & 0,519 & 0,394 & 0,747 & 0,210 & 0,849 \\
\hline s304 & Ácido Pentanóico & 1.230 & 0,516 & 0,395 & 0.888 & 0,205 & 1,029 \\
\hline s305 & Ácido 3-Metíl Butanóico & 1,089 & 0,520 & 0,394 & 0,888 & 0,178 & 1,020 \\
\hline s306 & Ácido Hexanóico & 1,699 & 0,519 & 0,394 & 1,028 & 0,174 & 1,203 \\
\hline s506 & Acetofenona & 1.415 & 0.408 & 0,119 & 1.014 & 0,818 & 1,384 \\
\hline s514 & Metílbenzoato & 1,799 & 0,463 & 0,119 & 1,073 & 0,733 & 1,478 \\
\hline s490 & Éter Metíl Feníl & 1,756 & 0,287 & 0,120 & 0,916 & 0,708 & 1,274 \\
\hline \multicolumn{8}{|c|}{ Gases e Compostos Halogenados } \\
\hline s010 & Oxigênio & 0,086 & 0,000 & 0,000 & 0,183 & 0,000 & 0,117 \\
\hline s009 & Argônio & 0,000 & & & & & \\
\hline s096 & Tetraclorometano & 1,960 & 0,064 & 0,000 & 0,739 & 0,458 & 1,190 \\
\hline \multicolumn{8}{|c|}{ Nitrilas e Fenóis } \\
\hline$s 532$ & Benzonitrila & 1,290 & 0,334 & 0,116 & $0,87 \mathrm{I}$ & 0,742 & 1,252 \\
\hline s589 & Fenol & 2,318 & 0,449 & 0,368 & 0,775 & 0,805 & 1,071 \\
\hline
\end{tabular}

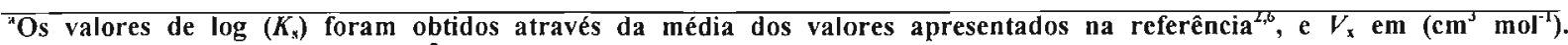
A polarizabilidade $\alpha_{\text {calc(i) }} / 10$ em $\left(\AA^{3}\right)$ e $R_{2} \mathrm{em}\left(\mathrm{cm}^{3} \mathrm{~mol}^{-1}\right) / 10$.

O resultado da análise por regressão linear múltipla, para o conjunto de dados da Tabela 6.14 mostra que, como no caso dos detergentes aniônicos e catiônicos, os coeficientes dos parâmetros $\varepsilon_{\mathrm{HOMO}}, \varepsilon_{\mathrm{LUMO}}, \mu>e \pi_{\mathrm{F}}$ não são estatisticamente significativos e, a equação final contém somente as cargas formais e o termo de cavidade.

$$
\begin{gathered}
\log \left(K_{s}\right)=-0,40-2,96|q-|+1,11 q_{+}+3,28\left(\mathrm{~V}_{x} / 100\right) \\
(\text { Brij-35: } n=27 ; R=0,943 ; s d=0,35 ; F=61,6)
\end{gathered}
$$

Novamente como no caso das micelas formadas por detergentes cationicos, os valores de $R, s d$ e $F$ para a equação (6.21) indicam um grau apenas razoável de correlação entre log $\left(K_{\mathrm{s}}\right)$ e os parâmetros teóricos usados para descrever a incorporação. Note que na Tabela 6.14 mostramos os valores dos parâmetros teóricos $|q-|, q_{+}, V_{x} / 100, R_{2}$ e $\alpha_{\text {calc (0) }} / 10$ por que, exatamente como no caso das micelas formadas por $S D S-P E G$, devemos trocar o parâmetro $V_{x} / 100$ por $\alpha_{\text {calc (0) }} / 10$, quando o termo que descreve a refração molar em excesso tem contribuição significativa na equação de $L S E R$, pois o parâmetro teórico equivalente que descreve esta contribuição é o termo de polarizabilidade molecular $\alpha_{\text {calc (0) }}$ 110. Quando a troca de parâmetro é levada em consideração, a equação de regressão final 
que descreve a incorporação de 27 solutos não iônicos em micelas formadas por detergentes não iônicos passa a ser descrito pela seguinte equação.

$$
\begin{gathered}
\log \left(K_{s}\right)=0,05-2,69\left|q_{-}\right|+1,92 q_{+}+1,82\left(\alpha_{\text {calc }(0)} / 10\right) \\
(B r i j-35: n=27 ; R=0,960 ; s d=0,29 ; F=91,1)
\end{gathered}
$$

Porém, ao contrário das equações que descrevem a incorporação de solutos não iônicos em micelas aquosas formadas por detergentes aniônicos, os valores de $R, s d$ e $F$, indicam um grau apenas aceitável de correlação entre $\log \left(K_{\mathrm{s}}\right)$ e os parâmetros teóricos. Porém, como no caso das micelas aquosas formadas por detergentes cationicos, quando retiramos da equação de regressão final os solutos cujos resíduos são $50 \%$ acima ou abaixo do valor experimental de $\log \left(K_{s}\right)$, a significância estatística da equação de regressão final é resgatada. O resultado deste procedimento é mostrado na Tabela 6.15.

Tabela 6.15 Brij-35 - Resultados da análise por regressão linear múltipla para os coeficientes da equação de regressão final mantendo somente as cargas formais e o termo de polarizabilidade molecular.

\begin{tabular}{lllcccccccc}
\hline \hline No & Fórmula & d Soluto & $\mathrm{a}_{0}$ & $\mathrm{a}_{1}$ & $\mathrm{a}_{2}$ & $\mathrm{a}_{7}$ & $R$ & $n$ & $s d$ & $F$ \\
\hline \hline & & & 0,04 & $-2,69$ & 1,92 & 1,83 & 0,960 & 27 & 0.29 & 91.1 \\
$s 589$ & $\mathrm{C}_{6} \mathrm{H}_{6} \mathrm{O}$ & Fenol & 0.03 & -2.50 & 1,25 & 1.86 & 0.975 & 26 & 0,23 & 144,5 \\
$s 564$ & $\mathrm{C}_{6} \mathrm{H}_{5} \mathrm{NO}_{2}$ & Nitrobenzeno & 0.03 & $-3,12$ & 2,11 & 1,86 & 0,984 & 25 & 0,19 & 219,7 \\
$s 017$ & $\mathrm{C}_{3} \mathrm{H}_{8}$ & Propano & $-0,04$ & $-3,07$ & 2,17 & 1,89 & 0,988 & 24 & 0,17 & 284,4 \\
$s 465$ & $\mathrm{C}_{6} \mathrm{H}_{5} \mathrm{Cl}$ & Clorobenzeno & $-0,10$ & $-3,03$ & 2,21 & 1,92 & 0.989 & 23 & 0,16 & 308.5 \\
$s 016$ & $\mathrm{C}_{2} \mathrm{H}_{6}$ & Etano & $-0,12$ & -2.93 & 2,11 & 1,91 & 0,991 & 22 & 0,15 & 337,8 \\
\hline
\end{tabular}

$\log \left(K_{s}\right)=a_{0}+a_{1}\left|q_{-}\right|+a_{2} q_{+}+a_{3} \pi_{F}+a_{4} \varepsilon_{H O M o}+a_{s} \varepsilon_{L U M O}+a_{6}<\mu>+a_{7}\left(\alpha_{c a l c(0)} / 10\right) .{ }^{d}$ Solutos nāo inclusos na equação de regressão tinal.

O resultado apresentado na Tabela 6.15 revela novamente que as cargas formais $|q-| \mathrm{e}$ $q_{+}$para alguns solutos não refletem de forma satisfátoria a capacidade doadora e aceptora de pontes de hidrogênio. Quando levamos em conta apenas os solutos cuja carga formal descrevem de forma satisfatória a capacidade doadora e aceptora de pontes de hidrogênio, isto é, para $n=22$, a equação de regressão final passa a ter significância estatística sendo descrita pela equação (6.23).

$$
\log \left(K_{s}\right)=-0,12-2,93\left|q_{-}\right|+2,11 q_{+}+1,91\left(\alpha_{\text {calc }(0)} / 10\right)
$$

$$
(\text { Brij-35B: } n=22 ; R=0,991 ; s d=0,15 ; F=337,8)
$$

Em analogia ao procedimento adotado para micelas formadas por detergentes aniônicos e catiônicos, podemos comparar a equação (6.23) com a correspondente equação de LSER para o mesmo conjunto de dados. Na Tabela 6.16, mostramos os valores dos 
parâmetros empíricos do soluto tabulados por Abraham, para o mesmo conjunto de dados usados para obter a equação (6.23).

Tabela 6.16 Valores dos parâmetros empíricos do soluto tabulados por Abraham para o conjunto de dados usados para obter a equação de regressão (6.23)

\begin{tabular}{|c|c|c|c|c|c|c|}
\hline No & Soluto & $R_{2}$ & $\pi_{2}$ & $\Sigma \beta_{2}$ & $\sum \alpha_{2}$ & $\mathrm{~V}_{\mathrm{X}} / 100$ \\
\hline \multicolumn{7}{|c|}{ Álcoois, Aldeídos e Amidas } \\
\hline 5653 & Alcool Benzílico & 0.803 & 0.870 & 0.330 & 0.560 & 0,916 \\
\hline$\$ 501$ & Benzaldeido & 0,820 & 1,000 & 0,000 & 0.390 & 0,873 \\
\hline$s 569$ & Benzamida & 0.990 & 1.500 & 0.490 & 0.670 & 0.973 \\
\hline \multicolumn{7}{|c|}{ Hidrocarboneto Alifático } \\
\hline s015 & Metano & 0,000 & 0,000 & 0,000 & 0.000 & 0,250 \\
\hline \multicolumn{7}{|c|}{ Hidrocarbonetos Aromáticos e Derivados } \\
\hline s389 & Benzeno & 0,610 & 0,520 & 0,000 & 0,140 & 0,716 \\
\hline s390 & Tolueno & 0,601 & 0,520 & 0,000 & 0,140 & 0,857 \\
\hline$\$ 391$ & litillbenzeno & 0.613 & 0.510 & 0.000 & 0.150 & 0.998 \\
\hline s395 & n-Propil Benzeno & 0,604 & 0,500 & 0,000 & 0,150 & 1,139 \\
\hline$s 481$ & Bromobenzeno & 0,882 & 0,730 & 0,000 & 0,090 & 0,891 \\
\hline$s 506$ & Acetofenona & 0,818 & 1,010 & 0,000 & 0,480 & 1,014 \\
\hline $\mathrm{s} 514$ & Metílbenzoato & 0,733 & 0,850 & 0,000 & 0.460 & 1,073 \\
\hline$s 421$ & Bifenil & 1,360 & 0,990 & 0,000 & 0,220 & 1,324 \\
\hline$s 443$ & Antraceno & 2,290 & 1,340 & 0,000 & 0,260 & 1,454 \\
\hline \multicolumn{7}{|c|}{ Acidos Carboxilicos, Eteres e Cetonas } \\
\hline s303 & Ácido Butanóico & 0,210 & 0,620 & 0,600 & 0,450 & 0.747 \\
\hline s304 & Acido Pentanóico & 0,205 & 0,600 & 0.600 & 0,450 & 0,888 \\
\hline$\$ 305$ & Acido 3-Metil Butanóico & 0,178 & 0,570 & 0.600 & 0.490 & 0,888 \\
\hline s306 & Ácido Hexanóico & 0,174 & 0,600 & 0,600 & 0,450 & 1,028 \\
\hline$s 490$ & Éter Metíl Feníl & 0,708 & 0,750 & 0,000 & 0.290 & 0,916 \\
\hline \multicolumn{7}{|c|}{ Gases e Compostos Halogendos } \\
\hline 5008 & Argoninio & 0.000 & 0.000 & 0.000 & 0.000 & 0.190 \\
\hline 5010 & Oxigènio & 0,000 & 0,000 & 0.000 & 0.000 & 0,183 \\
\hline :(096 & Tetraclorometano & 0.458 & 0.380 & 0.000 & 0.000 & 0.739 \\
\hline \multicolumn{7}{|c|}{ Nitrila } \\
\hline$\$ 532$ & Benzonitrila & 0,742 & 1,110 & 0,000 & 0,330 & 0,871 \\
\hline
\end{tabular}

Os valores de $R_{2}, \pi_{2}, \Sigma \beta_{2}, \Sigma \alpha_{2} \mathrm{e} V_{\mathrm{x}}^{\prime} / 100$ foram compilados da referência 4 . As unidades de $R_{2}$ e $V_{x}^{\prime}$ sĩo expressas em (cm $\left.{ }^{3} \mathrm{~mol}^{-1}\right) / 10$ e $\left(\mathrm{cm}^{3} \mathrm{~mol}^{-1}\right)$ respectivamente. $O$ valor de $\Sigma \beta_{2}$ para Anilina foi substituidos pelo valor alternativo $\Sigma \beta_{2}{ }^{0}$.

O resultado da análise por regressão linear múltipla para o conjunto de dados da Tabela 6.16, fornece a seguinte equação de LSER.

$$
\begin{gathered}
\log \left(K_{s}\right)=-0,44+0,84 R_{2}-0,21 \pi_{2}-3,14 \sum \beta_{2}+0,80 \sum \alpha_{2}+2,93\left(V_{x} / 100\right) \\
(B r i j-35: n=22 ; R=0,995 ; s d=0,12 ; F=316,9)
\end{gathered}
$$


Agora podemos comparar a equação (6.23) com a correspondente equação de (LSER) para o mesmo conjunto de dados, comparando as equações $(6.23)$ e $(6,24)$, os termos dominantes que contribuem para a incorporação são $R_{2}, \Sigma \beta_{2}$ e $V_{x} / 100$. Como no caso das micelas aniônicas e cationicas o termo $\Sigma \beta_{2}$ é descrito pelo seu equivalente calculado através da carga formal através da seguinte relação.

$$
\begin{gathered}
\sum \beta_{2}=0,04+0,95\left|q_{-}\right|-0,07 q_{+} \\
(B r i j-35: n=22: R=0,965 ; s d=0,05 ; F=128,1)
\end{gathered}
$$

e como no caso das micelas formadas por SDS-PEG, os termos $R_{2}$ e $V_{\mathrm{x}} / 100$ da equação (6.24) são descritos pelo equivalente calculado através da polarizabilidade molecular conforme a seguinte relação;

$$
\begin{gathered}
\alpha_{\text {calc }(0)} / 10=-0,08+0,39 R_{2}+1,19\left(V_{x} 100\right) \\
(n=22: R=0,996 ; s d=0,06 ; F=792,8)
\end{gathered}
$$

Quando as equações (6.25) e (6.26) são levadas em consideração, as equações (6.23) e (6.24) levam à mesma conclusão sobre os fatores envolvidos na incorporação de solutos não iônicos em micelas aquosa formadas por Brij-35. Na Figura 6.5 mostramos uma comparação entre os valores experimentais de $\log (K \mathrm{~s})$ e os valores teóricos calculados a partir da equação (6.23).

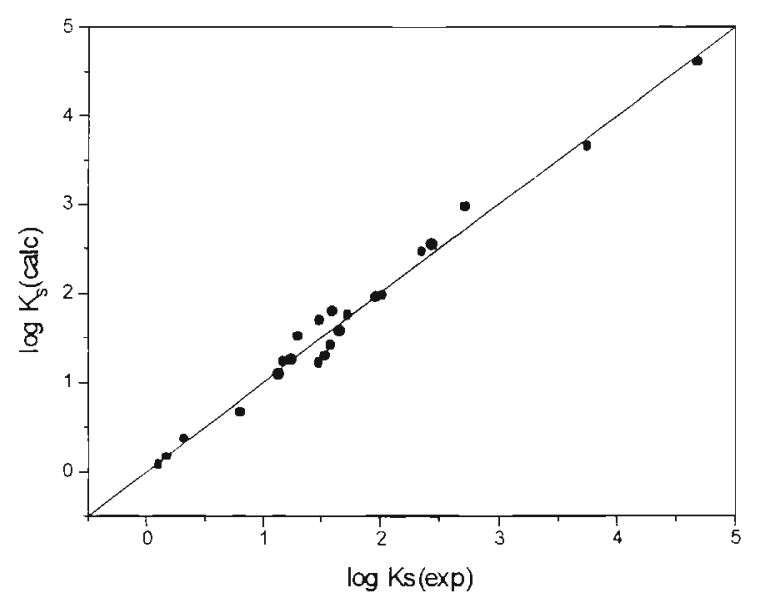

Figura 6.5 Comparação dos valores experimentais e calculados de $\left(K_{s}\right)$ para a incorporação de (22) solutos não iônicos em micelas formadas por Brịj-35.

Em resumo, os resultados obtidos neste estudo confirma nossa expectativa de usar relações lineares teóricas de energia livre de solvatação para elucidar e quantificar os fatores envolvidos no processo de incorporação de solutos não iônicos em micelas aquosas formadas por detergentes aniônicos, catiônicos e não iônicos. As particularidades apresentadas no caso da incorporação em micelas formadas por detergentes catiônicos e não iônicos, será discutido na próxima seção 6.2 e na seção seguinte discutiremos o significado físico-químico das equações de regressão linear teórica. 


\subsection{Limitações da Metodologia}

Os resultados mostrados nas seções 6.1 .2 e 6.1.3 indicam claramente que, quando incluímos no cômputo das equações de regressão final os solutos listados nas Tabelas 6.8, 6.12 e 6.15, o grau de correlação entre os parâmetros teóricos e o coeficiente de incorporação $K_{\mathrm{s}}$, decresce até atingir um nível de insignificância estatística. A natureza desse comportamento foi inicialmente associada ao fato de que os parâmetros teóricos $|q-|$ e $q_{+}$calculados a partir de hamiltonianos semi-empíricos e usando a definição de análise populacional de Mulliken são incapazes de refletir de forma satisfatória a capacidade doadora e aceptora de pontes de hidrogênio, o que se torna importante em sistemas compostos de micelas aquosas formadas por detergentes catiônicos e não iônicos. Do ponto de vista do formalismo matemático, este comportamento está intimamente ligado ao fato dos métodos semi-empíricos empregarem funções de base mínima no cômputo da análise populacional de Mulliken, e por isso não conseguem descrever de forma satisfatória uma distribuição de carga que possa ser associada as capacidades doadora e aceptora de pontes de hidrogênio.

No intuito de remover esta fragilidade da metodologia, uma alternativa seria manter o enfoque de Famini, porém fazer uso de cargas formais proveniente de método ab-initio devido a flexibilidade oferecida na escolha do conjunto de funções de base, assim como pelo rigor e a precisão de cálculo oferecido por este método. Quanto mais completo for o conjunto de funções de base melhor será descrito a distribuição e a transferência de carga envolvida na formação da molécula. Desta forma esperamos, melhorar a performance da metodologia. Neste sentido, calculamos e analisamos os valores das cargas formais obtidas através de método ab-initio usando funções de base 3-21G para o conjunto fechado de 693 solutos não iônicos distribuídos em vários grupos funcionais (veja a Tabela 5.4). Porém, as cargas formais obtidas a partir da definição de análise populacional de Mulliken, e calculada através de método ab-initio, apresentam o mesmo comportamento das cargas formais calculadas usando hamiltonianos semi-empírico, diferindo apenas na magnitude, ou seja, ambas as maneiras de calcular cargas formais são incapazes de revelar sutilezas na distribuição da carga de forma que possamos associar a magnitude das cargas formais $|q-| \mathrm{e}$ $q_{+}$com a magnitude dos parâmetros empíricos $\Sigma \alpha_{2}$ e $\Sigma \beta_{2}$, respectivamente. Na Tabela 6.13 mostramos, para alguns casos, o comportamento das cargas formais calculadas através de métodos semi-empírico e ab-initio.

Tabela 6.17 Valores dos parâmetros empíricos de Abraham e dos correspondentes parâmetros teóricos usados para descrever a capacidade doadora e aceptora de pontes de hidrogênio.

\begin{tabular}{llcccc|c|c|c}
\hline \hline No & Soluto & ${ }^{c} \sum \alpha_{2}$ & $q_{+}$ & ${ }^{c} q_{+}$ & ${ }^{c} \sum \beta_{2}$ & $\left|q_{-}\right|$ & $q_{-} \mid$ \\
\hline & & 0,430 & Alcoois & & & & \\
s324 & Metanol & 0,355 & 0,375 & 0,470 & 0,528 & 0,684 \\
s325 & Etanol & 0,370 & 0,356 & 0,375 & 0,480 & 0,529 & 0,681 \\
s326 & 1-Propanol & 0,370 & 0,359 & 0,374 & 0,480 & 0,530 & 0,685 \\
s327 & 2-Propanol & 0,330 & 0,359 & 0,373 & 0,560 & 0,530 & 0,679 \\
s328 & 1-Butanol & 0,370 & 0,359 & 0,373 & 0,480 & 0,530 & 0,685 \\
s330 & 2-Butanol & 0,330 & 0,363 & 0,370 & 0,560 & 0,534 & 0,672 \\
s332 & 1-Pentanol & 0,370 & 0,359 & 0,374 & 0,480 & 0,530 & 0,686 \\
s333 & 2-Pentanol & 0,330 & 0,359 & 0,380 & 0,560 & 0,530 & 0,686
\end{tabular}


Tabela 6.17 (continuação)

\begin{tabular}{|c|c|c|c|c|c|c|c|}
\hline No & Soluto & ${ }^{c} \sum \alpha_{2}$ & $q_{+}$ & ${ }^{\mathrm{c}} q_{+}$ & ${ }^{\mathrm{c}} \sum \beta_{2}$ & $|q|$. & $=q_{-} \mid$ \\
\hline $\begin{array}{l}334 \\
\end{array}$ & 3-Pentanol & 0.330 & 0,363 & $\overline{0,381}$ & $\overline{0,56 \overline{0}}$ & 0,532 & 0,688 \\
\hline \multicolumn{8}{|c|}{ Hidrocarbonetos Alifáticos } \\
\hline s080 & Etino & 0,210 & 0,193 & 0,236 & 0,150 & 0,193 & 0,234 \\
\hline s081 & Propino & 0,120 & 0,196 & 0,333 & 0,170 & 0,193 & 0,607 \\
\hline s082 & 1-Butino & 0,120 & 0,195 & 0,320 & 0,150 & 0,190 & 0,559 \\
\hline s083 & 2-Butino & 0,000 & 0,046 & 0,237 & 0,150 & 0,184 & 0,569 \\
\hline s016 & Etano & 0.000 & 0,000 & 0,198 & 0,000 & 0,000 & 0,593 \\
\hline s017 & Propano & 0,000 & 0,000 & 0,201 & 0,000 & 0,000 & 0,568 \\
\hline \multicolumn{8}{|c|}{ Hidrocarbonetos Aromáticos e Derivados } \\
\hline s389 & Benzeno & 0.00 & 0,102 & 0,237 & 0.140 & 0,102 & 0,237 \\
\hline s564 & Nitrobenzeno & 0,00 & 0,134 & 0,303 & 0,280 & 0,534 & 0,404 \\
\hline \multicolumn{8}{|c|}{ Aminas e Nitrocompostos Alifáticos } \\
\hline s261 & Dimetilamina & 0,080 & 0,311 & 0,286 & 0,660 & 0,508 & 0,764 \\
\hline$s 265$ & Trimentilamina & 0,000 & 0,047 & 0,287 & 0,670 & 0,348 & 0,764 \\
\hline s284 & 1-Nitrobutano & 0.000 & 0,106 & 0,266 & 0,290 & 0,516 & 0,415 \\
\hline s286 & 1-Nitropentano & 0,000 & 0,106 & 0,266 & 0,290 & 0,516 & 0,415 \\
\hline \multicolumn{8}{|c|}{ Anilinas } \\
\hline s536 & Anilina & 0.260 & 0,300 & 0,311 & 0,410 & 0,571 & 0,851 \\
\hline$s 541$ & 2-Cloroanilina & 0,250 & 0,308 & 0,343 & 0,310 & 0,536 & 0,855 \\
\hline s542 & 3-Cloroanilina & 0,300 & 0,302 & 0,316 & 0,300 & 0,567 & 0,851 \\
\hline$s 543$ & 4-Cloroanilina & 0,300 & 0,301 & 0,315 & 0,310 & 0,566 & 0,851 \\
\hline s547 & 2-Nitroanilina & 0,300 & 0,367 & 0,384 & 0,360 & 0,577 & 0,907 \\
\hline$s 548$ & 3-Nitroanilina & 0,400 & 0,307 & 0,320 & 0,350 & 0,560 & 0,850 \\
\hline s549 & 4-Nitroanilina & 0,420 & 0,309 & 0,328 & 0,380 & 0,549 & 0,864 \\
\hline \multicolumn{8}{|c|}{ Ácidos Carboxilicos } \\
\hline s580 & Ácido Benzóico & 0,590 & 0,397 & 0,418 & 0,400 & 0,519 & 0,732 \\
\hline s582 & 3-Metíl Ácido Benzóico & 0,590 & 0,396 & 0,417 & 0,380 & 0,519 & 0,732 \\
\hline \multicolumn{8}{|c|}{ Fenóis } \\
\hline s589 & Fenol & 0,600 & 0,368 & 0,389 & 0,300 & 0,449 & 0,730 \\
\hline$s 621$ & 2-Fluor ofenol & 0,610 & 0,371 & 0,395 & 0,260 & 0,431 & 0,714 \\
\hline$s 622$ & 3-Fluorofenol & 0,680 & 0,371 & 0,394 & 0,170 & 0,445 & 0,726 \\
\hline$s 623$ & 4-Fluorofenol & 0,630 & 0370 & 0,391 & 0,230 & 0,446 & 0,728 \\
\hline$s 624$ & 2-Clorofenol & 0,320 & 0,371 & 0,397 & 0,310 & 0,437 & 0,713 \\
\hline$s 625$ & 3-Clorofenol & 0.690 & 0.370 & 0.394 & 0,150 & 0,446 & 0,726 \\
\hline s626 & 4-Clorofenol & 0,670 & 0,370 & 0,392 & 0,200 & 0,446 & 0,727 \\
\hline
\end{tabular}

${ }^{c}$ Os valores de $\Sigma \alpha_{2}$ e $\Sigma \beta_{2}$ foram compilados das referências ग’ . As cargas atômicas foram calculadas através do hamiltoniano $M N D O-P M 3$ usando o método $C M I .{ }^{\circ}$ cargas atômicas calculadas por método ab-intio usando funçóes de base 3-21G.

Analisando a Tabela 6.13, notamos que além da discrepância entre os valores dos parâmetros $\Sigma \alpha_{2}$ e $\Sigma \beta_{2}$ e os valores das cargas formais $q_{+}$e $\mid q$ - $\mid$ calculados por métodos semiempírico e ab-initio, os valores calculados para as cargas formais em ambos os métodos apresentam o mesmo comportamento. A discrepância encontrada deve-se ao fato de que as capacidades doadora e aceptora de pontes de hidrogênio diferem para solutos que sejam membros de uma série homóloga e/ou apresentem isomeria de posição. Por outro lado cargas formais calculadas, usando a definição de análise populacional de Mulliken para solutos homólogos e/ou isômeros de posição apresentam praticamente a mesma distribuição de carga. Isto deve-se à uma limitação intrínseca do cálculo da distribuição de carga no método de Mulliken, isto é, o método de Mulliken calcula apenas uma distribuição média de carga sobre cada átomo que compõem a molécula. 
Em resumo nossos resultados mostram que valores experimentais de $K_{\mathrm{s}}$ para a incorporação de solutos não iônicos em micelas formadas por detergentes aniônicos podem ser reproduzidos usando parâmetros teóricos como descritores. As equações de regressão linear teórica finais são consistentes com correspondentes equações de LSER baseadas em parâmetros experimentais. Porém quando o sistema em estudo é composto por micelas formadas por detergentes catiônicos ou não iônicos a metodologia apresenta limitações devido ao fato que os valores calculados para as cargas formais de solutos homólogos e/ou isômeros de posição, não poderam ser associadas a capacidade doadora e aceptora por pontes de hidrogênio. $\mathrm{Na}$ próxima seção discutiremos a interpretação físico-químico dos resultados apresentados nas seções 6.1.1 à 6.1.3, tomando como referência as equações de $L S E R$ para os mesmos conjunto de dados.

\subsection{Discussão e Interpretação Físico-Química dos Resultados}

Nas seções 6.1.1 à 6.1.3 mostramos os resultados da análise dos coeficientes de incorporação $\left(K_{s}\right)$ através de regressão linear múltipla usando parâmetros teóricos para descrever a incorporação de uma série de solutos não iônicos em micelas aniônicas, catiônicas e não iônicas. Mostramos que, para o mesmo conjunto de dados, as equações de regressão linear teóricas apresentam grau de correlação compatíveis com os das equações de LSER. Porém, uma boa correlação não é suficiente para determinar a validade da metodologia. Se os parâmetros teóricos escolhidos de fato espelham as propriedades específicas no ambiente de solubilização dos solutos, os valores e os sinais dos coeficientes da equação de regressão linear múltipla (teórica) devem ser compatíveis com aqueles das equações de $L S E R$, os quais foram mostrado por Quina et al. $^{2}$ e Leiva ${ }^{3}$ ser compatível com o processo de transferir um soluto e sua cavidade da fase aquosa até a fase micelar. Para este propósito, discutiremos a seguir, a interpretação físico-química das equações de regressão linear teórica tomando como base as equações de $L S E R$.

Primeiramente, observamos que, para os cinco detergentes estudados, os termos dominantes que contribuem para descrever a incorporação de solutos não iônicos em micelas de detergentes anionicos, catiônicos e não iônicos são o volume molar e a basicidade (capacidade aceptora de pontes de hidrogênio) do soluto. $O$ elevado valor positivo do coeficiente do termo $V_{x}$ reflete o fato de que é mais fácil criar uma cavidade na micela do que na água, devido à energia livre de coesão da água ser maior. Esta informação está contida nas equações (6.1) e (6.2) no caso das micelas formada por $S D S$, equações (6.9) e (6.12) no caso de micelas formadas por SDS-PEG, equações (6.15) e (6.18) no caso de micelas formadas por $D T A B$ e $C T A B$, respectivamente, e equações (6.23) e (6.26) no caso de micelas formadas por Brij-35.

O principal fator que favorece a partição para a fase aquosa é a basicidade do soluto, evidenciada pelo elevado valor negativo do coeficiente do termo $\Sigma \beta_{2}$ nas equações de $L S E R$. Este termo reflete o fato de que a água é melhor doador de pontes de hidrogênio do que os possíveis sítios de solubilização da micela. Em termos dos parâmetros teóricos, está informação esta contida nas equações (6.3), (6.11), (6.16), (6.20) e (6.25) para os casos de micelas formadas por $S D S, S D S-P E G, D T A B, C T A B$ e Brij-35 respectivamente. O significado físico-químico destas equações é que, para um próton ligado a um heteroátomo, como por exemplo um oxigênio hidroxílico ou fenólico, a proporcionalidade entre a basicidade do átomo de hidrogênio e a carga formal negativa do heteroátomo é modulada pela carga formal positiva do átomo de hidrogênio adjacente. 
As equações de LSER mostram que a solubilização micelar em geral é fracamente dependente da dipolaridade/polarizabilidade do soluto. A aparente insensibilidade de $K_{\mathrm{s}}$ em relação a dipolaridade é atribuído ao fato de que moléculas com valor significativo de dipolaridade serão solubilizadas próximo a interface micela-água, sugerindo assim uma similaridade nas dipolaridade da água e na região dos possiveis sítios de solubilização da micela. Esta interpretação é compatível com o fato de que os termos que descrevem a dipolaridade $\left(\pi_{\mathrm{F}}\right)$ e o momento de dipolo $<\mu>$ nas equaçōes de regressão linear teórica não ser estatisticamente significativo (veja Tabela 6.2 e 6.4 para os casos de incorporação em micelas formadas por $S D S$ e $S D S-P E G$, respectivamente) e por isso, não aparece nas equações de regressão final.

A fraca dependência da incorporação em relação a refração molar em excesso $\left(R_{2}\right)$ no caso de micelas formadas pelos detergentes SDS, DTAB e CTAB é associada ao fato que tanto a água quanto a micela formada por estes detergentes não geram um ambiente de solubilização polarizável. Porém, ao contrário, no caso das micelas formadas por agregados de detergente-polímero ( $S D S-P E G$ ) e Brij-35, a refração molar em excesso contribui de forma significativa para o processo de incorporação favorecendo a incorporação na fase micelar. Uma possível interpretação para este comportamento em termos de parâmetros teóricos está associada a troca de variável feita no estudo destes dois sistemas, isto é, podemos reescrever a equação (5.24) na forma abaixo, onde $R_{\mathrm{m}}$ representa a refração molar.

$$
\alpha(\lambda)=(3 / 4 \pi N) R_{m} 10^{24} \AA^{3}
$$

Analisando a equação (6.27), verifica-se que, o parâmetro teórico $\alpha_{\text {calc (0) }}$ contém contribuições devido a $R_{\mathrm{m}}$ observado diretamente através da equação (6.27) e devido ao volume molar descrito pelas relações (6.12) e (6.26). Desta forma a informação contida nas equações de regressão linear teórica para estes dois sistemas (quando a troca de parâmetro é levada em consideração) passa a ser consistente com o resultado descrito através das respectivas equações de $L S E R$. O fato do coeficiente de $R_{2}$ ser maior para a incorporação de solutos em SDS-PEG do que em SDS indica que o arranjo na interface agregado-água é bastante distinto daquela da micela de $S D S$, com participação simultânea de $S D S$ e de segmentos do $P E G$. A interpretação físico-química destas equações em termos da natureza do sítio de solubilização é que o meio que rodeia o soluto nas micelas de $S D S$ $P E G$ é significantemente menos hidrofóbico que o meio que rodeia o soluto nas micelas de $S D S$ puro. Assim, quando comparadas com micelas de $S D S$, o soluto incorporado nos agregados SDS-PEG deverá ter mais contato residual com água. Esta conclusão está de acordo com as observações experimentais de Zana et $\mathrm{al}^{7}$, mostrando que a relação entre as intensidades das bandas vibracionais $\left(\mathrm{I}_{1} / \mathrm{I}_{3}\right)$ do espectro de fluorescência do pireno é maior em agregados $S D S-P E G$ do que em micelas de $S D S$, indicando um ambiente mais polar. Já no caso do $B r i j-35$, a contribuição do termo $R_{2}$ é associado ao grupo polar polioxietileno do detergente Brij-35 que contribui para a polarizabilidade nos possíveis sítios de solubilização da micela.

A interpretação físico química para a contribuição do termo que descreve a acidez do soluto no processo de incorporação é a seguinte: No caso da incorporação de solutos não iônicos em micelas formadas por detergentes aniônicos, a acidez do soluto praticamente não contribui para a incorporação micelar, indicando que a basicidade das ligações por pontes de hidrogênio no ambiente de solubilização micelar é comparável à da água. Esta interpretação é consistente com fato de haver uma orientação preferencial das ligações O-H 
das moléculas de água na interface, sendo que os átomos de hidrogênio apontam na direção da fase aquosa. Por outro lado, a capacidade doadora de pontes de hidrogênio do soluto contribui para a incorporação de solutos não iônicos em micelas formadas por detergentes catiônico e não iônicos, favorecendo a incorporação na fase micelar. A distribuição de carga negativa na superfície das micelas formadas por $S D S$, e positiva nas micelas formadas por detergentes catiônicos, deve propiciar uma maneira de estabilizar as orientações das ligações $\mathrm{O}-\mathrm{H}$ das moléculas de água na interface de forma que os átomos de hidrogênio apontem na direção da fase aquosa. Além do mais nos detergentes catiônico as moléculas de água que rodeiam o contraíon devem ter capacidade aceptora de pontes de hidrogênio maior que na fase aquosa. Estes fatores devem aumentar a contribuição do termo $\Sigma \alpha_{2}$ para o caso de incorporação de solutos em micelas catiônicas, sendo maior no caso do $C T A B$, porque este possui um potencial de superfície maior que $D T A B^{\mathbf{8}, \mathbf{9}}$. No caso do Brij-35 a interpretação é consistente com aquela sugerida para o termo $R_{2}$, isto é, a capacidade aceptora de pontes de hidrogênio no sítio de solubilização da micela é melhorada pela presença dos átomos de oxigênio do grupo polioxietileno.

Nossos resultados mostram que valores experimentais de $K_{s}$ para incorporação de solutos não iônicos em micelas aquosas formadas por detergentes aniônico (micelas de $S D S$, dodecil sulfato de sódio e agregados de $S D S$ com $P E G$-polietilenoglicol) podem ser reproduzidos usando parâmetros teóricos. A relação linear resultante é quimicamente condizente com LSERS baseados nos parâmetros empíricos do soluto tabulados por Abraham.

No caso de detergentes catiônico e não iônico, a metodologia apresenta limitações quando usamos no conjunto de teste solutos homólogos e/ou que apresentem isômeria de posição por que, os métodos de calcular cargas formais usando a definição de análise populacional de Mulliken não podem ser associada ao caráter ácido e básico das pontes de hidrogênio. Alternativas para resolver a limitação imposta pelos parâmetros teóricos $q_{+} \mathrm{e}$ $|q-|$, porém mantendo a aproximação linear de energia livre são apontadas nos próximo capítulo.

\section{Referências}

1. Farah, J.P.S.; Lima. G.A.R.; Quina, F.H., 1997, J. Mol. Struct.(THEOCHEM),394,167.

2. Quina, F.H.; Alonso, E. O.; Farah, J.P.S.; 1995, J. Phys. Chem.; 99, 11708.

3. Leiva, O.W.; 1996, Dissertação de Doutorado, Instituto de Química, Universidade de São Paulo, Brasil.

4. Abraham, M.H.; Chadham, H.S.; Whiting, G.S.; Michell, R.C., 1994, J. Pharm. Sci., $\mathbf{8 3}, 1085$.

5. Abraham, M.H.; 1993, Chem. Soc. Rev.,22, 73.

6. Rodrigues, M.A.; Alonso, E.O.; Yihwa, C.; Farah, J.P.S.; Quina, F.H.,1999, Langmuir, 15, 6770 .

7. Zana, R.; Lang, J.; Lianos, P.;1982, Polym. Prep. ACS.,23(1),39.

8. Zana, R.; 1980, J. Colloid Interface Sci.,78, 330.

9. Buckingham, S.A.; Garvey, C.J.; Warr, G.G.; 1993, J. Phys. Chem., 97, 102 


\section{Eapítulo 7}

\section{Conclusão}

Os resultados apresentados neste estudo, confirmam nossa expectativa em substituir os parâmetros empíricos do soluto tabulados por Abraham, por parâmetros teóricos obtidos a partir da estrutura molecular do soluto.

No caso de detergentes catiônicos e não iônicos a metodologia apresenta limitações quanto ao emprego de solutos homólogos e/ou isômeros de posição, devido as cargas formais calculadas para estes solutos não poderem ser associadas a capacidade doadora e aceptora por pontes de hidrogêneo. 


\subsection{Conclusão}

Os resuitados apresentados e discutidos neste trabalho mostram que, valores experimentais de $K_{s}$ para incorporação de solutos não iônicos em micelas formadas por detergentes aniônicos, podem ser reproduzidos, usando parâmetros teóricos como descritores. As equações de regressão linear resultantes permitem estimar coeficientes de incorporação micelar com precisão de um fator de dois, se conhecermos a estrutura molecular do soluto. A interpretação físico-química das equações de regressão linear teórica, são consistentes com as equações de LSER (baseadas em parâmetros experimentais) obtidas por Quina et. al. e Leiva para o mesmo conjunto de dados. Além do mais, o fato do termo $\Sigma \beta_{2}$ das equações de $L S E R$ poder ser descrito através das cargas formais $\left(\Sigma \beta_{2}=\right.$ cte $\left.+\left|q_{-}\right|-q_{+}\right)$, adiciona uma informação importante ao entendimento dos fatores que contribuem para a incorporação. Ou seja, para um próton ligado a um heteroátomo como por exemplo, o oxigênio hidroxílico ou fenólico, a proporcionalidade entre a basicidade do átomo de hidrogênio e a carga formal negativa do heteroátomo é modulada pela carga formal positiva do átomo de hidrogênio adjacente ${ }^{1}$.

Os resultados confirmam nossa expectativa de substituir os parâmetros empíricos tabulados por Abraham por parâmetros teóricos obtidos unicamente a partir da estrutura molecular do soluto.

No caso de detergentes catiônicos e não iônicos a metodologia apresenta limitações quanto ao emprego de solutos homólogos e/ou isômeros de posição, devido as cargas formais calculadas (a partir da definição de análise populacional de Mulliken) para estes solutos não poderem ser associadas a capacidade doadora e aceptora por pontes de hidrogênio. Uma alternativa para expandir o uso da metodologia para micelas formadas por detergentes catiônicos e não iônicos mantendo o enfoque de Famini e incluindo solutos homólogos e/ou isômeros de posição é, procurar outras maneiras de calcular cargas formais que possam ser associadas a capacidade doadora e aceptora de pontes de hidrogênio. Neste sentido realizamos estudos preliminares enfocando a descrição da ligação química através do uso direto da distribuição da densidade de carga e relacionando esta quantidade as forças atuantes entre os núcleos através do teorema de Hellmann-Feynman, ou seja, a distribuição total de carga é analisada em termos da quantidade de carga obtida em diferentes regiões do espaço que envolve a molécula, em outras palavras uma análise topológica da distribuição de carga ${ }^{2}$.

Uma abordagem alternativa é tentar correlacionar propriedades estruturais e/ou eletrônicas com os parâmetros empíricos do soluto, e desse modo obter uma fórmula ou receita para estimá-los a partir de cálculos de estrutura eletrônica. Neste sentido realizamos exaustivas análises de regressão linear envolvendo uma série de parâmetro extraídos de cálculos de química quântica. Os resultados obtidos para o conjunto teste de 672 solutos mostram que, podemos usar a equação de Clausius-Mossotti para obter o parâmetro $R_{2}$ a partir do cálculo da palarizabilidade molecular e volume molar $\left(R_{2}=0,30\right.$ - 3,06 $\left.\mathrm{V}_{\mathrm{x}} / 100+2,47 \alpha_{\text {calc (0) }} / 10 ; R=0,963, s d=0,15, F=4329,5\right)$. Para os parâmetros empíricos $\Sigma \alpha_{2}$ e $\Sigma \beta_{2}$ respectivamente obtivemos as seguintes equações de regressão ( $\Sigma \alpha_{2}$ $\left.=-0,10+1,48 q_{+} ; R=0,883 ; s d=0,10 ; F=2435,3\right)$ e $\left(\Sigma \beta_{2}=0,06+0,87\left|q_{-}\right| ; R=0,81 ;\right.$ $s d=0,12 ; F=1300$ ). Recentemente Abraham et. al. ${ }^{3}$ realizaram estudos envolvendo equivalente de grupo (usando 31 fragmentos como descritores) para tentar reproduzir os 
parâmetros empíricos do soluto por parâmetros calculados a partir da estrutura molecular. Nossos resultados apresentam grau de correlação na mesma ordem de grandeza daqueles obtidos por Abraham. Porém nossa metodologia necessita de dois parâmetros teóricos para correlacionar com $R_{2}$ e, um parâmetros para correlacionar com $\Sigma \alpha_{2}$ e $\Sigma \beta_{2}$.

Nossos resultados também mostram que os parâmetros $\varepsilon_{H O M O}$, e $\varepsilon_{L U M O}$, escolhidos para refletir possíveis contribuições covalentes na formação de pontes de hidrogênio e o momento de dipolo $\langle\mu\rangle$ escolhido para descrever as interações do tipo dipolo-dipolo e dipolo-dipolo induzido da fase com o soluto não exercem influencia nos fatores envolvidos na incorporação micelar. Observamos ainda que, o parâmetro teórico $\pi_{\mathrm{F}}$ sugerido por Famini para refletir as interações de dipolaridade/polarizabilidade não tem relação com o parâmetro empírico de dipolaridade/polarizabilidade $\pi_{2}$ e que o nível de cálculo empregado não é relevante para obter o valor dos parâmetros teóricos.

Em resumo, este estudo mostra pela primeira vez, uma análise detalhada do uso de relação linear teórica de energia livre de solvatação para estudar incorporação de solutos não iônicos em micelas aquosas formadas por detergentes aniônicos, catiônicos e não iônicos visando elucidar e esclarecer os fatores que influenciam na solubilização ou incorporação de solutos nestas micelas.

\section{Referências}

1. Farah, J.P.S.; Lima, G.A.R.; Quina, F.H.; 1997, J. Mol. Struct. (THEOCHEM),394,267.

2. Bader, R.F.W.; 1990, Atom in Molecules: A quantum theory, Claredon Press, Oxford.

3. Platts, J.A.; Butina, D.; Abraham, M.H.; Hersey, A.;1999, J. Chem. Inf. Comput. Sci, 39, 835 . 


\section{OBSERVAÇÃO}

\section{NÃO FOI AUTORIZADA A INCLUSÃO DO TRABALHO NESTE ARQUIVO}

